\title{
PHYSIK-DEPARTMENT
}

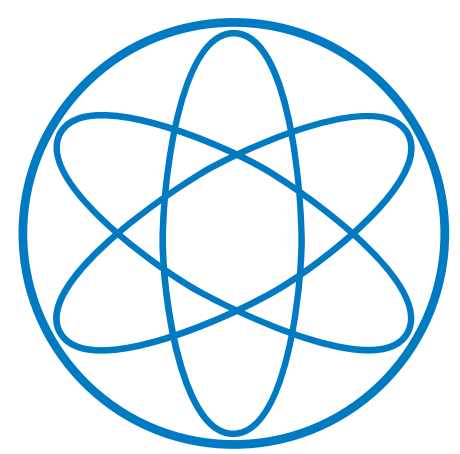

Spin-Polarized

Scanning Tunneling Microscopy

at the Solid/Liquid Interface

Dissertation von

Claudia Majer

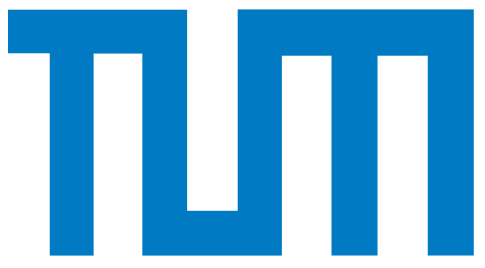

Technische Universität München 



\title{
TECHNISCHE UNIVERSITÄT MÜNCHEN
}

\author{
Lehrstuhl E20
}

Molekulare Nanowissenschaften \& Chemische Physik von Grenzflächen

\section{Spin-Polarized \\ Scanning Tunneling Microscopy at the Solid/Liquid Interface}

Sybille Claudia Majer

Vollständiger Abdruck der von der Fakultät für Physik der Technischen Universität München zur Erlangung des akademischen Grades eines Doktors der Naturwissenschaften (Dr. rer. nat.) genehmigten Dissertation.

Vorsitzender: Univ.-Prof. Dr. Martin Zacharias

Prüfer: $\quad$ 1. Priv.-Doz. Dr. Werner Schindler

2. Univ.-Prof. Dr. Katharina Krischer

Die Dissertation wurde am 21.03.2013 bei der Technischen Universität eingereicht und durch die Fakultät für Physik am 05.06.2013 angenommen. 


\begin{abstract}
The spin-polarized scanning tunneling microscopy (sp-STM) is demonstrated between a magnetized STM tip and a magnetic substrate at the solid/liquid interface under electrochemical conditions at room temperature. Thereby, sp-STM at the solid/liquid interface is accomplished using the differential magnetic mode established by Wulfhekel and Kirschner for investigations at the solid/vacuum interface. The implementation of the sp-STM setup into the electrochemical STM is an important part of this thesis. Special attention is paid to the magnetostriction effect of possible magnetic STM tips. As a model system extended Co islands are electrochemically grown on an $\mathrm{Au}(111)$ single crystal. An appropriate electrochemical environment is required to ensure metal Co islands on $\mathrm{Au}(111)$, since Co deposition occurs at low potentials where hydrogen evolution and dissolved oxygen contributes to electrochemical reactions at the substrate. After sample preparation extended Co islands on $\mathrm{Au}(111)$ up to four atomic layers in height are observed with bare $\mathrm{Au}(111)$ areas in between, which is an ideal sample surface for sp-STM at the solid/liquid interface showing ferromagnetic and diamagnetic areas. Sp-STM is employed in-situ at the solid/liquid interface to observe not only sample topography but also sample magnetization of the model system $\mathrm{Co} / \mathrm{Au}(111)$ with high spatial resolution.
\end{abstract}




\section{Zusammenfassung}

Die vorliegende Arbeit beschäftigt sich mit Spin-polarisierter RasterTunnel-Mikroskopie (engl. Abk. sp-STM) an einer magnetischen Probe mittels einer magnetischen Spitze im elektrochemischen Umfeld bei Raumtemperatur. Für die Realizierung wird dabei sp-STM im differential magnetic Modus angewendet, der durch Wulfhekel and Kirschner für Untersuchungen an Proben im Vakuum etabliert wurde. Die Implementierung eines sp-STM Aufbaus in den bestehenden Aufbau eines STM für Beobachtungen an der fest/flüssig Grensfläche ist ein essenzieller Bestandteil dieser Arbeit. Besonderes Augenmerk wird dem Magnetostriktionseffekt potentieller magnetischer STM-Spitzen beigemessen. Als Modelsystem für sp-STM werden ausgedehnte Co Inseln auf einem $\mathrm{Au}(111)$ Einkristall elektrochemisch abgeschieden. Parallel zur Co Abscheidung treten ebenso Wassersoffentwicklung und elektrochemische Reaktionen mit gelöstem Sauerstoff am Goldsubstrat auf, da sie ebenfalls bei diesen Potentialen stattfindet. Um Weiterreaktionen des abgeschiedenen Co zu verhindern, ist eine geeignete elektrochemische Umgebung notwendig. Die resultierenden ausgedehnten Co Inseln sind bis zu vier Atomlagen hoch und separiert durch weite Bereiche unbedeckter Goldoberfläche. Dadurch wird eine ideale Probenoberfläche für sp-STM im elektrochemischen Umfeld prepariert, die ferromagnetische und diamagentische Bereiche aufweist. Die sp-STM Untersuchungen können nun direkt an der fest/flüssig Grenzfläche erfolgen, wodurch es ermöglicht wird sowohl die Topografie als auch die Magnetisierung der Probenoberfläche örtlich hoch aufgelöst in einer Messung zu untersuchen. 
to my family

Sybille Klaus Ariane Tjark 


\section{Contents}

$\begin{array}{lll}\text { Abstract } & \text { III] }\end{array}$

List of Figures

List of Tables

1 Introduction 1

2 Electrochemistry

2.1 Electrochemistry - Theoretical Approach . . . . . . . . . . 6

2.1.1 Metal Deposition . . . . . . . . . . . . . 6 6

2.1 .2 The Solid/Liquid Interface . . . . . . . . . . . . . 8

2.2 Electrochemical Setup . . . . . . . . . . . . . . . . . . . . 10

2.3 Characterization ................... 11

2.3.1 Cyclic Voltammetry . . . . . . . . . . . . . 12

2.3.2 Deaeration of the Electrolyte . . . . . . . . . . . . . 13

2.3.3 Cleanliness of the Electrochemical Environment . . . . . . . 15

2.4 Deposition of $\mathrm{Co}$ on $\mathrm{Au}(111) \ldots \ldots \ldots \ldots$. . . . . . . . . . . . . . . . . . . . .

2.4.1 Shift of pH-value . . . . . . . . . . . . . 17

2.4.2 Resulting Co Deposition on $\mathrm{Au}(111)$. . . . . . . . . . . 22

2.5 Conclusion . . . . . . . . . . . . . . . . 23

3 Scanning Tunneling Microscopy 25

3.1 STM - Theoretical Approach . . . . . . . . . . . . . . . . 26

3.2 STM in the Electrochemical Environment . . . . . . . . . . . 28

3.2.1 Setup of the Ec-STM . . . . . . . . . . . . . . . 29

3.2.2 Ec-STM Tips - Fabrication and Characterization . . . . . . . 31

3.2.3 STM of $\mathrm{Au}(111)$ at the Solid/Liquid Interface . . . . . . . . . 33

$3.3 \mathrm{STM}$ of $\mathrm{Co} / \mathrm{Au}(111)$ at the Solid/Liquid Interface . . . . . . . . . . 34

3.3 .1 Ec-STM Investigations . . . . . . . . . . . . . . . . 35

3.3.2 Morphology of $\mathrm{Co}(0001)$ on $\mathrm{Au}(111) \ldots \ldots . . . \ldots 38$ 
3.3.3 Formation of Co-Au Alloy . . . . . . . . . . . . . . . . . 39

3.4 Conclusion . . . . . . . . . . . . . . . . . . . 42

4 Spin-polarized Scanning Tunneling Microscopy 45

4.1 Sp-STM - Theoretical Approach . . . . . . . . . . . . . . 46 46

$4.2 \quad$ Sp-STM Implementation . . . . . . . . . . . . . . . . . . . . . . 48

4.2.1 Modes of Operation . . . . . . . . . . . . . . . . . . 49

4.2 .2 Sp-STM Integration . . . . . . . . . . . . . 51

4.3 Magnetic Tips . . . . . . . . . . . . . . . . . . 55

4.3.1 Magnetostriction ................ 5 54

4.3.2 STM Setups to Observe Magnetostriction . . . . . . . . . . 58

4.3.3 Investigation of Magnetostriction . . . . . . . . . . . 62

4.3.4 Magnetostriction at High Modulation Frequency . . . . . . . . 69

4.4 Sp-STM on $\mathrm{Co} / \mathrm{Au}(111)$ at the Solid/Liquid Interface . . . . . . . . . 71

4.4.1 Magnetic Behavior of $\mathrm{Co} / \mathrm{Au}(111) \ldots . . . . . . . . .771$

4.4 Experiment . . . . . . . . . . . . . . . . 72

4.5 Effects at the Second Harmonic . . . . . . . . . . . . . . . 78

4.5.1 Magnetostriction . . . . . . . . . . . . . . . . 797

4.5.2 Magnetic Susceptibility . . . . . . . . . . . . . . . . 80

4.5.3 Experiment .......................... 83

4.6 Conclusion . . . . . . . . . . . . . . . . . . 85

5 Summary \& Outlook 89

A Lists of Chemicals, Materials and Devices 93

B Abbreviations

List of Publication

References

Acknowledgments 113 


\section{List of Figures}

1.1 Thematic triangle . . . . . . . . . . . . . . . . 3

2.1 Metal deposition/dissolution . . . . . . . . . . . . . 6

2.2 Double-layer model . . . . . . . . . . . . . . . . . . . . . . . . . 9

2.3 Double-layer potential profile . . . . . . . . . . . . . . . 9

2.4 Schematic diagram of the electrochemical cell . . . . . . . . . 10

2.5 Example for cyclic voltammetry . . . . . . . . . . . . . 12

2.6 Deaeration of the supporting electrolyte investigated by CV . . . 14

2.7 Cleanliness investigated by $\mathrm{CV} \ldots \ldots \ldots \ldots \ldots$

2.8 Cleanliness investigated by CV, Tafel plot $\ldots \ldots \ldots \ldots \ldots$

2.9 Pourbaix diagram for the $\mathrm{Co} / \mathrm{H}_{2} \mathrm{O}$ system $\ldots \ldots \ldots \ldots \ldots$

2.10 Investigation of the diffusion coefficient $D_{H^{+}} \ldots \ldots \ldots \ldots$

2.11 Calculated concentration profile . . . . . . . . . . . 20

$2.12 \mathrm{CVs}$ of Co deposition/dissolution changed by shifting $\mathrm{pH}$-value at WE 21

$2.13 \mathrm{CVs}$ without/with $\mathrm{CoSO}_{4}$ in solution . . . . . . . . . . 23

3.1 Modes of scanning tunneling microscopy $\ldots \ldots \ldots \ldots$

3.2 Setup of the electrochemical STM . . . . . . . . . . . 29

3.3 Tip preparation . . . . . . . . . . . . . . . . . . . . . . . 31

3.4 Sketched etching current vs. time . . . . . . . . . . . 32

3.5 SEM images of an etched Au tip . . . . . . . . . . . . . . 32

3.6 Topography of $\mathrm{Au}(111)$ herringbone reconstructions . . . . . . . 34

3.7 Topography of deposited Co with moiré structure on top I . . . . . 36

3.8 Topography of deposited Co with moiré structure on top II . . . . . . 37

3.9 STM and CV of Co dissolution . . . . . . . . . . . . 40

3.10 1. CV and 2. CV of $\mathrm{Au}(111)$ with $\mathrm{CoSO}_{4}$ in solution . . . . . . 41

4.1 Cosine dependence of the tunneling conductance . . . . . . . . . 46

4.2 Schematic illustration of the anti-/parallel tunneling . . . . . . 47

4.3 Tunneling current in anti-/parallel orientation . . . . . . . . . 48

4.4 Principle of sp-STM . . . . . . . . . . . . . . . 50

4.5 Coil of the sp-STM setup . . . . . . . . . . . . . 52 
4.6 Setup of the sp-STM . . . . . . . . . . . . . . 53

4.7 Illustration of the magnetostriction effect . . . . . . . . . . . 55

4.8 Saturated magnetostriction . . . . . . . . . . . . . 56 56

4.9 Non-saturated magnetostriction . . . . . . . . . . . . . . . 57

4.10 STM setups for investigation on magnetostriction . . . . . . . . . 58

4.11 Sketch of magnetostriction measurement . . . . . . . . . . . . . 59

4.12 Magnetic field strength of the coils . . . . . . . . . . . . . . . . 60

4.13 The magnetic field of the coil . . . . . . . . . . . . . . . 61

4.14 SQUID measurements . . . . . . . . . . . . . . . 6 . 63

4.15 Magnetostriction measurement of AC20 . . . . . . . . . 65

4.16 Magnetostriction measurements of AC20 with increasing magnetic

field strength . . . . . . . . . . . . . . 66 66

4.17 Magnetostriction measurements of $\mathrm{NiAu} \ldots \ldots . \ldots 6$

4.18 Magnetostriction measurements of $\mathrm{Ni} \ldots \ldots$. . . . . . . . . . . . . . . . .

4.19 Magnetostriction at high modulation frequency, AC20 . . . . . . . . 70

4.20 Magnetization of Co bulk and thin Co layers . . . . . . . . . . . 72

$4.21 \mathrm{Sp}-\mathrm{STM}$ of $\mathrm{Co} / \mathrm{Au}(111) \ldots \ldots \ldots \ldots$

4.22 Large scale sp-STM of $\mathrm{Co} / \mathrm{Au}(111)$, topography . . . . . . . . . . 75

4.23 Large scale sp-STM of $\mathrm{Co} / \mathrm{Au}(111), \Delta I_{s p}^{f_{\text {mod }}} \ldots \ldots \ldots$. . . . . . . . . . . . . . .

$4.24 \mathrm{Sp}-\mathrm{STM}$ control experiment . . . . . . . . . . . . 77

4.25 Influence of magnetostriction on sp-STM . . . . . . . . . . . 79

4.26 Magnetic susceptibility . . . . . . . . . . . . . . . . 81

4.27 Illustrated origin of $I_{s p}^{2 f_{\mathrm{mod}}} \ldots \ldots \ldots \ldots \ldots \ldots$

4.28 Principle of $\mathrm{sp}-\mathrm{STM}$ at $2 f_{\bmod } \ldots \ldots \ldots \ldots$

$4.29 \mathrm{Sp}-\mathrm{STM}$ of $\mathrm{Co} / \mathrm{Au}(111)$ at $\left(2 f_{\mathrm{mod}}\right)$, topography . . . . . . . . 84

$4.30 \mathrm{Sp}-\mathrm{STM}$ of $\mathrm{Co} / \mathrm{Au}(111)$ at $\left(2 f_{\mathrm{mod}}\right), \Delta I_{s p}^{2 f_{\text {mod }}} \ldots \ldots \ldots$ 


\section{List of Tables}

2.1 Standard potentials . . . . . . . . . . . . . 7

4.1 Saturation magnetostriction coefficients, single crystal . . . . . . . . 54

4.2 Saturation magnetostriction coefficients, polycrystalline materials . . 55

4.3 SQUID measurements . . . . . . . . . . . . . . . . 64 64

A.1 List of chemicals . . . . . . . . . . . . . . . . . 93

A.2 List of materials . . . . . . . . . . . . . . . . . . . 94

A.3 List of devices . . . . . . . . . . . . . . . . . . . . . 94 


\section{Chapter 1}

\section{Introduction}

Magnetism has fascinated humans since magnetic stones have become known, first described by Thales of Miletus (about 634-546 BC) [185]. One of the first applications of such magnetic materials were magnetic compasses used for navigation. In this way, magnetism has begun to influence our history because of new technological possibilities [185]. The phenomenon of magnetism was explored by great scientists such as Gauss, Oersted, Faraday, Maxwell, Bohr and Heisenberg, to name a few [24, 54, 61, 81, 123, 136]. Nowadays more than ever, advanced magnetic applications are present in our daily life, particularly with regard to magnetic data storage.

Especially concerning the cost-efficient manufacture of magnetic devices, electrochemistry offers the opportunity of low-cost and fast mass production. Therefore, magnetic material can be electrochemically deposited out of an electrolyte solution by potential shifts, and thus, ultrathin magnetic films can be fabricated [109, 162, 163]. The layer of the deposited material can vary considerably in its topology in dependence of parameters such as temperature, the actual deposition potential, the total deposition time and the kind of the solid surface where to deposit on. The resulting magnetic properties are strongly correlated to the growth process of the layer [28, 30, 97]. Therefore, a possibility to investigate magnetic properties of the deposited material in-situ at the solid/liquid interface is highly appreciated.

Available in-situ techniques such as magneto-optical Kerr effect measurements [2, 146, 161, 162] or alternating gradient field magnetometry [5, 33, 73] are methods integrating over a certain surface area. These methods are not sufficient enough to enable highly localized investigations of the magnetization depending on the topology.

For ex-situ investigations on the magnetic properties out of the electrochemical environment, a protecting layer on top is utilized, electrochemically deposited in a second step. This additional interface between the magnetic layer and protective layer influences the observed magnetic properties [28, 34, 38, 55, 184], and therefore, 


\section{INTRODUCTION}

the magnetic properties caused by the system magnetic layer/substrate itself can not be investigated directly.

A powerful tool to investigate surfaces is given by scanning probe microscopy. Thereby, the surface of a solid sample can be probed down to atomic resolution by means of a small tip. Investigations by atomic force microscopy (AFM) and scanning tunneling microscopy (STM) are of particular relevance and widely applied. STM was first realized by Binnig and Rohrer in 1981 [15, 16, 17, 18, 19] and in 1986 this pioneering development was honored with the Nobel Prize, shared with Ruska for the development of the electron microscope [154, 155, 156]. With modified STMs not only the topology of a surface can be investigated, but also the magnetism of magnetic samples [23, 199, 203]. Since this effect is caused by the spin-polarization, this kind of investigation is called spin-polarized scanning tunneling microscopy (spSTM).

STM was not only accomplished in vacuum or air, but also at the solid/liquid interface [63]. On the one hand, this offers the opportunity to investigate processes at this interface by electrochemical methods [62, 179]. On the to other hand, the resulting surface can be probed additionally by STM in-situ [51, 88, 108, 120. Furthermore, STM at the solid/liquid interface can be used to fabricate nanostructures by electrochemical deposition induced by the electric field of the tip [96] or potential pulsing of the tip near the surface [164, 165, 166, 167].

As a logical consequence of STM advanced at the solid/liquid interface and the sp-STM, a combination of both techniques, the sp-STM at the solid/liquid interface, would be a suitable tool to investigate the magnetic properties of a magnetic surface in an electrochemical environment in-situ.

The subject of this thesis is a combination of the three topics introduced above: Electrochemistry, scanning probe microscopy and investigations on magnetic properties. This thematic triangle is illustrated in Figure 1.1 by three overlapping circles. Thereby, the overlaps symbolize combinations of these topics and the arrow reflects the thematic structure of the thesis. The overlap of all three topics is the central topic of the thesis: investigations on sample magnetization at the solid/liquid interface by spin-polarized scanning tunneling microscopy. This work is structured straight on in accordance to the topic interaction. Each chapter consists of a theoretical approach, a detailed description of the setup in use, resulting measurements and their interpretation, ending with conclusions.

As a model system for sp-STM at the solid/liquid interface, ferromagnetic Co is electrochemically deposited on a non-ferromagnetic $\mathrm{Au}(111)$ substrate.

Starting from electrochemistry, the electrochemical setup and further sample preparation are discussed in Chapter 2. Initially, electrochemical investigations on the $\mathrm{Au}(111)$ single crystal in use demonstrate the deaeration of the electrolyte and cleanliness of the electrochemical environment. 


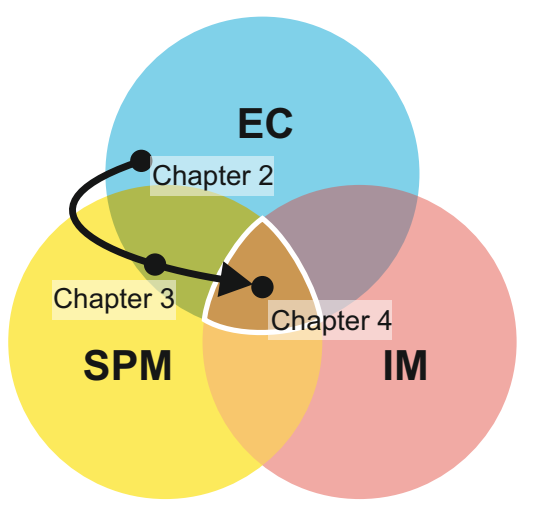

Figure 1.1: Thematic triangle of this thesis: blue circle - electrochemistry (EC), yellow circle - scanning probe microscopy (SPM), and red circle - investigations on the magnetization (IM). The overlaps symbolize combined methods - the center represents sp-STM at the solid/liquid interface. The arrow reflects the structure of the thesis.

Further, the model system $\mathrm{Co} / \mathrm{Au}(111)$ is electrochemically prepared and investigated by electrochemical methods. Co deposition and its remaining on the $\mathrm{Au}(111)$ surface occurs in a potential range where hydrogen evolution arises as well. With regard to ensure a metal $\mathrm{Co} / \mathrm{Au}(111)$ surface for further investigations by STM, the influence of this parasitic reaction on the sample preparation is discussed additionally.

The STM investigations on $\mathrm{Co} / \mathrm{Au}(111)$ at the solid/liquid interface are presented in Chapter 3. In the beginning, the setup in use is introduced as well as the preparation of the STM tips. The performance of the electrochemical STM (ec-STM) is demonstrated by observation of the $\sqrt{3} \times 22$ reconstructed $\mathrm{Au}(111)$ surface.

After the Co deposition, the topology of the model system is investigated by ecSTM. Furthermore, the ec-STM setup offers the possibility for monitoring Co deposition/dissolution in-situ. Thereby, the formation of a Co-Au alloy at the $\mathrm{Au}(111)$ surface is observed independently by ec-STM and electrochemical methods.

The implementation of a sp-STM setup is discussed in Chapter 4. Sp-STM at the solid/liquid interface is realized by use of the differential magnetic mode, which was established by Wulfhekel and Kirschner for applications inside an UHV apparatus 202. Initially, the magnetic properties of various magnetic tip materials are investigated by SQUID. Especially the magnetostriction effect is supplementary investigated by adapted STM setups. Finally, it is demonstrated that sp-STM is accomplished at the solid/liquid interface. Using the model system $\mathrm{Co} / \mathrm{Au}(111)$, this technique allows to map out-of plane magnetization. Additional effects caused by the sp-STM setup are named and discussed at the end.

The last Chapter 5 summarizes the presented results and addresses open questions as well as future perspectives. 
1. INTRODUCTION 


\section{Chapter 2}

\section{Electrochemistry}

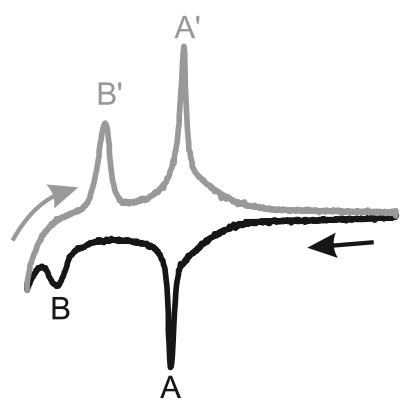

The scanning tunneling microscopy (STM) experiments that are subsequently presented in this thesis were performed in an electrochemical environment at room temperature. An interface between liquid solvent and solid substrate is investigated, thus constituting a liquid-solid interface situated in an electrochemical cell [115, 177, 196]. By ensuring very clean experimental conditions during measurement, the tunneling conditions at the solid/liquid interface are similar to those formed under ultra-high vacuum (UHV) conditions [160, 161, 162]. In addition, electrochemistry offers an elegant possibility of both metal deposition and dissolution on a substrate without any heating or sputtering steps.

In Section 2.1 some general theoretical aspects of electrochemistry such as metal deposition/dissolution and the solid/liquid interface are briefly treated. The electrochemical setup in use is detailed in Section 2.2. Thereby, the deaeration as well as the purity of the electrolyte solution are monitored by use of cyclic voltammetry [62], which is an important tool in electrochemistry. The sample preparation of electrochemically deposited $\mathrm{Co}$ on $\mathrm{Au}(111)$ is discussed in Section 2.4. Since Co deposition takes place at low potentials where hydrogen evolution arises, an increasing $\mathrm{pH}$-value precedes the formation of cobalt hydroxide complexes. Corresponding electrochemical investigations are shown, and further, the supporting electrolyte is modified to avoid cobalt hydroxide complexes. 


\section{ELECTROCHEMISTRY}

\subsection{Electrochemistry - Theoretical Approach}

An electrochemical cell consists of a substrate acting as the working electrode (WE), a reference electrode (RE), and a counter electrode (CE), all placed within a supporting electrolyte. Thereby, the potential of the working electrode $E_{\mathrm{WE}}$ is adjusted with respect to the reference electrode ${ }^{1}$.

For investigations of deposited metal on the substrate, a solution of interest is added to the electrolyte such as solvated metal sulfates $M_{(a q)}^{z+}$ and $\mathrm{SO}_{4}^{2-}(a q)$. Since the supporting electrolyte should conform to the admixture of interest, for metal sulfates the electrolyte should be an aqueous solution of sulfuric acid $\left(\mathrm{H}_{2} \mathrm{SO}_{4}\right)$ or sodium sulfate $\left(\mathrm{Na}_{2} \mathrm{SO}_{4}\right)$.

Metal deposition out of this electrochemical solution is the subject of discussion in the first section. Following, a short approach of the solid/liquid interface is given. A more detailed introduction to electrochemistry can be found in numerous books and publications, for example [78, 171].

\subsubsection{Metal Deposition}

The reduction reaction of solvated metal cations $M_{(a q)}^{z+}$ at the working electrode occurs for a potential of the working electrode $E_{\mathrm{WE}}$ more negative with respect to a certain red/ox potential $E\left(M e / M^{z+}\right)$. Thereby, the metal cations $M^{z+}$ in solution adsorb onto the surface of the working electrode and discharge, as illustrated in Figure 2.1. Following adsorption, the adatoms diffuse on the surface and preferentially attach to defects, step edges or engage in cluster formation. The reversed process, the oxidation reaction of the deposited metal $M$, occurs subsequently at a potential more positive than $E\left(M e / M^{z+}\right)$,

$$
M^{z+}+z e^{-} \rightleftarrows M
$$

Figure 2.1: Deposition of solvated cations (1) on a metal surface and the reverse dissolution (2). Adatoms can diffuse on the surface until they are attached (3) to a defect such as step edges or already existing deposited metal clusters.

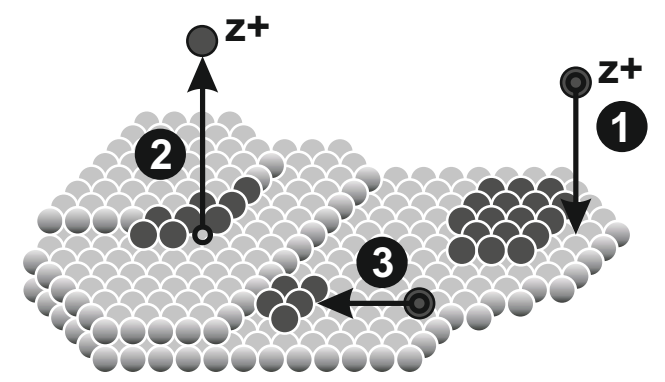

\footnotetext{
${ }^{1}$ The electrochemical red/ox reaction at the reference electrode defines the zero potential of the electrochemical setup by its standard potential $E_{00}(\mathrm{red} / \mathrm{ox})$.
} 
Deposition and dissolution of each red/ox couple $M / M^{z+}$ occurs at the respective standard potential $E_{0}\left(M / M^{z+}\right)$, defined for a concentration $c=1 \mathrm{moll}^{-1}=1 \mathrm{M}$ in the aqueous solution.

The correlation between any concentration $c_{o x}$ in solution and the deposition/dissolution potential for each red/ox reaction is described by the Nernst equation of the electrode potential [134]

$$
E_{0}(\text { red } / \text { ox })=E_{00}(\text { red } / \text { ox })+\frac{R T}{z F} \cdot \ln \left(\frac{a_{o x}}{a_{\text {red }}}\right)
$$

depending on the standard potential of the red/ox couple $E_{00}(r e d / o x)$ and the activity of each of the reactants $a_{o x}$ and $a_{\text {red }}$, where $a_{i}=c_{i} \cdot \gamma_{i}$ depends on the concentration $c_{i}$ of reactant $i=o x$, red and its activity coefficient $\gamma_{i}$. Since the activity $a_{M}$ of the pure metal is set to be 1 and the activity coefficient of a highly diluted solution $\gamma_{i} \approx 1$, the Nernst equation for $M / M^{z+}$ can be written as

$$
E_{0}\left(M / M^{z+}\right)=E_{00}\left(M / M^{z+}\right)+\frac{0.0256 \mathrm{~V}}{z} \cdot \ln \left(c_{M^{z+}}\right),
$$

for $T=298 \mathrm{~K}, R=8.32 \mathrm{~J} \mathrm{~K}^{-1} \mathrm{~mol}^{-1}, F=96485.34 \mathrm{C} \mathrm{mol}^{-1}$ and $\frac{a_{M^{z+}}}{a_{M}}=c_{M^{z+}}$.

In Table 2.1 standard potentials $E_{00}$ for some red/ox reactions are exemplarily listed. For historical reasons all standard potentials are quoted with respect to the

\begin{tabular}{|c|c|c|}
\hline red/ox couple & & $E_{00}$ vs SHE \\
\hline $\mathrm{O}_{2}+4 \mathrm{H}^{+} / 2 \mathrm{H}_{2} \mathrm{O}$ & & $1.229 \mathrm{~V}$ \\
\hline $\mathrm{Cu}^{2+} / \mathrm{Cu}$ & & $0.342 \mathrm{~V}$ \\
\hline $\mathrm{Hg}_{2} \mathrm{Cl}_{2} / 2 \mathrm{Hg}+2 \mathrm{Cl}^{-}$ & $(\mathrm{SCE})$ & $0.241 \mathrm{~V}$ \\
\hline $2 \mathrm{H}^{+} / \mathrm{H}_{2}$ & (SHE) & $0.000 \mathrm{~V}$ \\
\hline $\mathrm{Ni}^{2+} / \mathrm{Ni}$ & & $-0.257 \mathrm{~V}$ \\
\hline $\mathrm{Co}^{2+} / \mathrm{Co}$ & & $-0.277 \mathrm{~V}$ \\
\hline $\mathrm{Fe}^{2+} / \mathrm{Fe}$ & & $-0.447 \mathrm{~V}$ \\
\hline $2 \mathrm{H}_{2} \mathrm{O} / \mathrm{H}_{2}+2 \mathrm{OH}^{-}$ & & $-0.828 \mathrm{~V}$ \\
\hline
\end{tabular}
standard hydrogen electrode (SHE), which is defined as $E_{00}\left(H / H^{+}\right)=0 \mathrm{~V}$.

Table 2.1: Standard potentials $E_{00}(r e d / o x)$ for $c=1 \mathrm{M}$ in solution [8, 25, 78]. Electrochemical potentials are often quoted versus the saturated calomel electrode (SCE) or the standard hydrogen electrode (SHE).

Thus, the calculated Nernst potential $E_{0}\left(C o / C^{2+}\right)$ for a $1 \mathrm{mM}$ cobalt(II) sulfate $\left(\mathrm{CoSO}_{4}\right)$ solution can be calculated to be

$$
\begin{aligned}
E_{0}\left(C o / \mathrm{Co}^{2+}\right) & =-0.277 \mathrm{~V}+0.0128 \mathrm{~V} \cdot \ln \left(10^{-3}\right) \\
& =-0.365 \mathrm{Vvs} \mathrm{SHE}
\end{aligned}
$$




\section{ELECTROCHEMISTRY}

Specific metal bulk deposition/dissolution onto the substrate can be accomplished by adjusting the potential of the working electrode (substrate) $E_{\mathrm{WE}}$ to values below/above the Nernst potential of the red/ox couple of interest $E_{0}\left(M / M^{z+}\right)$ :

$$
\begin{array}{ll}
\text { metal deposition : } & E_{\mathrm{WE}}<E_{0}\left(M / M^{z+}\right) \\
\text { metal dissolution : } & E_{\mathrm{WE}}>E_{0}\left(M / M^{z+}\right) .
\end{array}
$$

For some combinations of metal red/ox couple and metal substrate, an electrochemical deposition occurs on the substrate at a more positive potential with respect to the expected Nernst potential $E_{0}\left(M / M^{z+}\right)$ [32, 186]. Therefore, it is called underpotential deposition (UPD), since the cathodic potential $E_{\mathrm{WE}}$ is below $E_{0}\left(M / M^{z+}\right)$. However, when UPD occurs, only one monolayer, in special cases two monolayers [32], of the deposited metal is grown on the substrate, in contrast to the above mentioned metal bulk deposition which is also called overpotential deposition (OPD). After an UPD, further (bulk) deposition occurs at a more negative potential with respect to the expected Nernst potential $E_{0}\left(M / M^{z+}\right)$.

\subsubsection{The Solid/Liquid Interface}

At the interface between the metallic working electrode and the electrolyte a region is formed where electron and ion transfer take place [9, 78, 106]. This is illustrated in Figure 2.2, where the metallic working electrode at the solid/liquid interface is negatively charged (cathode), and thus a space charge region at the metal surface entails. Cations are adsorbed out of solution next to the metal surface compensating the charge at the metal surface. Thereby, a layer at the interface is formed by the metal space charge region on the one side and adsorbed ions on the other. By changing the metal potential to be positively charged with respect to the solution anions are adsorbed.

In a simplistic approach this interface between a metal bulk and an electrolyte bulk is comparable to a capacitor and was named double-layer by Helmholtz in 1853 [82, 83, 84]. Gouy and Chapman theoretically described the solid/liquid interface as a diffuse double-layer (DL) caused by thermal motion [37, 67, 68].

Both models were combined by Stern to the Gouy Chapman-Stern model in 1924 [182] and later modified by Grahame [70] and Bockris et al. [22]. Thus, the interface on the electrolyte side is divided into three regions which are illustrated in Figure 2.2 . The region ranging from the metal surface to the center of specifically adsorbed ions (here cations) is called the inner Helmholtz plane (I). Specifically adsorbed ions partially or totally lost their hydration shell and are in direct contact to the metal electrode. The outer Helmholtz plane (II) reaches to the center of still solvated ions, which are non-specifically adsorbed. While the inner Helmholtz plane (I) is a compact layer, the solvated cations of the outer Helmholtz (II) plane spread into the 


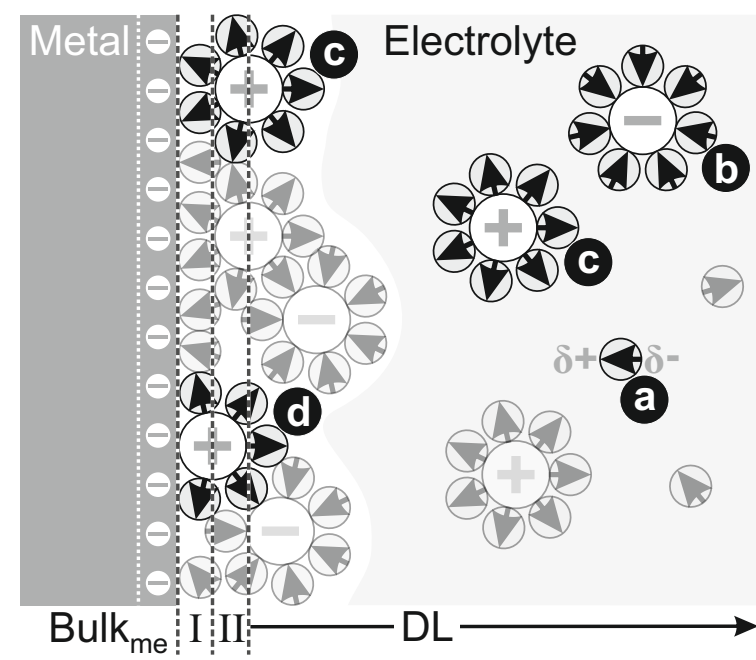

Figure 2.2: Solid/liquid interface model between a negatively polarized metal electrode and the electrolyte with (a) solvent molecules (water), where (b) anions and (c) cations are fully solvated [87]. The region ranging from the metal surface to the center of (d) specifically adsorbed cations is called the inner Helmholtz plane (I), whereas the outer Helmholtz plane (II) reaches to the center of (c) solvated cations. The diffuse layer (DL) is caused by thermal motion of the ions. $[9,78,106$.

electrolyte caused by thermal motion. Thus, next to the Helmholtz layer $(\mathrm{HL}=\mathrm{I}+\mathrm{II})$ a diffuse layer (DL) is formed extending into the electrolyte. Its thickness depends on the concentration of the ions in the electrolyte. To keep the extension of the diffuse layer low the electrolyte solution consists mainly of supporting electrolyte, which does not participate in any desired reactions, for example $\mathrm{CoSO}_{4}$ diluted in a supporting solution of $\mathrm{Na}_{2} \mathrm{SO}_{4}$.

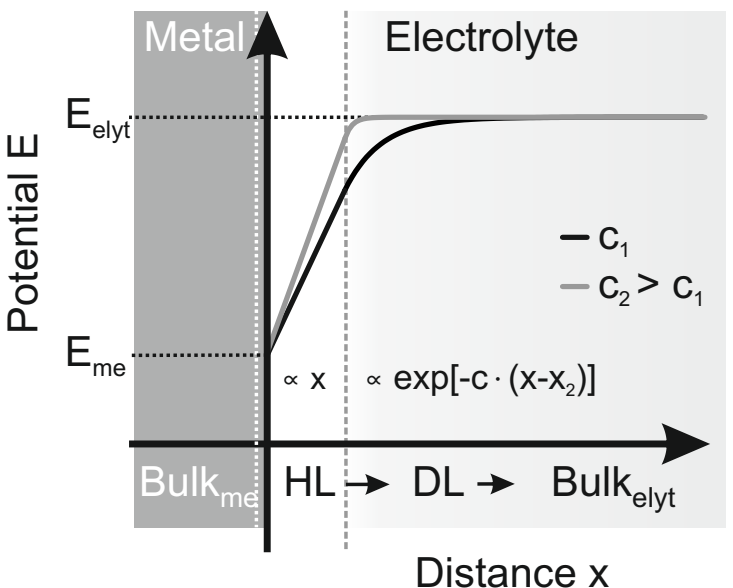

Figure 2.3: According to the Gouy Chapman Stern model a potential profile perpendicular to the electrode is divided into the compact Helmholtzlayer (HL) followed by a diffuse layer (DL). In the HL the potential increases linearly and converges exponentially in the DL to the potential value of the electrolyte depending on the electrolyte bulk concentration $c$.

The potential profile depending on the distance perpendicular to the metal surface is illustrated in Figure 2.3. The total capacitance of the double-layer is given in an equivalent electric circuit of two capacitors in series. According to the Gouy. Chapman-Stern model the profile from the potential of the metal bulk $\left(E_{\text {me }}\right)$ to the potential of the electrolyte bulk ( $E_{\text {elyt }}$, far away from the working electrode) is divided into two regions: the compact Helmholtz-layer (HL) followed by the diffuse layer (DL). In the Helmholtz-layer the potential increases linearly until the diffuse 


\section{ELECTROCHEMISTRY}

layer is reached, where an exponential convergence to the electrolyte potential value occurs. Since the exponential behavior depends on the concentration $c$ of the electrolyte bulk (concentration far away from the working electrode), the extension of the DL is lower for higher concentrations.

\subsection{Electrochemical Setup}

All components of the electrochemical cell are made of Duran glass or Teflon. Before and after use all components are stored in a 1:1-solution of hydrogen peroxide $\left(\mathrm{H}_{2} \mathrm{O}_{2}\right.$, $35 \%)$ and sulfuric acid $\left(\mathrm{H}_{2} \mathrm{SO}_{4}, 95-97 \%\right)$, known as Caro's acid. Thereby, organic contaminants, metallic oxides, and salts are removed from all components of the cell. The cleanliness of the cell is ensured through the use of triple distilled water for washing. All diluted solutions are prepared with triple distilled water and pure chemicals (see appendix A).

a
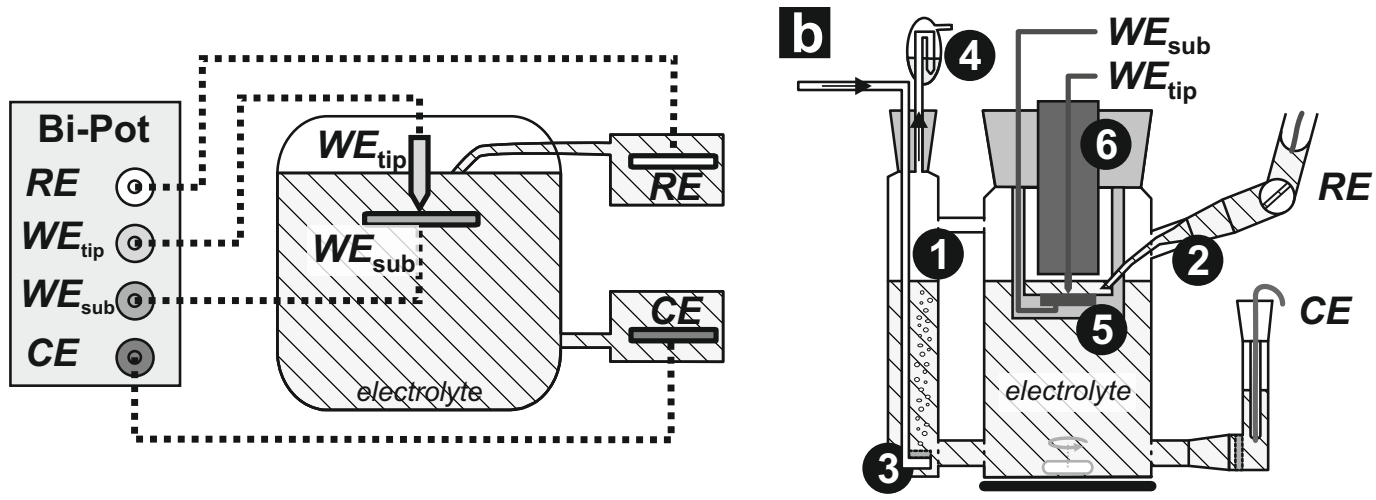

Figure 2.4: (a) Schematic of the electrochemical cell: Working electrodes are given by the STM tip $\left(W E_{\text {tip }}\right)$ and the investigated substrate $\left(W E_{\text {sub }}\right)$. The reference electrode $(R E)$ is embedded near both working electrodes using a Luggin capillary. Via the counter electrode $(C E)$ the current to adjust the potentials is given. A bipotentiostat (Bi-Pot) is used to control all potentials. (b) Section view of the electrochemical cell with a second deaeration column (1). Highlighted are the Luggin capillary (2), the porous frit (3), the airlock (4), the holder of the substrate (5), and the STM scanner unit (6). A magnetic stir bar is used to mix the electrolyte solution during deaeration.

The STM tip and the investigated substrate are positioned in the electrochemical cell and both act independently from each other as working electrodes, $W E_{\text {tip }}$ and $W E_{\text {sub }}$. Therefore, a setup of an electrochemical cell with four electrodes is required, as illustrated in Figure 2.4(a). The potentials of both working electrodes $W E_{\text {tip }}$ and $W E_{\text {sub }}$ are controlled independently by a bipotentiostat 11 [86]. Since the substrate

${ }^{1}$ BP-700 from EC-Tec 
is the main focus of the electrochemical investigation, it is commonly referred to as working electrode $W E$. A platinum wire acts as a counter electrode $(C E)$ to provide the required current. A saturated calomel electrode $(\mathrm{SCE})^{1}$ is used as a reference electrode $(R E)$ placed near the working electrodes using a Luggin capillary.

According to calibration measurements of this reference electrode, the potential of the used SCE appears to be $0.331 \mathrm{~V}$ vs SHE instead of $0.241 \mathrm{~V}$ vs SHE. Returning to the example for the Nernst potential of a $1 \mathrm{mM} \mathrm{CoSO}_{4}$ solution from Equation 2.5 the Nernst potential vs SCE in this case is

$$
\begin{aligned}
E_{0, \mathrm{SCE}}\left(C o / \mathrm{Co}^{2+}\right) & =E_{0, \mathrm{SHE}}\left(\mathrm{Co} / \mathrm{Co}^{2+}\right)-E_{00}(\text { calomel }) \\
& =-0.696 \mathrm{Vvs} \mathrm{SCE}
\end{aligned}
$$

Although a SCE is used in this electrochemical setup, all results are quoted with respect to SHE. Thereby, observations can be compared to values in literature such as standard potentials. Since $E_{00}($ calomel $)>E_{00}\left(H_{2} / 2 H^{+}\right)$all measured potentials vs SCE are shifted into the negative potential range compared to quotation vs SHE.

The electrochemical cell in use is airtight and contains approximately $450 \mathrm{ml}$ of the electrolyte solution. The oxygen is removed in-situ from the electrolyte and cell by deaeration with $5 \mathrm{~N}$ argon gas prior to each experiment. As illustrated in Figure 2.4(b) the argon gas passes through a porous frit (2), which acts as an inlet into the electrolyte, ascending through a second column of the cell (deaeration column). The displaced air/oxygen leaves the cell via an airlock (3). Using a magnetic stirrer the electrolyte solution in the cell is deaerated within $45 \mathrm{~min}$. The airtight seal ensures an electrochemical setup remaining deaerated during the following measurements, even after the magnetic stirrer is turned off [94].

\subsection{Characterization}

This home-built electrochemical setup [92, 94, 160] is developed further adding the deaeration column. Its performance is demonstrated in this section by cyclic voltammetry which is a powerful tool to investigate electrochemical reactions at the working electrode [9, 180], initially introduced showing measured UPD of $\mathrm{Cu}$ on $\mathrm{Au}(111)$ as an example. Resulting cyclic voltammograms (CVs) of $\mathrm{Au}(111)$ in a supporting electrolyte solution can be used to monitor quality and cleanliness of the electrochemical setup.

The $\mathrm{Au}(111)$ single crystal in use is always stored and cleaned in a bath of Caro's acid, to keep it free of contamination. Prior to each experiment a $\sqrt{3} \times 22$ reconstructed $\mathrm{Au}(111)$ surface is prepared by annealing the $\mathrm{Au}(111)$ single crystal in a hydrogen flame and subsequently cooling down in a nitrogen atmosphere [104, 105].

\footnotetext{
${ }^{1}$ The red/ox couple of a saturated calomel electrode is $\mathrm{Hg}_{2} \mathrm{Cl}_{2}+2 e^{-} \rightleftharpoons 2 \mathrm{Hg}+2 \mathrm{Cl}^{-}$and its standard potential $E_{0}$ (calomel) is $0.241 \mathrm{~V}$ vs SHE.
} 


\subsubsection{Cyclic Voltammetry}
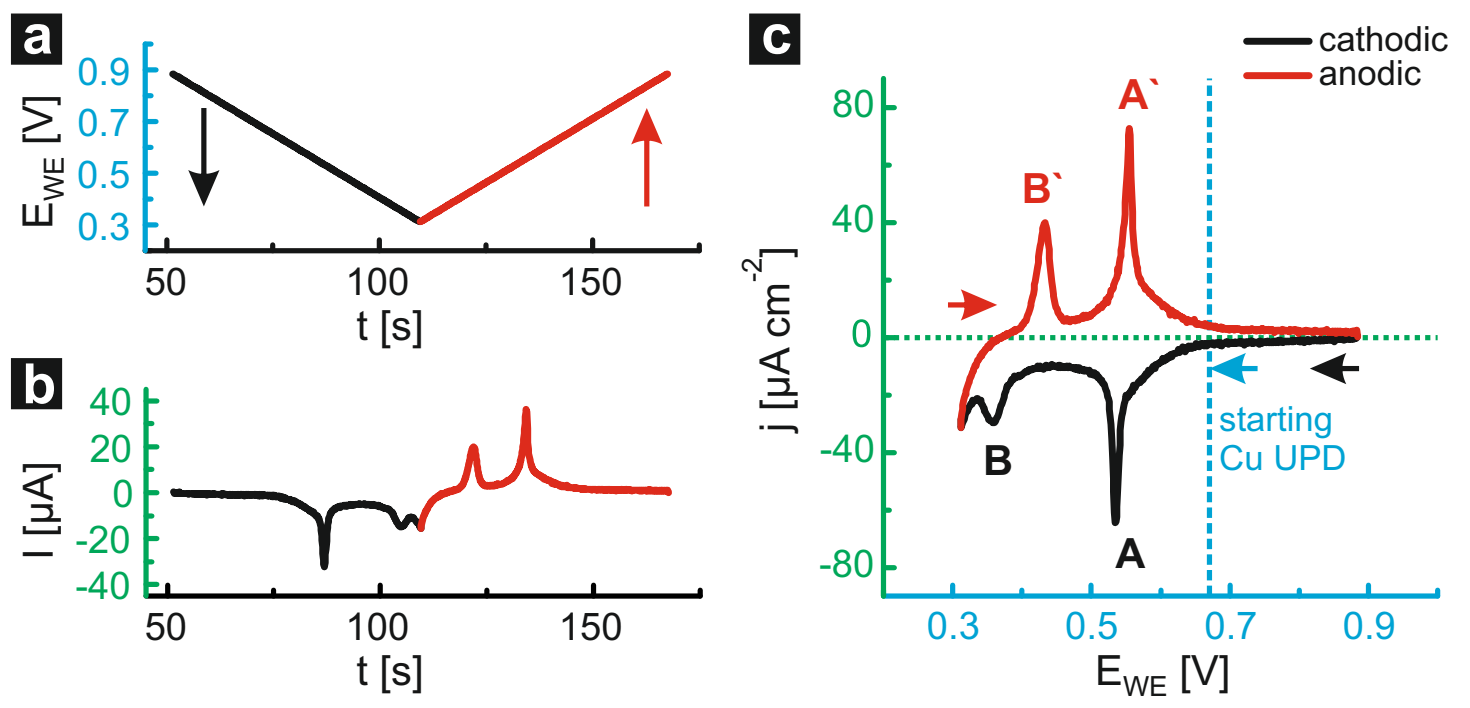

Figure 2.5: Example for cyclic voltammetry: UPD of $\mathrm{Cu}$ on $\mathrm{Au}(111)$ (a) Potential sweep of the working electrode $E_{\mathrm{WE}}$ with $\frac{d E_{\mathrm{WE}}}{d t}=10 \mathrm{mV} \mathrm{s}^{-1}$ in cathodic direction and reversed. (b) The corresponding current at the working electrode is measured in parallel and depends on electrochemical reactions. (c) Plotting the current density $j$ versus the potential $E_{\mathrm{WE}}$ results in the cyclic voltammogram (CV) of $\mathrm{Au}(111)$ in a solution of $1 \mathrm{mM} \mathrm{CuSO}_{4}$. The expected UPD of $\mathrm{Cu}$ on $\mathrm{Au}(111)$ starts at about $670 \mathrm{mV}$ observed in two steps by the deposition peaks $\mathrm{A}$ and $\mathrm{B}$. The corresponding dissolution is observable in the anodic current density around B' and A' for the anodic potential.

Cyclic voltammetry to observe a red/ox reaction is accomplished by sweeping the potential of the working electrode $E_{\mathrm{WE}}$ through more negative (cathodic) and subsequently more positive (anodic) regions with respect to the specific Nernst potential $E_{0}(\mathrm{red} / \mathrm{ox})$ at a fixed sweep rate $\frac{d E_{\mathrm{WE}}}{d t}$. Thereby, cathodic and anodic currents caused by all electrochemical reactions at the working electrode are measured. Plotting the current density $j=\frac{\text { current }}{\text { surface area }}$ versus the potential $E_{\mathrm{WE}}$ for both sweep direction results in the so called cyclic voltammogram (CV).

An example is given by a $\mathrm{CV}$ of $\mathrm{Cu}$ deposition/dissolution onto $\mathrm{Au}(111)$, plotted in Figure 2.5. The electrochemical behavior of the combination $\mathrm{Cu} / \mathrm{Au}(111)$ is well known [12, 74, 89, 119]. Since $\mathrm{Cu} / \mathrm{Au}(111)$ shows UPD, $\mathrm{Cu}$ deposition on $\mathrm{Au}(111)$ starts at a more positive potential of the $\mathrm{Au}(111)$ working electrode $E_{\mathrm{WE}}$ than expected by the calculated Nernst potential $E_{0}\left(C u / C u^{2+}\right)=253 \mathrm{mV}$ vs SHE of a $1 \mathrm{mM}$ copper sulfate $\left(\mathrm{CuSO}_{4}\right)$ solution. The supporting electrolyte solution is composed of $50 \mathrm{mM}$ sodium sulfate $\left(\mathrm{Na}_{2} \mathrm{SO}_{4}\right)$ and $0.1 \mathrm{mM}$ sulfuric acid $\left(\mathrm{H}_{2} \mathrm{SO}_{4}\right)$. 
The potential sweep rate $\frac{d E_{\mathrm{WE}}}{d t}$ is set to $10 \mathrm{mV} \mathrm{s}^{-1}$, as plotted versus time in Figure 2.5(a). For one CV a circle sweep in downward and upward direction is required to investigate $\mathrm{Cu}$ deposition and subsequent $\mathrm{Cu}$ dissolution. Thereby, the corresponding cathodic/anodic current at the $\mathrm{Au}(111)$ working electrode caused by $\mathrm{Cu}$ deposition/dissolution is measured, as shown in Figure 2.5(b). The resulting CV, which is shown in Figure 2.5(c), indicates two cathodic peaks A and B in the downwards sweep (decreasing potential) and their related anodic peaks A' and B' in the upwards sweep (increasing potential).

The observed behavior can be attributed to the UPD of $\mathrm{Cu}$ on $\mathrm{Au}(111)$, where an initial monolayer of $\mathrm{Cu}$ is already deposited before the bulk deposition of $\mathrm{Cu}$ on $\mathrm{Au}(111)$ occurs. The observed $\mathrm{Cu}$ UPD starts roughly at $670 \mathrm{mV}$, which results in an increasing cathodic current density. The $\mathrm{Cu}$ deposition takes place in two steps around $E_{\mathrm{WE}}=534 \mathrm{mV}(\mathrm{A})$ and around $E_{\mathrm{WE}}=360 \mathrm{mV}(\mathrm{B})$, displayed by the peaks $\mathrm{A}$ and $\mathrm{B}$ of the cathodic current density. At $E_{\mathrm{WE}} \approx 330 \mathrm{mV}$ the cathodic current density decreases again, since the initial $\mathrm{Cu}$ monolayer is completed before the $\mathrm{Cu}$ OPD starts.

At around $205 \mathrm{mV}(\mathrm{A})$ the deposited $\mathrm{Cu}$ is arranged on $\mathrm{Au}(111)$ in a $(\sqrt{3} \times$ $\sqrt{3}) R 30^{\circ}$ structured layer which is completed with decreasing potential, thus the cathodic current density decreases again and the cathodic peak A is observed. Since a phase transition of the deposited $\mathrm{Cu}$ on the $\mathrm{Au}(111)$ surface from a $(\sqrt{3} \times \sqrt{3}) R 30^{\circ}$ structure to a closer packed $(1 \times 1)$ structure occurs around $360 \mathrm{mV}$, additionally deposited $\mathrm{Cu}$ leads to an increasing current density until the $(1 \times 1)$ structure is completely formed. Both corresponding $\mathrm{Cu}$ dissolution peaks are found in the anodic current density around $E_{\mathrm{WE}}=433 \mathrm{mV}$ (B') and $E_{\mathrm{WE}}=555 \mathrm{mV}$ (A'), where the phase transformation is measured accordingly.

Therefore, using a CV to investigate a metal red/ox reaction on a working electrode, an increasing cathodic current density represents the metal reduction (deposition). Subsequently, the related increasing anodic current density represents the metal dissolution.

The observed deposition/dissolution peaks for a $\mathrm{Cu}$ monolayer on $\mathrm{Au}(111)$ in the range of $30 \mu \mathrm{A} \mathrm{cm}^{-2}$ to $80 \mu \mathrm{A} \mathrm{cm}^{-2}$ can easily be investigated by cyclic voltammetry. This accentuates the level of sensitivity of this electrochemical method and its advantage for further electrochemical investigations.

\subsubsection{Deaeration of the Electrolyte}

In all aqueous solutions the potential range is limited by the hydrogen evolution at negative potentials and by oxygen evolution at positive potentials [108, 143] which are observed by cathodic/anodic increases of the current density at these potentials. This behavior is unavoidable and occurs in all aqueous solutions.

Additionally, oxygen is dissolved in all aqueous solutions [101] [110], and is thus 


\section{ELECTROCHEMISTRY}
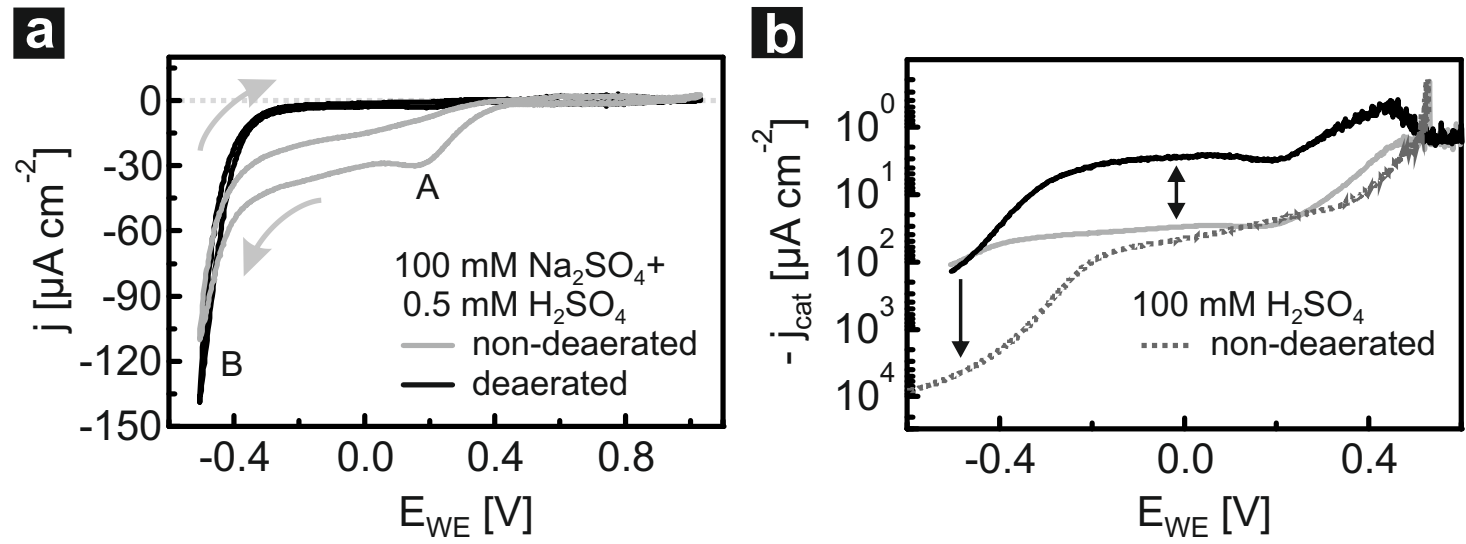

Figure 2.6: $\mathrm{CVs}$ of $\mathrm{Au}(111), \frac{d E_{\mathrm{WE}}}{d t}=10 \mathrm{mV} \mathrm{s}^{-1}$ : (a) Deaeration of the supporting electrolyte is demonstrated by comparing CVs of $\mathrm{Au}(111)$ in $0.1 \mathrm{M} \mathrm{Na}_{2} \mathrm{SO}_{4}$ with $0.5 \mathrm{mM}$ $\mathrm{H}_{2} \mathrm{SO}_{4}$ before and after deaeration. The $\mathrm{CV}$ in a deaerated electrolyte (black) shows only an increase of the cathodic current density with decreasing potential, caused by hydrogen evolution (B). Compared to this, a CV in a non-deaerated electrolyte (gray) shows, that dissolved oxygen causes higher current densities (A) in the potential range below $500 \mathrm{mV}$. (b) Using a logarithmic scale the difference between a deaerated and a non-deaerated electrolyte becomes obvious. A non-deaerated supporting electrolyte of $0.1 \mathrm{M} \mathrm{H}_{2} \mathrm{SO}_{4}$ (dashed) instead of $\mathrm{Na}_{2} \mathrm{SO}_{4}$ (solid) causes a much stronger hydrogen evolution.

also observed in CVs of a non-deaerated aqueous electrolyte. An example of such an aqueous solution with dissolved oxygen is shown in Figure 2.6(a) by the CV of $\mathrm{Au}(111)$ (gray line). There, the reduction of $\mathrm{O}_{2}$ to $\mathrm{H}_{2} \mathrm{O}_{2}$ and $\mathrm{H}_{2} \mathrm{O}$ causes an increase of the cathodic current density (around mark A) in the negative potential range starting from $500 \mathrm{mV}$ [8]. However, since the deposition of ferromagnetic metals such as $\mathrm{Co}, \mathrm{Fe}$, and $\mathrm{Ni}$ takes place in this potential range (see Table 2.1) any parasitic and undesired reactions have to be avoided. Investigations on such electrochemically deposited metals can be accomplished only after a deaeration of the aqueous solution.

Deaeration is accomplished in-situ with $5 \mathrm{~N}$ argon gas for $45 \mathrm{~min}$ prior to all measurements [208] using the magnetic stirrer. This treatment causes a decrease of oxygen within and above the electrolyte. Thereby, related undesired reactions decrease, which is made obvious in Figure 2.6(a) by the CV of $\mathrm{Au}(111)$ subsequent to the deaeration (black line). Displaying the current density on a logarithmic scale such as in Figure 2.6(b), is commonly known as a Tafel plot and facilitates the observation of small and large currents alike. This presentation highlights the difference between a deaerated and a non-deaerated electrolyte.

Special attention is given to the hydrogen evolution. Two CVs of $\mathrm{Au}(111)$ in a 
supporting solution of $0.1 \mathrm{M} \mathrm{Na}_{2} \mathrm{SO}_{4}$ with $0.5 \mathrm{mM} \mathrm{H}_{2} \mathrm{SO}_{4}$ (solid line) or $0.1 \mathrm{M} \mathrm{H}_{2} \mathrm{SO}_{4}$ (dashed line) are shown in Figure 2.6(b). Caused by the higher concentration of $\mathrm{H}^{+}$ cations in solution, a much stronger hydrogen evolution occurs at the working electrode. Nevertheless, this behavior can be strongly reduced by using $0.1 \mathrm{M} \mathrm{Na}_{2} \mathrm{SO}_{4}$ as supporting electrolyte.

\subsubsection{Cleanliness of the Electrochemical Environment}

The cleanliness of the electrochemical environment can be demonstrated simply by a CV of a freshly prepared $\mathrm{Au}(111)$ surface in a supporting electrolyte of $0.1 \mathrm{M}$ $\mathrm{Na}_{2} \mathrm{SO}_{4}$ and $0.5 \mathrm{mM} \mathrm{H}_{2} \mathrm{SO}_{4}$, as plotted in Figure 2.7.

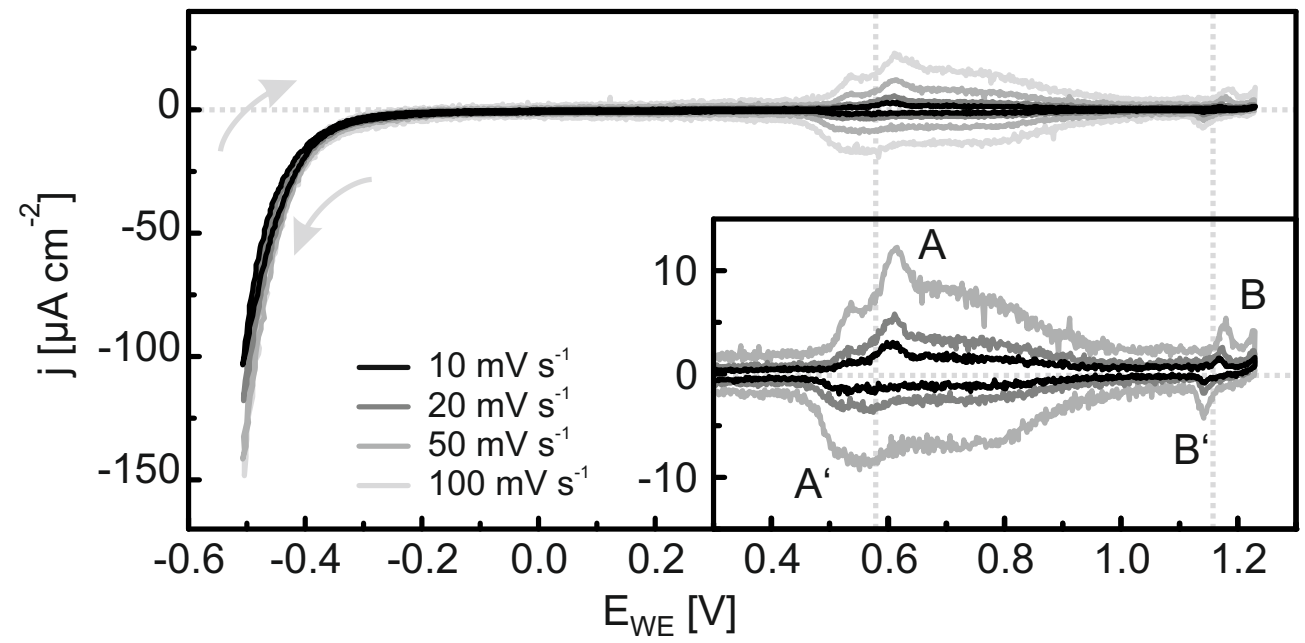

Figure 2.7: Cleanliness of the electrochemical cell is demonstrated by CVs of $\mathrm{Au}(111)$ in a deaerated electrolyte of $0.1 \mathrm{M} \mathrm{Na}_{2} \mathrm{SO}_{4}$ and $0.5 \mathrm{mM} \mathrm{H}_{2} \mathrm{SO}_{4}$ for various sweep rates $\frac{d E_{\mathrm{WE}}}{d t}$. In the higher potential range only the expected adsorption $(\mathrm{A}) / \operatorname{desorption}\left(\mathrm{A}^{\prime}\right)$ of $\mathrm{SO}_{4}^{2-}$ anions on the $\mathrm{Au}(111)$ surface is observed. A phase transition of the adsorbed anions from a disordered and mobile structure to an ordered adlayer causes peak B to arise [43, 151, 104, 117, 120]. Inevitably, hydrogen evolution arises at low potentials, reflected by a steep increase of the cathodic current density.

A contamination of the electrochemical environment can be observed by this highly sensitive measurement. Therefore, the CV is measured between $-850 \mathrm{mV}$ and $900 \mathrm{mV}$ to investigate the full potential range in an aqueous electrolyte. Several CVs are recorded for various sweep rates $\left(10 \mathrm{mV} \mathrm{s}^{-1}, 20 \mathrm{mV} \mathrm{s}^{-1}, 50 \mathrm{mV} \mathrm{s}^{-1}\right.$ and $100 \mathrm{mV} \mathrm{s}^{-1}$ ) to observe all kinds of electrochemical reactions, even if only small amounts of contamination are involved.

By the CVs two peaks ( $\mathrm{A}$ and $\mathrm{B}$ ) are observed in the positive potential range of the anodic current densities. These correspond to a $\mathrm{SO}_{4}^{2-}$ anion induced lifting 


\section{ELECTROCHEMISTRY}

of the $\sqrt{3} \times 22$ surface reconstruction of the $\mathrm{Au}(111)$ single crystal to a $1 \times 1$ closepacked $\mathrm{Au}(111)$ surface (peak A) [43, 51, 104, 117, 120. Peak B represented the transformation from a disordered sulfate adlayer to an ordered $\sqrt{3} \times \sqrt{7}$ structure on the $1 \times 1 \mathrm{Au}(111)$ surface. The lifting of the sulfate adlayer and rearrangement of the $\sqrt{3} \times 22$ reconstructed $\mathrm{Au}(111)$ surface are observed at the cathodic peaks B' and A'.

Figure 2.8: A logarithmic presentation of the CVs, similar to the one shown in Figure 2.7. Small increases/decreases can be noticed in the cathodic current density $j_{\text {cat }}$. The desorption of the $\mathrm{SO}_{4}^{2-}$ anions and the rearrangement of the $\sqrt{3} \times 22 \mathrm{Au}(111)$ reconstruction is observed by peak B' and A', respectively. Hydrogen evolution can be linearly fitted (dashed line). Even using this kind of presentation no appreciable contamination is observed.

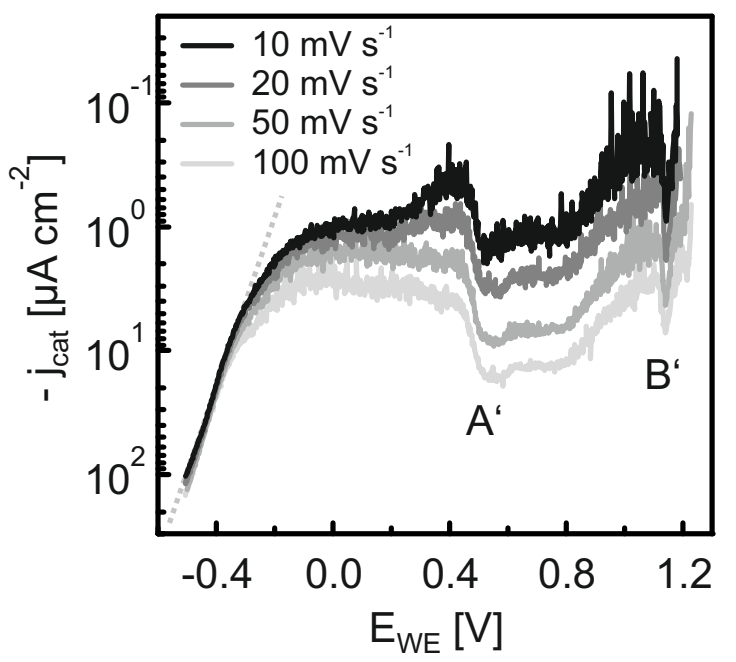

In order to better observe smaller values of the current density a Tafel plot is used for the CVs of $\mathrm{Au}(111)$ for various sweep rates, as shown in Figure 2.8. Besides the desorption of the $\mathrm{SO}_{4}^{2-}$ anions and the rearrangement of the $\sqrt{3} \times 22 \mathrm{Au}(111)$ the increase caused by hydrogen evolution is observed and can be linearly fitted. For increasing sweep rates $\frac{d E_{\mathrm{WE}}}{d t}$ a higher cathodic current density arises since the charge passed must remain constant [210].

No additional increase of the current density is observed due to the absence of noteworthy impurities. Thus, it is clearly shown that no appreciable concentrations of contaminants are found within the electrochemical environment.

\subsection{Deposition of Co on $\mathrm{Au}(111)$}

The electrochemical Co deposition on an $\mathrm{Au}(111)$ single crystal is discussed in the following section. Only OPD is expected for Co deposition on $\mathrm{Au}(111)$ in a supporting electrolyte of sodium sulfate $\left(\mathrm{Na}_{2} \mathrm{SO}_{4}\right)$ and sulfuric acid $\left(\mathrm{H}_{2} \mathrm{SO}_{4}\right)$, as opposed to $\mathrm{Cu}$ deposition on $\mathrm{Au}(111)$. Furthermore, hydrogen evolution takes place at the working electrode caused by the low Nernst potential of $E_{0}\left(\mathrm{Co}_{\mathrm{CO}} \mathrm{Co}^{2+}\right)=-365 \mathrm{mV}$ for a $1 \mathrm{mM}$ cobalt sulfate $\left(\mathrm{CoSO}_{4}\right)$ solution. In order to prevent this competing reaction, hydrogen evolution is minimized by use of $0.1 \mathrm{M}$ sodium sulfate $\left(\mathrm{Na}_{2} \mathrm{SO}_{4}\right)$ 
as supporting electrolyte solution instead of $0.1 \mathrm{M}$ sulfuric acid $\left(\mathrm{H}_{2} \mathrm{SO}_{4}\right)$. All CVs shown in the previous Sections 2.3 .2 and 2.3 .3 were realized already for such an aqueous solution.

In Pourbaix diagrams equilibrium potentials of various oxidation states of an element are plotted versus $\mathrm{pH}$-value. Thereby, it is shown for the $\mathrm{Co} / \mathrm{H}_{2} \mathrm{O}$ system, that red/ox reactions of Co compounds are influenced by the $\mathrm{pH}$-value of the aqueous solution [59, 122, 147].

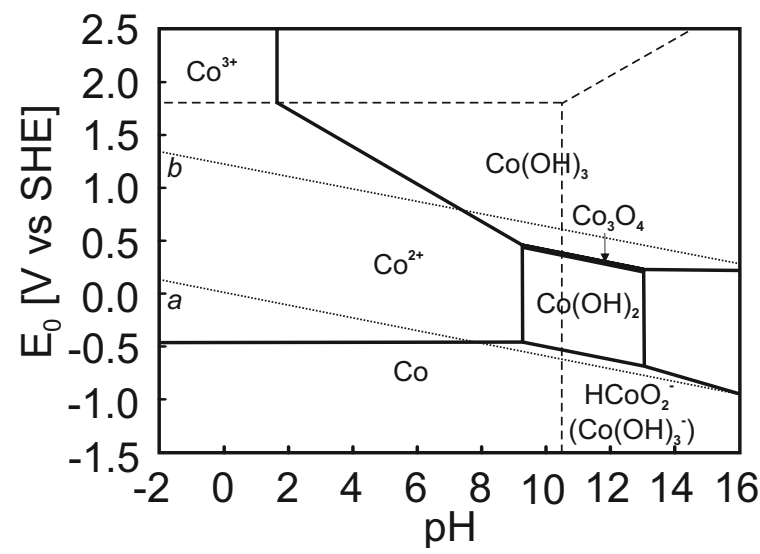

Figure 2.9: Pourbaix diagram for the $\mathrm{Co} / \mathrm{H}_{2} \mathrm{O}$ system where the Nernst potentials for stable phases are plotted vs $\mathrm{pH}$-value. The dashed lines $a$ and $b$ show the stability limits of water. In the alkaline range the formation of $\mathrm{Co}$ hydroxide complexes occurs.

From Garcia et al. [59] with modifications.

Thus, before the deposition of $\mathrm{Co}$ on $\mathrm{Au}(111)$ is discussed in detail, in the following some electrochemical investigations are presented regarding the concentration of $\mathrm{H}^{+}$cations at the working electrode, and thus, the local $\mathrm{pH}$-value.

\subsubsection{Shift of $\mathrm{pH}$-value}

In general the mass transport of reactants in an electrolyte solution, for example $\mathrm{H}^{+}$or $\mathrm{Co}^{2+}$ cations, is driven by diffusion (movement against the concentration gradient), migration (movement of charged ions in an electric field), and convection (movement caused by density gradients or stirring). The flux of a reactant in one dimension is expressed by the Nernst-Planck equation [210]:

$$
J(x)=-D \frac{\delta C(x)}{\delta x}-\frac{z F}{R T} D C \frac{\delta \phi(x)}{\delta x}+C v(x)
$$

$z$ : charge

$D$ : diffusion coefficient of the reactant in solution

$C^{*}$ : concentration of the reactant in solution

$v(x)$ : movement of a volume element in solution

$\frac{\delta C(x)}{\delta x}$ : concentration gradient

$\frac{\delta \phi(x)}{\delta x}$ : potential gradient 


\section{ELECTROCHEMISTRY}

For a diffusion controlled movement the relation between the current density $j$ and the concentration of the reactant in solution $C^{*}$ is given by the Cottrell equation [210]

$$
j(t)=\frac{n F C^{*} \sqrt{D}}{\sqrt{\pi}} \cdot \frac{1}{\sqrt{t}}=a \cdot \frac{1}{\sqrt{t}} .
$$

By plotting the transient density current versus the variable $\frac{1}{\sqrt{t}}$ the current density can be fitted linearly, where the slope $a$ of this linear fit is equal to the factor $\frac{n F C^{*} \sqrt{D}}{\sqrt{\pi}}$. Therefore, the diffusion coefficient $\mathrm{D}$ of the reactant is

$$
D=\frac{a^{2} \pi}{\left(n F C^{*}\right)^{2}} .
$$

The concentration $C^{*}$ of the reactant in solution has to be known precisely in order to specify the diffusion coefficient D.

Diffusion of $\mathrm{H}^{+}$cations at the $\mathrm{Au}(111)$ working electrode $W E$ is experimentally demonstrated by the observed transient of the current density for various electrolyte solutions, as shown in Figure 2.10. Thereby, at the working electrode a potential sweep to $E_{\mathrm{WE}}=-480 \mathrm{mV}$ is adjusted, below the Nernst potential of $\mathrm{Co} / \mathrm{Co}^{2+}$, as shown in Figure 2.10(a). The corresponding current density is plotted in Figure 2.10(b). During these electrochemical measurements the electrolyte solution is not stirred, since the diffusion coefficient $D$ is being investigated. Additionally, this kind of potential sweep is similar to later performed sample preparation during STM investigations when stirring of the electrolyte is not possible as well.

Experiments are performed with various electrolyte solutions I, II, and III:

solution I: $\quad 0.1 \mathrm{M} \mathrm{Na}_{2} \mathrm{SO}_{4}$

solution II: $\quad 0.1 \mathrm{M} \mathrm{Na}_{2} \mathrm{SO}_{4}+0.5 \mathrm{mM} \mathrm{H}_{2} \mathrm{SO}_{4}$

solution III: $0.1 \mathrm{M} \mathrm{Na}_{2} \mathrm{SO}_{4}+0.5 \mathrm{mM} \mathrm{H}_{2} \mathrm{SO}_{4}+1 \mathrm{mM} \mathrm{CoSO}_{4}$

Solution I contains $0.1 \mathrm{M} \mathrm{Na}_{2} \mathrm{SO}_{4}$ as supporting electrolyte, whereas in solution II $0.5 \mathrm{mM} \mathrm{H}_{2} \mathrm{SO}_{4}$ is added, leading to $p H \approx 3$. Both solutions (I and II) show a decreasing current density over time after reaching the final potential, marked by the gray background. But the higher $\mathrm{H}^{+}$cation concentration in solution II causes a stronger hydrogen evolution and accordingly a larger current density throughout the entire measurement.

In addition, a third solution (III) is investigated containing $0.1 \mathrm{M} \mathrm{Na}_{2} \mathrm{SO}_{4}$, $0.5 \mathrm{mM} \mathrm{H}_{2} \mathrm{SO}_{4}$ and $1 \mathrm{mM} \mathrm{CoSO}_{4}$. Initially, almost the same hydrogen evolution is measured in solutions II and III demonstrated by the transient of the current 

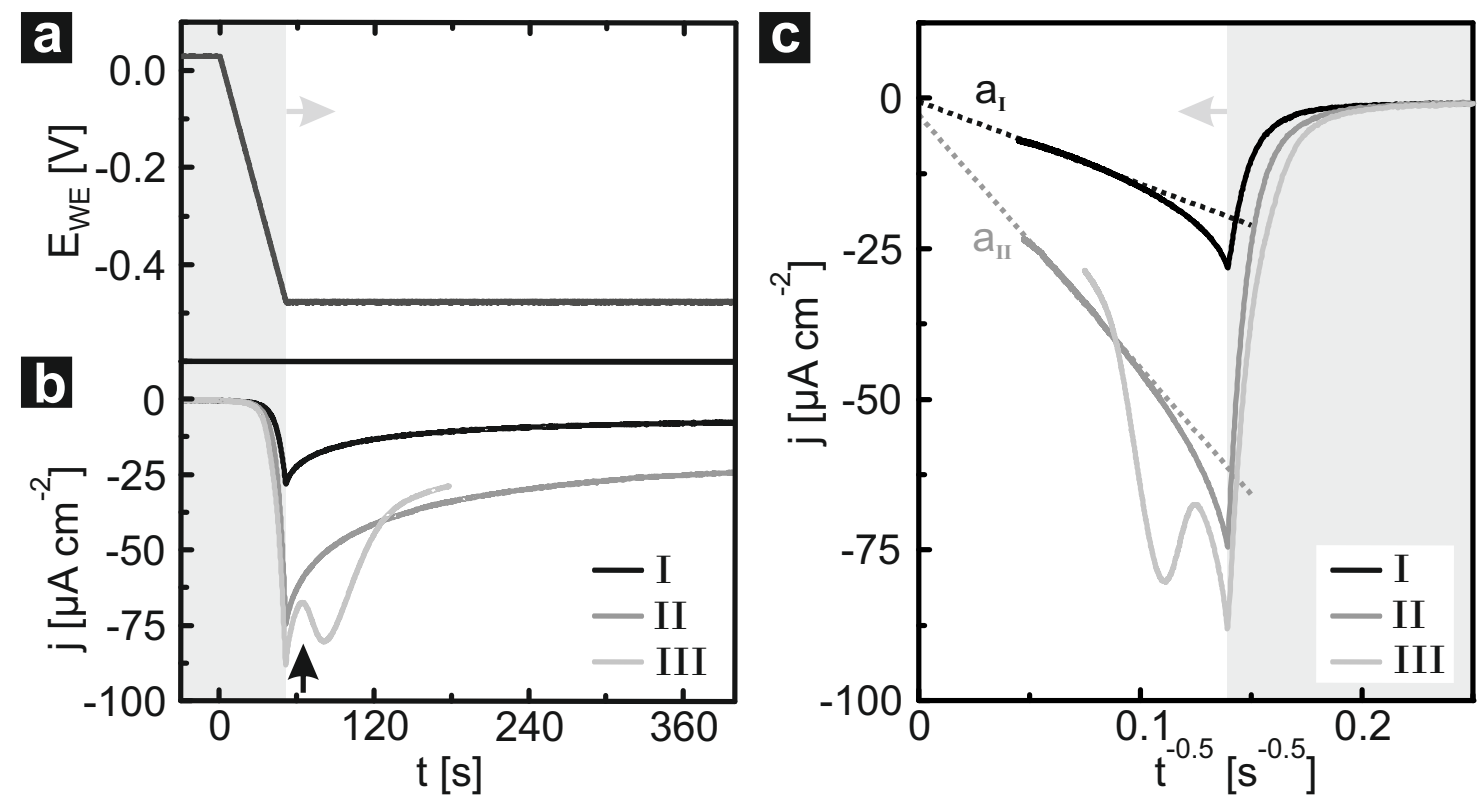

Figure 2.10: Investigation of the diffusion coefficient $D_{H^{+}}$(a) Potential sweep with $\frac{d E_{\mathrm{WE}}}{d t}=10 \mathrm{mV} \mathrm{s}^{-1}$ towards $E_{\mathrm{WE}}=-480 \mathrm{mV}$. Such a potential sweeps is similar to later performed Co deposition during STM measurements. (b) The corresponding current density $j(t)$ is measured for various solutions $\mathbf{I}: \mathrm{Na}_{2} \mathrm{SO}_{4}$ (supporting electrolyte), II: $\mathrm{I}+\mathrm{H}_{2} \mathrm{SO}_{4}$ (supporting electrolyte with $p H \approx 3$ ), III: $\mathrm{II}+\mathrm{CoSO}_{4}\left(\right.$ containing $\mathrm{Co}^{2+}$ ). Decreasing current densities indicate a decreasing hydrogen evolution for solutions $\mathbf{I}$ and II. A higher hydrogen evolution is measured in solution II due to $p H \approx 3$. The competing hydrogen evolution during Co deposition is observed in solution III by the delayed peak (black arrow). (c) The slope $a_{x}$ of a linear fit can be used to calculate the diffusion coefficient $D_{H^{+}}$for solution $x=\mathbf{I}, \mathbf{I I}$.

densities. For solution III, the Co deposition is presented by the cathodic peak of the current density. As a guide to the eye, the onset of the Co deposition is marked by the black arrow in Figure 2.10(b). It takes some seconds until the Co deposition starts, since the hydrogen reduction acts as a competing reaction at the working electrode and therefore hinders the Co deposition [141]. Therefore, the Co deposition is delayed. Since Co acts as chemical catalyst for hydrogen evolution [114, 141], the cathodic current density measured for solution III is not just attributed to the Co deposition, but also to a stronger hydrogen evolution. The further decrease in current density reveals a depletion at the working electrode of both $\mathrm{Co}^{2+}$ and $\mathrm{H}^{+}$ cations caused by their low concentration. The crossing between the current densities of solution II and III seems to result from the catalyzed hydrogen evolution. Thereby, a depletion of $\mathrm{H}^{+}$cations arises faster. 


\section{ELECTROCHEMISTRY}

The transient of each current density $j\left(\frac{1}{\sqrt{t}}\right)$ is plotted in Figure 2.10 (c). The linear behavior at higher time-values demonstrates the diffusion controlled response of the current density, as shown in Equation 2.8. Thereby, the diffusion coefficient $D_{H^{+}}$in solution I can be estimated to be $7.5 \cdot 10^{-5} \mathrm{~cm}^{2} \mathrm{~s}^{-1}$. This observed value is comparable with values in literature [116, 210].

Since the mass transport of the $\mathrm{H}^{+}$cations is diffusion controlled, a depletion of them at the $\mathrm{Au}(111)$ working electrode is observed, as reflected in the decreasing hydrogen evolution over time (Figure 2.10 b). The $\mathrm{H}^{+}$cations depletion and thus an increasing $\mathrm{pH}$-value is especially important for the desired deposition of Co on $\mathrm{Au}(111)$. A concentration profile of the $\mathrm{H}^{+}$cations in solution with increasing distance $x$ from the planar $\mathrm{Au}(111)$ working electrode $W E$ and depending on the time is given by

$$
C_{H^{+}}(x, t)=C_{H^{+}}^{*}\left[1-\operatorname{erfc}\left(\frac{x}{2 \sqrt{D t}}\right)\right],
$$

[210]. Some calculated concentration profiles $C_{H^{+}}(x, t)$ normalized to the adjusted concentration $C_{H^{+}}^{*}$ are plotted in Figure 2.11(a) for various time values by use of the estimated diffusion coefficient $D_{H^{+}}=7.5 \cdot 10^{-5} \mathrm{~cm}^{2} \mathrm{~s}^{-1}$. The concentration profiles

a

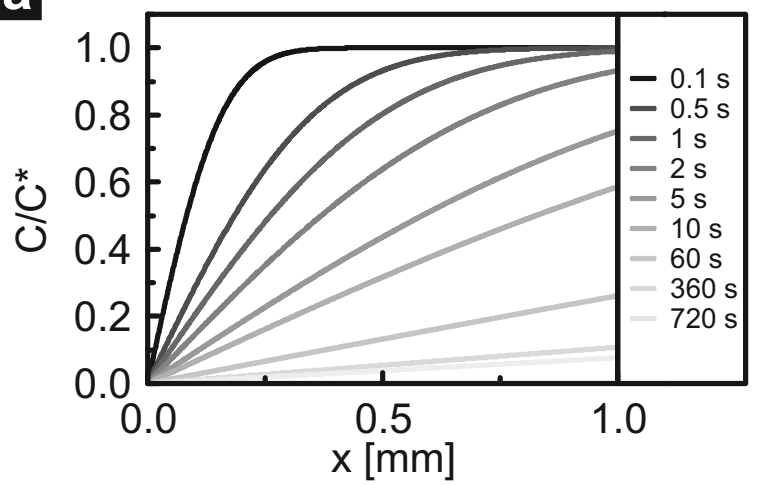

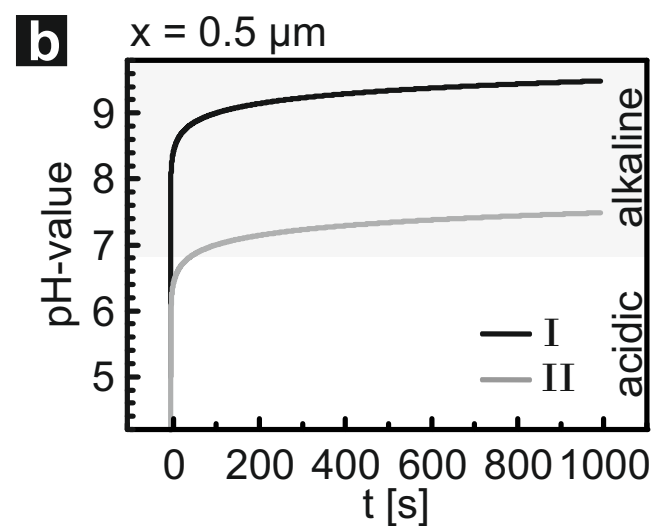

Figure 2.11: (a) Normalized concentration profiles $\frac{C(x, t)}{C^{*}}=\left[1-\operatorname{erfc}\left(\frac{x}{2 \sqrt{D t}}\right)\right]$ are calculated for various time values $t$, using $D_{H^{+}}=7.5 \cdot 10^{-5} \mathrm{~cm}^{2} \mathrm{~s}^{-1}$, see Equation 2.9 . (b) Calculated $\mathrm{pH}$-value depending on time $-\log \left(C_{H^{+}}(t, x=0.5 \mu \mathrm{m})\right)$ using $D_{H^{+}}=7.5 \cdot 10^{-5} \mathrm{~cm}^{2} \mathrm{~s}^{-1}$. Over time, in solution $\mathbf{I}\left(C_{H^{+}}^{*} \approx 0.01 \mathrm{mM}\right)$ the $\mathrm{pH}-$ value increases from the acidic range to the alkaline range until the diffusion of $\mathrm{H}^{+}$ cations from the solution is in balance. In solution $\mathbf{I I} C_{H^{+}}^{*}$ is supposed to be $1 \mathrm{mM}$, and thus the $\mathrm{pH}$-value is significantly shifted towards the acidic range.

change depending on the distance $x$ perpendicular to the working electrode until the bulk concentration of the solution is reached $\left(C / C^{*}=1\right)$. Regarding the changing profiles of various time values, it is shown that this balance is reached 
at larger distances for larger time values. In addition, calculations of the $\mathrm{pH}$-value depending on time $-\log \left(C_{H^{+}}(t, x)\right)$ are plotted in Figure 2.11(b) for solution I with $C_{H^{+}}^{*}=0.01 \mathrm{mM}$ and solution II with $C_{H^{+}}^{*}=1 \mathrm{mM}$. Here, the distance $x$ is set to $0.5 \mathrm{\mu m}$ perpendicular to the working electrode. Thereby, near to the working electrode in solution $\mathbf{I}$ a shift of the $\mathrm{pH}$-value from the acidic range starting from $\mathrm{pH}=5$ to the alkaline range $\mathrm{pH}=9.5$ is illustrated. Adding $\mathrm{H}_{2} \mathrm{SO}_{4}$ to the supporting electrolyte significantly reduces the $\mathrm{pH}$-value of the final state, as demonstrated by solution II.

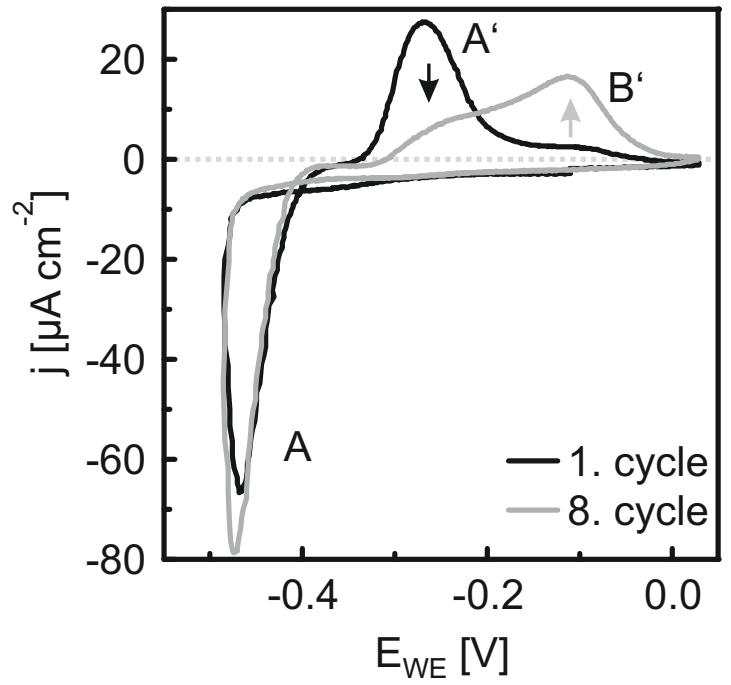

Figure 2.12: $\mathrm{CVs}$ of $\mathrm{Au}(111)$ in $40 \mathrm{mM}$ $\mathrm{Na}_{2} \mathrm{SO}_{4}$ and $1 \mathrm{mM} \mathrm{CoSO}_{4}$, but without $\mathrm{H}_{2} \mathrm{SO}_{4}$ in solution. Changes between the first $\mathrm{CV}$ and eighth $\mathrm{CV}$ are observed due to the shifting $\mathrm{pH}$-value from acidic to alkaline electrolyte at $W E$. Initially, Co dissolution takes place at peak A' around $-600 \mathrm{mV}$. Over time peak A' decreases and peak B' increases, which is attributed to the development of cobalt hydroxides 109, 145.

Since all red/ox reactions at a working electrode depend on the actual $\mathrm{pH}$-value [8, 59], the observed $\mathrm{CV}$ of $\mathrm{Au}(111)$ in an electrolyte composed of $\mathrm{Na}_{2} \mathrm{SO}_{4}$ and $\mathrm{CoSO}_{4}$, without adding $\mathrm{H}_{2} \mathrm{SO}_{4}$, is changed over time due to the increasing $\mathrm{pH}$ value [72, 109, 122, 125, 145]. This is demonstrated in Figure 2.12 by the fist cycle of the CV and the eighth. In an ideal system one expects all CVs to be equal, if the electrochemical conditions are stable at the working electrode. As demonstrated above, the environment at the working electrode becomes increasingly alkaline due to the depletion of $\mathrm{H}^{+}$cations near the $\mathrm{Au}(111)$ working electrode. Co deposition is sensitive to the actual $\mathrm{pH}$-value at the working electrode, and thus at $\mathrm{pH}$-values above 9, cobalt hydroxides such as $\mathrm{Co}(\mathrm{OH})_{2}$ develop and coexist [145]. The formation of these Co complexes can be seen as a green precipitation above the $\mathrm{Au}(111)$ working electrode.

Consequently, for Co deposition on $\mathrm{Au}(111)$ two opposite aspects have to be taken into account. First of all, the competing hydrogen evolution during Co deposition should be reduced to avoid a delay of the Co deposition. This is accomplished by use of sodium sulfate $\left(\mathrm{Na}_{2} \mathrm{SO}_{4}\right)$ as supporting electrolyte instead of sulfuric acid $\left(\mathrm{H}_{2} \mathrm{SO}_{4}\right)$. However, it is necessary to add a small amount of $\mathrm{H}_{2} \mathrm{SO}_{4}$ to the electrolyte 


\section{ELECTROCHEMISTRY}

solution. Thereby, the $\mathrm{pH}$-value at $W E$ is kept near the acidic range during and after Co deposition. Otherwise, stable complex formations of Co hydroxides occur at the working electrode and Co deposition/dissolution is not possible any more. Thus, the electrolyte solution has to contain $\mathrm{H}_{2} \mathrm{SO}_{4}$ to adjust the pH-value to 3 in order to investigate metallic $\mathrm{Co}$ on $\mathrm{Au}(111)$ by STM and later by sp-STM at the solid/liquid interface.

\subsubsection{Resulting Co Deposition on $\mathrm{Au}(111)$}

The electrolyte solution for electrochemical deposition of $\mathrm{Co}$ on $\mathrm{Au}(111)$ is composed of $0.1 \mathrm{M}$ sodium sulfate $\left(\mathrm{Na}_{2} \mathrm{SO}_{4}\right), 0.5 \mathrm{mM}$ sulfuric acid $\mathrm{H}_{2} \mathrm{SO}_{4}(\mathrm{pH} \approx 3)$, and $1 \mathrm{mM}$ cobalt sulfate $\mathrm{CoSO}_{4}$, which has been discussed in detail in Section 2.4.1 above. To minimize undesired reactions, the electrolyte is deaerated in-situ by $5 \mathrm{~N}$ argon gas prior to all investigations as demonstrated in Section 2.3.2 above. In addition, the electrochemical cell is hermetically sealed, as illustrated in Figure 2.4(b), to prevent a contamination with oxygen over time.

$\mathrm{CV}$ investigations of $\mathrm{Au}(111)$ in such an electrolyte solution are shown in Figure 2.13(a), without (gray) and with $1 \mathrm{mM} \mathrm{CoSO}_{4}$ (black) in solution. The calculated Nernst potential is $E_{0}\left(C o / \mathrm{Co}^{2+}\right)=-365 \mathrm{mV}$ for a $1 \mathrm{mM} \mathrm{CoSO} 4$ solution, according to Equation (2.5). The cathodic current densities in both CVs increase with decreasing potential $E_{\mathrm{WE}}$. In the $\mathrm{CV}$ with added $\mathrm{CoSO}_{4}$ (black) Co deposition is reflected by the steep increase of the cathodic current density (A). Since the hydrogen evolution is a competing reaction, a higher overpotential for the Co deposition is required: $E_{\text {Co depo }}<E_{0}\left(C o / C o^{2+}\right)=-365 \mathrm{mV}$. In upwards direction the Co dissolution is given by the corresponding anodic peak A' around $-190 \mathrm{mV}$, identified in Figure 2.13(a). The measured CVs are in good agreement with literature [102].

By use of a semi-logarithmic Tafel plot as shown in Figure 2.13(b) the onset of the hydrogen evolution and of the Co deposition are easily identified by linear fits (dashed lines) of the cathodic current densities $j_{\text {cat }}$. Caused by the hydrogen evolution both CVs (without and with $\mathrm{CoSO}_{4}$ ) show an increasing cathodic current below $E_{\mathrm{WE}} \approx-300 \mathrm{mV}$. By this presentation, the deposition of Co is fitted to occur below $-490 \mathrm{mV}$. Additionally, the further decrease in the cathodic current densities is caused by a depletion of $\mathrm{H}^{+}$and $\mathrm{Co}^{+}$cations at the $\mathrm{Au}(111)$ working electrode, highlighted by the gray square in Figure 2.13(b).

Within a potential range from $-450 \mathrm{mV}$ to $-330 \mathrm{mV}$ no Co is deposited on $\mathrm{Au}(111)$ and already deposited Co remains on the $\mathrm{Au}(111)$ surface. Therefore, the substrate potential $E_{\mathrm{WE}}$ is adjusted within this potential range for subsequent STM measurements. Thereby, stable electrochemical conditions for metal Co on $\mathrm{Au}(111)$ are ensured.

The low concentration of $\mathrm{H}_{2} \mathrm{SO}_{4}$ and $\mathrm{CoSO}_{4}$ causes a depletion of $\mathrm{H}^{+}$and $\mathrm{Co}^{2+}$ ions on the working electrode below $-510 \mathrm{mV}$, since the flux of the reactants depends 

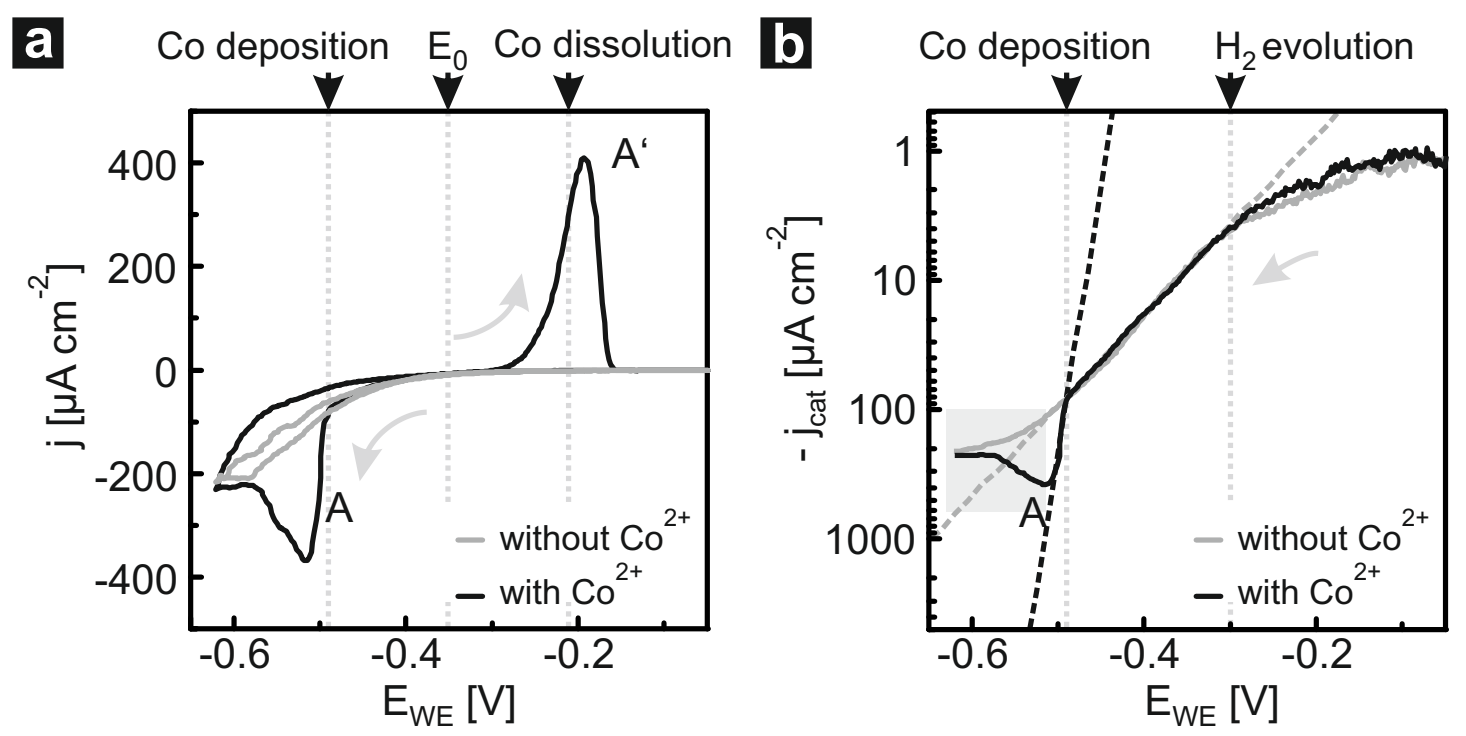

Figure 2.13: (a) CVs with $\frac{d E_{\mathrm{WE}}}{d t}=10 \mathrm{mV} \mathrm{s}^{-1}$ of $\mathrm{Au}(111)$ in $0.1 \mathrm{M} \mathrm{Na}_{2} \mathrm{SO}_{4}$ with $0.5 \mathrm{mM}$ $\mathrm{H}_{2} \mathrm{SO}_{4}$ (gray), and upon adding $1 \mathrm{mM} \mathrm{CoSO}_{4}$ (black). $E_{0}\left(\mathrm{Co} / \mathrm{Co}^{2+}\right)=-365 \mathrm{mV}$ for a $1 \mathrm{mM} \mathrm{CoSO}_{4}$ solution. Current densities of both CVs increase with decreasing potential caused by hydrogen evolution. The Co OPD is reflected by a steep increase of the cathodic current density (A). The corresponding Co dissolution is given by the anodic peak A' around $-190 \mathrm{mV}$. (b) A Tafel plot of the cathodic current density $j_{\text {cat }}$ of both CVs. Due to the semi-logarithmic scale the $\mathrm{H}_{2}$ evolution as well as the Co deposition can be linearly fitted (dashed lines). Thus, the onset of the hydrogen evolution is found below $-300 \mathrm{mV}$ and the Co deposition is observed below $-490 \mathrm{mV}$.

Depletion of $\mathrm{H}^{+}$and $\mathrm{Co}^{+2}$ cations is highlighted by the gray square.

on diffusion. Therefore, hydrogen evolution as well as Co deposition decrease at a large overpotential. This effect decelerates Co deposition at the $\mathrm{Au}(111)$ working electrode. Since Co deposition on $\mathrm{Au}(111)$ is an OPD depending strongly on the adjusted potential $E_{\mathrm{WE}}$, and since only a low Co coverage is desired, there is no reason to use a $\mathrm{CoSO}_{4}$ solution of higher concentration. A higher concentration of $\mathrm{H}_{2} \mathrm{SO}_{4}$ would suppress the $\mathrm{Co}$ deposition due to the strongly competing nature of the reaction. For these reasons the concentration of $\mathrm{H}_{2} \mathrm{SO}_{4}$ and $\mathrm{CoSO}_{4}$ in the electrolyte solution are set to $0.5 \mathrm{mM}$ and $1 \mathrm{mM}$, respectively.

\subsection{Conclusion}

The electrochemical cell in use is airtight and the electrolyte solution was deaerated in-situ by argon gas. Since only cleaned components as well as aqueous solutions of high purity were utilized, a clean environment was ensured for investigations 


\section{ELECTROCHEMISTRY}

at the solid/liquid interface. Therefore, no appreciable contamination or dissolved oxygen as observable in $\mathrm{CVs}$ of $\mathrm{Au}(111)$ in sodium sulfate $\left(\mathrm{Na}_{2} \mathrm{SO}_{4}\right)$ and sulfuric acid $\left(\mathrm{H}_{2} \mathrm{SO}_{4}\right)$.

Cobalt deposition on $\mathrm{Au}(111)$ occurred below the calculated Nernst potential since it is an OPD, depending on the actual concentration of the $\mathrm{Co}^{2+}$ cation in solution. Caused by a required deposition potential in the range of $E_{\mathrm{WE}}=-490 \mathrm{mV}$ hydrogen evolution occurred at the $\mathrm{Au}(111)$ working electrode as well. The hydrogen evolution was strongly reduced by use of sodium sulfate $\left(\mathrm{Na}_{2} \mathrm{SO}_{4}\right)$ as supporting electrolyte solution instead of sulfuric acid $\left(\mathrm{H}_{2} \mathrm{SO}_{4}\right)$. In addition, deaeration of the aqueous solution avoided undesired reaction with oxygen, and thus metal deposition in the lower potential range could be achieved without any disturbances.

However, Co red/ox reactions depend on the actual $\mathrm{pH}$-value at the working electrode. Stirring of the electrolyte solution is not possible for STM investigations at the solid/liquid interface performed during Co deposition. Therefore, a depletion of $\mathrm{H}^{+}$cations arose at the $\mathrm{Au}(111)$ working electrode at low potentials. The diffusion controlled mass transport of $\mathrm{H}^{+}$cations in differing electrolyte solutions (I-III) was observed by potential step measurements, which simulated a sample preparation during STM measurements. Thereby, a depletion of $\mathrm{H}^{+}$cations at the working electrode led to a diffusion induced shift of the $\mathrm{pH}$-value $\left(-\log \left(C_{H^{+}}\right)\right)$into the alkaline range. This caused the formation of cobalt complexes near the working electrode, which was electrochemically observed by changes over time in CVs of $\mathrm{Au}(111)$ in a solution composed of sodium sulfate $\left(\mathrm{Na}_{2} \mathrm{SO}_{4}\right)$ and cobalt sulfate $\left(\mathrm{CoSO}_{4}\right)$.

As a result, a small amount of sulfuric acid $\left(\mathrm{H}_{2} \mathrm{SO}_{4}\right)$ has to be added to the electrolyte solution causing $p H \approx 3$ to avoid undesired secondary reactions of deposited $\mathrm{Co}$ on $\mathrm{Au}(111)$. This way, an electrochemical deposition of metal $\mathrm{Co}$ on $\mathrm{Au}(111)$ is accomplished and the Co remains stable on $\mathrm{Au}(111)$ in a potential range from $-450 \mathrm{mV}<E_{\mathrm{WE}}<-330 \mathrm{mV}$. 


\section{Chapter 3}

\section{Scanning Tunneling Microscopy}

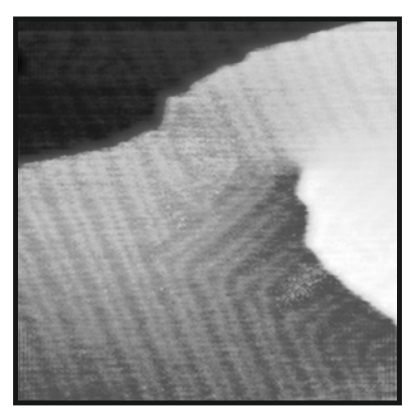

Scanning tunneling microscopy (STM) was first realized by Binnig et al. in 1981 [15, 16, 17, 18, 19]. A general theoretical formalism for electrons tunneling across a barrier was made by Bardeen [10] in 1961, which was used by Tersoff and Hamann to describe electrons tunneling from the STM tip to the investigated substrate [189, 190]. The development of STM has constituted a milestone which enabled investigations of surfaces at the atomic scale. In contrary to other surface characterization techniques which require electron detection, STM is not confined to ultra high vacuum (UHV) conditions and has been realized under ambient and higher pressures for studies in solid/gas and solid/liquid interfaces 63. Thereby, it is a powerful tool to investigate in-situ electrochemical processes and structure formations of adlayers at the solid/liquid interface with high resolution [41, 43, 44, 118. Growth processes during deposition at the solid/liquid interface often differs from deposition in UHV and STM allows in-situ investigations with high spatial resolution.

This chapter includes a short introduction of the theory and the experimental setup of STM in Section 3.1 (for more detailed descriptions compare [39, 197]).

Furthermore, the tunneling process at the solid/liquid interface is discussed and compared to the tunneling process at the solid/vacuum interface in Section 3.2 . In Section 3.2 metal growth on the working electrode in the electrochemical environment is demonstrated by Co deposition on $\mathrm{Au}(111)$ and investigated in-situ by STM. The Co dissolution process is observed in-situ by STM in addition to cyclic voltammetry measurements. 


\subsection{STM - Theoretical Approach}

STM is the investigation of a surface with a sharp conducting tip utilizing tunneling electrons.

The formula of the tunneling current in one dimension between two planar electrodes was described perturbatively by Bardeen [10], and the formalism was adapted by Tersoff and Hamann [189] [190] [188] to match the experimental conditions of STM, i.e. exchanging one planar electrode by the tip with a spherical apex.

In Bardeen's formalism the tunneling current $I_{0}$ between two electrodes $\mu$ and $\nu$ in the first order is given by

$$
I_{0}=\frac{2 \pi e}{\hbar} \sum_{\mu \nu} f\left(E_{\mu}\right)\left[1-f\left(E_{\nu}+e V_{b}\right)\right]\left|M_{\mu \nu}\right|^{2} \delta\left(E_{\mu}-E_{\nu}\right)
$$

where $\hbar$ is the reduced Planck constant, $e$ is the elementary electric charge, $V_{b}$ is the bias voltage, $f(E)$ is the Fermi function, $M_{\mu \nu}$ is the tunneling matrix element between states $\psi_{\mu}$ of $\mu$ and $\psi_{\nu}$ of $\nu$ at the energy $E_{\mu, \nu}$ [190]. Thereby, the tunneling matrix element connects the states of both electrodes, describing an overlap of the two wavefunctions when integrating over the separating area [189]

$$
M_{\mu \nu}=\frac{-\hbar^{2}}{2 m} \int d \vec{S} \cdot\left(\psi_{\mu}^{*} \nabla \psi_{\nu}-\psi_{\nu} \nabla \psi_{\mu}^{*}\right)
$$

In the limits of small voltages and room temperature Equation 3.2 can be simplified by

$$
I_{0}=\frac{2 \pi e^{2}}{\hbar} V_{b} \sum_{\mu \nu}\left|M_{\mu \nu}\right|^{2} \delta\left(E_{\nu}-E_{F}\right) \delta\left(E_{\mu}-E_{f}\right)=\frac{2 \pi e^{2}}{\hbar} V_{b} \rho_{\text {tip }}\left(E_{F}\right) \rho_{\text {sub }}\left(E_{F}\right),
$$

with the Fermi function approximated by a step function [190].

Reducing electrode $\mu$ to a tip with a single atom in its apex, approximated by a s-wave tip function, the matrix element is proportional to the amplitude of $\psi_{\nu}$ at the position $\overrightarrow{r_{0}}$ [190]

$$
\begin{aligned}
& I_{0} \propto V_{b} \exp [2 \kappa R] \rho_{\text {tip }}\left(E_{F}\right) \sum_{\nu}\left|\psi_{\nu}\left(\overrightarrow{r_{0}}\right)\right|^{2} \delta\left(E_{\nu}-E_{F}\right) \\
& I_{0} \propto V_{b} \exp [2 \kappa R] \rho_{\text {tip }}\left(E_{F}\right) \rho_{\text {sub }}\left(\overrightarrow{r_{0}} ; E_{F}\right),
\end{aligned}
$$

where $\rho_{\text {sub }}\left(\overrightarrow{r_{0}} ; E_{F}\right)=\sum_{\nu}\left|\psi_{\nu}\left(\overrightarrow{r_{0}}\right)\right|^{2} \delta\left(E_{\nu}-E_{F}\right)$ is the surface local density of states (LDOS) at the Fermi level and at the point $\overrightarrow{r_{0}}$, which is the vector defined by the center of curvature of the effective tip with a radius $\mathrm{R}$ and the closest point to substrate electrode $\nu$ [190]. $\quad \kappa=\frac{\sqrt{2 m \phi}}{\hbar^{2}}=\left(5.1 \mathrm{eV}^{-0.5} \mathrm{~nm}^{-1} \sqrt{\phi}\right)$ is the decay 
constant in the potential barrier region, with the electron mass $m$ and the local potential barrier height $\phi$.

The wave function $\nu$ decays exponentially with the distance $\mathrm{z}$ from the electrode surface,

$$
\begin{aligned}
\psi_{\nu}(\vec{r}) & \propto \exp [-\kappa z] \\
\rightarrow\left|\psi_{\nu}\left(\overrightarrow{r_{0}}\right)\right|^{2} & \propto \exp [-2 \kappa(d+R)]
\end{aligned}
$$

and thus the tunneling current, Equation 3.5, depends exponentially on the distance $d$ between the substrate (electrode $\nu$ ) and the tip apex.

$$
I \propto V_{b} \exp [-2 \kappa \mathrm{d}] \rho_{t i p}\left(E_{F}\right) \rho_{s u b}\left(\overrightarrow{r_{0}} ; E_{F}\right)
$$

The STM setup employed in this work consists of four components: a conductive substrate, a probing metallic tip pointing to the substrate, a piezo-ceramic element that is capable of positioning the tip apex with respect to the substrate surface in three dimensions, and the electronic control unit.

The tip is mounted onto the piezoelectric ceramic tube 1 , which is deflected proportionally to the applied voltages $V_{x}, V_{y}$, and $V_{z}$. A certain surface area of the investigated substrate is mapped by moving the tip in the direction of the x-axis (fast scanning axis) back and forth, and subsequently moving in the direction of the $\mathrm{y}$-axis (slow scanning axis) and repeating the $\mathrm{x}$-axis scan. The scanning tube in use has a lateral operating limit of close a square area of $12 \mu \mathrm{m}$.

Depending on the distance $d$ and the bias voltage $V_{b}$ between the tip apex and the substrate, a tunneling current $I_{0}$ is established:

$$
I_{0} \propto V_{b} \exp [-2 \kappa \mathrm{d}]=V_{b} \exp \left[-10.2 \mathrm{eV}^{-0.5} \mathrm{~nm}^{-1} \sqrt{\phi} \mathrm{d}\right],
$$

where $d$ is given in nm and $\phi$ in eV. For STM in vacuum $\phi$ is equal to the work function of the material in use with a typical value of approximately $5 \mathrm{eV}$ for metals. A reduction of the tip-surface distance $d$ leads to an overlap of the wave functions of the two electrodes. There, electrons from occupied states of one electrode can tunnel into unoccupied states of the other. By applying a bias voltage $V_{b}$ between tip and substrate a net tunneling current can be detected.

The STM setup operates in two possible modes: the constant-current mode (CCM) and the constant-height mode (CHM), illustrated schematically in Figure 3.1 (a) and (b), respectively.

In CCM a setpoint value is given for the tunneling current $I_{0}$. Constant current scanning is achieved by a STM feedback loop of the tunneling current which adjusts

\footnotetext{
${ }^{1}$ PI Ceramic
} 

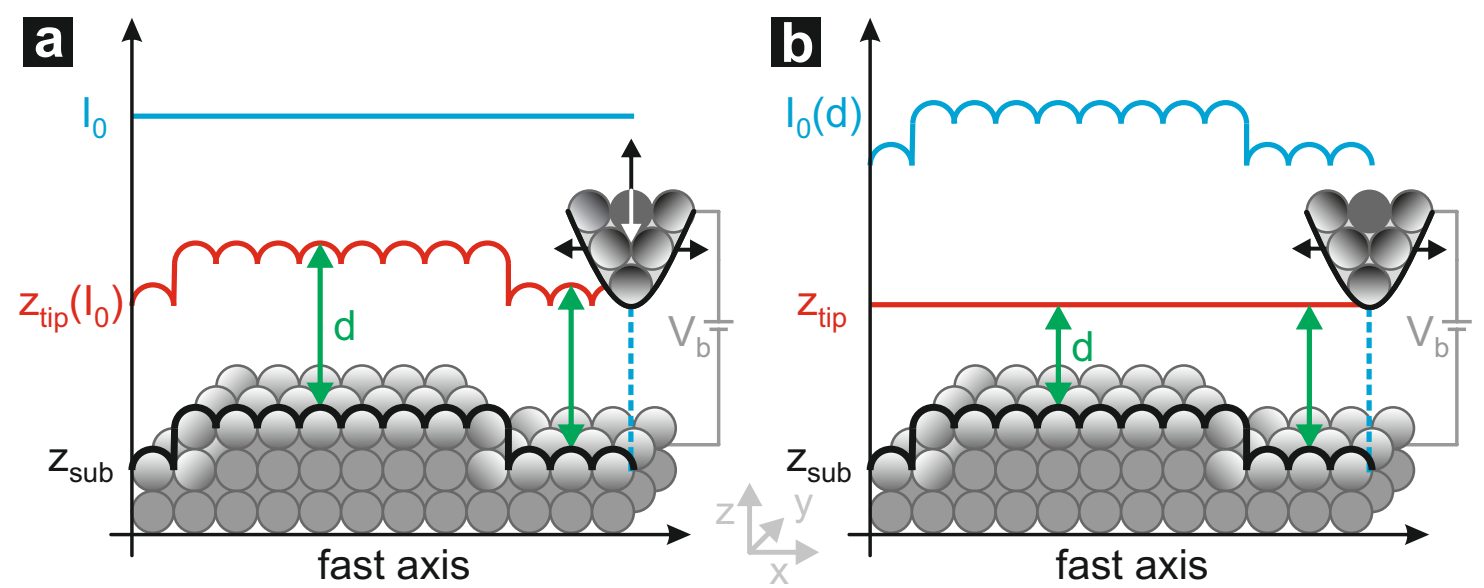

Figure 3.1: Schematic illustration of the STM scanning modes: (a) constant-current mode, where the tunneling current is set to $I_{0}$, and is kept constant during measurement by keeping $d=\left|z_{\text {tip }}-z_{\text {sub }}\right|$ constant. The height of the tip $z_{\text {tip }}$ has to be adjusted by a piezoelectric ceramic tube. Topographical information of the probed surface are delivered by the changing tip height. (b) constant-height mode, where the tip height $z_{\text {tip }}$ is kept constant. Caused by the topography of the substrate $z_{\text {sub }}$ the tip-surface distance varies $d$. The change in tunneling current $I_{0}(d)$ delivers topographical information of the probed substrate.

$d=\left|z_{\text {tip }}-z_{\text {sub }}\right|$ accordingly. The tip-surface separation $d$ along the z-axis plotted as a function of $x$ and $y$ represents the mapping of the constant surface LDOS at $V_{b}$ and corresponds to the real topography assuming for constant surface states.

In CHM, the scanning plane of the tip apex is fixed. Consequently the tip-surface distance $d=\left|z_{\text {tip }}-z_{\text {sub }}\right|$ is changing due to the topography of the surface. The resulting tunneling current $I_{0}(d)$ delivers topographical information of the probed substrate. Without the need of the feedback loop and subsequent adjustment of the tip vertical position the STM can scan faster, but also with significant risk of a physical overlap of the tip and substrate, due to the corrugation of latter.

In the present work all STM investigations were performed in CCM, also in order to realize further spin-polarized STM measurements.

\subsection{STM in the Electrochemical Environment}

In this section the electrochemical STM (ec-STM) setup for investigations at the solid/liquid interface is laid out. A detailed description of the tip preparation is given followed by an example of ec-STM measurements on a reconstructed $\mathrm{Au}(111)$ surface. 


\subsubsection{Setup of the Ec-STM}

STM at the solid/liquid interface was initially realized by Sonnenfeld and Hansma in a two-electrode arrangement, specifically a STM tip and substrate [50, 177, 178]. Control over electrochemical reactions, and thereby improvement of the stability of the substrate and the tip, was first accomplished by a four-electrode arrangement [95, [15, 196]. The potential adjustment of each working electrode ( $W E_{\text {tip }}$ and $W E_{\text {sub }}$ ) was achieved by a separate potentiostat (bipotentiostat) with help of a reference electrode $(R E)$ and a counter electrode $(C E)$ [106, 107]. Such an electrochemical cell under the control of a bipotentiostat was already introduced and illustrated in Chapter $2{ }^{1}$. A sketch of the ec-STM setup with all control units and its adjustment of the tip by a piezoelectric ceramic tube is depicted in Figure 3.2. With the STM setup integrated into an electrochemical cell, in-situ investigations enable the direct observation of surfaces and electrochemical processes at the solid/liquid interface on the nanometre scale [71] [41].

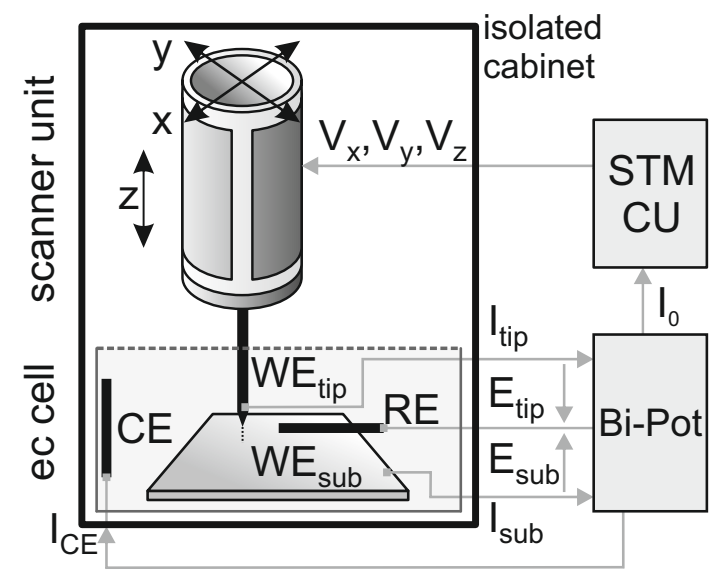

Figure 3.2: Setup of the ec-STM, which is a combination of an electrochemical cell with four electrodes (substrate working electrode $W E_{\text {sub }}$, tip working electrode $W E_{\text {tip }}$, reference electrode $R E$, and counter electrode $C E$, controlled by the bipotentiostat) and a STM setup. The STM control unit (CU) can adjust the tip position in all three dimensions by applying voltages $V_{x}, V_{y}$, and $V_{z}$ to the piezoelectric ceramic tube.

The electrochemical cell and the STM scanner are positioned on a heavy platform which is hanging form the ceiling by four steel springs, in order to decouple vibrationally the instrument from the building. Additional isolation from acoustic vibrations is provided by foam which surrounds the instrument housing (isolated cabinet in Figure 3.2 and its entrance. The electronics and operating PC are located outside of this isolated cabinet.

In an electrochemical environment the potential of the tip $E_{\text {tip }}$ as well as the potential of the substrate $E_{\mathrm{WE}}$ are controlled with respect to the reference electrode $R E$ by an appropriate bipotentiostat [41, 115]. Consequently, the bias voltage of the STM setup $V_{\mathrm{b}}$ is given by the difference between the potentials of both working electrodes $V_{\mathrm{b}}=\left|E_{\mathrm{tip}}-E_{\mathrm{WE}}\right|$. The in-situ preparation of the samples can be achieved

\footnotetext{
${ }^{1}$ The electrochemical STM used in this work consists of a NanoScope E control unit with a bipotentiostat BP-700 from EC-Tec.
} 
via adjusting the potential of the working electrode, such as depositing solved cations out of solution and subsequent dissolution. These electrochemical processes has been introduced in Chapter 2. Hence, for ec-STM no heating and sputtering cycles are needed for in-situ surface preparation.

Distance-tunneling spectroscopy, where the tunneling current is measured as a function of the tip-surface distance $d$, in vacuum results in spectra of exponential functions. By such measurements the tunnel barrier height (work function) $\phi_{M}$ of the investigated material $M$ is given by the gradient of the semi-logarithmic plot of

$$
\phi_{M} \approx \frac{1}{A^{2}}\left(\frac{d \ln (I)}{d \mathrm{~d}}\right)^{2},
$$

with $A=\left(2 \times 5.1 \mathrm{eV}^{-0.5} \mathrm{~nm}^{-1}\right)=\left(10.2 \mathrm{eV}^{-0.5} \mathrm{~nm}^{-1}\right)$ according to Equation (3.9) [20, 60, 65, 66, 111, 207]. For example, in vacuum the $\mathrm{Co}(0001)$ work function $\phi_{\mathrm{Co}}$ is $\sim 5.264 \mathrm{eV}$ at $27^{\circ} \mathrm{C}\left[157\right.$, 158] and the $\mathrm{Au}$ work function $\phi_{\mathrm{Au}}$ is $\sim 5.38 \mathrm{eV}$ at $27^{\circ} \mathrm{C}$ [157, 158].

Tunneling in an electrochemical environment is comparable to tunneling in vacuum, however, the process is influenced by ordered adlayers of water molecules at the investigated surface [85, 90, 132, 133, 170]. Such a double-layer of water molecules at the solid/liquid interface modifies the exponential dependence measured by distancetunneling spectroscopy. As a result, the tunnel barrier height is about $1 \mathrm{eV}$ to $2 \mathrm{eV}$, lower than in vacuum [126]. According to Equation (3.10) in the electrochemical environment an effective reduced tunnel barrier height $\phi_{M \text {,eff }}<\phi_{M \text {,UHV }}$ results [14, 53, 75, 76, 77, 85, 142, 153, 159, 169, 176, 193.

Hugelmann and Schindler observed by distance-tunneling spectroscopy an oscillation of the tunneling current at the solid/liquid interface [92, 93]. Thereby, distance-tunneling spectroscopy was obtained by discretely changing the tip-surface separation of $\delta \mathrm{d} \approx 0.034 \mathrm{~nm}$ per step to exclude thermal effects [92, 93]. The tunneling current was measured over a range from $10^{-12} \mathrm{~A}$ to $10^{-4} \mathrm{~A}$ for a tip/sample bias voltage $V_{b}=100 \mathrm{mV}$. The subtraction of the mean gradient from the semilogarithmic curve of the work function reveals an oscillation with period of $0.35 \mathrm{~nm}$, corresponding to the dimension of a water molecule [13, 170]. The oscillation amplitude was measured to be $\pm 0.8 \mathrm{eV}$ around the mean tunnel barrier height $\phi_{\text {eff }}=1.51 \mathrm{eV}[93$. The effective reduced tunnel barrier height results from tunneling via intermediate states [77, 153] and calculations show that tunneling via hydrogen atoms results in a potential barrier in the range of measured values [13, 58, 170].

Thereby, it was shown that tunneling for distances $d<0.15 \mathrm{~nm}$ occurs across a vacuum-like gap where the barrier height increases with increasing tip-surface separation $d$, until the first adlayer of water molecules penetrates the opening between tip and substrate $(d>0.35 \mathrm{~nm})$. Thus, the barrier height is decreasing due to tunneling via hydrogen intermediate states [92, 93]. The same behavior is observed 
for the second adlayer of water molecules and the third up to a distance where the faradaic current, generated by reactions at the tip working electrode exposed to the electrolyte.

\subsubsection{Ec-STM Tips - Fabrication and Characterization}

In ec-STM tips have to be inert in the electrochemical environment at the adjusted potential. Thus, STM tips are often fabricated from noble metals such as Au, Pt or PtIr serving this purpose. The STM tips used in this work were made of $\mathrm{Au}, \mathrm{Ni}, \mathrm{Ni}$ coated $\mathrm{Au}$, or $\mathrm{Co}_{68.2} \mathrm{Fe}_{4.3} \mathrm{Si}_{12.5} \mathrm{~B}_{15}{ }^{1}$. However, the tip preparation of all mentioned materials is very similar and described in the following.

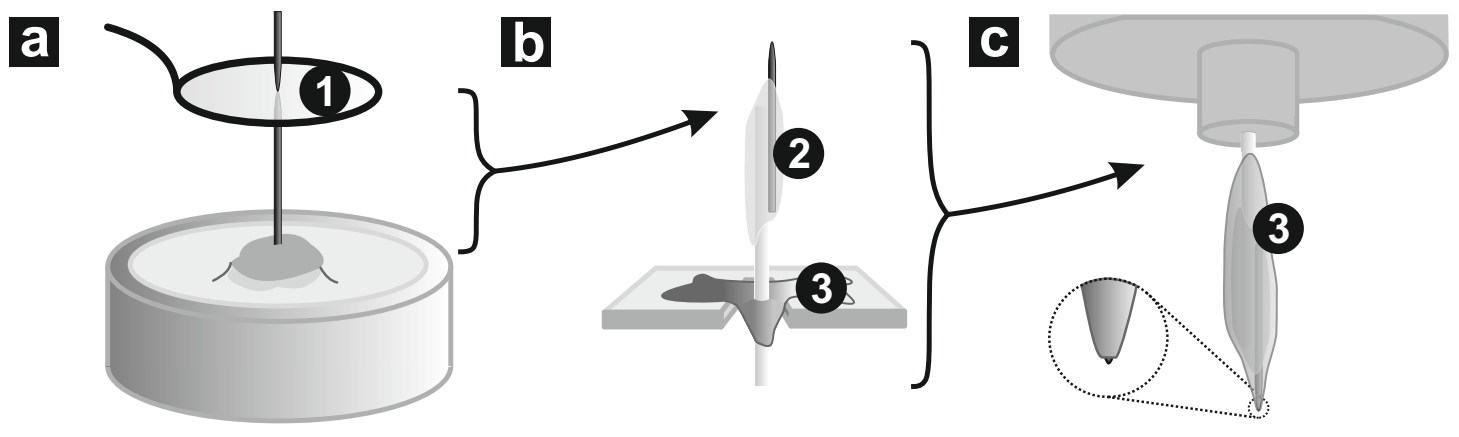

Figure 3.3: Tip preparation by drop-off technique: (a) Electrochemical etching of the tip wire within a lamella (1) of acid. After the etching process the tip drops down and is collected in pure water and cleaned from remaining acid. This sudden interruption ensured pointed tips. (b) Tips made of wires too thin for the ec-STM tip clamp were glued (2) to mechanically cut Au wire of the appropriate thickness. All tips were isolated by an Apiezon wax film (3) which exposed only the tip apex in order to reduce the faradaic current. (c) The STM tip clamped into the ec-STM setup (tip holder).

STM tips were electrochemically etched by a modified drop-off fabrication to achieve a sharp tip apex [27] [124] [36]. The tip preparation steps are illustrated in Figure 3.3 . First, the tip wire is led through a platinum wire ring electrode with a diameter of $10 \mathrm{~mm}$, where a lamella of an electrolyte is adhered, Figure 3.3(a). The ring electrode acts as a cathodic etching electrode, whereas the metal wire acts as an anodic electrode. For Au tip etching the electrolyte lamella consists of $32 \%$ hydrochloric acid [94. For Ni tips the electrolyte is composed of $10 \%$ hydrogen peroxide and $95 \%$ sulphuric acid at a ratio of 10:1 [57]. Due to an applied voltage between the ring electrode and the metal wire an electrochemical etching process occurs in the electrolyte plane.

\footnotetext{
${ }^{1} \mathrm{Co}_{68.2} \mathrm{Fe}_{4.3} \mathrm{Si}_{12.5} \mathrm{~B}_{15}$ is a soft ferromagnetic and amorphous alloy, which is often called AC20 in the following. Produced by UNITIKA using an 'In-Rotation-Water-Spinning Process' [137].
} 
Figure 3.4: Typical etching current $I_{e}$ over time $t$ is illustrated for an Au tip preparation. The arising etching current decreases until the lower part of the metal wire drops. The upper metal wire still remains in the acid lamella and causes a low residual etching current until the etching voltage is turned off.

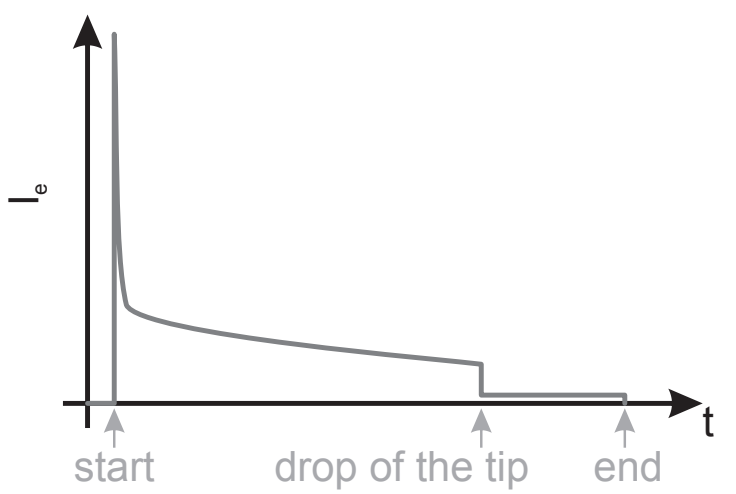

The etching voltages required for $\mathrm{Au}$ and $\mathrm{Ni}$ tips are $1.65 \mathrm{~V}$ and $1.3 \mathrm{~V}$, respectively. During the etching process the lamella should be stable without breaking to get an optimal result. Directly after the etching process, the lower part drops off and is collected in the water reservoir underneath. For very thin and therefore very light wires, a weight is attached to the lower part of the tip, so as to overcome the surface tension of the lamella. By the drop of the tip, the etching process does not continue after the tip formation resulting in sharp and stable tips. The etching voltage was optimized by examining the outcome of the etching process by scanning electron microscopy (SEM). A SEM image on a typical electrochemically etched Au tip is shown in Figure 3.5 .

Figure 3.5: SEM images of an Au tip directly after electrochemical etching with (a) $150 \times$ magnification and (b) $90000 \mathrm{x}$ magnification. The etched area of the wire depends on the thickness of the electrolyte lamella. A regular and pointed tip is prepared by placement in the center of the lamella

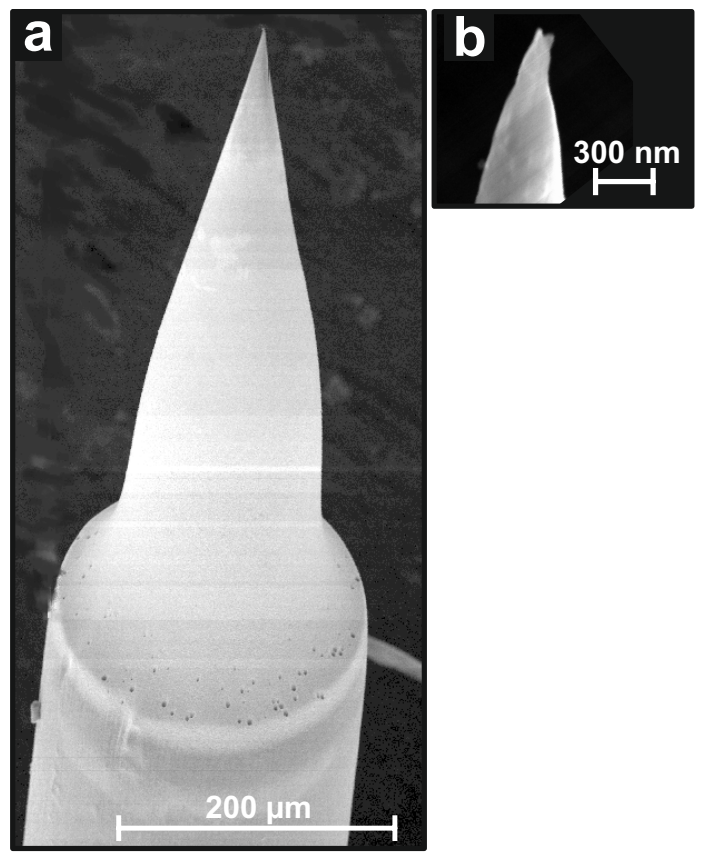

The STM setup allows mounting of tips with a diameter of $250 \mu \mathrm{m}$ (tip holder), 
therefore tips with smaller diameters have to be glued to an $\mathrm{Au}$ wire carrier, Figure 3.3(b), by conductive silver paint. Finally, all tips used in the electrochemical environment are protected by an Apiezon wax film, where only the tip apex is left bare for $\approx 10 \mathrm{\mu m}^{2}$ to enable a tunneling contact between tip and substrate [64, 164]. Therefore, Apiezon wax is heated and melted by a soldering iron with a notch where the tip is passed through [131]. This preparation step is monitored via an optical microscope. The isolation is needed to reduce the faradaic current generated by reactions at the tip working electrode exposed to the electrolyte. Otherwise the tunneling current is marginal compared to the faradaic current [9] [39] and ec-STM is not possible.

After these preparation steps, the finished STM tip is clamped in the scanning unit of the STM setup (Figure 3.3(c)) at the end of the piezoelectric ceramic tube.

\subsubsection{STM of $\mathrm{Au}(111)$ at the Solid/Liquid Interface}

STM measurements at the solid/liquid interface were performed on an $\mathrm{Au}(111)$ surface. The Au single crystal was is thermally treated in a hydrogen flame and cooled down in a nitrogen atmosphere prior to all investigations in order to prepare extended atomic terraces of $\mathrm{Au}(111)$. The atomic terraces of the $\mathrm{Au}(111)$ surfaces investigated exhibited the characteristic $\sqrt{3} \times 22$ reconstruction, known as herringbone reconstruction [11, 104, 105].

Once the substrate is in contact with the electrolyte solution, the potential of the substrate $E_{\mathrm{WE}}$ has to be adjusted below $400 \mathrm{mV}$, since at higher potentials the herringbone reconstruction is lifted due to the adsorption of $\mathrm{SO}_{4}^{2-}$ anions on $\mathrm{Au}(111)$, regarding the $\mathrm{CV}$ investigations of $\mathrm{Au}(111)$ shown in Figure 2.7 [51, 104, 120]. In this case, a plane $(1 \times 1)$ structured $\mathrm{Au}(111)$ surface would be observed until at higher potentials a $\sqrt{3} \times \sqrt{7}$ ordered adlayer of $\mathrm{SO}_{4}^{2-}$ anions is formed [43, 51, 104, 117, 120]. A nearly oxygen free electrolyte solution is ensured by in-situ deaeration of the ecSTM setup prior to all investigations, as described in 2.3.2.

Samples prepared with this procedure have $\mathrm{Au}(111)$ terraces extended over several hundred nanometers, as shown in Figure 3.6(a). The performance of the electrochemical STM is demonstrated by the observation of the $\sqrt{3} \times 22$ reconstructed $\mathrm{Au}(111)$ surface in the STM image 3.6(a) and in detail in Figure 3.6(b). This measurements reveals the herringbone reconstruction of the $\mathrm{Au}(111)$ surface on the extended terraces. A line-scan across five $\mathrm{Au}(111)$ step edges is plotted in Figure 3.6(c). The apparent height derived by the STM scanner motion in $\mathrm{z}$ is calibrated against the step height of a single $\mathrm{Au}(111)$ atomic layer $1 \mathrm{AL} \widehat{=} 2.4 \AA$ [11, 31] 1]. Therefore, the high resolution of the ec-STM is demonstrated in all three dimensions.

\footnotetext{
1 The uncertainty of each measured value $v$ is given by the standard deviation.
} 
Figure 3.6: Observation of a $\sqrt{3} \times 22$ reconstructed $\mathrm{Au}(111)$ surface by ecSTM at the solid/liquid interface in constant-current mode. Typical setpoint are: tunneling current $I_{0}=$ $10 \mathrm{nA}, \mathrm{Au}(111)$ substrate potential $E_{\mathrm{WE}}=-170 \mathrm{mV}, \mathrm{Au}$ tip potential $E_{\text {tip }}=80 \mathrm{mV}$, resulting in a bias voltage of $V_{b}=250 \mathrm{mV}$. (a) On a large scale the herringbone reconstruction is observed on wide terraces of the $\mathrm{Au}(111)$ surface and (b) can be investigated in detail. (c) The line profile of the line indicated in (a) shows monatomic steps with $(2.4 \pm 0.2) \AA$ height. Hence, the high resolution of the ec-STM is demonstrated in all three dimensions.

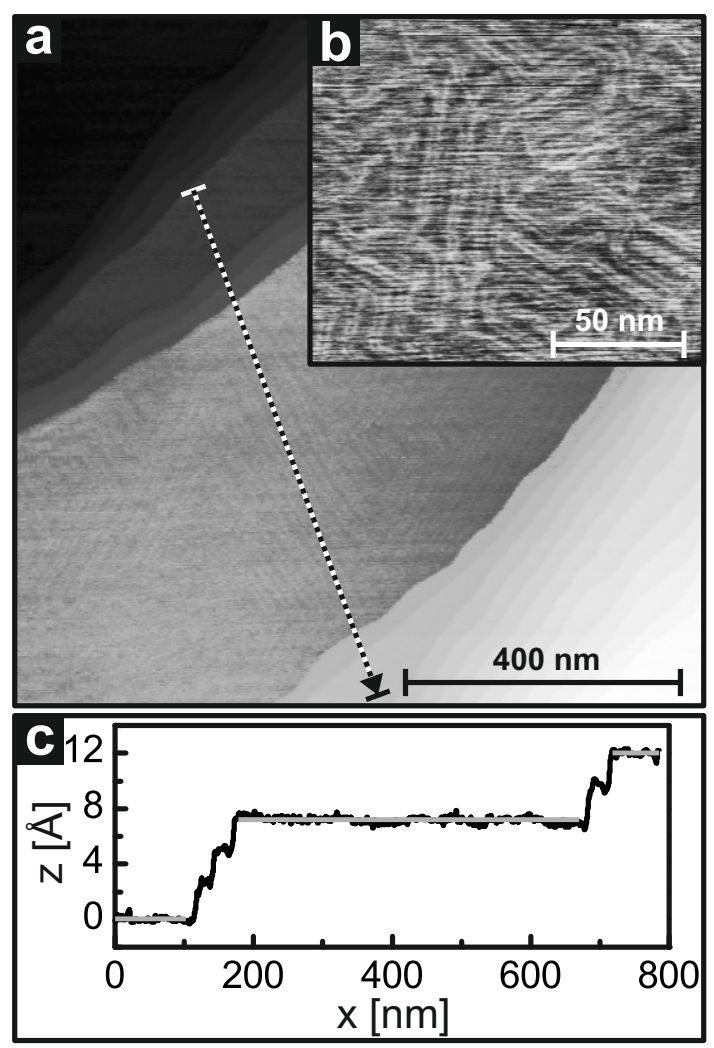

\subsection{STM of Co/Au(111) at the Solid/Liquid Interface}

The aqueous electrolyte for Co deposition on $\mathrm{Au}(111)$ consist of $0.1 \mathrm{M} \mathrm{Na}_{2} \mathrm{SO}_{4}$ and $1 \mathrm{mM} \mathrm{CoSO}_{4}$ with added $\mathrm{H}_{2} \mathrm{SO}_{4}$ to adjust the electrolyte solution to $p H \approx 3$, as already laid out in Section 2.4.1.

As mentioned above, once the ec-STM setup is prepared for experiments, the $\mathrm{Au}(111)$ substrate potential $E_{\mathrm{WE}}$ is adjusted to $30 \mathrm{mV}$. Initially, the electrolyte solution consists just of the supporting electrolyte solution $\left(\mathrm{Na}_{2} \mathrm{SO}_{4}\right.$ and $\left.\mathrm{H}_{2} \mathrm{SO}_{4}\right)$ to prevent an unintended Co deposition caused by higher concentration $C_{\mathrm{Co}^{2+}}$ or the formation of Co hydroxides, see Figure 2.12. Just before the completion of the insitu deaeration of the electrolyte, solved $\mathrm{CoSO}_{4}$ is added via the deaeration column, see Figure 2.4(b), in order to avoid a high concentration of $\mathrm{Co}^{2+}$ cations at the $\mathrm{Au}(111)$ substrate.

In the following the electrochemical deposition of Co onto a freshly prepared $\mathrm{Au}(111)$ surface is investigated by ec-STM. Furthermore the related $\mathrm{Co} / \mathrm{Au}$ interface is described in detail and the morphology of the electrochemically deposited Co on $\mathrm{Au}(111)$ is characterized. 


\subsubsection{Ec-STM Investigations}

For Co deposition the potential of the Au working electrode $E_{\mathrm{WE}}$ is adjusted from $30 \mathrm{mV}$ to $-470 \mathrm{mV}$, a value below the Nernst potential $\mathrm{E}_{0}\left(\mathrm{Co}_{\mathrm{C}} \mathrm{Co}^{2+}\right)$, according to $\mathrm{CV}$ investigations as shown in Figure 2.13. In order to observe the formation of Co layers, the line-scan frequency was increased to $f_{\mathrm{STM}} \approx 1.4 \mathrm{~Hz}$. Thereby, the Co deposition can be monitored in-situ and in real time. The low potential difference between tip $E_{\text {tip }}$ and substrate $E_{\mathrm{WE}}$ enables the simultaneous Co deposition and in-situ STM [183, 209].

When the desired Co coverage is reached, the potential of the Au working electrode $E_{\mathrm{WE}}$ is adjusted at about $-370 \mathrm{mV}$ close to the Nernst potential $E_{0}\left(\mathrm{Co} / \mathrm{Co}^{2+}\right)$. Therefore, neither Co dissolution nor additional Co deposition occurs, as shown in the CV in Figure 2.13(a). Thereby, electrochemically stable and extended Co islands are ensured during subsequent ec-STM and further sp-STM measurements. Two exemplary STM topographies of the $\mathrm{Co} / \mathrm{Au}(111)$ surface are shown in Figures 3.7 and 3.8. On top of the bare $\mathrm{Au}(111)$ terraces, the typical herringbone structure of the $\sqrt{3} \times 22$ reconstructed $\mathrm{Au}$ surface is observed, which is evidenced in the inset in Figure 3.7(a).

The deposited Co first attaches to $\mathrm{Au}(111)$ step edges, from where extended Co islands nucleate. These islands are a bilayer of Co with lateral size of several hundred nanometers arrange, as observed in Figure 3.8(a). Instead of a complete coverage of the $\mathrm{Au}(111)$ substrate with a bilayer of $\mathrm{Co}$, additional Co nucleate on top of the bilayer, resulting in Co islands of three and four atomic layers (AL), as shown in Figure 3.8(a).

Electrochemically grown Co layers on the fcc $\mathrm{Au}(111)$ substrate show a hcp structure, with the (0001) plane observed on the surface [7, 33, 34, 99]. On top of the deposited Co islands of $2 \mathrm{AL}$ up to $4 \mathrm{AL}$ thickness a characteristic moiré structure is observed [3, 4, 33, 129, 181], as highlighted in Figure 3.7(a). The moiré structure results from a mismatch between the in-plane lattice constant of $\mathrm{Co}(0001)$ and $\mathrm{Au}(111)\left(a_{\mathrm{Co}}=2.51 \AA, a_{\mathrm{Au}}=2.88 \AA\right)$, with a mismatch of $\frac{a_{\mathrm{Co}}-a_{\mathrm{Au}}}{a_{\mathrm{Au}}}=13 \%$ 174.

This moiré structure evidences epitaxial growth of hcp Co(0001) on fcc $\mathrm{Au}(111)$ via electrochemical deposition. The resolution of both surface properties, the typical herringbone structure of the $\sqrt{3} \times 22$ reconstructed $\mathrm{Au}(111)$ surface as well as the moiré structure on Co, reveal the high resolution than can be achieved in ec-STM.

The electrochemical Co deposition on $\mathrm{Au}(111)$ employed here resulted in significantly different growth from previously reported deposition at the solid/liquid interface [2] [102]. In contrast to previous reports, here the evolution of Co multilayers is observed before a saturated bilayer is established. The extended Co islands are measured to reach a thickness up to $4 \mathrm{AL}$ and are separated by extended areas of bare $\mathrm{Au}(111)$. 


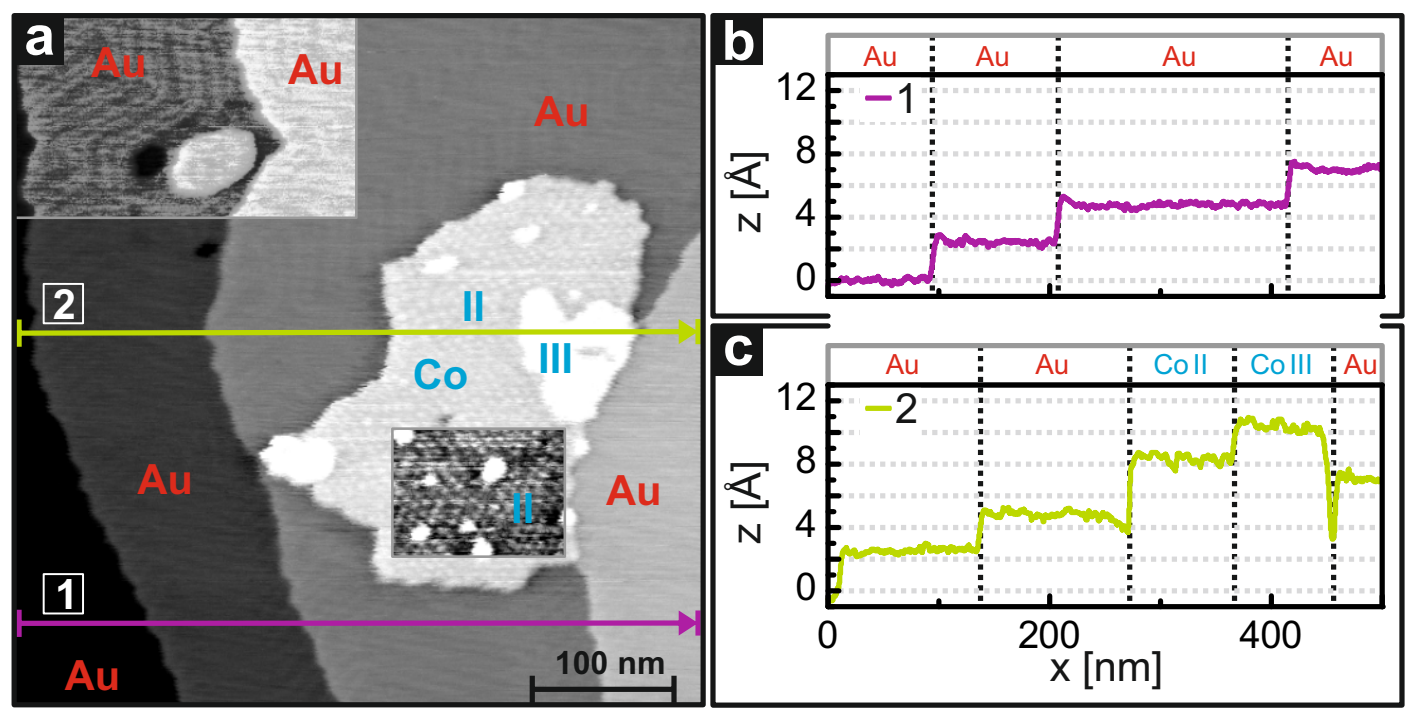

Figure 3.7: Topographical STM image of an electrodeposited Co island on Au(111) with the layer thickness indicated by Roman numerals. (a) $I_{0}=12 \mathrm{nA} ; E_{\mathrm{WE}}=-363 \mathrm{mV}$; $E_{\text {tip }}=-369 \mathrm{mV}$. The contrast was partially enhanced in image to highlight the $\sqrt{3} \times 22$ reconstructed $\mathrm{Au}(111)$ surface (upper left corner) as well as the moiré structure on top of the deposited Co island (lower part of the island) representing the high resolution of the ec-STM. Line profiles across the lines indicated in (a): (b) $1 \mathrm{AL} \mathrm{Au}$ step edge of $(2.4 \pm 0.3) \AA$, (c) 2 AL Co step edge of $(3.6 \pm 0.4) \AA$ with respect to the $\mathrm{Au}$ terrace below. On top of the bilayer Co island the third Co layer nucleates, which is about $(2.0 \pm 0.3) \AA$ high with respect to the underlying Co bilayer.

This type of growth seems to be attributed to the in-situ deaeration of the electrolyte with $5 \mathrm{~N}$ Ar gas. The differences between the CV measurement of the presented electrochemical setup and the ones reported in [2, 4, 34] reveal that the employed deaeration process suppresses interfering reactions on the $\mathrm{Au}(111)$ surface. In general, Co deposition at solid/liquid interfaces depends strongly on the supporting electrolyte and on the deposition potential at the $\mathrm{Au}(111)$ working electrode [33, 34].

In contrast to Co growth at the solid/liquid interface, in vacuum Co nucleation starts at the elbows of the herringbone reconstruction or randomly at the linearly reconstructed $\mathrm{Au}(111)$ surface [129, 194]. There, small Co islands are regularly arranged with $2 \mathrm{AL}$ in height [139, 140, 152, 173]. However, Co growth in vacuum strongly depends on the deposition temperature and even at larger nominal Co coverage the islands do not coalesce but increase vertically [129, 139, 150, 152, 191, 194. Since physical vapor deposition in vacuum differs significantly to the Co deposition in an electrochemical environment, a direct comparison between the properties of these $\mathrm{Co} / \mathrm{Au}(111)$ samples is not possible. 

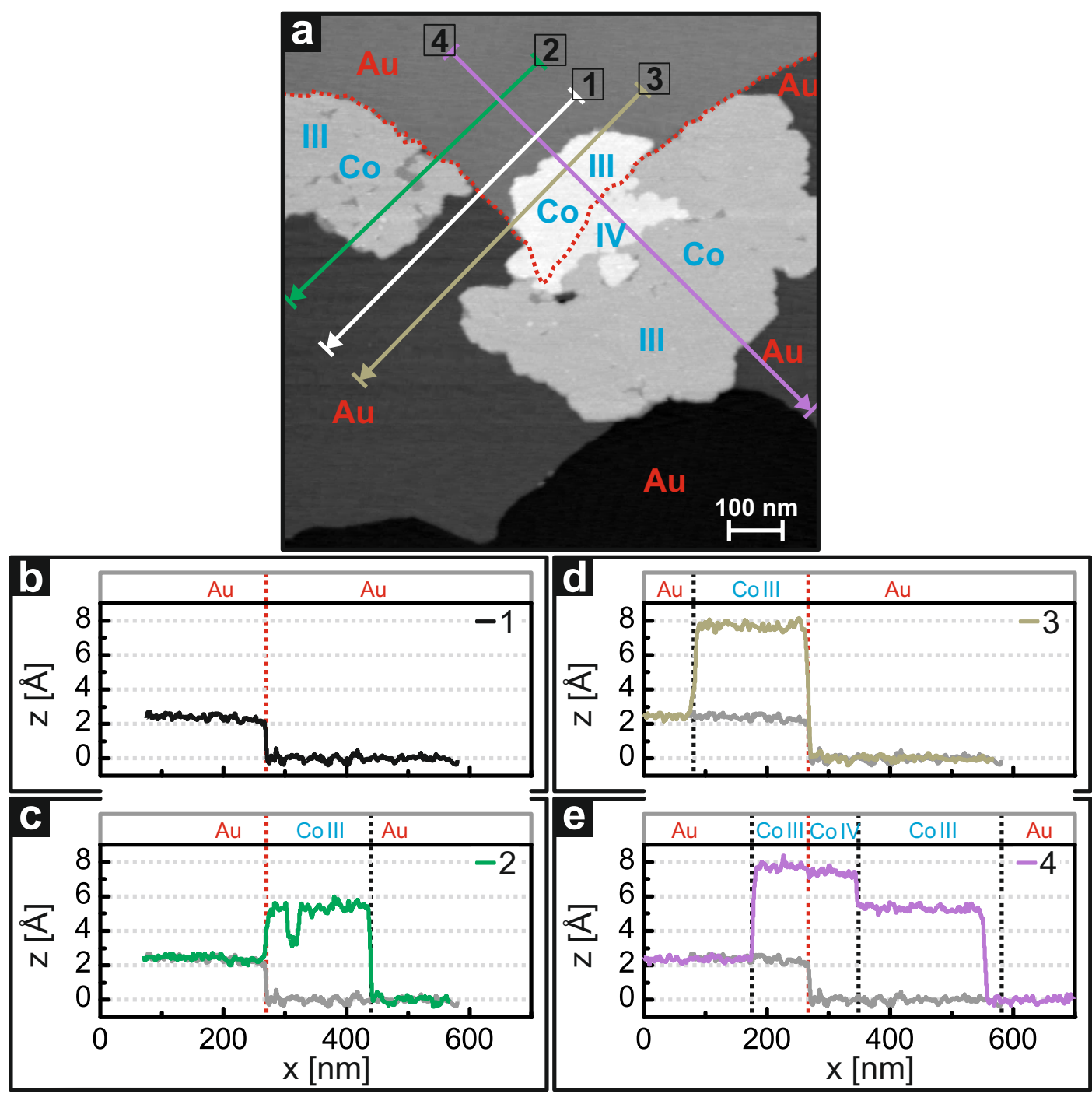

Figure 3.8: Topographical STM image of electrodeposited Co on Au(111): isolated extended Co islands with Co thickness as indicated by Roman numerals. For reference an $\mathrm{Au}(111)$ step edge is highlighted by the dashed red lines. (a) $I_{0}=5 \mathrm{nA} ; E_{\mathrm{WE}}=$ $-410 \mathrm{mV} ; E_{\text {tip }}=-340 \mathrm{mV}$. Bilayer Co islands nucleates at Au step edges. Plotted are the indicated line profiles 1 to 4 with respect to the marked $\mathrm{Au}(111)$ step edge in (a): (b) Monatomic Au step edge of $(2.4 \pm 0.3) \AA$ used for scanner calibration and plotted in (c-e) as well. (c) $3 \mathrm{AL} \mathrm{Co} \widehat{=}(5.4 \pm 0.3) \AA$ located at the lower Au terrace. (d) $3 \mathrm{AL}$ $\mathrm{Co} \widehat{=}(5.3 \pm 0.3) \AA$ located at the upper Au terrace. (e) From $3 \mathrm{AL}$ Co at the upper Au terrace to $3 \mathrm{AL}$ Co at the lower Au terrace via $4 \mathrm{AL}$ Co $\widehat{=}(7.4 \pm 0.3) \AA$ Despite the bare $\mathrm{Au}(111)$ area $(66.4 \%)$ the nominally deposited Co amounts to $\approx 1$ monolayer. 
Typically, deposited Co is exclusively quantified by coverage in units of monolayers. Thereby it is assumed, that deposition follows a layer-by-layer mode regularly distributed [152, 194] as it is observed for metal deposition in vacuum. Only in few cases a bilayer is initially deposited such as for Co. As mentioned above, in the experiments it is observed, that Co is deposited in extended Co islands, with a typical lateral size of $0.25 \mathrm{\mu m}^{2}$, as shown in A typical example is given in Figure 3.8(a). There the Co islands cover an area $A_{\text {Co }} \widehat{=} 0.189 \mu^{2}$ which amounts to $33.6 \%$ of the entire investigated area $A_{\text {total }} \widehat{=}(0.75 \mu \mathrm{m})^{2}$ with Co islands of $3 \mathrm{AL}$ up to $4 \mathrm{AL}$ in height $h[A L]$. The nominally deposited Co expressed in units of monolayers (ML) amounts to

$$
\frac{A_{\text {Co }} \times h[A L]}{A_{\text {total }}} \cong 1 \mathrm{ML}
$$

It is therefore not meaningful to express the Co amount in ML for locally resolved measurements on those samples. In order to avoid confusion, hereafter all data concerning the thickness of investigated Co islands are given in units of atomic layers (AL).

As described in Section 3.2.1, a vacuum-like gap is formed at a tunneling current of $I_{0,1}=10 \mathrm{nA}$, and at an adjusted bias voltage of $V_{b, 1}=100 \mathrm{mV}$, resulting in a tunneling distance $d_{1}<0.35 \mathrm{~nm}$ [93]. All subsequent sp-STM measurements were recorded at low bias voltages $V_{b, 2}$ in the range of $5 \mathrm{meV}$ to $20 \mathrm{meV}$ and tunneling current setpoints $I_{0}$ of $5 \mathrm{nA}$ to $12 \mathrm{nA}$. Since the tunneling current $I_{0}$ is proportional to the bias voltage $V_{b}$ according to Equation (3.9), the gap for subsequent sp-STM investigations at $I_{0,2}=6 \mathrm{nA}$ and $V_{2, b}=18 \mathrm{mV}$ is approximated to

$$
\frac{\ln \left(\frac{I_{0,2}}{I_{0,1}} \cdot \frac{V_{b, 1}}{V_{b, 2}}\right)}{-10.2 \cdot \sqrt{1.51 \mathrm{eV}}}+d_{1} \propto d_{2}=0.25 \mathrm{~nm} .
$$

Therefore it is most likely, that the tunneling process occurs across a vacuum barrier similarly to STM and sp-STM measurements in UHV.

\subsubsection{Morphology of $\mathrm{Co}(0001)$ on $\mathrm{Au}(111)$}

The line profiles shown the Figures 3.7 and 3.8 allow a close inspection of the morphology of the extended Co islands on $\mathrm{Au}(111)$. In both figures the line-scans indicated with (1) run across a monatomic step edge of the substrate and are used as references, $(2.4 \pm 0.3) \AA$, compare [11]. Measured with respect to the $\mathrm{Au}$ terrace directly below, the initial Co bilayer (2 AL) displays an apparent height of $(3.6 \pm 0.4) \AA$.

With respect to the underlying Co bilayer, further Co layers show as steps of $2 \AA$, corresponding to the height of a single atomic Co layer, see Figure 3.7(c) and 3.8(c-e). Thus, Co trilayers (3 AL) amount to $(5.4 \pm 0.3) \AA$, and 4 AL of Co to $(7.4 \pm 0.3) \AA$. 
These observed values agree with previous reports of electrochemically deposited Co [4].

Based on the known lattice constant in $\mathrm{Co}\langle 0001\rangle(2 \mathrm{AL} \widehat{=} 4.07 \AA)$ [7] and compared to STM measurements of small Co islands on $\mathrm{Au}(111)$ in vacuum which agree well with this value [129, 194, a bilayer of hcp structured $\mathrm{Co}(0001)$ on $\mathrm{Au}(111)$ is expected to have an apparent height of $4.1 \AA$. The corrugation resulting of the moire structure is reported in vacuum to $\sim 0.7 \AA$ on the initial Co bilayer with a periodicity $(26.0 \pm 1.5) \AA[129$ ] which fits with the moiré structure observed. The reduction of the initial bilayer height from $4.1 \AA$ to about $(3.6 \pm 0.4) \AA$ with respect to the Au surface is in the range of the corrugation of the moiré structure observed and can be therefore caused by the high mismatch of $13 \%$ between $\mathrm{Co}(0001)$ and $\mathrm{Au}(111)$ lateral lattice constants.

A further possible explanation for the reduced bilayer thickness is the formation of a $\mathrm{Co} / \mathrm{Au}$ alloy at the interface[148. Such alloying was previously observed for $\mathrm{Co} / \mathrm{Au}(111)$ electrochemically deposited [4] as well as for evaporated [121] and will therefore be closely investigated in the following.

\subsubsection{Formation of Co-Au Alloy}

Combining STM with an electrochemical cell offers real space observations during dissolution of deposited metal on an investigated working electrode surface.

The topography of a pristine $\mathrm{Au}(111)$ surface is shown in Figure 3.9(a). The investigated area is $1 \mu^{2}$ and shows the reconstructed $\mathrm{Au}(111)$ surface at $E_{\mathrm{WE}}=$ $-341 \mathrm{mV}$. After Co deposition $\left(E_{\mathrm{WE}} \approx-470 \mathrm{mV}\right)$ the substrate potential $E_{\mathrm{WE}}$ is set to $-346 \mathrm{mV}$. Subsequently, all $\mathrm{Au}(111)$ terraces are covered by extended Co bilayer islands, where the third and fourth atomic layers of Co begin to nucleate, see Figure 3.9(b). Nonetheless, the Co islands are still well separated by bare Au areas. As a guide to the eye, on one Au terrace the deposited Co is highlighted in blue.

The STM micrograph in Figure 3.9(c) is a scan from bottom to top. During the STM investigation of the surface area marked in orange, the substrate potential $E_{\mathrm{WE}}$ is varied from $-346 \mathrm{mV} \rightarrow 30 \mathrm{mV} \rightarrow-346 \mathrm{mV}$ corresponding to the simultaneous CV observation plotted in Figure $3.9(\mathrm{~d})$. The direction of this potential sweep is indicated by the orange arrow on the side of the STM image. The Co dissolution caused by the potential increase and is observed in-situ by in the STM imaging as well as in the CV by the dissolution peak A'. The average Co coverage on the total $\mathrm{Au}(111)$ surface in contact to the electrolyte $A_{\text {substrate }} \approx 0.5 \mathrm{~cm}^{2}$ can be estimated by this CV curve by integrating the measured anodic current over time, yielding the measured value of total charge $q_{\text {total }}=1.3 \mathrm{mC}$ :

$$
\frac{q_{\text {total }}}{A_{\text {substrate }}} q_{\mathrm{ML}}=4.4 \mathrm{ML} \text {. }
$$




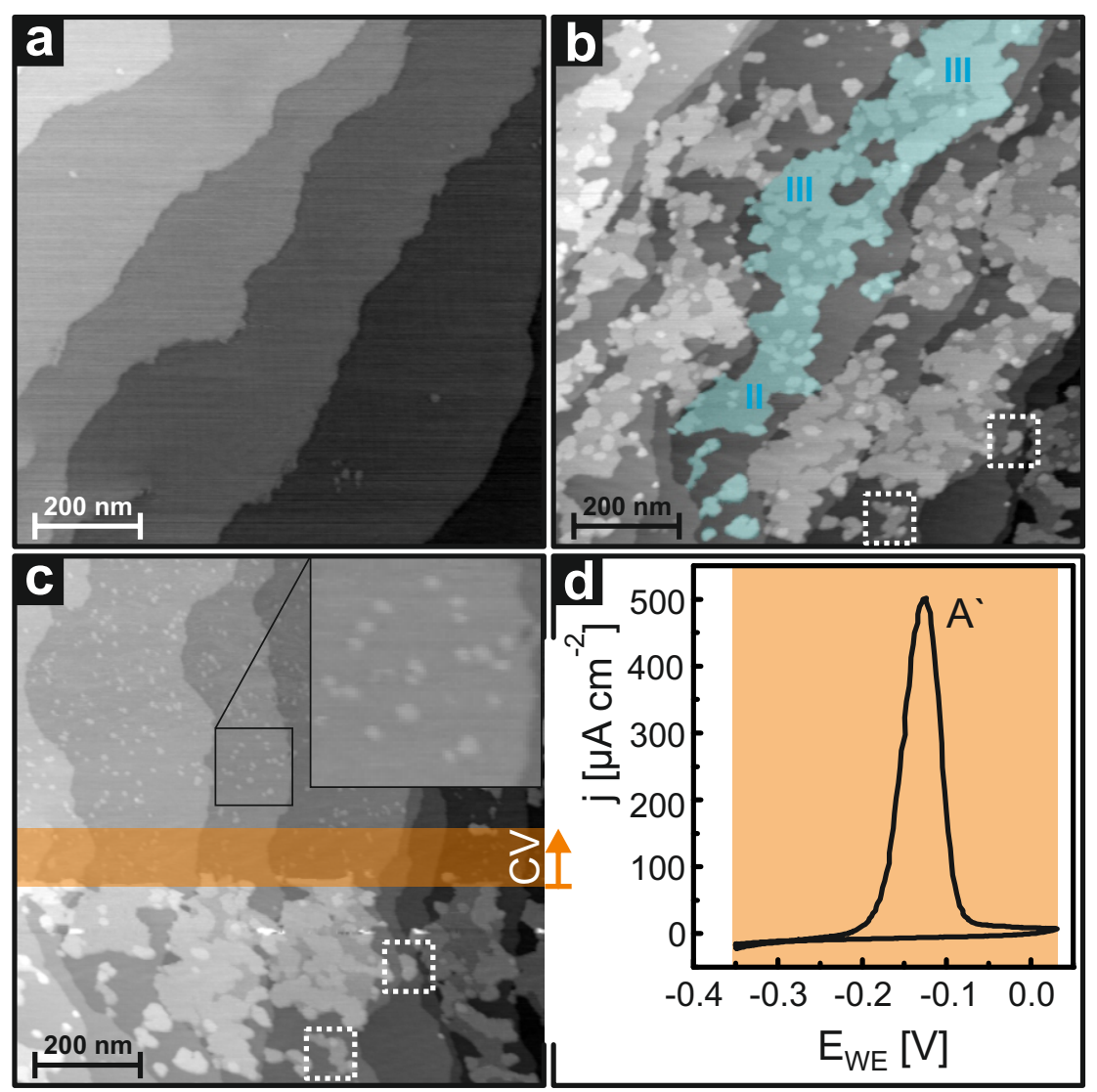

Figure 3.9: STM and CV of Co dissolution: $I_{0}=4 \mathrm{nA}, E_{\mathrm{tip}}=-369 \mathrm{mV}, E_{\mathrm{WE}}=$ $-346 \mathrm{mV}$, (a) $E_{\mathrm{WE}}=-341 \mathrm{mV}$. Bare $\mathrm{Au}(111)$ terraces before Co deposition, (b) $E_{\mathrm{WE}}=-346 \mathrm{mV}$. After Co deposition islands of $2 \mathrm{AL}$ to $4 \mathrm{AL}$ are observed, examples highlighted in blue. Dashed squares mark the same Co islands in (b) and (c). (c) During STM measurement from bottom to top the substrate potential $E_{\mathrm{WE}}$ is varied the area marked in orange corresponding to the CV plotted in (d). Whereas in the lower part of the micrograph extended Co island are observed, the $E_{\mathrm{WE}}$ increase results in dissolution of the Co islands formerly located on the upper part of the image. Co clusters are left on the $\mathrm{Au}(111)$ surface even at high potential (inset). (d) Corresponding CV measurement with $\frac{d E_{\mathrm{WE}}}{d t}=10 \mathrm{mV} \mathrm{s}^{-1}$. Co dissolution is reflected by peak A'. Thus, the average coverage of Co on the total crystal surface can be estimated to be $4.4 \mathrm{ML}$. 
Here, the charge per Co monolayer $q_{\mathrm{ML}}$ is $\left(d_{\text {surface }} \cdot n \cdot e=0.587 \mathrm{mC} \mathrm{cm}^{-2}\right)$ and the surface atomic density of $\mathrm{Co}(0001) d_{\text {surface }}=1.833 \cdot 10^{15} \mathrm{~cm}^{-2}$, calculated by the $\mathrm{Co}(0001)$ lattice constant, $2.51 \AA[7]$.

Even if most of the deposited Co is dissolved, small clusters remain on the $\mathrm{Au}(111)$ surface, as observed in the top part of the STM image in Figure 3.9.(c) and highlighted by the inset. This indicate that part of the deposited Co on $\mathrm{Au}(111)$ forms a $\mathrm{Co}-\mathrm{Au}$ alloy or results in embedded $\mathrm{Co}$ islands at the $\mathrm{Co} / \mathrm{Au}$ interface, which remain on the $\mathrm{Au}(111)$ surface even at potentials above the dissolution potential [4, 34, 121, 191. This effect becomes particularly evident after STM investigations of $\mathrm{Co}$ on $\mathrm{Au}(111)$, as Co remains up to hours on the $\mathrm{Au}(111)$ surface.

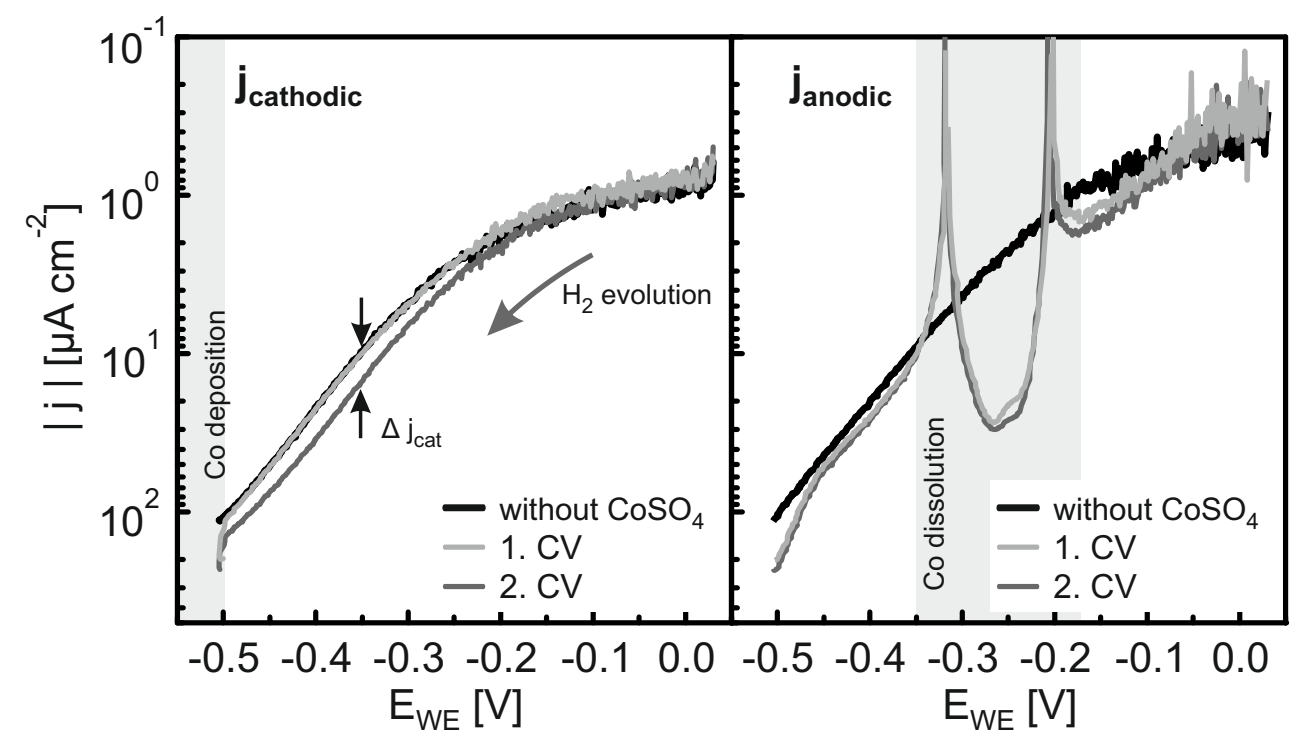

Figure 3.10: 1 . and 2. $\mathrm{CV}$ of $\mathrm{Au}(111)$ in $\mathrm{Na}_{2} \mathrm{SO}_{4}, \mathrm{H}_{2} \mathrm{SO}_{4}$ and $\mathrm{CoSO}_{4}$, separated in the cathodic $\left(j_{\text {cat }}\right.$, left) and the anodic current density $\left(j_{\text {ano }}\right.$, right). In addition, a CV of $\mathrm{Au}(111)$ without $\mathrm{CoSO}_{4}$ in the solution is shown for comparison. The Co deposition (left), and subsequent Co dissolution (right) are highlighted by gray backgrounds. In the 2.CV $j_{\text {cat }}$ is increased, even though the Co dissolution observed in $j_{\text {ano }}$ is nearly identical. This difference $\Delta j_{\text {cat }}$ is not caused by Co deposition but rather by a higher hydrogen evolution.

Cobalt and other transition metals are known to catalyze hydrogen evolution [114, 141]. Since Co remains on the substrate electrode, a higher hydrogen evolution is caused, which is also observed by $\mathrm{CV}$.

CVs of Co deposition and following Co dissolution further confirm the STM observation of Co remaining on the substrate. The first two CVs of a freshly prepared $\mathrm{Au}(111)$ surface with $\mathrm{CoSO}_{4}$ in solution are plotted in Figure 3.10, slit in cathodic current density $j_{\text {cat }}$ and anodic current density $j_{a n o}$. Even though both are measured 
in sequence, they differ in the lower potential range. The cathodic current density (decreasing potential $E_{\mathrm{WE}}$ ) of the second cycle is markedly higher in the potential range from $-220 \mathrm{mV}$ to $-500 \mathrm{mV}$, and during Co bulk deposition. However, for both CV cycles the dissolution peak A' is nearly identical, and thus, a higher Co deposition can not be the reason for the increased cathodic current density at low potentials. Instead this stems from a stronger higher hydrogen evolution at the working electrode $W E_{\text {sub }}$ during the second $\mathrm{CV}$ which can be catalyzed by Co.

As a consequence of these observations by CV and ec-STM all subsequent samples of Co islands on $\mathrm{Au}(111)$ in this work are investigated after initial Co deposition on a freshly prepared $\mathrm{Au}(111)$ surface. Hence, to prevent any unintended Co deposition during the ec-STM, the aforementioned precautions were taken. Following this preparation routine stable Co islands were electrochemically deposited on the pristine $\mathrm{Au}(111)$ surface.

\subsection{Conclusion}

Given the observations in Chapter 2, appropriate preparation parameters for electrochemical deposition of $\mathrm{Co}$ onto $\mathrm{Au}(111)$ were established. For sample preparation, the $\mathrm{Au}(111)$ substrate potential was decreased below the Nernst potential $E_{0}\left(C o / C^{2+}\right)$ to $E_{\mathrm{WE}} \approx-470 \mathrm{mV}$, while Co deposition was monitored in-situ by fast ec-STM scan speeds. Co growth is interrupted at the desired coverage by increasing the $\mathrm{Au}(111)$ substrate potential $E_{\mathrm{WE}} \approx 370 \mathrm{mV}$ near the Nernst potential. This way, extended and stable Co islands of up to $4 \mathrm{AL}$ were deposited with remaining $\mathrm{Au}(111)$ areas in between. After this step of sample preparation, $\mathrm{Co}$ on $\mathrm{Au}(111)$ can be investigated by ec-STM and further sp-STM.

In contrast to previous investigations, the observed Co deposition at the solid/liquid interface in this work occurs by lateral growth of bilayer high islands originating from $\mathrm{Au}(111)$ step edges, where additional Co layers are deposited on top before the initial Co bilayer islands coalesce forming a completed bilayer.

Co deposition on $\mathrm{Au}(111)$ in UHV features uniformly distributed small Co clusters on the reconstructed $\mathrm{Au}(111)$ surface located at the corners of the herringbone structure and differs clearly from the investigated Co deposition at the solid/liquid interface.

The high lateral resolution of the STM measurements at the solid/liquid interface was demonstrated by the resolution of the $\sqrt{3} \times 22$ reconstructed $\mathrm{Au}(111)$ surface as well as by the moiré structure on top of the deposited Co islands.

By combined CV and STM measurements the nominal Co coverage on $\mathrm{Au}(111)$ can be estimated. In addition, an alloy formation at the $\mathrm{Co}-\mathrm{Au}$ interface is observed indirectly by higher hydrogen evolution in $\mathrm{CV}$ in the second circle and in ec-STM observations by small clusters remaining on the substrate after Co disso- 
lution. Therefore, all investigations of $\mathrm{Co}$ on $\mathrm{Au}(111)$ were performed on a freshly prepared sample.

The adjustment of a low bias voltage in the range of $20 \mathrm{mV}$ as well as high tunneling currents in the range of $5 \mathrm{nA}$ ensure a vacuum-like gap between tip and substrate. This is an imported aspect especially for sp-STM at the solid/liquid interface. 
3. STM 


\section{Chapter 4}

\section{Spin-polarized Scanning Tunneling Microscopy}

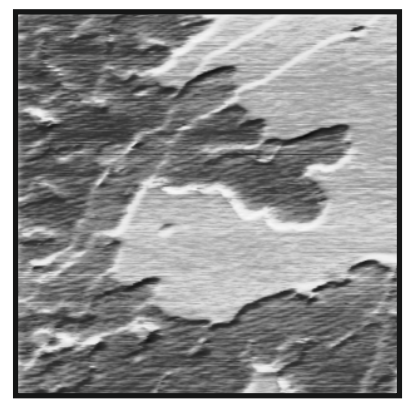

Spin-polarized scanning tunneling microscopy (sp-STM) is a powerful tool to investigate magnetic properties of magnetic surfaces [198, [46] and nanostructures [45] [151] [172] in-situ with high lateral resolution. Magnetic properties of structures prepared at the solid/liquid interface can be investigated in-situ by integral techniques like the magneto-optical Kerr effect [2, 146, 161, 162] or alternating gradient field magnetometry [5, 33, 73 .

In this chapter sp-STM at a solid/liquid interface is described and further performed in order to simultaneously investigate the structure and magnetization of electrochemically deposited magnetic films. At the beginning, a theoretical background of sp-STM is given and different modes of operation are introduced. Detailed descriptions can be found elsewhere [23, 130, 199]. The implementation of a sp-STM setup, which was constructed as part of this thesis, within the electrochemical cell is explained. In order to perform stable sp-STM measurements magnetic sp-STM tips should meet some requirements. First of all a vanishing magnetostriction effect is desired to prevent damage to the magnetic tip and to enable sp-STM. In addition, a low coercive field strength of the tip material is required. Detailed investigations on differing magnetic sp-STM tips and their magnetic properties are presented in Section 4.3. Finally, electrochemically deposited $\mathrm{Co}$ on $\mathrm{Au}(111)$ is investigated by sp-STM as a model system using the differential magnetic mode technique established by Wulfhekel and Kirschner 202 . 


\section{$4.1 \quad$ Sp-STM - Theoretical Approach}

Following the experimental work of Julliere in 1975 [100] and the theoretical prediction of Slonczewski in 1989 [175] the magnetoresistance caused by the spin-polarized conductance of a ferromagnet-insulator-ferromagnet $\left(\mathrm{FM}_{A}-\mathrm{I}_{-}-\mathrm{FM}_{B}\right)$ tunneling junction was investigated. Two ferromagnetic electrodes, $\mathrm{FM}_{A}$ and $\mathrm{FM}_{B}$, separated by an insulator I are schematically shown in Figure 4.1(a). In this model system the total tunneling conductance $G$ is given by

$$
G=G_{0} \cdot\left(1+P_{A} P_{B} \cos (\theta)\right)=\underbrace{G_{0}}_{\text {1. term }}+\underbrace{G_{0} P_{A} P_{B} \cos (\theta)}_{\text {2. term }}=G_{0}+G_{s p}
$$

The first term is a spin-averaged tunneling conductance, the second term describes the spin-polarized conductance in dependence of the relative orientation between the magnetizations $\theta$. The dimensionless spin-polarization $P_{i}$ of either of the electrodes $A$ or $B$ depends on the spin-dependent ratio of the density of states $\rho_{i}$

$$
P_{i}=\frac{\rho_{i} \uparrow-\rho_{i} \downarrow}{\rho_{i} \uparrow+\rho_{i} \downarrow}, i=A, B .
$$

According to Equation (4.1) the total conductance $G=\frac{1}{R}$ shows a cosine dependence due to the relative orientation of magnetization $\vec{M}_{A}$ to magnetization $\vec{M}_{B}$ between both ferromagnetic electrodes $\mathrm{FM}_{A}$ and $\mathrm{FM}_{B}$, as shown in Figure 4.1.
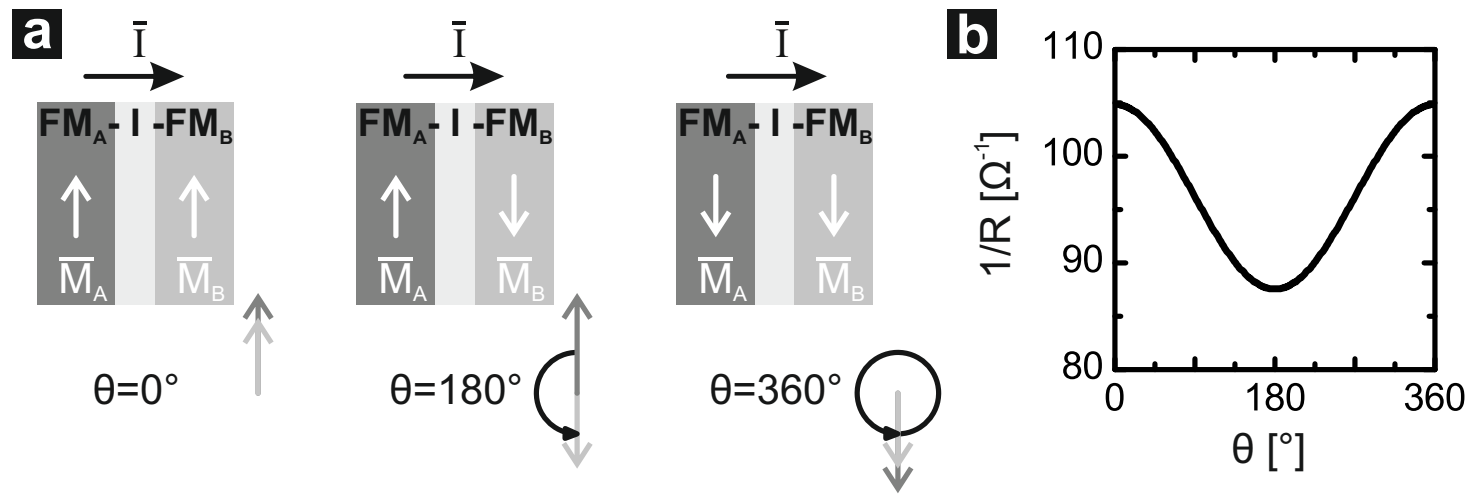

Figure 4.1: (a) Ferromagnet-insulator-ferromagnet junction with variable angle $\theta$ between magnetization $\vec{M}_{A}$ and magnetization $\vec{M}_{B}$ of the two electrodes $A$ and $B$. (b) Investigations of the tunneling conductance $G=\frac{1}{R}$ reveal a cosine dependence due to the relative orientation between the magnetizations expressed by the angle $\theta$, see Miyazaki and Tezuka [127.

The tunneling process in case of an antiparallel $\left(\theta=180^{\circ}\right)$ or a parallel $(\theta=$ $\left.0^{\circ} ; 360^{\circ}\right)$ orientation of the magnetizations is illustrated in Figure 4.2. A simplified 
a

antiparallel orientation

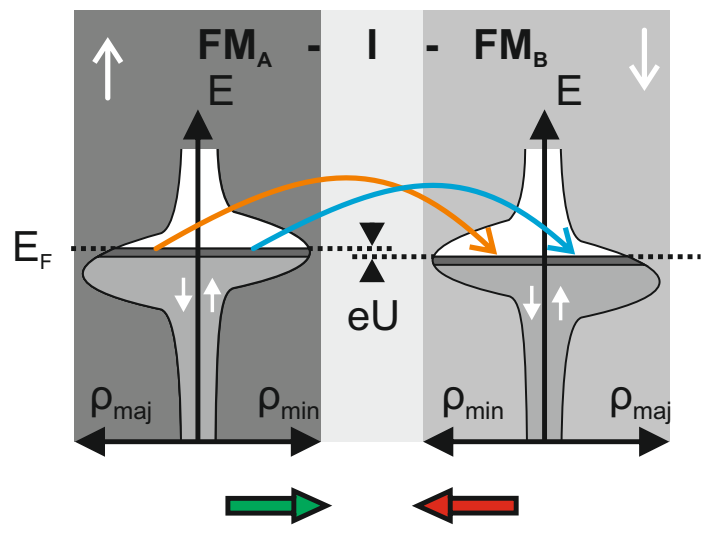

b parallel orientation

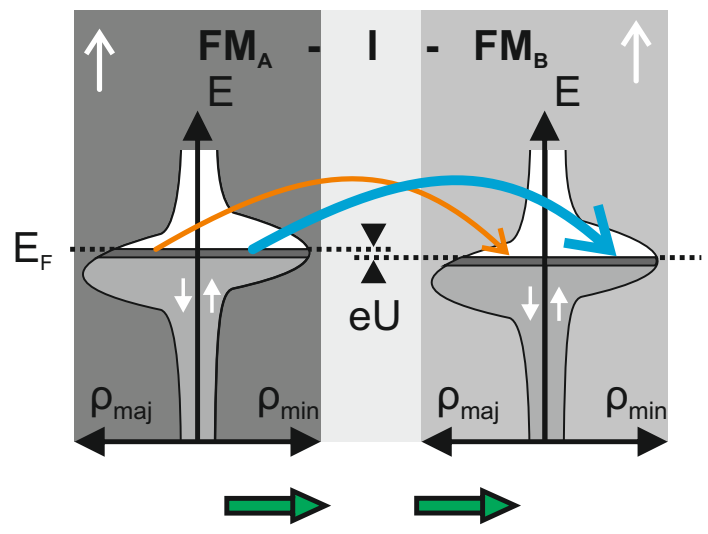

Figure 4.2: The schematic band structure for the $3 d$ shell is pictured of two ferromagnetic electrodes $\mathbf{F} \mathbf{M}_{A}$ and $\mathbf{F} \mathbf{M}_{B}$ in (a) antiparallel and (b) parallel orientation separated by an insulator $\mathbf{I}$ in between. Caused by an applied bias voltage a net tunneling current occurs. The total density of states is simplified and its splitting into majority $\downarrow$ and minority $\uparrow$ states. Assuming that electron spin is conserved during tunneling $\left(\rho_{A, \downarrow} \rightarrow \rho_{B, \downarrow}\right.$ and $\left.\rho_{A, \uparrow} \rightarrow \rho_{B, \uparrow}\right)$, in parallel orientation a higher tunneling current is measured.

band structure for the $3 d$ shell of transition metals such as $\mathrm{Ni}, \mathrm{Co}$, and Fe is shown, split into majority and minority band [185]. Both ferromagnetic electrodes $\mathrm{FM}_{A}$ and $\mathrm{FM}_{B}$ are separated by an insulator I, for example $\mathrm{Al}_{2} \mathrm{O}_{3}$ [128] or a vacuum gap [175]. A net magnetization of each electrode in direction of the minority spin results from an imbalance between majority $\left(\rho_{\text {maj }}\right)$ and minority electrons $\left(\rho_{\text {min }}\right)$, according to the Stoner model [185], leading to the expression of band-ferromagnetism. This model for spin-polarized tunneling assumes, that the electron spin is conserved during the tunneling process, and that only electrons close to the Fermi edge $\left(E_{F}\right)$ contribute to the tunneling current $I$. Therefore, a lower tunneling current $I_{a}$ results in case of an antiparallel orientation of the electrodes, compared to the tunneling current of a parallel orientation $I_{p}$, thus $I_{a}<I_{p}$.

The total tunneling current $I$ of the tunneling junction follows from Equation (4.1) for the total conductance $G$ as

$$
I=I_{0} \cdot\left(1+P_{A} P_{B} \cos (\theta)\right)=I_{0}+I_{0} P_{A} P_{B} \cos (\theta)=I_{0}+I_{s p},
$$

with the spin-average tunneling current $I_{0}$ and the spin-polarized tunneling current $I_{s p}$. Thus, in case of an anti-/parallel orientation between both electrodes the tunneling current equals

$$
I_{s p}= \begin{cases}I_{a}=I_{0} \cdot\left(1-P_{A} P_{B}\right), & \text { for } \theta=180^{\circ} \\ I_{p}=I_{0} \cdot\left(1+P_{A} P_{B}\right), & \text { for } \theta=0^{\circ}\end{cases}
$$




\section{a}

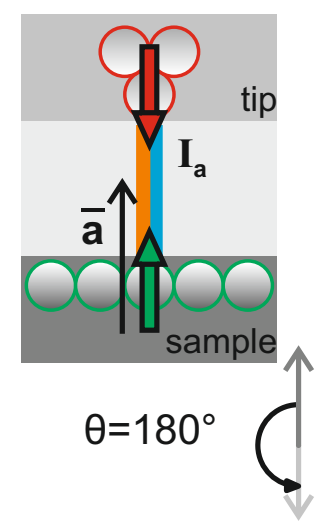

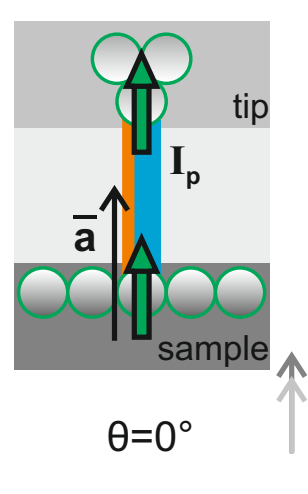

\section{b}

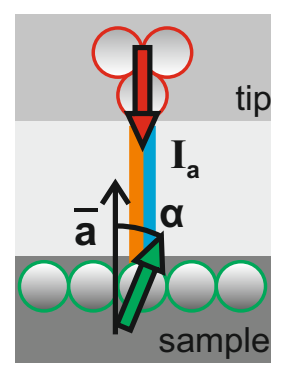

$\theta=180^{\circ}+\alpha$

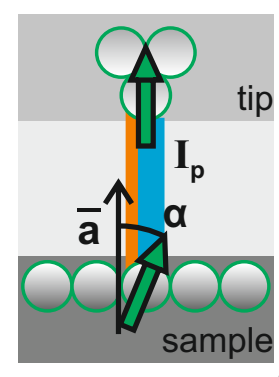

$\theta=0^{\circ}+\alpha$

Figure 4.3: Illustrated dependence of spin-polarized tunneling current on the alignment of magnetization. (a) In full antiparallel (left)/ parallel (right) orientation between tip electrode and sample electrode with $\theta=180^{\circ} / 0^{\circ}$. (b) Since sample magnetization is tilted by $\alpha$ with respect to the surface normal, the difference between spin-polarized tunneling currents $I_{a}$ and $I_{p}$ is lowered.

These orientations and the resulting tunneling current are illustrated in Figure 4.3 (a), where the magnetic STM tip and the magnetic substrate act as ferromagnetic electrodes. In this example, an out-of-plane magnetization of the sample is assumed along the surface normal $\vec{a}$. The tip magnetization is also aligned along $\vec{a}$. Thus, caused by the relative orientation of both magnetizations, the tunneling current has a maximum value for orientation, and a minimum value for antiparallel orientation. Deviations from the ideal case (anti-/parallel) leads to a decreasing difference between both extreme values (minimum/maximum), which is illustrated in Figure 4.3(b). When the magnetization of the sample is tilted by an angle of $\alpha$ with respect to the surface normal $\vec{a}$ while the tip magnetization remains aligned to $\vec{a}$, a lowered magnitude of the spin-polarized component of the tunneling current results. In a perpendicular orientation, the spin-polarized tunneling current equals zero:

$$
\begin{array}{ll}
I=I_{p}=I_{0} \cdot\left(1+P_{A} P_{B} \cos (\alpha)\right) & \text { for }-90^{\circ}<\theta<90^{\circ} \\
I=I_{a}=I_{0} \cdot\left(1-P_{A} P_{B} \cos (\alpha)\right) & \text { for } 90^{\circ}<\theta<270^{\circ} \\
I=I_{0} ; \text { with } I_{s p}=0 & \text { for } \theta= \pm 90^{\circ}
\end{array}
$$

\subsection{Sp-STM Implementation}

Currently sp-STM is accomplished by three different modes of operation for investigating magnetic surfaces using magnetic tips. A short summary is given in this section. More details are available in literature published by Bode [23], Wiesendanger, R. [199] and Hopster and Oepen [91], a more theoretical description can be found in Wortmann et al. [200]. 


\subsubsection{Modes of Operation}

An early example for sp-STM was demonstrated by Wiesendanger et al., using a magnetic STM tip and STM in the constant-current mode to investigate a $\mathrm{Cr}(001)$ surface [198]. $\mathrm{Cr}(001)$ is an anti-ferromagnet, where ferromagnetic terraces are separated by atomic steps [21]. Since the STM tip magnetization is preserved throughout the entire measurement, STM investigations correspond not only to the topography of the $\mathrm{Cr}(001)$ surface, but also a spin-polarized component of the tunneling current contributes. However, it is difficult to distinguish topographical from spin-polarized information in this mode of operation. In order to extract the spin-polarized component of the tunneling current, the difference between measurements with a magnetic and a non-magnetic tip of the same surface area has to be determined. This is experimentally challenging due to the volatility of the STM tip.

A separation between topographical and magnetic information during the same measurement is an advantage of sp-STM in the spin-resolved spectroscopic mode as well as in the differential magnetic mode.

The spin-resolved spectroscopic mode of sp-STM is realized by applying an ACcomponent to the bias voltage $V_{b}$ [199]. Since the modulation frequency of the AC-bias voltage is significantly higher than the frequency of the feedback loop of the STM setup, the observed time averaged tunneling current is kept constant at the adjusted set-point. By lock-in technique at the modulation frequency, a modulated component of tunneling current is investigated, which is proportional to the energy resolved local density of states of a magnetic sample. Disadvantages of sp-STM in spin-resolved spectroscopic mode are longer measurement times and a high volume of data. In addition, a very stable STM set-up is required to prevent a drift between STM tip and investigated sample.

Sp-STM in differential magnetic mode was first described by Johnson and Clarke [98] and established by Wulfhekel and Kirschner [202]. Thereby, the tip magnetization $\vec{M}_{\text {tip }}$ is manipulated by an external magnetic field of a coil that is located around the tip. Via a modulated magnetic field the direction of the tip magnetization $\vec{M}_{\text {tip }}$ is periodically switched between parallel or antiparallel orientation $\left(\theta=0^{\circ}\right.$ or $180^{\circ}$ ) with respect to the surface normal $\vec{a}$. Using a magnetic tip, as illustrated in Figure 4.3, the sp-STM setup is sensitive to sample magnetization in out-of-plane direction $\vec{M}_{\text {sub }}$, where $\alpha \neq 90^{\circ}$ with respect to the surface normal $\vec{a}$. In-plane magnetized sample surfaces with $\alpha=90^{\circ}$ can only be investigated with STM tips where the tip magnetization $\vec{M}_{\text {tip }}$ is aligned perpendicular to the surface normal $\vec{a}$ [168]. Since the tunneling current $I$ depends locally on the orientation of tip magnetization $\vec{M}_{\text {tip }}$ relative to the investigated surface magnetization $\vec{M}_{\text {sub }}$, modulating the coil magnetic field causes a periodically manipulated tip magnetization $\vec{M}_{\text {tip }}^{f_{\text {mod }}}$, and therefore the spin-polarized component of the tunneling current $I_{s p}$ is modulated along the same lines. This modulation is observable by the lock-in technique at the 
modulation frequency $f_{\text {mod }}$, where the magnitude is given by the difference of the tunneling current $\triangle I_{s p}^{f_{\text {mod }}}$ between the antiparallel and parallel orientation

$$
\triangle I_{s p}^{f_{\text {mod }}}=I_{p}-I_{a}=2 I_{0} P_{\text {tip }} P_{\text {sample }} \cos (\alpha),
$$

which is proportional to the spin-polarization of the system tip/sample.

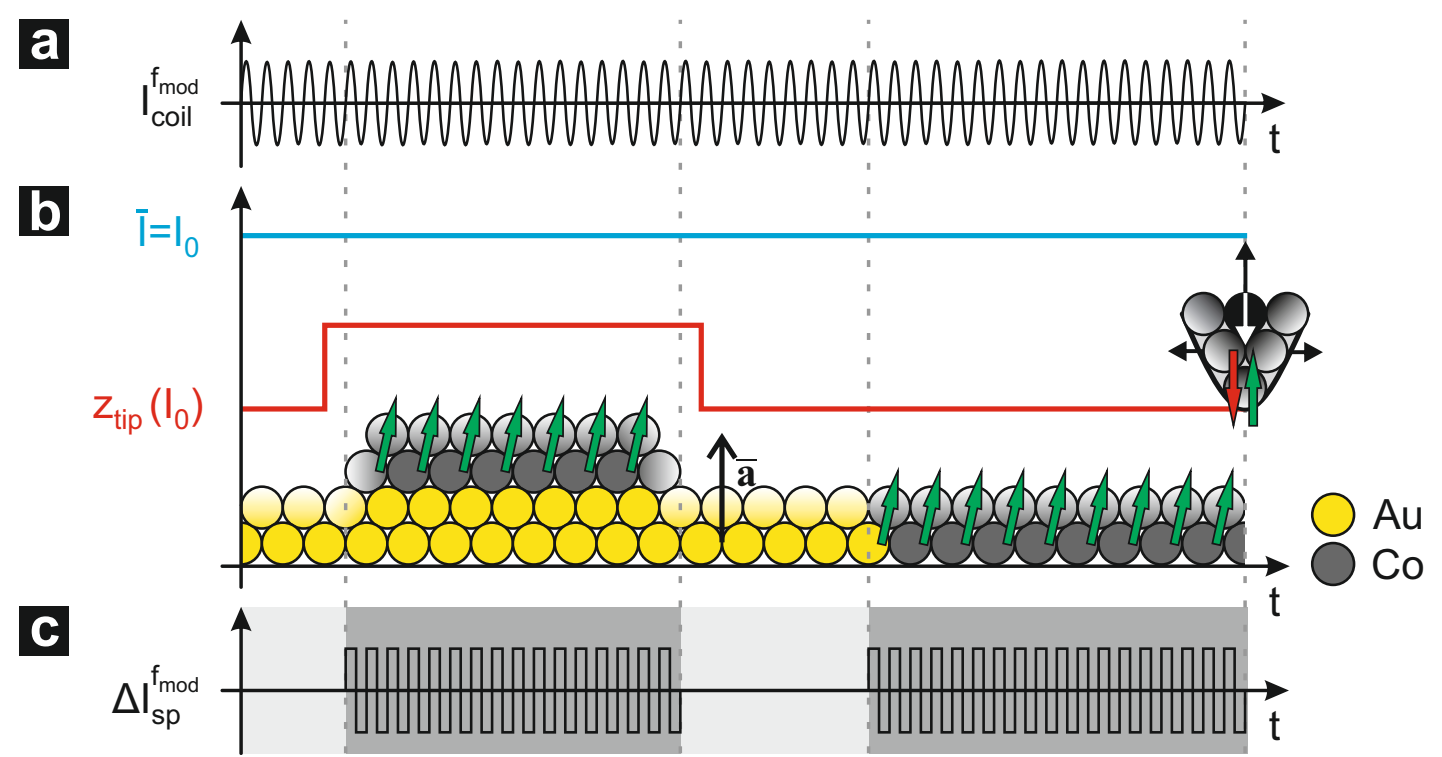

Figure 4.4: Illustrated sp-STM in differential magnetic mode: (a) Tip magnetization $\vec{M}_{\text {tip }}^{f_{\text {mod }}}$ is switched periodically by the magnetic field of a current modulated coil at the modulation frequency $f_{\text {mod }}$. (b) Time averaged tunneling current is equal to the setpoint in constant-current mode: $\bar{I}=I_{0}$. (c) The alternating magnetoresistance causes a modulated spin-polarized component of the tunneling current at the modulation frequency $f_{\text {mod }}$. The detection of $\Delta I_{s p}^{f_{\text {mod }}}$ is achieved by lock-in technique.

An illustration of sp-STM in differential magnetic mode is shown in Figure 4.4. Operating the coil with a sinusoidal current at the modulation frequency $I_{\text {coil }}^{f \text { mod }}$, plotted in Figure 4.4 (a), results in an alternating magnetic field at the modulation frequency $f_{\text {mod }}$. The magnetic field strength manipulates the tip magnetization, thereby its direction is periodically switched at $f_{\text {mod }}$ between anti-/parallel orientation with respect to the surface normal $\vec{a}$ throughout the entire STM investigation, which is illustrated in Figure 4.4(b). Since the applied modulation frequency $f_{\text {mod }}$ of the coil, and therefore of the spin-polarized tunneling current, is higher than the frequency of the STM feedback loop $f_{\mathrm{STM}}$, the STM setup detects a time averaged tunneling current $\bar{I}$

$$
\bar{I}=I_{0},
$$

which is constant and equal to the selected tunneling current set-point. Sp-STM in differential magnetic mode can also be applied to investigate sample topography 
without including further information about spin-polarization, since topographical information are observable by the time averaged $\bar{I}$. According to the switching between antiparallel and parallel orientation with $f_{\text {mod }}$, the spin-polarized component of tunneling current $I_{s p}^{f_{\text {mod }}}$ is also modulated at this frequency. Since only $\Delta I_{s p}^{f_{\text {mod }}}$ depends on the surface magnetization $\vec{M}_{\text {sub }}$, the spin-polarized component can be deconvoluted via lock-in technique at the modulation frequency $f_{\text {mod }}, 4.4$ (c), where the phase adjustment of the lock-in is set to maximize the output.

The tunneling current of the sp-STM in the differential magnetic mode can be described by two components

$$
I_{\text {total }}=I_{0}+I_{s p}^{f_{\text {mod }}} .
$$

The fist term is the spin-averaged, and thus constant, component $I_{0}$. The secondary one is the spin-polarized component $I_{s p}^{f_{\text {mod }}}$, which is related to the modulation frequency $f_{\text {mod }}$. In case of a sinusoidally modulated magnetic field, the resulting tip magnetization depends on the magnetic properties of the tip material in use. This topic is detailed further in Section 4.3.1.

\subsubsection{Sp-STM Integration}

In the present STM setup within the electrochemical cell, sp-STM in the differential magnetic mode is implemented. Therefore, a selfmade coil ${ }^{1}$ is permanently installed centered around the end of the tip, as shown in Figure 4.5. The coil body is made of aluminum, thereby it can be also used for electrical grounding. A thin insulated copper cable ${ }^{2}$ is wound around the center axis of the coil body. The upper part of the coil (close to the scanner unit) and all four sides are magnetically shielded by permalloy ${ }^{3}$. In this way the influence of the magnetic field of the coil on the STM setup is reduced. Additionally, stray magnetic field from the coil, and thus an influence on the investigated substrate is minimized. A stainless steel cover (electrically grounded) protects the coil from moisture and electrolyte splashes. A direct contact between the aluminum coil body and the inserted STM tip is avoided by a MACOR shield 4 . Because of the permanently installed coil, only the STM tips have to be prepared prior each measurement.

A schematic of the sp-STM setup implemented into the electrochemical environment is shown in Figure 4.6. The coil, installed permanently and connected at the scanner unit, is operated with a frequency generator (FG) at a modulation frequency higher than the frequency of the STM feedback loop $f_{\text {mod }}>>f_{\mathrm{STM}}$. A direct contact between coil and electrolyte solution has to be avoided, in order to prevent a

\footnotetext{
${ }^{1}$ with length $l=3.5 \mathrm{~mm}$ and middle radius $\bar{r}=\frac{r_{o}-r_{i}}{2}+r_{i}=2.65 \mathrm{~mm}$, see Figure 4.5(b)

${ }^{2} 250 \mu \mathrm{m}$ in diameter, number of turns is $N=101$.

${ }^{3}$ Permalloy is a magnetic alloy of $\mathrm{Ni}$ and $\mathrm{Fe}$, which has a high magnetic permeability.

${ }^{4} \mathrm{MACOR}$ is a kind of ceramic. Tube length $l=3 \mathrm{~mm}$
} 
Figure 4.5: (a) Aluminum coil body. The coil itself is made of copper wire wounded around the center axis at (1). Upper part and all four sides of the coil (2) are magnetically shielded by permalloy. The tip holder of the STM setup (3) is introduced from the top of the coil, where the coil body is connected to the scanner unit. A stainless steel cover (4) protects the coil from the electrochemical environment. A magnetic STM tip passed through a MACOR shield is inserted through the center at the lower end (5) of the coil into the tip holder. (b) Sketch of the sp-STM setup with integrated tip.

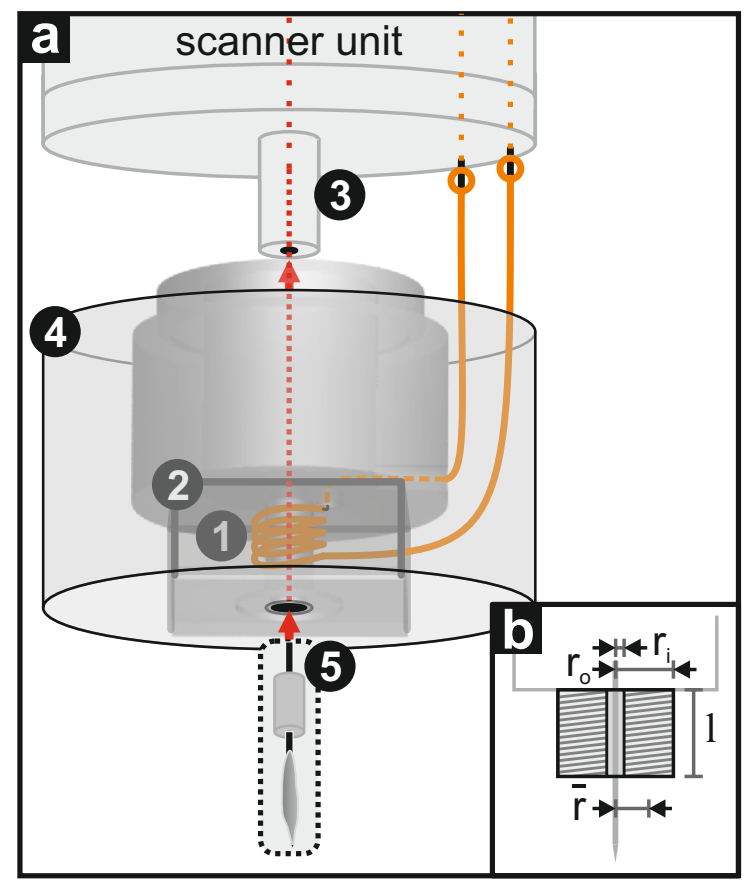

electric contact arising between STM tip and coil body. A spin-polarized component of the tunneling current occurs located above a sample surface out-of plane magnetized caused by the manipulated STM tip magnetization. The detection of this component is accomplished by lock-in technique at the modulation frequency $f_{\text {mod }}$ by synchronizing the oscillator of the lock-in to the frequency generator. In addition to the typical STM information $\bar{I}(x, y)$, the lock-in information $\Delta I_{s p}^{f_{\text {mode }}}(x, y)$ is transmitted to the STM controlling unit (STM CU) using an auxiliary input channel. Further, the measured values $\bar{I}(x, y), z(x, y)$, and $\Delta I_{s p}^{f_{\bmod }}(x, y)$ are transmitted to a $\mathrm{PC}$.

During operation the resistance of the coil increases due to resistive heating. To avoid a decreasing magnetic field over time the coil is driven by a current source

$$
I_{\text {coil }}^{f_{\text {mod }}}=I_{0, \text { coil }} \cdot \sin \left(2 \pi f_{\text {mod }} t\right) \text {. }
$$

The magnetic field strength along the $z$-direction (center axis) generated by the coil can be estimated as

$$
H(z)=I_{0, \operatorname{coil}} \frac{N}{2 l}\left(\frac{\frac{l}{2}+z}{\sqrt{\bar{r}^{2}+\left(\frac{l}{2}+z\right)^{2}}}+\frac{\frac{l}{2}-z}{\sqrt{\bar{r}^{2}+\left(\frac{l}{2}-z\right)^{2}}}\right) .
$$

However, caused by the additional permalloy shields the actual magnetic field strength of the coil is influenced. Same measurements for estimation using a Hall probe are shown in Figure 4.13(a) of the following section. 


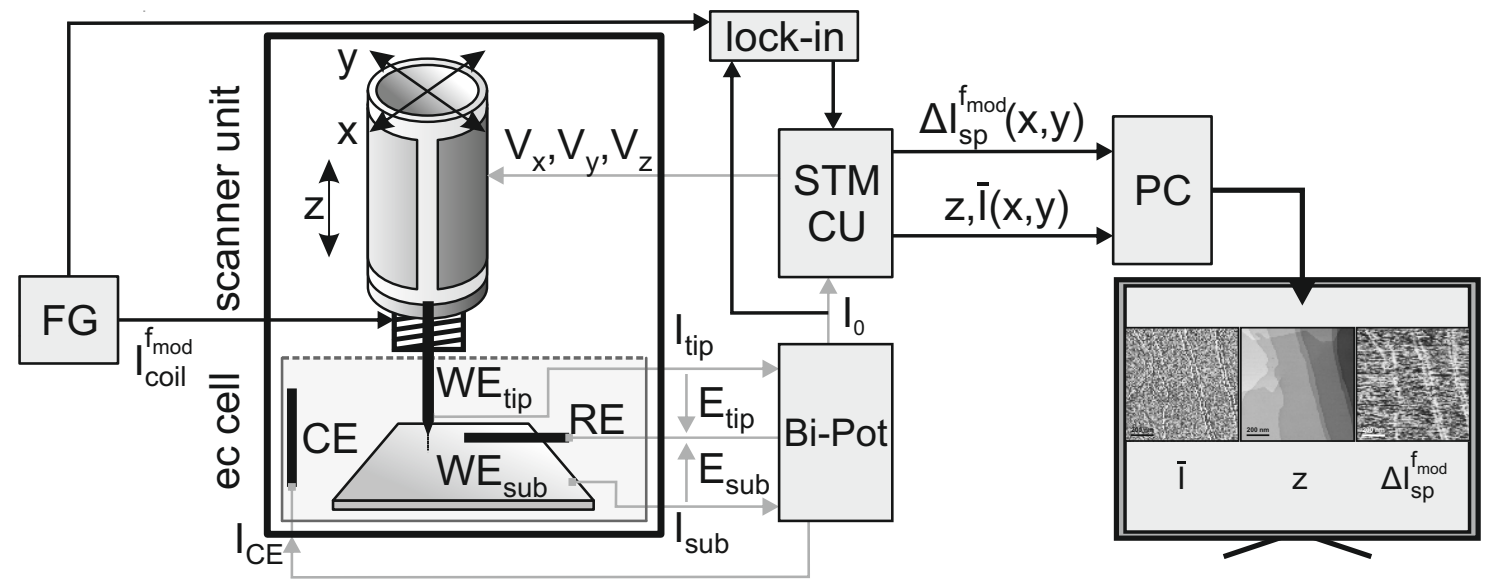

Figure 4.6: The already introduced STM setup within the electrochemical cell becomes upgraded to a sp-STM setup by integration of a coil. The operation frequency $f_{\text {mod }}$ is generated by a frequency generator (FG), which is also used to synchronized the lock-in oscillator. Thus, signals of tunneling current at $f_{\text {mod }}$ can be detected and are recorded in parallel to the STM investigations.

\subsection{Magnetic Tips}

In order to perform stable sp-STM measurements a magnetic sp-STM tip should meet certain requirements. A very important point is a negligible magnetostriction effect of the sp-STM tip in use to prevent damage to the tip arising from tip crashes during magnetization modulation $\vec{M}_{\text {tip }}^{f_{\text {mod }}}$ by the external field. Magnetostriction is an effect of second order [144], therefore it occurs at $2 f_{\text {mod }}$. Furthermore, a low coercive field strength of the tip material is required due to the required switching of tip magnetization $\vec{M}_{\text {tip }}$ at sufficient low magnetic field strength.

Some theoretical principles of the magnetostriction effect are detailed in Section 4.3.1. Two kinds of STM setups to observe tip magnetostriction are detailed in Section 4.3.2. Various tip materials of interest are investigated initially by SQUID ${ }^{1}$ and by STM-magnetostriction measurements in Section 4.3.3. Due to the required potential in the range of $-700 \mathrm{mV}$ vs SCE sp-STM tips made of $\mathrm{Ni}$ and Co-rich $\mathrm{Co}_{68,2} \mathrm{Fe}_{4,3} \mathrm{Si}_{12,5} B_{15}$ (AC20) ${ }^{2}$ are suitable choices for sp-STM at the solid/liquid interface. One disadvantage of $\mathrm{AC} 20$ is that the actual composition at the tip apex after etching is not guaranteed to be ferromagnetic since AC20 is an alloy.

\footnotetext{
${ }^{1}$ Superconducting QUantum Interference device

${ }^{2} \mathrm{Co}_{68.2} \mathrm{Fe}_{4.3} \mathrm{Si}_{12.5} \mathrm{~B}_{15}$ is a soft ferromagnetic and amorphous alloy produced by UNITIKA using an 'In-Rotation-Water-Spinning Process' [137.
} 


\subsubsection{Magnetostriction}

Different kinds of magnetostriction effects are known, here the Joule effect is discussed. The magnetostriction effect is a material property. First and foremost ferromagnetic metals [6], rare-earth metals [144], alloys of these and material compositions where any of the aforementioned are present feature magnetostriction. The magnetostriction coefficient $\lambda$ is dimensionless and defined as

$$
\lambda=\frac{\Delta L}{L} .
$$

The order of magnitude of the magnetostriction coefficient $\lambda$ varies in a wide range from $10^{-9}$ to $10^{-4}$. To get an idea of the dimension: in case of a sample rod of $10 \mathrm{~cm}$ this would correspond to length changes from $1 \AA$ to $10 \mu \mathrm{m}$. Tailor-made alloys of magnetic materials are optimized to show only vanishing magnetostriction [56, 103, 138].

Magnetostriction originates from unpaired electrons, such as the $3 d$-electrons of Fe $\left([A r] \mathbf{3 d}^{\mathbf{6}} 4 s^{2}\right)$, Co $\left([A r] \mathbf{3 d}^{\mathbf{7}} 4 s^{2}\right)$ and $\mathrm{Ni}\left([A r] \mathbf{3 d}^{\mathbf{8}} 4 s^{2}\right)$, contributing to magnetic properties [112]. Within the metal crystal the electron spin and the movement of the electrons cause magnetic fields. Because of the interaction of these magnetic fields spins of different unpaired electrons close to each other align along the same direction, with the orbitals behaving similarly if possible [52]. Groups of atoms with the same alignment are arranged in domains of one magnetization direction. In the presence of a strong external magnetic field the alignment occurs parallel to the direction of this field for anti-/ferromagnetic and ferrimagnetic materials. As a result the crystal structure extends laterally and reduces other dimensions in case of positive magnetostriction. Negative magnetostriction is the identical but inverted effect. Thus, instead of extension a contraction occurs. In both cases the magnetostrictive volume is nearly preserved [52]. For a certain magnitude of the external magnetic field, magnetostriction saturates to a corresponding value $\lambda_{\text {sat }}$. Due to the alignments of spins and orbitals within a certain crystallographic structure magnetostriction is an anisotropic effect and varies with crystallographic directions.

\begin{tabular}{ccc}
\hline$\lambda_{i i i}$ & $\mathbf{F e}$ & $\mathbf{N i}$ \\
\hline$\lambda_{100}$ & $20.7 \cdot 10^{-6}$ & $-45.9 \cdot 10^{-6}$ \\
$\lambda_{111}$ & $-21.2 \cdot 10^{-6}$ & $-24.3 \cdot 10^{-6}$ \\
\hline
\end{tabular}

Table 4.1: Saturation magnetostrictions coefficient $\lambda_{\text {sat }}$ of Ni and Fe single crystal are given for two crystallographic directions (iii), from [112].

The magnitude of the saturated magnetostriction is summarized in Table 4.1 for two crystallographic directions of Fe and Ni single crystals. A polycrystalline sample 
shows in all directions a net magnetostriction up to a saturation magnetostriction depending on the external magnetic field strength. Table 4.2 presents the saturation magnetostriction coefficient for polycrystalline materials.

\begin{tabular}{ccr}
\hline material & $\lambda_{\text {sat }}$ & reference \\
\hline $\mathrm{Co}$ & $-62 \cdot 10^{-6}$ & {$[113$} \\
$\mathrm{Co}_{68.2} \mathrm{Fe}_{4.3} \mathrm{Si}_{12.5} \mathrm{~B}_{15}(\mathrm{AC} 20)$ & $-4 \cdot 10^{-8}$ & {$[49]$} \\
$\mathrm{Fe}$ & $-7 \cdot 10^{-6}$ & {$[113$} \\
$\mathrm{Fe}_{3} \mathrm{O}_{4}$ & $40 \cdot 10^{-6}$ & {$[113$} \\
$\mathrm{Ni}$ & $-33 \cdot 10^{-6}$ & {$[113]$} \\
\hline
\end{tabular}

Table 4.2: Saturation magnetostriction coefficients $\lambda_{\text {sat }}$ for several polycrystalline materials.

a

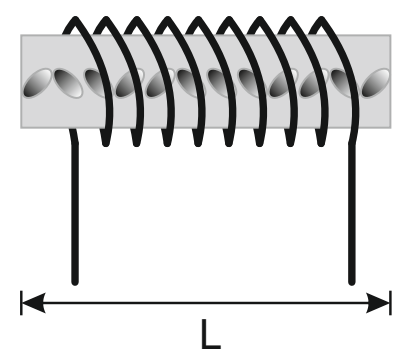

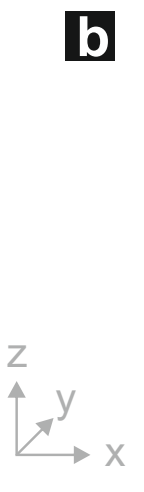

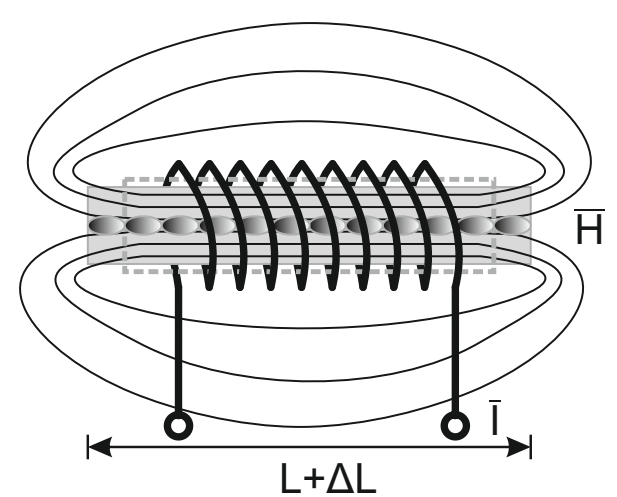

Figure 4.7: Illustration of a positive magnetostriction effect: A ferromagnetic rod is placed inside a coil (a) not in operation and (b) in operation resulting in a magnetic field. Groups of atoms with the same alignment are arranged in domains of magnetization, sketched by ovals. Once a magnetic field is applied domains are arranged along the external field, and thus an extension of $\Delta L$ is caused with concurrent reduction in diameter. The effect depends on the magnitude of the field strength along the x-axis and the magnetic properties of the magnetic rod under investigation.

The effect of magnetostriction is illustrated in Figure 4.7 by a ferromagnetic rod with positive magnetostriction placed inside a coil. Without an external magnetic field (the coil is not in operation) the ferromagnetic rod has a certain length $L$, Figure 4.7(a). During operation of the coil the rod is elongated to $L+\Delta L$ due to its magnetic properties and with $\Delta L$ depending on the magnitude of the magnetic field strength $\bar{H}$. If the direction of the magnetic field strength is reversed from $+H_{x}$ to $-H_{x}$ the magnetostriction does not change. 
The magnetostriction effect is an effect of second order, since it only depends on the magnitude of the field strength along a certain axis up to the saturation magnetostriction and not on the direction $( \pm x)[144][$ ].

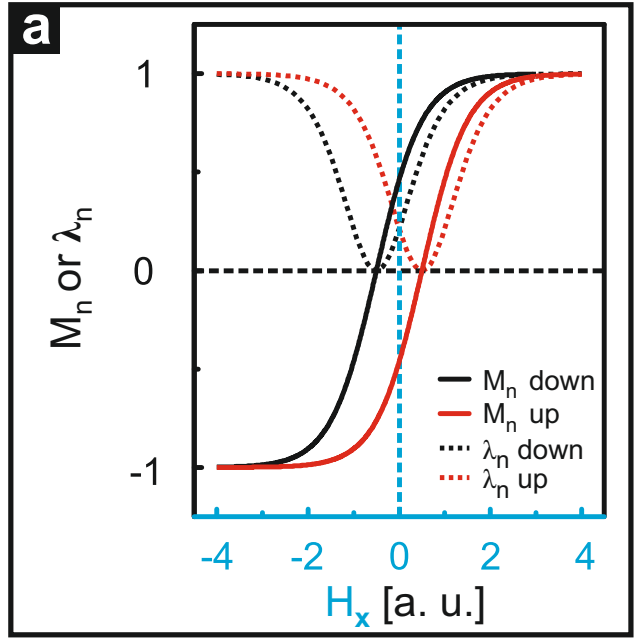

Figure 4.8: (a) Illustrated saturation hysteresis loop (solid lines) $M_{n}\left(H_{x}\right)=\frac{M_{x}\left(H_{x}\right)}{M_{x, s a t}}$ of a ferromagnetic rod and its corresponding positive magnetostriction $\lambda_{n}\left(H_{x}\right)=$ $\frac{\lambda\left(H_{x}\right)}{\lambda_{s a t}}$ (dashed lines). The saturation magnetostriction $\lambda_{\text {sat }}$ occurs at both reversal points due to the magnitude of $H_{x}$. A minimum of $\lambda$ occurs at the coercivity field strength, where $M_{x}=0$ and magnetostriction vanishes. (b) When $H_{x}$ is sinusoidally modulated (c) the magnetization $M_{x}$ continuously passes a hysteresis loop too, and (d) a modulated magnetostriction occurs with doubled frequency and with maxi$\operatorname{mum} \lambda_{\text {sat }}: f_{\lambda}^{\text {sat }}=2 f_{\mathrm{mod}}$.

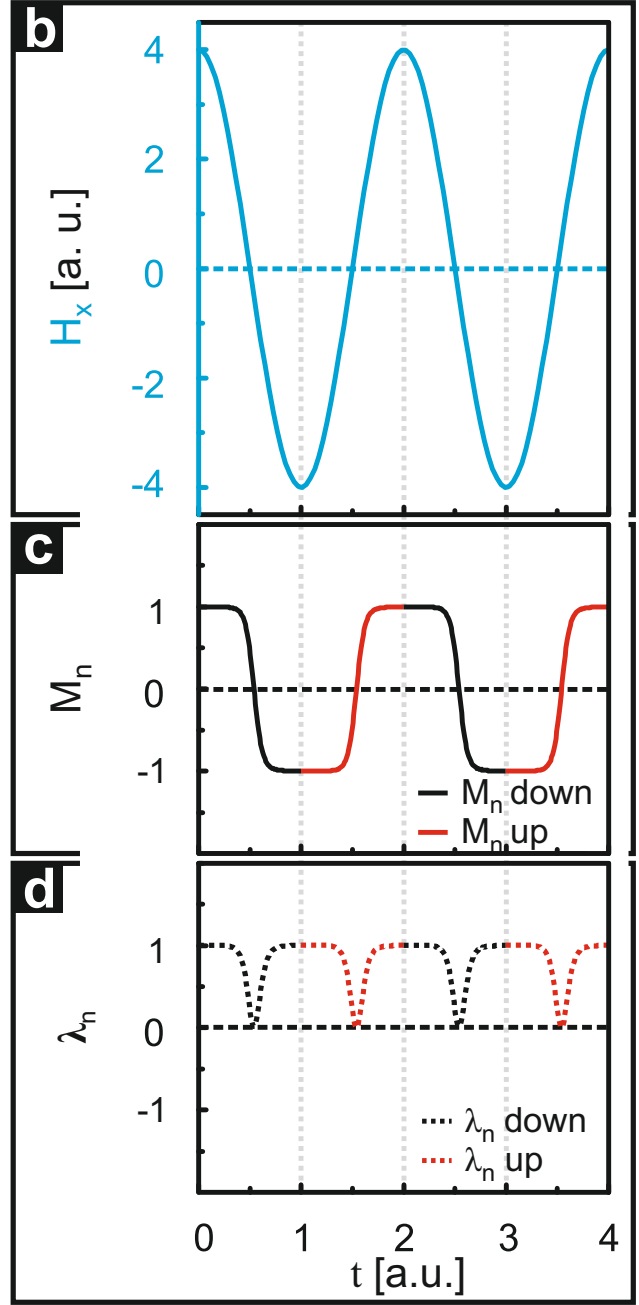

Sweeping a magnetic field strength of magnitude $\left|H_{x}\right|$ (which is high enough to switch the easy axis of magnetization $\vec{M}$ of a rod from one direction $\left(+M_{x}\right)$ to the opposite direction $\left(-M_{x}\right)$ and reverse) up and down $\left(+H_{x} \leftrightarrow-H_{x}\right)$ results in a completed hysteresis loop as illustrated in Figure 4.8(a). The saturation magnetization $M_{x, s a t}$ is reached at both reversal points $\left( \pm\left|H_{x}\right|\right)$ of the saturated hysteresis loop. The minimum of the magnetostriction is reached at the coercive field strength, $M_{x}=0$. Therefore, a sinusoidal modulation of the magnetic field strength $H_{x}(t)$, as illustrated in Figure 4.8(b), leads to a modulated magnetization $M_{x}(t)$ of the 
magnetic rod, Figure 4.8(c), and results in magnetostriction, as illustrated in Figure $4.8(\mathrm{~d})$. Since the magnetostriction depends on the magnitude of the magnetic field strength, the frequency of the magnetostriction $f_{\lambda}^{s a t}$ becomes twice the modulation frequency $f_{\text {mod }}$ when the saturation magnetostriction is reached

$$
f_{\lambda}^{s a t}=2 \cdot f_{\text {mod }}
$$

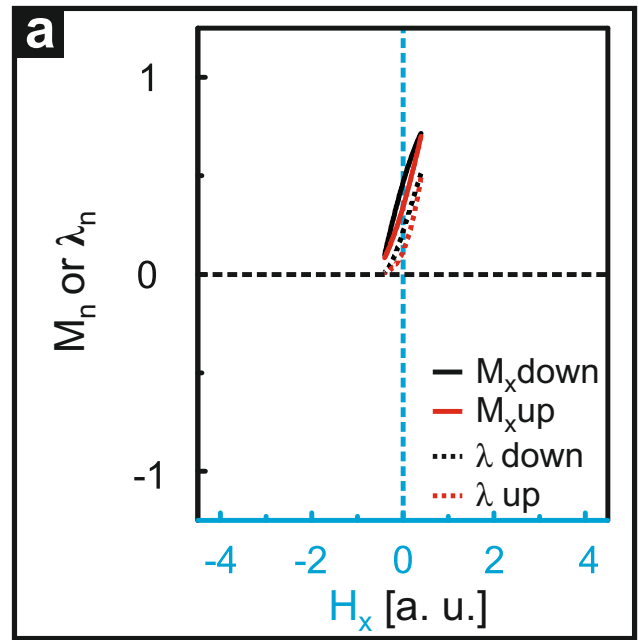

Figure 4.9: (a) Illustrated partial hysteresis loop (solid lines) $M_{n}\left(H_{x}\right)=\frac{M_{x}\left(H_{x}\right)}{M_{x, s a t}}$ of a ferromagnetic rod and its corresponding magnetostriction $\lambda_{n}\left(H_{x}\right)=\frac{\lambda\left(H_{x}\right)}{\lambda_{s a t}}$ (dashed lines). A lower magnitude of $\stackrel{\lambda}{H}_{x}$ results in a non-saturated magnetostriction $\lambda_{\text {part }}$ at both reversal points without crossing the coercive field strength, and thus, without a minimum in between. (b) Therefore, a sinusoidally modulated magnetizing force $H_{x}$ results in(c) a magnetization $M_{x}$ passing only a part of the hysteresis loop. (d) In this case a modulated magnetostriction occurs at the same frequency $f_{\lambda}^{\text {part }}=f_{\text {mod }}$.

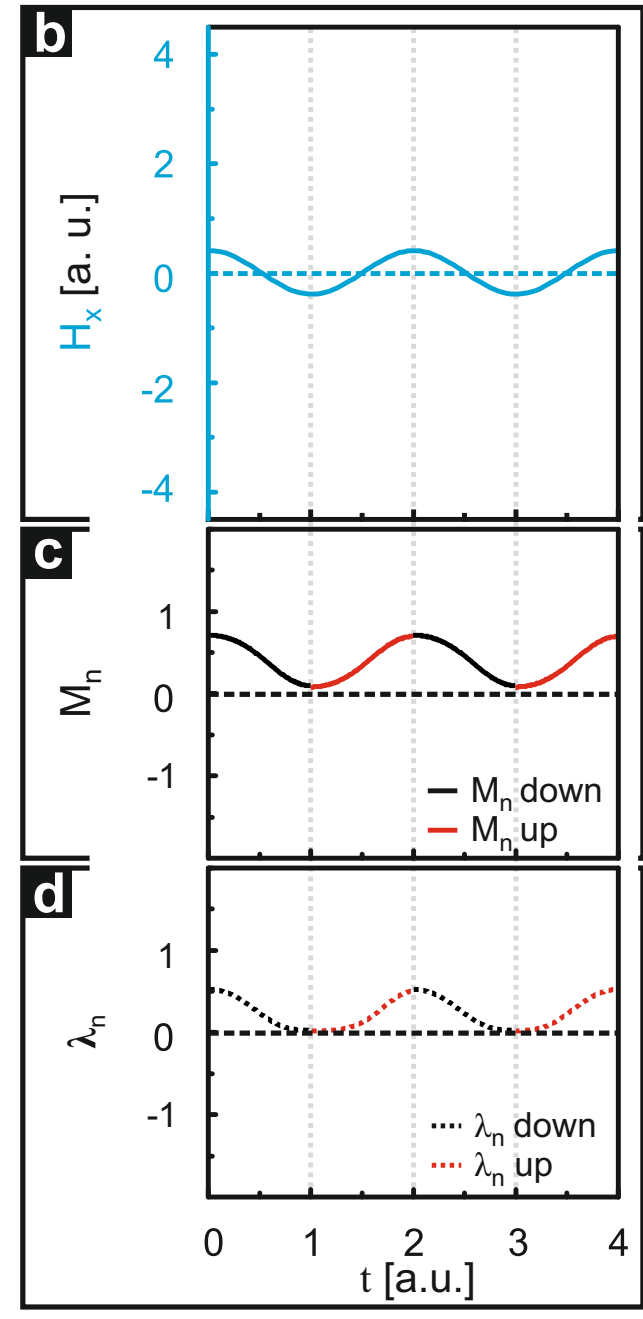

A hysteresis loop where the saturation magnetization $M_{x, s a t}$ is not reached leads to a non-saturated magnetostriction effect, exemplarily illustrated in Figure 4.9(a). The applied magnetic field strength $H_{x}(t)$ does not overcome the coercive field strength and causes a magnetization $0<M_{x}\left(H_{x}\right)<M_{x, s a t}$ which passes only through a part of the hysteresis loop. Therefore, a non-saturated magnetostriction effect is observed according to the partial hysteresis loop. The resulting frequency 
of the modulated magnetostriction $f_{\lambda}^{\text {part }}$, sketched in figure $4.9(\mathrm{~d})$, is the same as the modulation frequency of the field strength induced by the coil $f_{\text {mod }}$

$$
f_{\lambda}^{\text {part }}=f_{\text {mod }}
$$

Actually, the modulated magnetostriction of a magnetic STM tip inside a coil depends on its magnetic properties and on the magnitude of the magnetic field strength $\vec{H}$. Thereby, the modulated magnetostriction can occur with $1 f_{\bmod }$ and $2 f_{\text {mod }}$. The transition from $1 f_{\text {mod }}$ to $2 f_{\text {mod }}$ arises by intermediate states where the total modulation of the magnetostriction is a superposition of modulations with $f_{\text {mod }}$ and $2 f_{\text {mod. }}$. However, the actual magnitude of magnetostriction depends on each prepared tip.

\subsubsection{STM Setups to Observe Magnetostriction}

Due to the fact that magnetostriction is an effect of small magnitude a sensitive setup is necessary for detection. The exponential dependence of then tunneling current on the tip-sample separation $d$ in STM ensures the identification of magnetostriction even in the range of some Angstroms. Via a slight modification of the sp-STM setup, the magnetostriction of the magnetic tip can be investigated directly. Some similar concepts to investigate magnetostriction and to specify the magnetostriction coefficient were explored previously [26, 42, 52]. In this case however, the STM tip itself is the magnetostrictive and investigated sample.

Figure 4.10: Sketch of two STM setups $\boldsymbol{a}$ and $\boldsymbol{b}$ with (1) the piezoelectric element, (2) the coil for the required magnetic field, (3) the tip made of the investigated material, and (4) a nonmagnetic sample. (a) In setup $\boldsymbol{a}$ the coil is positioned around upper end of the tip. (b) In setup $\boldsymbol{b}$ the coil is positioned around the sample, and thus

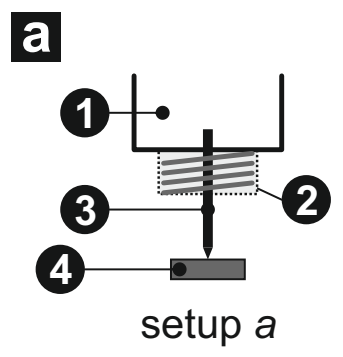

b

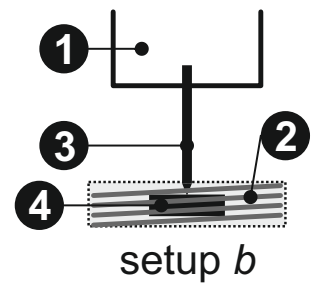
around the tip apex during tunneling contact.

Two sp-STM setups allow probing of the magnetostriction effects of various tip materials, see Figure 4.10. Setup $\boldsymbol{a}$, where a coil is positioned around the upper end of the tip, is used for tips made of one magnetic metal/alloy over the entire length such as pure $\mathrm{Ni}$ or AC20 tips. In contrast, in setup $\boldsymbol{b}$ the coil is positioned around the tip apex and the substrate. Setup $\boldsymbol{b}$ is constructed to investigate Au tips, which are electrochemically coated with a thin layer of Ni. In this way a magnetic field is 
ensured at the position of the magnetostrictive material, since no such field would be propagated by diamagnetic Au.

Investigations of magnetostriction are accomplished by operating the coil during STM measurements. A sinusoidally modulated current $I_{\text {coil }}^{f_{\text {mod }}}$ causes an equally modulated magnetostriction effect of the magnetic tip in use. An illustration of a negative magnetostriction measurement is shown in Figure 4.11.

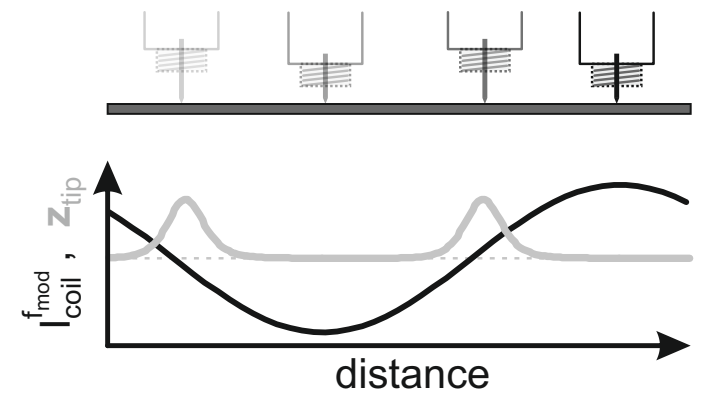

Figure 4.11: Sketch of a scanning tip with a negative magnetostriction effect. During the STM measurement the magnetic field of the coil is modulated by $I_{\text {coil }}^{f_{\text {mod }}}$. Caused by the magnetostriction the STM control readjusts the changing tip-sample separation $\Delta L \rightarrow \Delta d \rightarrow z_{\text {tip }}$ to keep the tunneling current $I_{0}$ constant.

The modulation current $I_{\text {coil }}^{f_{\text {mod }}}$ of the coil at $f_{\text {mod }}$ is recorded simultaneously to STM measurements. By using STM in the constant-current mode and applying a low modulation frequency $f_{\text {mod }}=7.13 \mathrm{~Hz}$ to $I_{\text {coil }}$ far below the operation frequency of the STM feedback control, all changes in tip-sample separation $d$ caused by magnetostriction $\Delta L$ are directly compensated $\rightarrow z_{\text {tip }}$. A magnetostriction measurement such as sketched in Figure 4.11 is only possible if the saturation magnetization is reached at both magnetic field directions, see Figure 4.8. In case of a negative magnetostriction effect the maximum tip length is reached with zero magnetization of the tip at the coercive field strength.

Via the applied current $\left|I_{0 \text {, coil }}\right|$ the resulting magnetic field strength can be calculated for each coil. In such a STM measurement both the modulation of the magnetic field as well as the corresponding magnetostriction effect are detected, since the resulting topographical STM image contains the magnetostrictive information of the magnetic tip. Therefore, the investigated substrate should be a single crystal featuring large, atomically flat terraces to visualize the magnetostriction clearly.

For a clear presentation, where the magnetostriction signal is easily observed in the topographical STM image, the modulation frequency $f_{\text {mod }}$ and the STM scan rate (STM scan line per second, $f_{S T M}$ ) have to be attuned to each other. Using the known STM scan rate the $x$-axis and y-axis can be converted into time scale, which makes comparing different measurements easier.

\section{Coil Magnetic Field}

The magnetic field strength of each coil ( $\operatorname{setup} \boldsymbol{a}$ and setup $\boldsymbol{b})$ is calculated. 
a

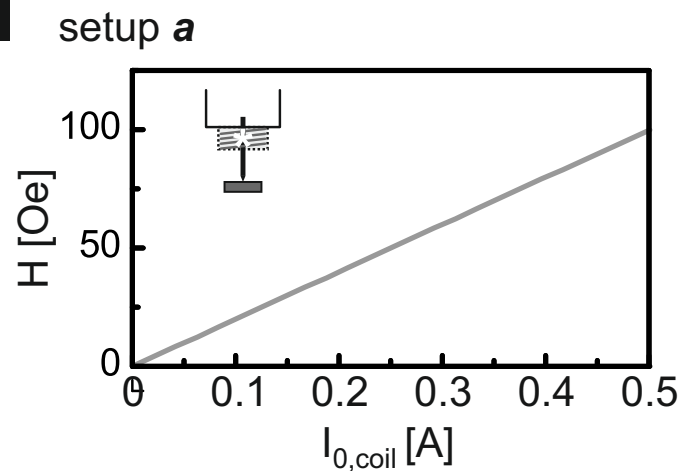

b $\operatorname{setup~} \boldsymbol{b}$

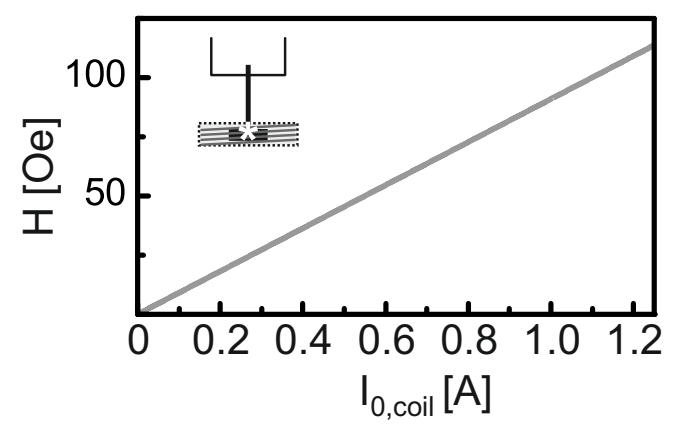

Figure 4.12: Shown is the magnetic field strength of the coil versus the adjusted modulation voltage $U_{m o d}$ for (a) setup $\boldsymbol{a}$. Here the magnetic field strength is calculated for the centre of the coil, where the magnetic tip goes along (marked by the asterisk within the sketch). For (b) setup $\boldsymbol{b}$ the magnetic field strength is calculated for the position of the tip apex (marked by the asterisk within the sketch), where the magnetostrictive material of the NiAu tips is located.

According to Equation (4.14), the magnetic field strength $H$ of setup $\boldsymbol{a}$ is plotted versus the adjusted modulation current $I_{0, \text { coil }}$ in Figure 4.12 (a). Thereby, $H$ is calculated for the center of the coil (marked by the asterisk), where the magnetic STM tip is positioned, and thus its magnetization is modulated. The magnetization of the tip is switched by the coil positioned at the upper end of the tip. It is assumed that the magnetic field is provided by the entirety of the tip from upper part to tip apex [205]. The required modulation current $I_{0, \text { coil }}$ and hence the magnetic field strength should be as low as possible in order to prevent influences on the surface produced by magnetic stray fields from the coil.

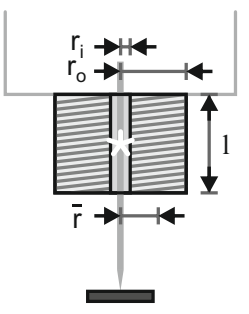

$$
\begin{array}{r}
H_{z, \text { setup } a}=\frac{N \cdot \frac{U_{\text {mod }}}{R}}{2 \sqrt{\bar{r}^{2} \cdot\left(\frac{l}{2}\right)^{2}}} \\
\text { with } R=2.4 \Omega, \\
N=101, \\
l=3.5 \mathrm{~mm}, \\
\bar{r}=\frac{r_{o}-r_{i}}{2}+r_{i}=2.65 \mathrm{~mm} .
\end{array}
$$

The calculated magnetic field strength for setup $\boldsymbol{b}$ is plotted in Figure 4.12(b) versus the adjusted current $I_{0, \text { coil }}$, according to Equation 4.15). Here, the magnetic field strength is calculated for a position inside the coil but only $a=2.5 \mathrm{~mm}$ from the coil edge away. At this position the tip apex is supposed to be in tunneling contact to the substrate during STM magnetostriction measurements. 


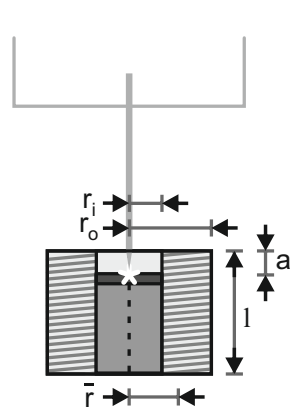

$$
\begin{array}{r}
H_{z, \text { setup } \boldsymbol{b}}=\frac{N \cdot \frac{U_{\text {mod }}}{R}}{2 \cdot l} \cdot\left(\frac{l-a}{\sqrt{\bar{r}^{2}+(l-a)^{2}}}+\frac{a}{\sqrt{\bar{r}^{2}+(a)^{2}}}\right) \\
\text { with } R=2.4 \Omega, \\
N=143, \\
l=10 \mathrm{~mm}, \\
\bar{r}=\frac{r_{o}-r_{i}}{2}+r_{i}=7.75 \mathrm{~mm}, \\
a=2.5 \mathrm{~mm} .
\end{array}
$$

In setup $\boldsymbol{a}$ the influence of magnetic stray fields on an investigated magnetic surface is minimized by the construction. In contrast, in setup $\boldsymbol{b}$ the surface magnetization would be influenced by the same magnetic field strength as the tip apex, since both are positioned inside the coil.
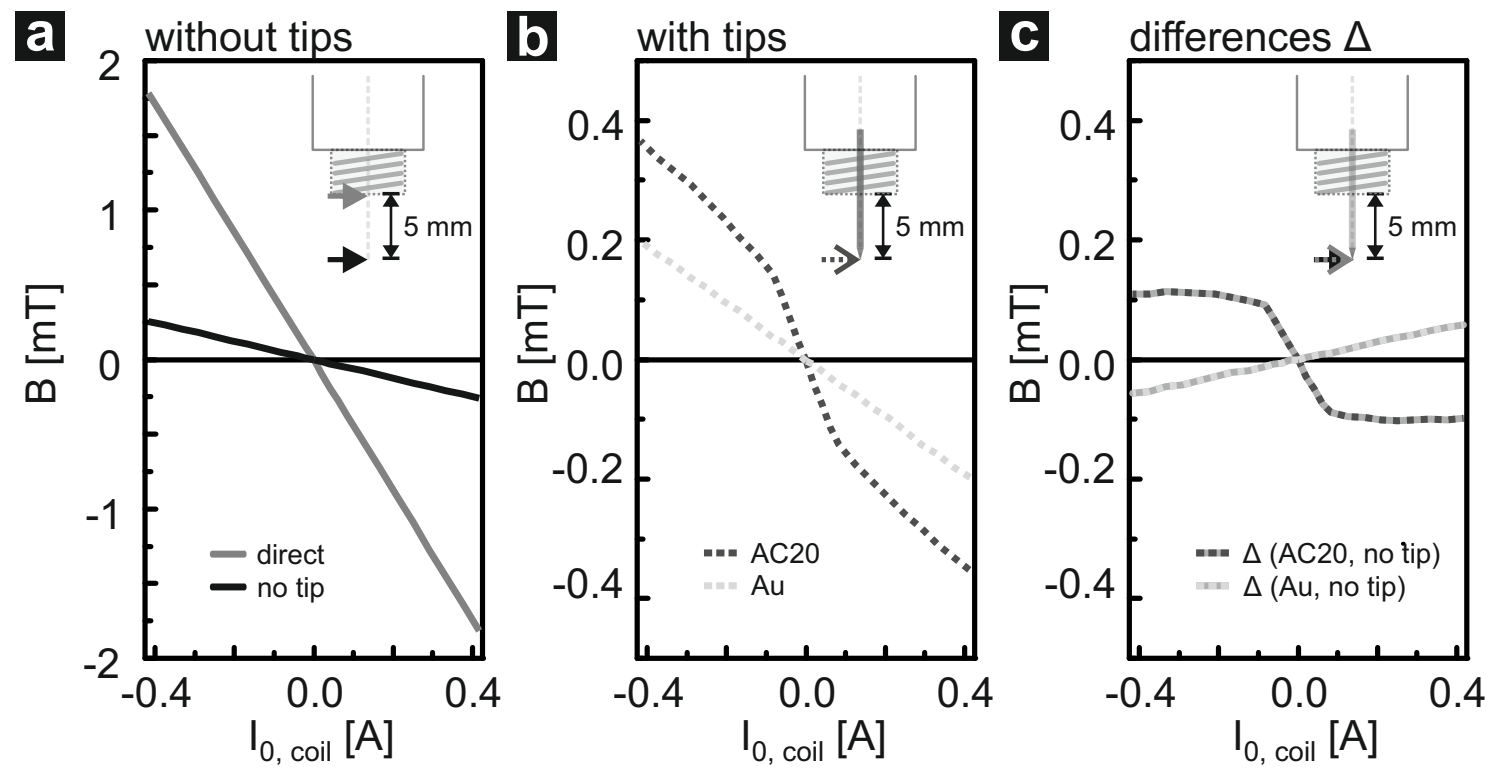

Figure 4.13: Measurements are done with a Hall probe directly located at the illustrated position (a) Magnetic field in dependence of the applied current $I_{0, \text { coil }}$ is measured at the coil cover (gray) an at a distance of $5 \mathrm{~mm}$ (black), where the tip apex would be situated. (b) The magnetic field is measured at the tip apex of an inserted $\mathrm{Au}$ (light gray) or AC20 tip (dark gray), both positioned $5 \mathrm{~mm}$ from the coil cover. (c) The difference between the measurements $\Delta$ (AC20,no tip) reveals a ferromagnetic behavior of $\mathrm{AC} 20$ as well as a diamagnetic behavior of Au by $\Delta$ (Au,no tip).

In Figure 4.13 magnetic fields of setup $\boldsymbol{a}$ are measured for different sceneries using a Hall probe. The values are plotted versus the applied current $I_{0, \text { coil }}$. Ini- 


\section{SP-STM}

tially, for measurements shown in Figure 4.13(a), no tip is mounted on the STM scanner unit. These measurements are either taken directly at the coil cover or at a distance $z=5 \mathrm{~mm}$ where the tip apex would normally be positioned. According to Equation (4.10) the magnetic field strength increases linearly with increasing current $I_{0, \text { coil }}$ at both positions, but decreases with increasing distance $z$ from the coil. Comparative measurements with an inserted Au tip or magnetic AC20 tip are plotted in Figure $4.13(\mathrm{~b})$, each tip was $5 \mathrm{~mm}$ in length and the magnetic field was measured directly at the tip apex. In case of the magnetic AC20 tip a significantly higher magnetic field is observed. This implies, that the magnetic field is promoted by the magnetic AC20 tips contrary to the diamagnetic Au tip. The diamagnetic behavior of an $\mathrm{Au}$ tip is reflected by the difference $\Delta(\mathrm{Au}$, no tip) between the measurements with an Au tip and with no inserted tip, Figure 4.13(c). The typically ferromagnetic behavior of the magnetic AC20 tips is observed by the difference $\Delta$ (AC20, no tip) between the measurements with an AC20 tip and with no inserted tip.

These observations underline that magnetostriction measurements of $\mathrm{Ni}$ coated Au-tips have to be investigated by setup $\boldsymbol{b}$, since diamagnetic Au tips reduce the actual magnetic field at the tip apex.

\subsubsection{Investigation of Magnetostriction}

As mentioned before, sp-STM requires magnetic STM-tips with vanishing magnetostriction and low coercivity. STM tips made from AC20, pure Ni, and Ni coated Au-tips $(\mathrm{NiAu})$ were investigated.

The magnetic properties of the different tip materials are of great importance for sp-STM. Therefore, all tip materials are initially characterized by SQUID measurements.

Furthermore, magnetostriction of the different tip materials is investigated by the introduced STM setups $\boldsymbol{a}$ and $\boldsymbol{b}$.

\section{SQUID Investigation of Different Tip Materials}

SQUID measurements for $\mathrm{AC} 20$, pure $\mathrm{Ni}$, and electrochemically deposited $\mathrm{Ni}$ on $\mathrm{Au}$ with $\sim 350 \mathrm{~nm}, 700 \mathrm{~nm}$ and $1400 \mathrm{~nm}$ layer thickness are shown in Figure 4.14 . $\mathrm{Ni}$ coated $\mathrm{Au}$ tips are fabricated by electrochemically depositing Ni from a Ni-rich bath (see A.2 for further information) within an electrochemical setup configuration of three electrodes ( $\mathrm{SCE}$ as $R E$, pure $\mathrm{Ni}$ wire as $C E$, and the $\mathrm{Au}$ tip as $W E$ ). The deposited Ni thickness is estimated by measuring the charge transfer at the submerged Au tip surface area.

As expected, SQUID measurements for all samples show ferromagnetic hysteresis loops. For pure Ni and AC20 the measured magnetization is normalized to the volume of the investigated material. 

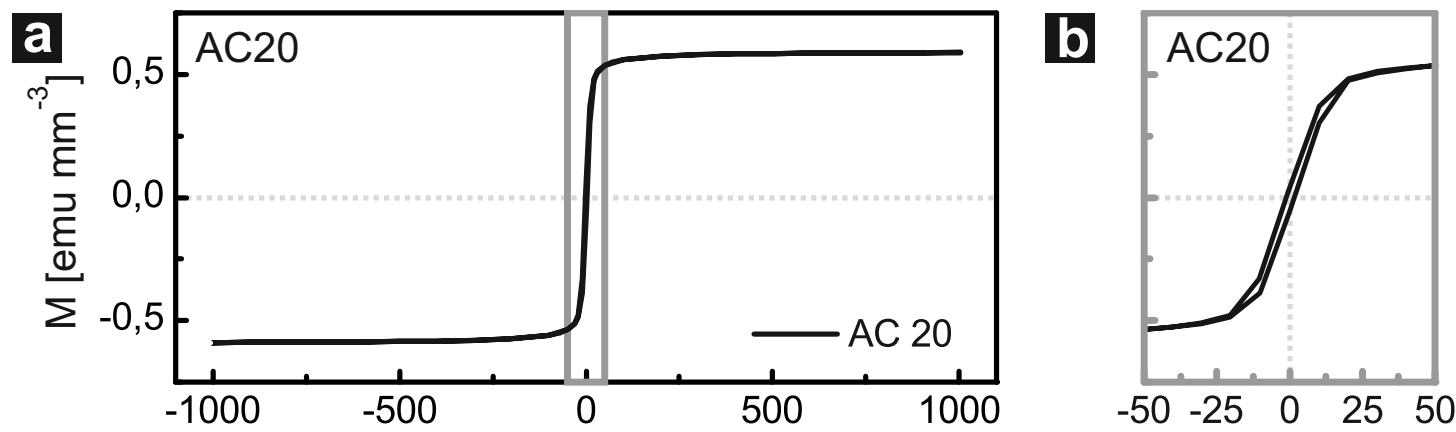

$\mathrm{H}[\mathrm{Oe}]$

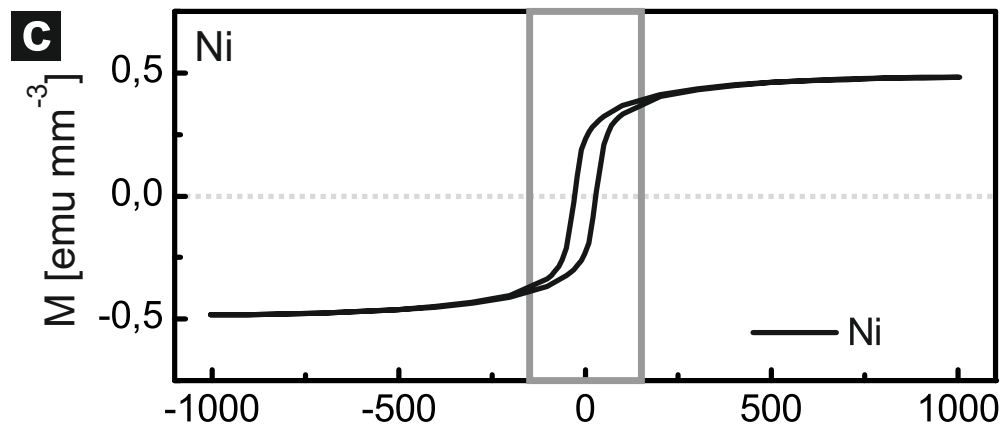

d $\mathrm{H}[\mathrm{Oe}]$

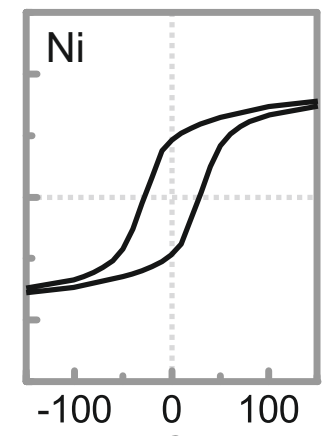

$\mathrm{H}[\mathrm{Oe}]$
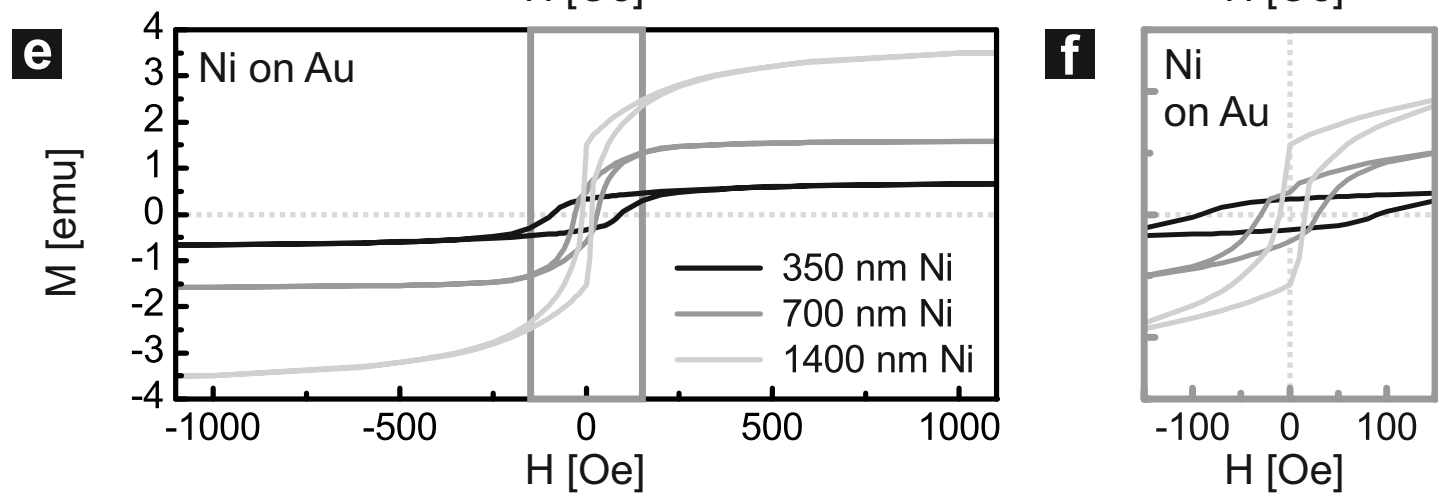

Figure 4.14: SQUID measurements of the hysteresis loops of (a) $\mathrm{Co}_{68.2} \mathrm{Fe}_{4.3} \mathrm{Si}_{12.5} \mathrm{~B}_{15}$ (AC20) wire, (c) pure $\mathrm{Ni}$ wire, and (e) deposited $\mathrm{Ni}$ on an $\mathrm{Au}$ wire with various coating thicknesses. (b, $\mathbf{d}$ and $\mathbf{f}$ ) Close-ups of the hysteresis loops in a lower range of the magnetic field strength $H$. 


\section{SP-STM}

In case of NiAu350, NiAu700, and NiAu1400, the magnetization is presented as measured without normalization to magnetic volume, since the actual volume of the deposited material can only be estimated rather poorly. Therefore, the measured saturation magnetization of NiAu samples increases nearly linearly with increasing layer thickness, while the width of the hysteresis loop decreases.

Compared to all others, AC20 shows a nearly rectangular hysteresis loop, which indicates a 'soft' ferromagnet. The change from one magnetization direction to the other along the $z$-axis occurs within a small range and the saturation magnetization of the AC20 sample is already reached at $H<50$ Oe.

The coercive field strength $H_{c}$ of AC20 is in the range of 1 Oe and thus significantly lower than the coercive field strength $H_{c}$ of pure $\mathrm{Ni}$ with $35 \mathrm{Oe}$ and of the deposited $\mathrm{Ni}$ on $\mathrm{Au}$ ranging between 90 Oe and 20 Oe. Attention should be given to a significantly higher coercivity which is observed for thinner Ni films. The coercivity can be increased for $\mathrm{Ni}$ and NiAu tips by thermal treatment (not shown). But, alloy formation and oxidation degrade the quality of such tips.

\begin{tabular}{cccccc}
\hline & $\mathrm{AC} 20$ & $\mathrm{Ni}$ & $\mathrm{NiAu} 350$ & $\mathrm{NiAu} 700$ & $\mathrm{NiAu} 1400$ \\
\hline$H_{C}$ & $1 \mathrm{Oe}$ & $28 \mathrm{Oe}$ & $90 \mathrm{Oe}$ & $29 \mathrm{Oe}$ & $12 \mathrm{Oe}$ \\
\hline
\end{tabular}

Table 4.3: Coercivity of the investigated tip materials measured by SQUID.

\section{Magnetostriction Measurements}

All investigated magnetic STM tips are made by electrochemical etching within a lamella of acid, as illustrated in Section 3.2.2. NiAu tips are additionally electrochemically treated.

A typical magnetostriction measurement of an AC20 tip (setup $\boldsymbol{a}$ ), is shown in Figure 4.15. The magnetic field is sinusoidally modulated and measured indirectly by recording $\left(U_{0, \text { coil }}^{f_{\text {mod }}}=I_{0, \text { coil }}^{f_{\text {mod }}} \cdot R_{\text {coil }}\right)$ at the coil via the STM's auxiliary channel. Due to the low applied modulation frequency $f_{\text {mod }}=7.13 \mathrm{~Hz}$ and a scan rate of $f_{S T M}=1.42 \mathrm{~Hz}$ this magnetic field modulation becomes visible as a regular pattern, as observed in Figure 4.15(a). Besides topographical information of the $\mathrm{Au}(111)$ surface displaying some terraces separated by monatomic steps, an additional modulation is observed within the STM image caused by the magnetostrictive AC20 tip, see Figure 4.15(b). Two corresponding profiles of the field modulation and the topography are displayed (dashed arrows) for comparison, plotted in Figure 4.15(c $\& d)$. Besides the magnetostriction effect of the tip, an $\mathrm{Au}(111)$ step edge with $a_{A u}=2.4 \AA$ is observed. Both corresponding profiles underline that AC20 magnetostriction occurs at $f_{\lambda}=2 f_{\text {mod }}$. For AC20 the zero-crossing is nearly equal to the low coercive field strength as measured by SQUID (Figure 4.14(a)). Peaks corresponding to the zero-crossing of the field modulation occur within the topographical 


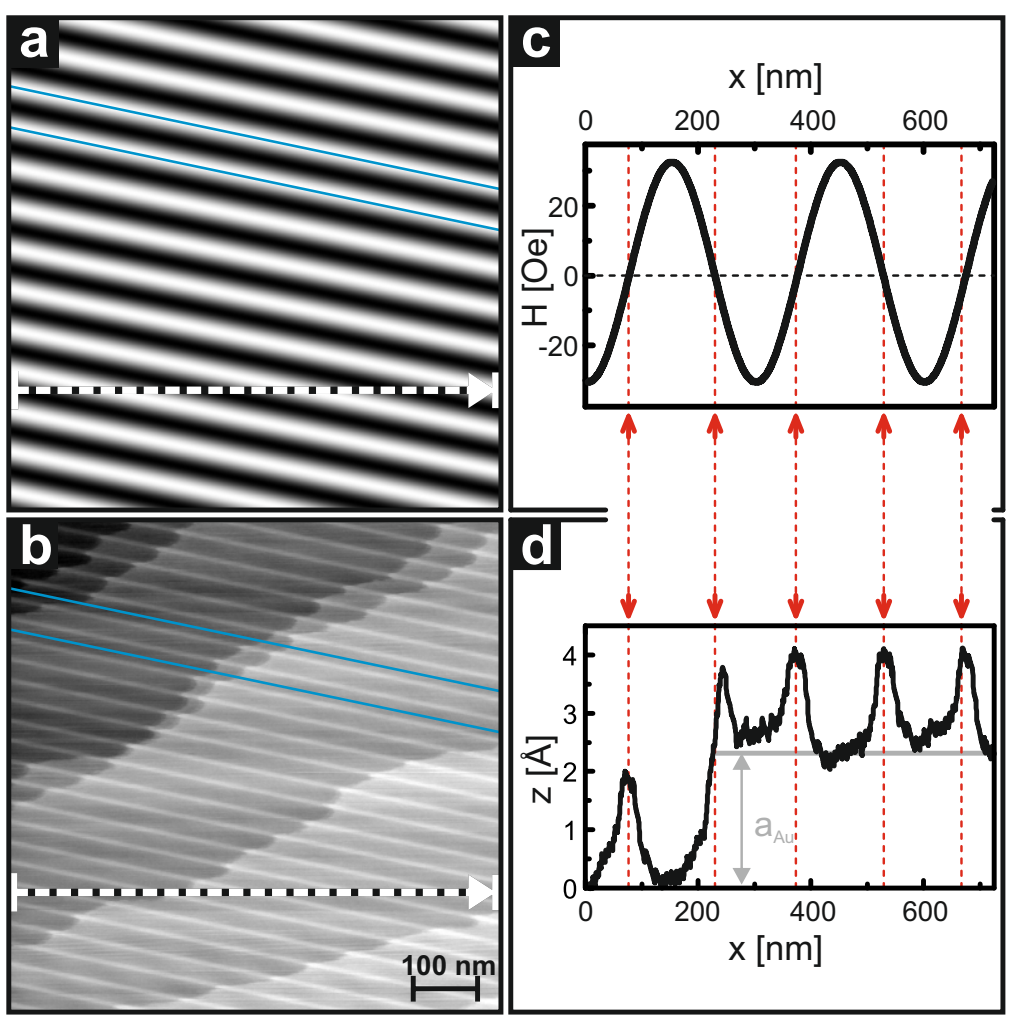

Figure 4.15: (a) The sinusoidal modulation of the coil is equal to the modulation of the magnetic field and is recorded by the auxiliary input of the STM setup. A regular pattern is observed caused by the low modulation frequency $f_{\bmod }=7.13 \mathrm{~Hz}$ and the STM scan rate $f_{\mathrm{STM}}=1.42 \mathrm{~Hz}$. (b) The corresponding topographic STM image also shows the regular modulated pattern in addition to the $\mathrm{Au}(111)$ terraces. Two blue lines highlight the correlation. The dashed arrows indicate profiles which are shown in (c \& d). (c) The magnetic field is clearly sinusoidally modulated with a magnitude of 33 Oe. The zero-crossing is similar to the coercive field strength of AC20 (see SQUID measurement). Therefore, at this points the negative magnetostrictive tip has its maximum length and the STM control has to retract from the surface to keep $I_{0}$ constant. (d) Peaks corresponding to the zero-crossing occur within the topographical profile, marked by the red arrows. 


\section{SP-STM}

profile, which are marked by red arrows in Figure 4.15 (d). At these points, the negative magnetostrictive tip has its maximum length and the STM control has to retract the scanning piezo from the $\mathrm{Au}(111)$ surface to keep the tunneling current $I_{0}$ constant.

Compared to the simulated magnetostriction effect in Figures 4.8 and 4.9 and supported by the SQUID measurement, Figure 4.14(a), a magnetic field of 33 Oe is sufficient to switch the AC20 tip magnetization and to cause a saturated magnetostriction of the AC20 tip.
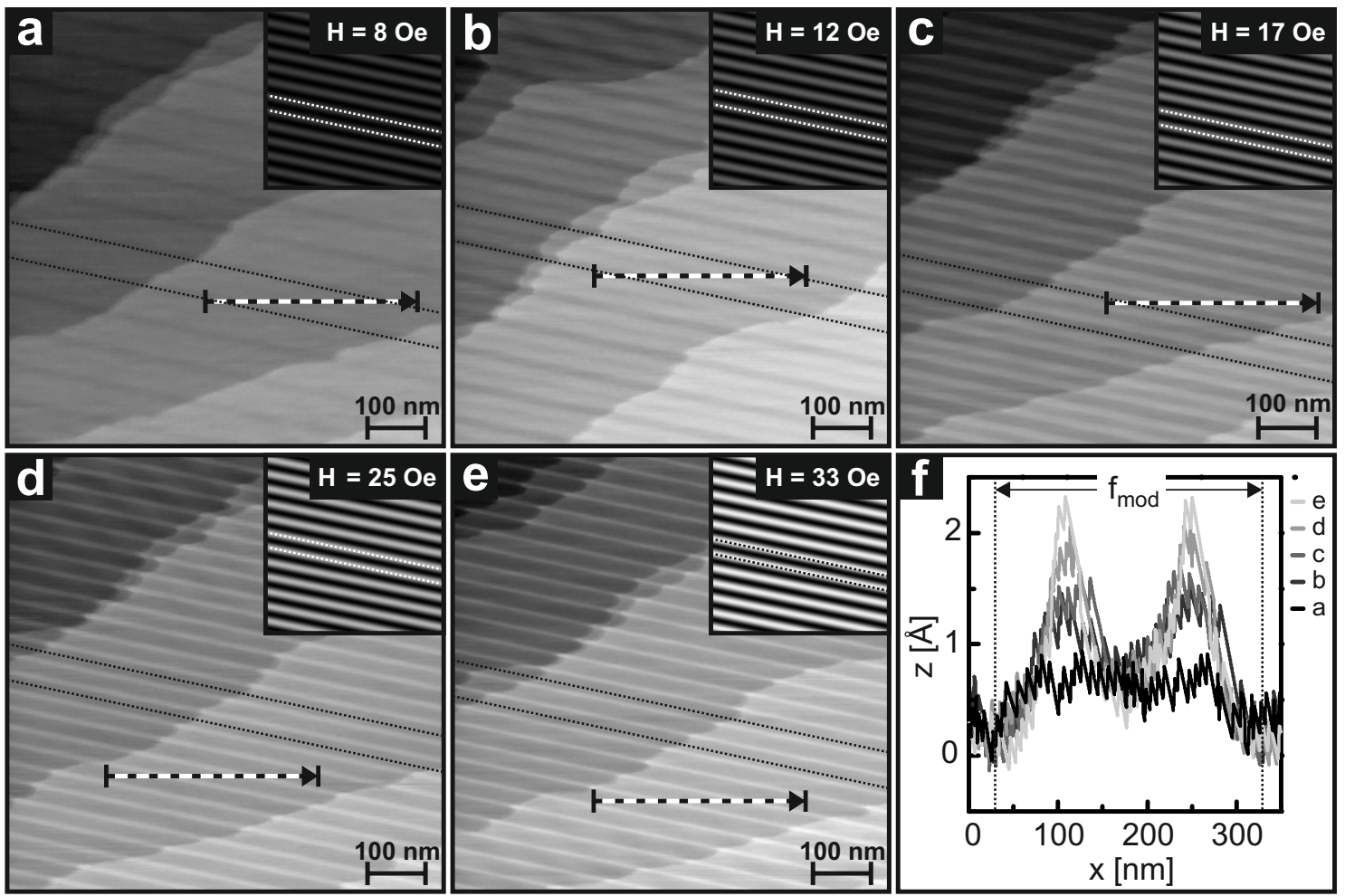

Figure 4.16: $I_{0}=4 \mathrm{nA} ; E_{\mathrm{WE}}=-219 \mathrm{mV}(\mathrm{Au}(111)) ; E_{\text {tip }}=-374 \mathrm{mV}(\mathrm{AC} 20) ;$ $f_{\text {mod }}=7.13 \mathrm{~Hz} ; f_{\mathrm{STM}}=1.42 \mathrm{~Hz}$ (STM scan rate). (a)-(e) Series of STM measurements: the magnetostriction of an AC20 tip is shown for increasing magnetic fields. These measurements are taken with setup $\boldsymbol{a}$. The insets show the modulation of the coil. Correlated to the topographical image, two dashed lines exemplarily mark maximum field strength. Due to the higher magnetic fields the magnetostriction signal develops from a modulation at $1 f_{\text {mod }}$ (a) to a signal at $2 f_{\text {mod }}(\mathrm{e})$. In (f) all magnitudes of the magnetostriction from figure (a) to (e) are summarized for comparison.

The transition to a signal at $2 f_{\text {mod }}$ is observable.

In Figure 4.16(a-e) the development of the AC20 magnetostriction is investigated by a sequence of STM measurements of the same $\mathrm{Au}(111)$ surface area at increasing 
magnetic field. The topographical STM images with inserted corresponding modulation of the magnetic field are shown, their correlation being highlighted by two lines at maximum field strength. Superimposed onto the topographical information the magnetostriction effect of the AC20 tip is clearly visible. Cross sections along the $x$-axis are taken (white dashed lines) and summarized in Figure 4.16(f) for a better comparison between the different magnetostrictive magnitudes. The frequency of all magnitudes corresponds to the sinusoidal modulation of the coil. By applying a higher magnetic field the frequency of the magnetostriction becomes twice the modulation frequency $f_{\lambda} \rightarrow 2 f_{\text {mod }}$ and the magnitude decreases. This becomes obvious comparing profiles (a) and (e). With a magnitude of $\Delta L \approx 2 \AA$ the total saturated magnetostriction of the AC20 tip is of the same order as a monatomic $\mathrm{Au}(111)$ step. Since the profile (d) at $25 \mathrm{Oe}$ is similar to (e) at $33 \mathrm{Oe}$, a magnetic field strength of 33 Oe seems to be high enough to switch the tip magnetization of the entire tip. This behavior is reasonable compared to the SQUID measurement of AC20, Figure 4.14(b).

Further magnetostriction measurements are presented in the same way as in Figure 4.16(f), the magnitude of magnetostriction is plotted versus the distance $(x$ axis). Thereby, the magnetostriction of NiAu tips is measured by STM setup $\boldsymbol{b}$, where the coil is located around the substrate and thus around the tip apex. In Figure 4.17(a-c) the magnetostriction investigations for a NiAu175, a NiAu350, and a NiAu700 tip are plotted, respectively. The magnetostriction measurement for AC20 is shown in Figure 4.17(d) for comparison. Although the magnitude of the magnetostriction of the NiAu175 tip is in the range of the previously investigated AC20 tip, much larger magnetic field strengths are required to observe a magnetostriction at $2 f_{\text {mod }}$. With increasing thickness of the deposited Ni layer the required magnetic field strength decreases, but on the other hand the magnitude of the magnetostriction increases up to about $20 \AA$ for the NiAu700 tip. With regard to the SQUID measurements the observed behavior is reasonable, since the thinner Ni layers show a higher coercivity. The thicker coated NiAu tips show a larger magnetostriction caused by the thickness of the magnetostrictive layer

Magnetostriction of a pure $\mathrm{Ni}$ tip is measured with setup $\boldsymbol{a}$ and is shown in Figure 4.18. The observed elongation of the pure Ni tip amounts to about $800 \AA$ and thus much higher than of all other investigated tip materials. The setup in this case however is at its limit, since the actual magnetostrictive material length is in the range of $15 \mathrm{~mm}$ and the magnetostriction coefficient is very large. Thus, magnetostriction measurements at higher magnetic fields in order to reach the saturation magnetostriction are not possible. However, such kind of measurements are not useful either, since from the already observed large magnetostriction it is clear that pure Ni tips are not suitable for sp-STM measurements.

Comparable magnetostriction measurements with $\mathrm{Au}$ tips at a low modulation frequency show neither a modulation in height with $1 f_{\text {mod }}$ nor with $2 f_{\text {mod }}$, therefore 


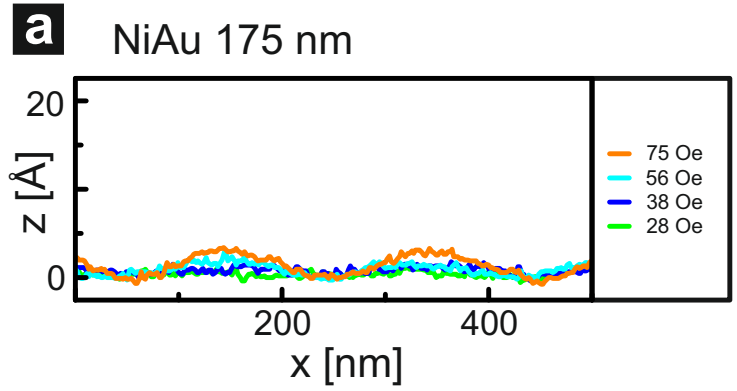

C NiAu $700 \mathrm{~nm}$
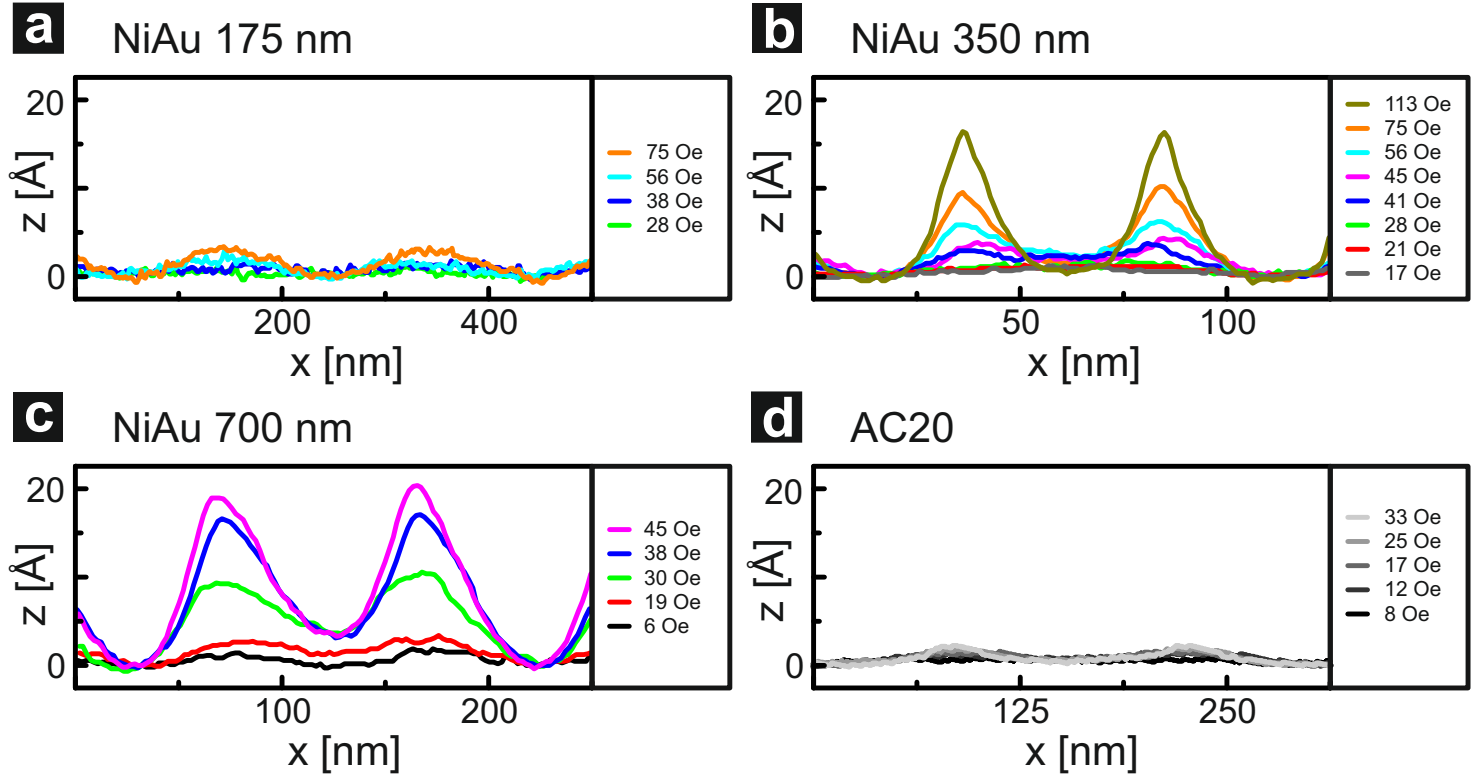

d $\mathrm{AC} 20$

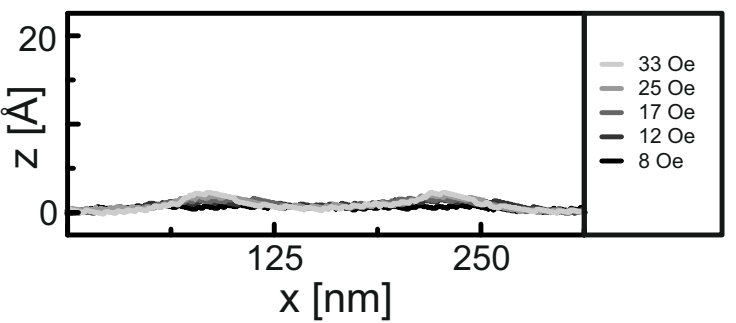

Figure 4.17: (a-c) Measured with setup $\boldsymbol{b}$. For all investigated NiAu tips a clear increase of the $2 f_{\text {mod }}$ signal is observed with higher magnetic field strengths. With increasing nominal $\mathrm{Ni}$ thickness a lower magnetic field strength is required to observe a signal with $2 f_{\text {mod }}$. (d) The magnetostriction measurement of the AC20 tip is shown for comparison.

Figure 4.18: The magnetostriction of a Ni tip is measured with setup $\boldsymbol{a}$. For comparison with measurements at different modulation frequencies, the magnitude is plotted versus $x \cdot f_{\text {mod }}$. The magnitude of the magnetostrictive tip increases up to $800 \AA$ with increasing magnetic field strengths.

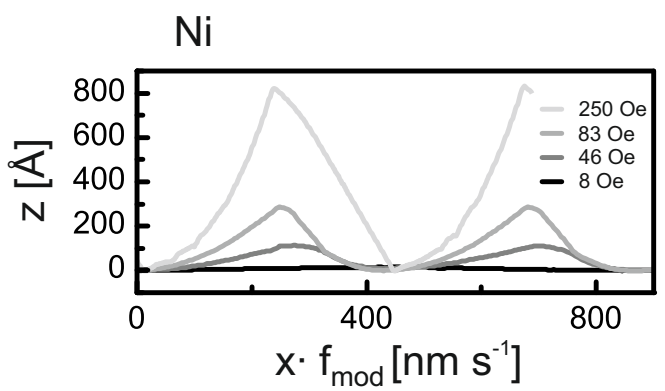

effects of the STM setups on the STM magnetostriction measurement can be ruled out.

Magnetic sp-STM tips are exclusively made of an AC20 wire, which is $125 \mu \mathrm{m}$ in diameter, since it is a soft ferromagnetic and amorphous alloy [40] with a low negative magnetostriction $-4 \cdot 10^{-8}$ and a low coercivity in the range of 1 Oe.

Since the STM tip holder at the scanner unit is designed for tips with $250 \mathrm{\mu m}$ in diameter, magnetic AC20 tips for sp-STM consist of two parts, glued together by conductive silver paint. The upper part, which is mounted on the scanner unit, is made of pure Au wire. The lower part, near to the investigated sample surface, is 
an electrochemically etched AC20 tip made of the thinner magnetic wire.

The electrochemical etching of these sp-STM tips and all further preparation steps have been detailed and illustrated in Section 3.2.2. For AC20 tip preparation an etching voltage of $2.4 \mathrm{~V}$ is used. It is important to attach a pull down weight to the AC20 wire, because otherwise no drop-off would occur. This would result in continued etching and therefore dull tips.

\subsubsection{Magnetostriction at High Modulation Frequency}

Sp-STM investigations at the solid/liquid interface on magnetic samples are accomplished with setup $\boldsymbol{a}$ using high modulation frequencies above the cut-off-frequency of the STM setup $\left(f_{\text {mod }} \gg f_{\text {cut-off }} \approx 20 \mathrm{kHz}\right)$. The modulation of the tip-surface distance $d$, caused by magnetostrictive effects, generates modulated components of the tunneling current at $1 f_{\text {mod }}$ and $2 f_{\text {mod }}$. A suitable tip made of AC20 reduces the magnetostrictively modulated component of the tunneling current to a $2 f_{\text {mod }}$ component, which does not affect sp-STM measurements. Because of its magnetic properties AC20 is commonly used as a material for tips in sp-STM at solid/vacuum interfaces [46, 168, 202, 203].

An example for a STM investigation on an $\mathrm{Au}(111)$ surface using a magnetic AC20 tip is shown in Figure 4.19 during modulation with $f_{\text {mod }}$. During this measurement the magnetic field of setup $\boldsymbol{a}$ is modulated at a frequency of $f_{\text {mod }}=52.137 \mathrm{kHz}$ above the cut-off-frequency of the STM. The resulting topography $z(x, y)$ Figure $4.19(\mathrm{a})$ ), the averaged tunneling current $\bar{I}(x, y)$ (Figure $4.19(\mathrm{~b})$ ), and the observed modulated component of the tunneling current at $f_{\bmod }$ are recorded (Figure $4.19(\mathrm{c}))$. Both signals $\bar{I}(x, y)$ and $z(x, y)$ occurs in high quality caused by the vanishing magnetostriction of the modulated magnetic AC20 tip, the herringbone structured $\mathrm{Au}(111)$ surface is also observable. $\bar{I}(x, y)$ is constant during the entire sp-STM measurement. Only at $\mathrm{Au}(111)$ step edges peak-like increasing or decreasing signals are observed. This is quite as expected, a change in tip-sample distance $d$ at step edges has to be recognized by the STM feedback loop, which adjusts the separation. Since a steeply increasing signal is a superposition of signals at higher frequencies, an abrupt rising signal in $\bar{I}$ at step edges is also observed by lock-in technique at $f_{\text {mod }}$. Thereby, step edges without spin-polarized origin are displayed in the lock-in signal $\Delta I^{f_{\text {mod }}}$ alongside the constant signal above $\mathrm{Au}(111)$ terraces. These unavoidable artifacts are observed in $\Delta I^{f_{\text {mod }}}$ at every abrupt change in height, therefore flat sample areas are preferred for investigation.

It is important to note that the scan rate of the STM should be adjusted to about $0.9 \mathrm{~Hz}$ in order to obtain a lateral resolution in $\Delta I^{f_{\text {mod }}}$ comparable to the topographical STM image. This is observed in Figure 4.19(c) at step edges, which are extended compared to the corresponding observation in Figure 4.19(a). Thereby, a slower scan rate combined with a higher time constant minimizes the signal noise. 


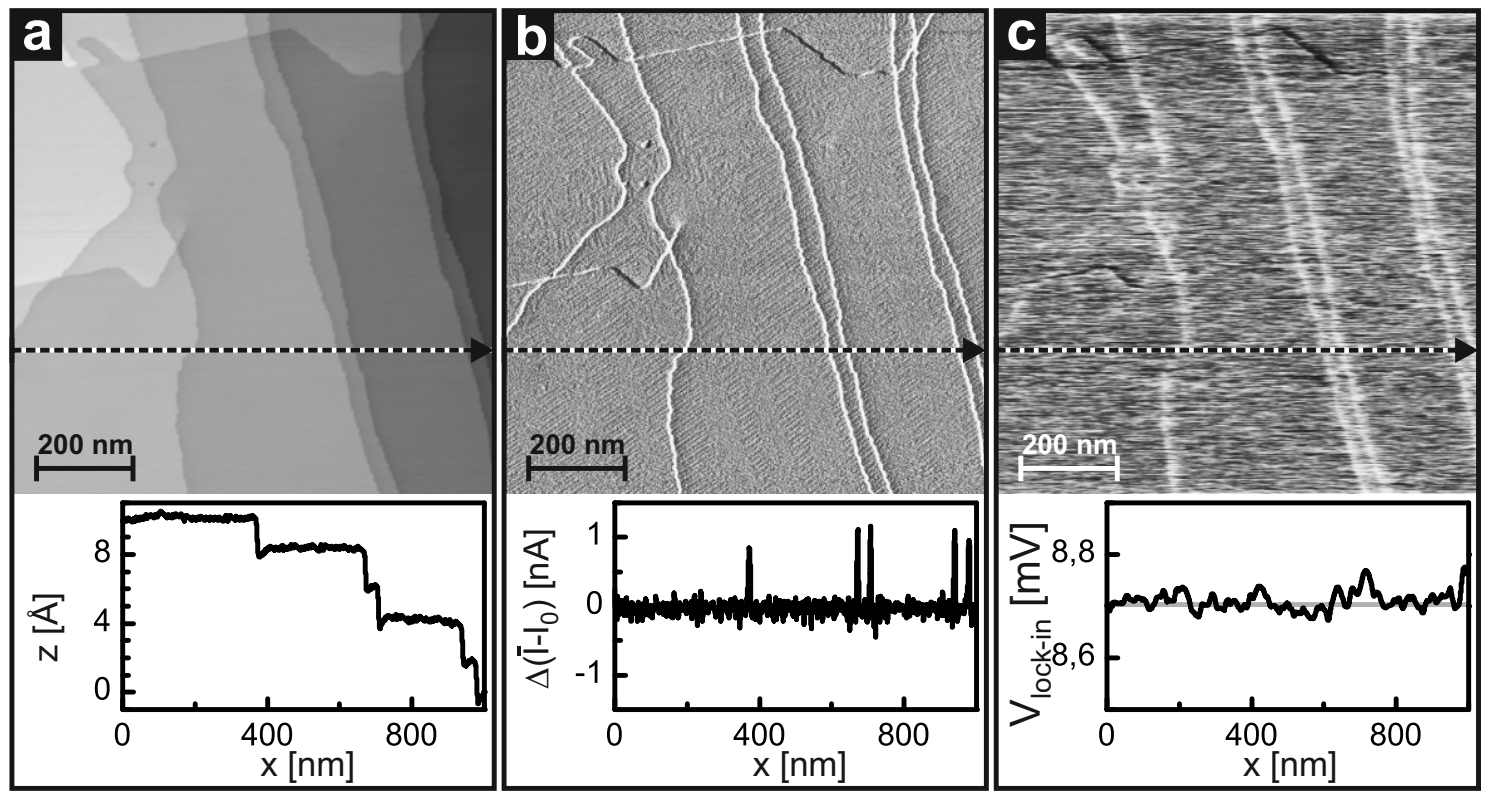

Figure 4.19: $\mathrm{STM}$ measurement on $\mathrm{Au}(111)$ using an AC20 tip during modulation with $f_{\text {mod }}$ above the cut-off-frequency of the STM. $I_{0}=1.5 \mathrm{nA} E_{\mathrm{WE}}=-715 \mathrm{mV}$ $(\mathrm{Au}(111)) ; E_{\mathrm{tip}, \mathrm{AC} 20}=-700 \mathrm{mV}(\mathrm{AC} 20) ; f_{\mathrm{mod}}=52.137 \mathrm{kHz} ; f_{\mathrm{STM}}=1.3 \mathrm{~Hz}(\mathrm{STM}$ scan rate). (a) Topography $z_{\text {tip }}(x, y)$. $\mathrm{Au}(111)$ terraces and step edges are clearly observed. (b) Constant-current $\bar{I}(x, y)$ normalized to the setpoint $I_{0} . \sqrt{3} \times 22$ reconstructions of the $\mathrm{Au}(111)$ surface are observed and spikes in the tunneling current can be attributed to step edges. (c) Lock-in signal at $f_{\text {mod }}$ with time constant $1 \mathrm{~ms}$. At step edges signal peaks are observed corresponding to the tunneling current signal at higher frequencies. To reduce noise in signal as well as to improve resolution, the time constant of the lock-in should be set to $3 \mathrm{~ms}$, and thus $f_{\mathrm{STM}}$ should be adjusted to around $0.9 \mathrm{~Hz}$.

For further measurements the time constant of the lock-in is adjusted to $3 \mathrm{~ms}$.

Any signal caused by magnetostriction at high modulation frequencies persists throughout the whole STM measurement as long as the coil/magnetic field is in operation. Therefore, the tip-surface distance $d$ and the time integrated tunneling current $\bar{I}$ are adjusted to a mean position by the STM in order to keep the tunneling current constant at the setpoint value $I_{0}$. However, this effect cannot account for any contrast between a magnetic and a non-magnetic surface. 


\subsection{Sp-STM on Co/Au(111) at the Solid/Liquid Interface}

Sp-STM at the solid/liquid interface provides the opportunity to investigate magnetism of extended $\mathrm{Co}(0001)$ islands on $\mathrm{Au}(111)$. Electrochemical Co deposition and the resulting morphology of deposited $\mathrm{Co}$ on $\mathrm{Au}(111)$ were already discussed in Sections 2.4 and 3.3. AC20 tips are used exclusively in the lower potential range around $-370 \mathrm{mV}$ with respect to the SHE, since in higher potentials they would react electrochemically and be destroyed. Due to hydrogen evolution it is possible that a higher faradaic current arises at the tip. Therefore, it is very important to keep the bare tip surface exposed to the electrolyte as small as possible.

Initially the magnetic behaviors of Co bulk and thin Co films are summarized. Furthermore, sp-STM investigations on the model system $\mathrm{Co} / \mathrm{Au}(111)$ at the solid/liquid interface are shown. These observations are compared to sp-STM control experiments, where a common STM Au tip is utilized instead of a ferromagnetic AC20 tip.

\subsubsection{Magnetic Behavior of $\mathrm{Co} / \mathrm{Au}(111)$}

The easy axis of magnetization for a hcp Co single crystals points along its $\langle 0001\rangle$ direction, as illustrate in Figure 4.20(a). However, at the (0001) surface the magnetization is directed in-plane, perpendicular to the surface normal $\vec{a}$. The evolving magnetic domains are separated by Bloch walls which entail a localized out-of-plane rotation of the magnetization axis [46, 185, 192. Such a closure domain pattern at the $\mathrm{Co}(0001)$ surface gives the impression of fractal structures observable by scanning electron microscopy with polarization analysis [192] and sp-STM in UHV [46].

In contrast, thin films of Co grown on $\mathrm{Au}(111)$ show a rotation of the easy magnetization axis parallel to the surface normal $\vec{a}$ (out-of-plane) [2, 97, 135, 139, 150]. This magnetic behavior of Co thin films is a consequence of the magnetocrystalline anisotropy [184, 195]. A detailed introduction into this topic of magnetocrystalline anisotropy as well as further information can be found elsewhere [35, 69, 185].

The orientation of the easy magnetization axis of Co films from out-of-plane $\vec{M}_{\|}$ to in-plane direction $\vec{M}_{\perp}$ occurs with increasing layer thickness. It has been reported to occur at room temperature around 1.5 ML Co coverage for electrodeposited Co 2] and around 4.3 ML Co coverage for evaporated Co [97, 135, 139, 150]. The correlation between magnetization and nominal coverage is problematic, since the magnetization $\vec{M}$ of Co in this ultralow coverage regime is strongly correlated to the growth process and the morphology. Differing results in previous literature demonstrate this problem.

However, magnetic properties of structures prepared at the solid/liquid interface 


\section{SP-STM}

a

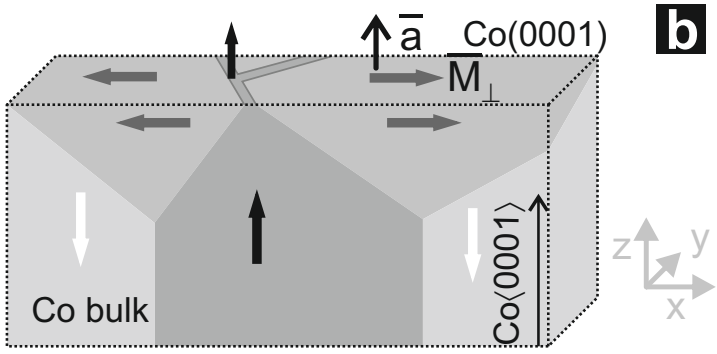

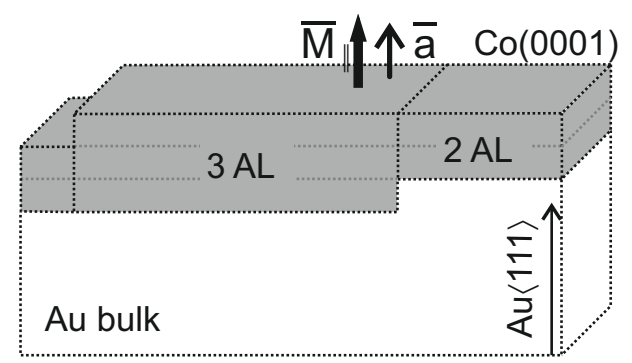

Figure 4.20: (a) Model of a hcp Co single crystal in cross section with $\mathrm{Co}\langle 0001\rangle$ direction parallel to the z-axis, where the easy axis of magnetization $\vec{M}=\vec{M}_{\|}$is arranged along. At the $\mathrm{Co}(0001)$ surface magnetization is in-plane $\vec{M}=\vec{M}_{\perp}$, perpendicular to the surface normal $\vec{a}$. (b) In contrast, thin films of Co grown on $\mathrm{Au}(111)$ show out-of plane magnetization $\vec{M}=\vec{M}_{\|}$at the surface, parallel to $\vec{a}$. A spin reorientation transition from out-of-plane to in-plane magnetization $\vec{M}_{\|} \rightarrow \vec{M}_{\perp}$ at the $\mathrm{Co}(0001)$ surface occurs at higher Co layer thickness.

and in UHV can be investigated ex-situ, utilizing capping layers to protect the magnetic structure from degradation [28, 29, 34, 79, 80, 140, 149, 187, 206]. It is unsuitable to compare those magnetization investigations to uncapped samples measured in-situ, since capping layers cause additional interface effects influencing the magnetic behavior [195].

These in-situ investigations at the liquid/solid interface are accomplished by integral techniques like the magneto-optical Kerr effect (MOKE) [2, 146, 161, 162] with a resolution of about $400 \mathrm{~nm}$ or alternating gradient field magnetometry (AGFM), which gives integrated results of the sample [5, 33, 73]. Measurements of the magnetization of individual structures have been achieved so far only at the solid/vacuum interface. Locally resolved measurements at the liquid/solid interface are required to investigate magnetization of structures with well-defined height and morphology.

\subsubsection{Experiment}

A surface area is chosen for sp-STM investigation covered up to $50 \%$ with partially connected $\mathrm{Co}$ islands on adjacent $\mathrm{Au}(111)$ terraces. These extended Co island are up to 4 atomic layers (AL) high. Thus, a mixed surface with ferromagnetic and diamagnetic areas is prepared where one expects out-of-plane magnetization $\vec{M}_{\|}$at Co sites and no magnetization at pristine $\mathrm{Au}(111)$.

In Figure 4.21(a) such an area under investigation is shown in a the topographical image of a sp-STM measurement. Exemplarily, two selected topographic profiles are indicated and plotted in Figure 4.21(b). The upper profile (gray) is taken across monatomic $\mathrm{Au} / \mathrm{Au}$ step edges each $(2.4 \pm 0.2) \AA$ in height, which act as reference. The lower profile (black) crosses over a $\mathrm{Co} / \mathrm{Au}$ step edge of $3 \mathrm{AL} \widehat{=}(5.3 \pm 0.3) \AA$ 

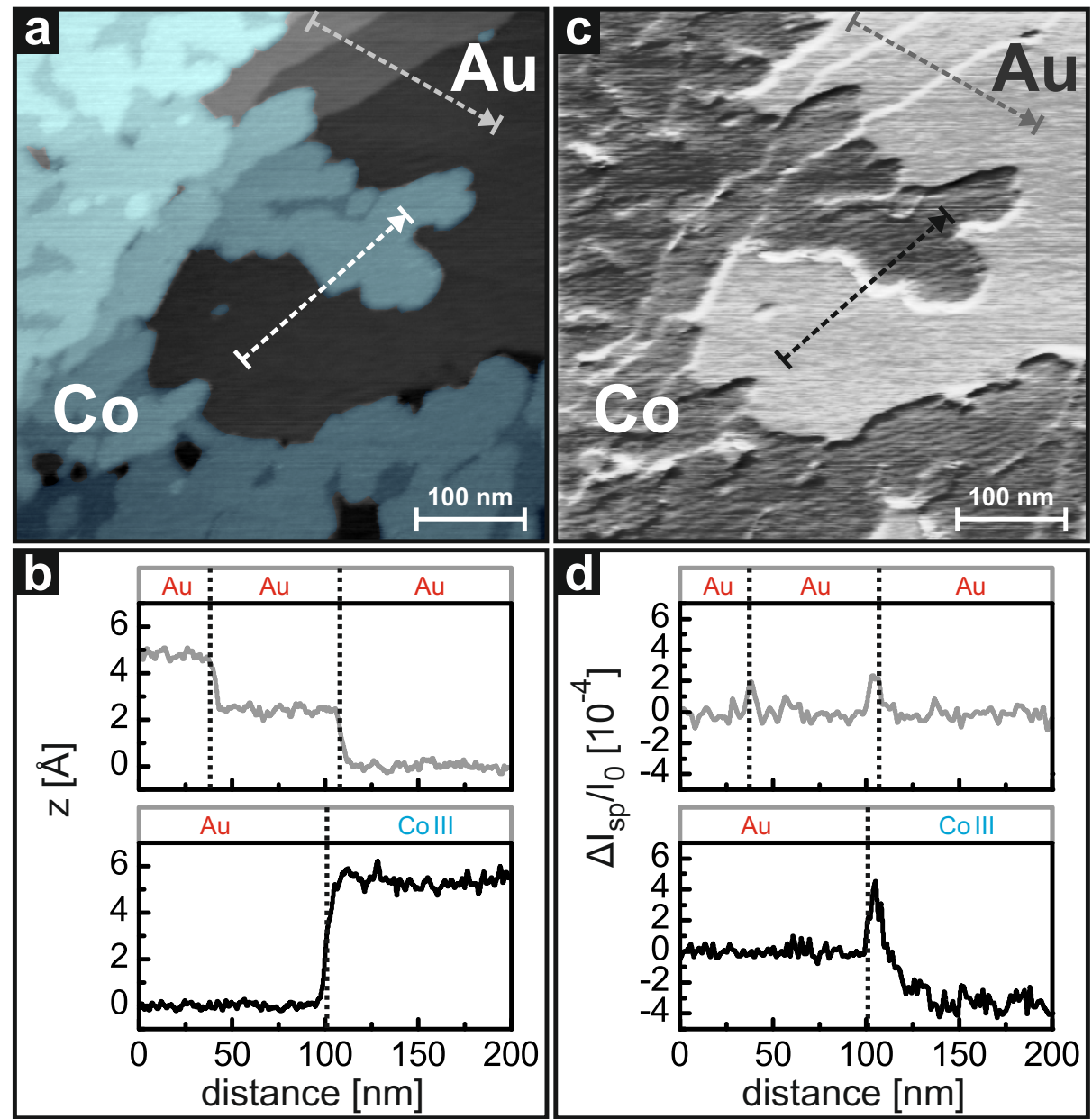

Figure 4.21: Sp-STM of $\mathrm{Co} / \mathrm{Au}(111): I_{0}=6 \mathrm{nA} ; E_{\mathrm{WE}}=-351 \mathrm{mV} ; E_{\mathrm{tip}, \mathrm{AC} 20}=$ $-369 \mathrm{mV} ; f_{\text {mod }}=59.7 \mathrm{kHz}$. (a) Topography of deposited Co (blue), up to $4 \mathrm{AL}$ high on $\mathrm{Au}(111)$. (b) Profiles along the dashed arrows shown in (a) of single atomic $\mathrm{Au} / \mathrm{Au}$ step edges, each $(2.4 \pm 0.2) \AA$, and a $\mathrm{Co} / \mathrm{Au}$ step edge of $3 \mathrm{AL} \widehat{=}(5.3 \pm 0.3) \AA$. (c) Simultaneously measured spin-polarized component of the tunneling current at $f_{\bmod }$ : $\Delta I_{s p}^{f_{\text {mod }}}$. A contrast between Co and Au areas is observed caused by $P_{\text {sample, } A u}=0$ and $P_{\text {sample }, C o} \neq 0$. (d) Corresponding profiles along the dashed arrows shown in (c). The data is normalized to $I_{0}$ and an offset is subtracted for clarity: $\mathrm{Au} / \mathrm{Au}$ crossover shows no difference in signal. At the $\mathrm{Au} / \mathrm{Co}$ crossover a difference in signal of $\Delta(3.2 \pm 0.7) \cdot 10^{-4}$ is observed. Artifacts at step edges result from a response of the STM feedback loop in the CCM. 


\section{SP-STM}

with respect to the underlying $\mathrm{Au}(111)$. During this sp-STM investigation at the solid/liquid interface the coil around the AC20 tip end is modulated at a frequency $f_{\text {mod }}=59.7 \mathrm{kHz}$, which is significantly above the cut-off frequency of the STM feedback loop $(20 \mathrm{kHz})$. The topographical profiles plotted in Figure 4.21(b) demonstrate clearly that STM investigations are not influenced by the modulated magnetic field caused by the coil of the sp-STM setup.

The magnetic information is measured simultaneously via lock-in technique at $f_{\text {mod }}=59.7 \mathrm{~Hz}$ using a time constant of $3 \mathrm{~ms}$. The resulting magnetization map of the investigated sample is displayed in Figure 4.21(c) representing the magnitude of the ratio $\frac{\Delta I_{s p}^{f_{\text {mod }}}}{I_{0}}$ at $f_{\text {mod }}$. According to Equation 4.6 this ratio is proportional to the combined spin-polarization $P_{\text {tip }} P_{\text {sample }}$.

In this lock-in image a sharp contrast between extended Co islands and the remaining $\mathrm{Au}(111)$ terraces is observed, whereby the blue highlighted Co area on Au within the topographical STM image is congruent with the darker area of the lock-in image. Both indicated magnetic profiles in Figure 4.21(c) correspond to the ones in the topographical image, and they are plotted in Figure 4.21(d). There, the lock-in data $\Delta I_{s p}^{f_{\text {mod }}}$ is normalized to the setpoint of the tunneling current in constant-current mode $\bar{I}=I_{0}=6 \mathrm{nA}$, and in addition an offset is subtracted for clarity.

No change in signal is observed when single atomic $\mathrm{Au} / \mathrm{Au}$ step edges are traversed: $\frac{\Delta I_{s p o d}^{f_{\text {mod }}}}{I_{0}}(\mathrm{Au} / \mathrm{Au})=\Delta(0.0 \pm 0.4) \cdot 10^{-4}$. Only at the step edges an increase in signal magnitude is observed (marked by gray arrows) caused by the response of the STM feedback loop. Within the exemplary profile across the $\mathrm{Au} / \mathrm{Co}$ step edge a significant difference $\frac{\Delta I_{s p}^{f_{\text {mod }}}}{I_{0}}(\mathrm{Au} / \mathrm{Co})=\Delta(3.2 \pm 0.7) \cdot 10^{-4}$ in signal between $\mathrm{Au}$ and Co area is observed, which leads to the contrast in the lock-in image.

This contrast results from a difference in magnitude of the measured modulated component $I_{s p}^{f_{\text {mod }}}$ of the tunneling current on top of the ferromagnetic Co and the diamagnetic Au surface, respectively. One does not expect a spin-polarized tunneling current at the bare $\mathrm{Au}(111)$ surface, since the spin-polarization of the $\mathrm{Au}(111)$ surface is $P_{\text {sample } A u}=0$. However, the magnetization $\vec{M}_{\|}$parallel to the surface normal $\vec{a}$ at the Co area $\left(P_{\text {sample }, C o} \neq 0\right)$ leads to a measurable contrast. Therefore, this observed $\mathrm{Co} / \mathrm{Au}$ contrast depends on a differing ferromagnetic/diamagnetic surface, not on topography, except for some artifacts at step edges which do not result from spin-polarization. These are related to the response of the STM feedback loop in the constant-current mode.

In a further sp-STM measurement a larger sample area is investigated in detail, as displayed in Figure 4.22(a), starting again with the topographical sp-STM image. Deposited Co is highlighted in blue as a guide to the eyes. The indicated profiles in Figure 4.22(a) along the white dashed lines are shown in Figure 4.22(b 


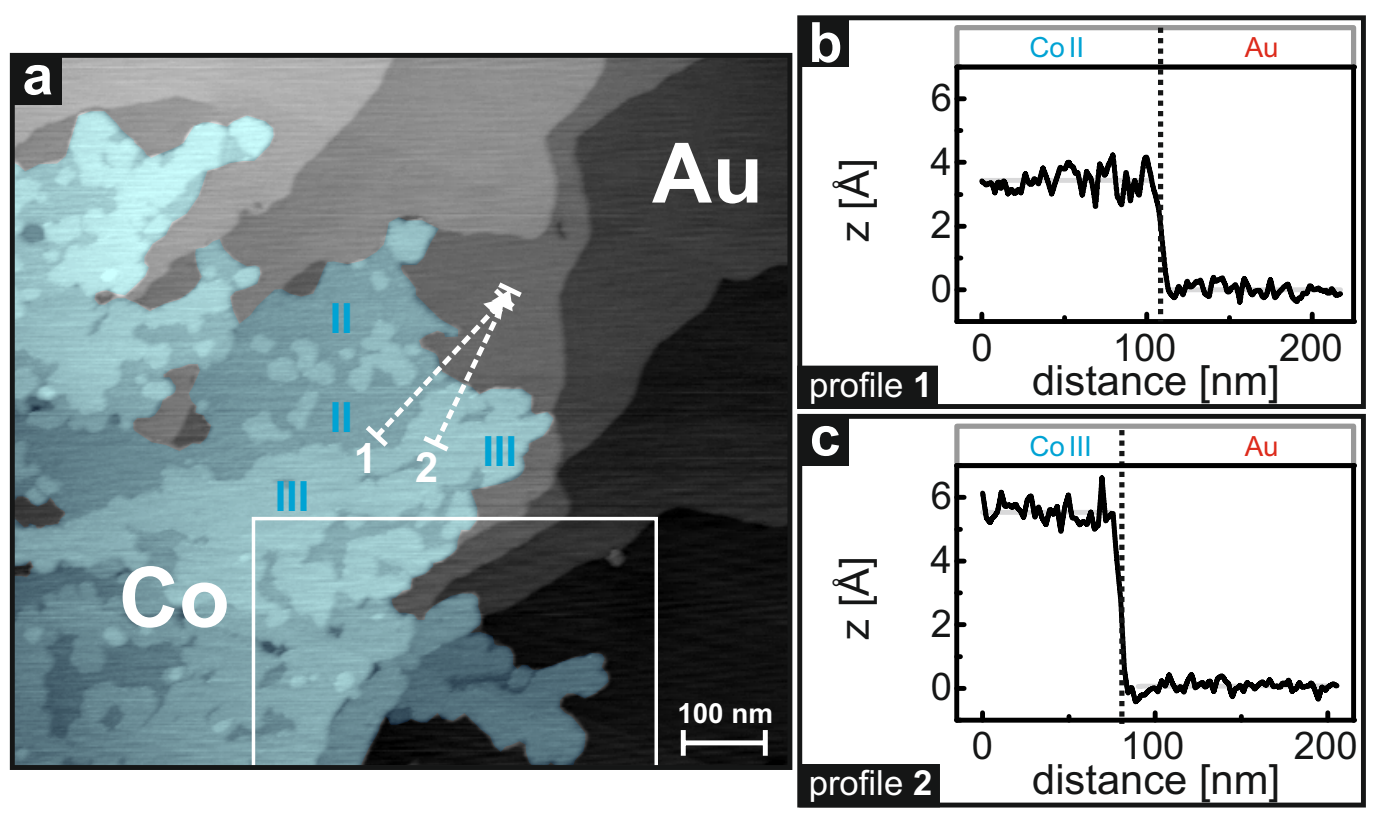

Figure 4.22: Large scale sp-STM of $\mathrm{Co} / \mathrm{Au}(111)$ with an AC20 tip: $I_{0}=6 \mathrm{nA} ; E_{\mathrm{WE}}=$ $-351 \mathrm{mV} ; E_{\text {tip }, \mathrm{AC} 20}=-369 \mathrm{mV} ; f_{\mathrm{mod}}=59.7 \mathrm{kHz}$. (a) Topographical STM image of deposited Co (highlighted in blue, up to $4 \mathrm{AL}$, partially indicated by Roman numerals) on $\mathrm{Au}(111)$. (b \& c) Topographic profiles along the arrows shown in (a) across $\mathrm{Co} / \mathrm{Au}$ step edges of $2 \mathrm{AL} \widehat{=}(3.4 \pm 0.4) \AA$ and $3 \mathrm{AL} \widehat{=}(5.5 \pm 0.3) \AA$ with respect to the $\mathrm{Au}(111)$ terrace underneath. These observations demonstrate that the modulation required for sp-STM does not have an impact on topographical information.

\& c). They correspond to $\mathrm{Co} / \mathrm{Au}$ step edges of $2 \mathrm{AL} \widehat{=}(3.4 \pm 0.4) \AA$ (profile 1$)$ and $3 \mathrm{AL} \widehat{=}(5.5 \pm 0.3) \AA$ (profile 2$)$ with respect to the underlying $\mathrm{Au}(111)$ terrace, respectively.

The corresponding magnetic image to the large scale topographical STM image of deposited $\mathrm{Co}$ on $\mathrm{Au}(111)$, Figure 4.22, is shown in Figure 4.23(a) displaying the simultaneously measured spin-polarized component of the tunneling current $\Delta I_{\text {sp }}^{f_{\text {mod }}}$ at $f_{\bmod }=59.7 \mathrm{kHz}$. Here a major contrast between Co and Au areas is also obvious, as observed in the close-up sp-STM measurement above caused by the different spinpolarization $P_{\text {sample } C \text { Co }} \neq P_{\text {sample }, A u}=0$. Additionally, a slightly different signal is measured within the extended Co island between areas of $2 \mathrm{AL}$ and $3 \mathrm{AL}$ in height. To illustrate this observation more clearly two exemplary profiles across $\mathrm{Co} / \mathrm{Au}$ step edges of $2 \mathrm{AL}$ (profile 1) and $3 \mathrm{AL}$ (profile 2) with respect to the $\mathrm{Au}(111)$ terrace are selected in the lock-in image, see Figure $4.23(\mathrm{~b} \& \mathrm{c})$. In these profiles a signal difference $\Delta(3.1 \pm 0.7) \cdot 10^{-4}$ is measured for $\mathrm{Co} / \mathrm{Au} 2 \mathrm{AL}$ and $\Delta(3.3 \pm 0.7) \cdot 10^{-4}$ for $\mathrm{Co} / \mathrm{Au} 3 \mathrm{AL}$.

The uncertainties of the measured values are minimized upon averaging mul- 

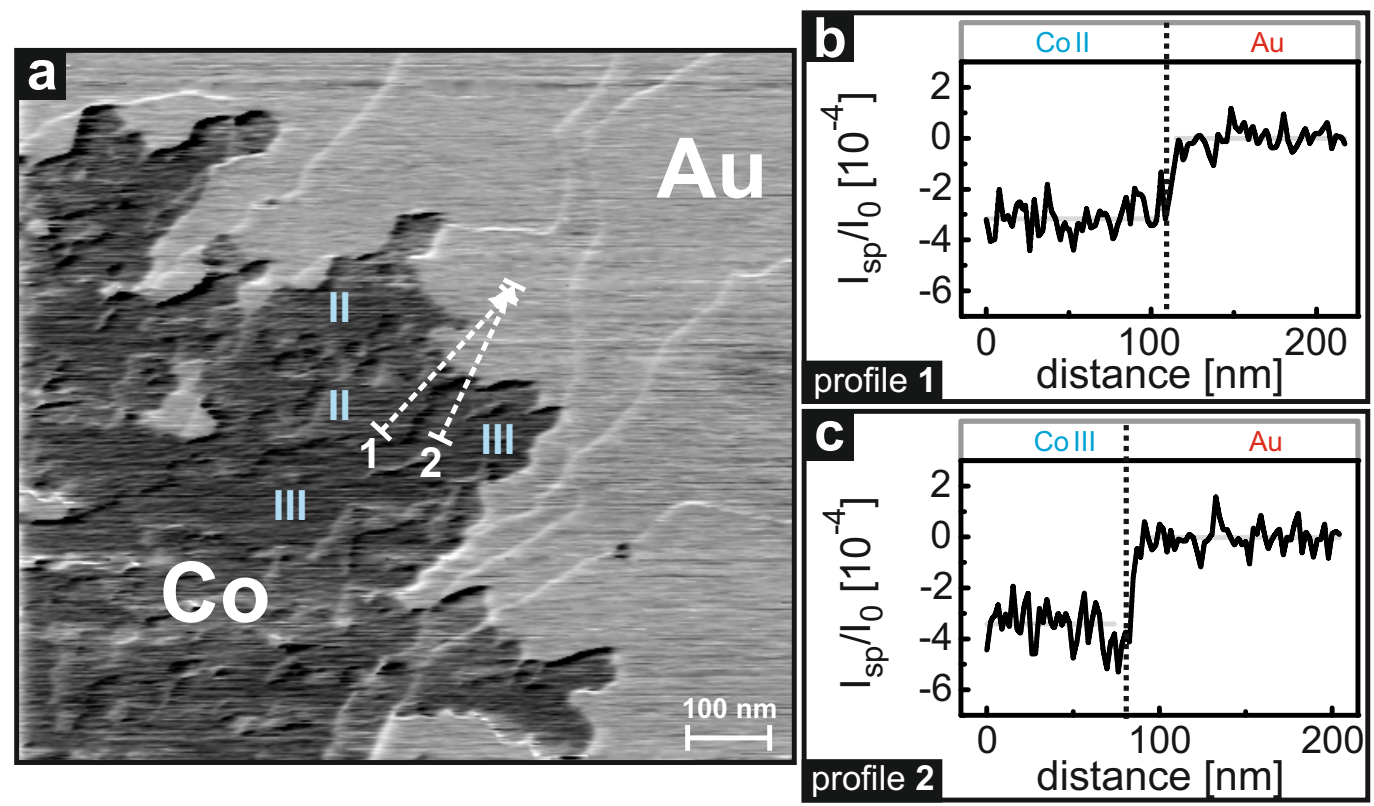

Figure 4.23: Large scale sp-STM of $\mathrm{Co} / \mathrm{Au}(111)$, corresponding $\Delta I_{s p}^{f_{\text {mod }}}: I_{0}=6 \mathrm{nA}$; $E_{\mathrm{WE}}=-351 \mathrm{mV} ; E_{\mathrm{tip}, \mathrm{AC} 20}=-369 \mathrm{mV} ; f_{\mathrm{mod}}=59.7 \mathrm{kHz}$. (a) Simultaneously measured spin-polarized component of the tunneling current at $f_{\text {mod }}$. A clear contrast between $\mathrm{Co}$ and $\mathrm{Au}$ areas is observed, caused by the spin-polarized tunneling current $I_{s p}^{f_{\text {mod }}}$. Additionally, a low contrast between $2 \mathrm{AL}$ and $3 \mathrm{AL}$ Co is observed within the extended Co island, exemplarily indicated by Roman numerals. Artifacts at step edges result from a response of the STM feedback loop in the constant-current mode. (b \& c) Corresponding magnetic profiles across $\mathrm{Co} / \mathrm{Au}$ step edges indicated in (a). The data is normalized to $I_{0}$ and an offset is subtracted for clarity: for Co/Au $2 \mathrm{AL}$ here the difference in value is $\Delta(3.1 \pm 0.7) \cdot 10^{-4}$ and for $\mathrm{Co} / \mathrm{Au} 3 \mathrm{AL}$ it is $\Delta(3.3 \pm 0.7) \cdot 10^{-4}$, as displayed by the linear fits (gray lines).

tiple profiles across such $\mathrm{Co} / \mathrm{Au}$ step edges. Thus, a difference in ratio $\frac{\Delta I_{s p}^{f_{\text {mod }}}}{I_{0}}$ is observed of $\Delta(2.92 \pm 0.30) \cdot 10^{-4}$ for $2 \mathrm{AL}$ step edges and $\Delta(3.15 \pm 0.31) \cdot 10^{-4}$ for $3 \mathrm{AL} \mathrm{Co} / \mathrm{Au}$ step edges. These differences can be observed by a low contrast within the Co area, Figure 4.23(a), even though it is close to the current resolution of the measurement, given by the lock-in sensitivity. This observation indicates, that the alignment of magnetization $\vec{M}$ with respect to the surface normal $\vec{a}$ depends on the actual Co layer thickness.

To verify the sp-STM measurements on $\mathrm{Co} / \mathrm{Au}(111)$, control experiments are done using pure and non-magnetic Au tips instead of magnetic AC20 tips, but under similar sp-STM setup conditions. Thus, the coil around the Au tip end generates a modulated magnetic field as described above. Since the Au tip is diamagnetic its spin-polarization is $P_{t i p, A u}=0$, therefore according to Equation 4.6 

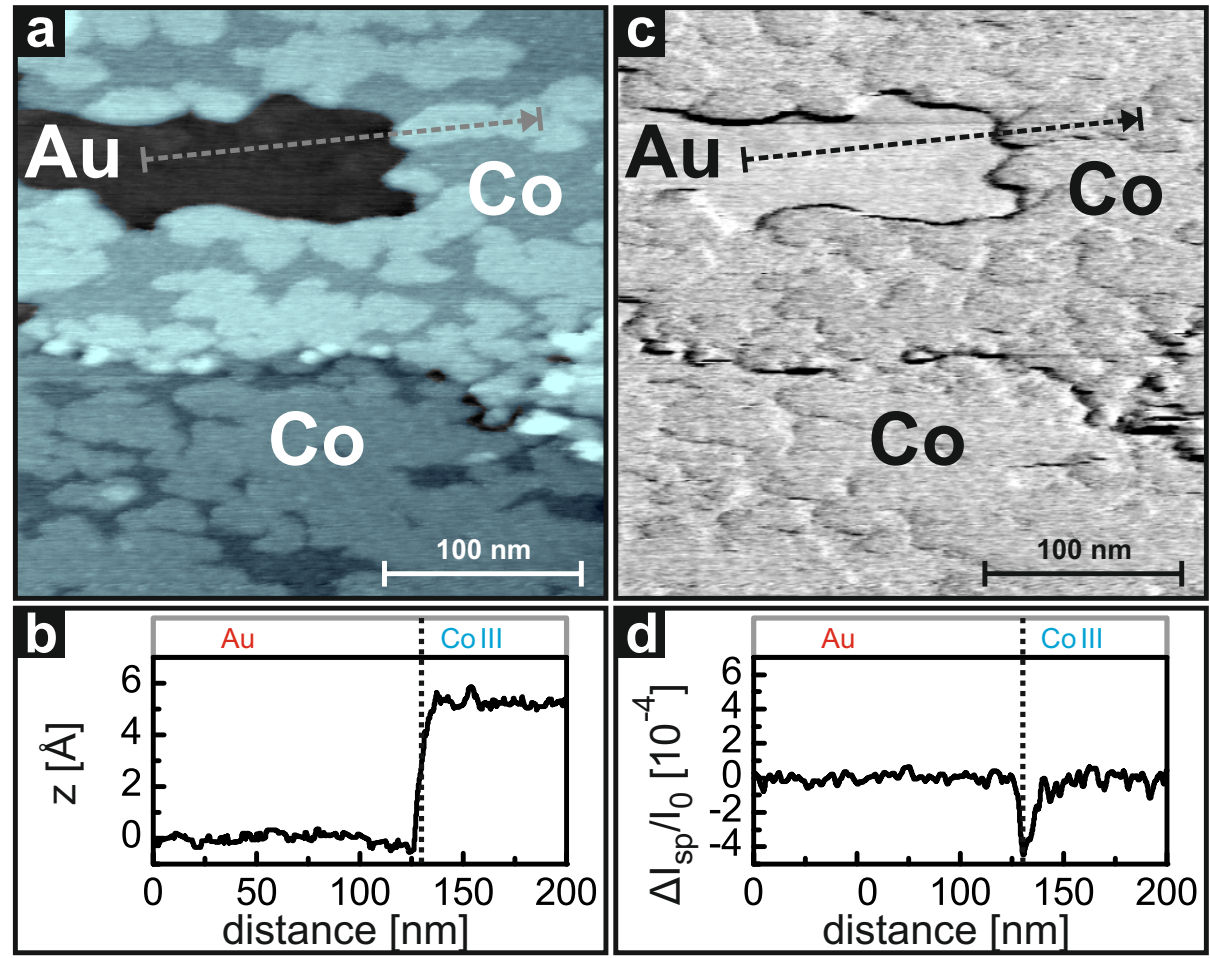

Figure 4.24: Sp-STM control experiment at $\mathrm{Co} / \mathrm{Au}(111)$ using an $\mathrm{Au}$ tip. $I_{0}=5 \mathrm{nA}$; $E_{\mathrm{WE}}=-349 \mathrm{~V} ; E_{\mathrm{tip}, \mathrm{Au}}=-359 \mathrm{~V} ; f_{\mathrm{mod}}=37.7 \mathrm{kHz}$. (a) Topographical STM image of up to $4 \mathrm{AL}$ of electrodeposited $\mathrm{Co}$ (blue) and pristine $\mathrm{Au}(111)$ areas. (b) $\mathrm{An}$ exemplary profile across an $\mathrm{Au} / \mathrm{Co}$ step edge of $3 \mathrm{AL} \widehat{=}(5.3 \pm 0.3) \AA$ is plotted for comparison with investigations above. (c) Simultaneously measured lock-in signal of the tunneling current at $f_{\text {mod }}$. No difference in signal between Co and Au areas is observed because of $P_{t i p, A u}=0$. Only step edges are displayed as caused by a response of the STM feedback loop in the constant-current mode. (d) The corresponding magnetic profile underlines that there is no difference in the lock-in signal between Co and $\mathrm{Au}$ areas: $\Delta \frac{\Delta I_{s p}^{f_{\text {mod }}}}{I_{0}}(\mathrm{Au} / \mathrm{Co})=(0.0 \pm 0.4) \cdot 10^{-4}$.

no spin-polarized component of the tunneling current is expected. Such a sp-STM control experiment is shown in Figure 4.24. A sample surface partially covered by electrodeposited Co and remaining $\mathrm{Au}$ areas is prepared similar to sp-STM investigations above. The topographical STM image is displayed in Figure 4.24(a), where a topographical profile across a $3 \mathrm{AL} \mathrm{Co} / \mathrm{Au}$ step edge is taken in order to facilitate comparison to the above mentioned investigations. This exemplary profile is plotted in Figure 4.24(b) and shows an identical step height of $(5.3 \pm 0.2) \AA$ as in Figure 4.21(b). The corresponding lock-in image of the control experiment is shown in Figure 4.24(c). No difference in the lock-in signal between $\mathrm{Co}$ and $\mathrm{Au}$ areas is observed when a non-ferromagnetic Au tip is used. The corresponding profile across 


\section{SP-STM}

the indicated $3 \mathrm{AL} \mathrm{Au/Co} \mathrm{step} \mathrm{edge,} \mathrm{plotted} \mathrm{in} \mathrm{Figure} \mathrm{4.24(d),} \mathrm{underlines} \mathrm{this} \mathrm{ob-}$ servation. The artifacts induced by step edges still outline the surface morphology, even though no contrast between $\mathrm{Co}$ and $\mathrm{Au}$ is evident.

These sp-STM measurements on $\mathrm{Co} / \mathrm{Au}(111)$ utilizing AC20 tips demonstrate that the investigated extended Co islands feature a magnetization component in out-of-plane direction, thus magnetization parallel to the surface normal is observed $\left(\vec{M}_{\|} \neq 0\right)$.

No domain borders could be observed within extended Co islands. This is not surprising, since the size of the extended Co islands is around $0.25 \mu^{2}$, which is much smaller than the typical lateral size of single domains $\left(5 \mu \mathrm{m}^{2}\right)$ in perpendicular magnetized Co of similar thickness [1].

Slightly different $\frac{\Delta I_{s p}^{f_{\text {mod }}}}{I_{0}}$ values in dependence on the actual Co layer thickness give a hint to differently aligned magnetization $\vec{M}$ with respect to the surface normal $\vec{a}$.

The influence of the solid/liquid interface on the spin-polarization of the tunneling current seems to be a remarkable, fundamental aspect in the experiments. Previous studies [92, 93] of the tunneling gap at the solid/liquid interface have shown that the tunneling process at high tunneling current setpoints occurs across a vacuum-like gap, as detailed in Section 3.2 93. The gap width at high tunneling currents is too small as to allow for $\mathrm{H}_{2} \mathrm{O}$ molecules - which are of a size smaller than any other molecule or solvated ion in the electrolyte - to penetrate into the tunneling gap.

\subsection{Effects at the Second Harmonic}

Measurements of the modulated component of the tunneling current at the modulation frequency's second harmonic are used to investigate the susceptibility of a magnetic substrate [47, 48, 204]. Thereby, not only magnetization of the magnetic tip is manipulated by the coils magnetic field, but also magnetization of the investigated sample surface is affected by stray magnetic fields of the tip and/or the coil. In addition to the sp-STM observations of $\mathrm{Co} / \mathrm{Au}(111)$ presented in Section 4.4. in the following the modulated component of the tunneling current at $\left(2 f_{\text {mod }}\right)$ is investigated. Two sources of origin for a modulated component of the tunneling current at $\left(2 f_{\mathrm{mod}}\right)$ are possible. On the one hand the magnetostriction effect of the magnetic AC20 tip occurs at $\left(2 f_{\text {mod }}\right)$ which is the topic of the first section. On the other hand an influence of an external magnetic field on the magnetization of the sample under investigation is subject of further discussion. 


\subsubsection{Magnetostriction}

The modulated magnetostriction of a magnetic tip $\Delta L$ causes a modulated tipsubstrate distance $d$, as already detailed in Section 4.3. This change is not observed in sp-STM measurements in CCM $\bar{I}$, however, signals in the tunneling current $I$ at higher frequencies are observed by lock-in technique. Since the modulation frequency $f_{\text {mod }}$ is adjusted to around $40 \mathrm{kHz}$ a signal by magnetostriction is present in the tunneling current at $1 f_{\text {mod }}$ and $2 f_{\text {mod }}$. Therefore, the tunneling current $I$ can be expressed by a time averaged component $\bar{I}=I_{0}$, a spin-polarized component

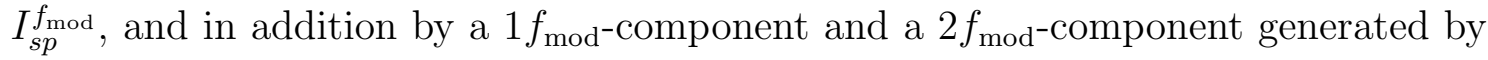
magnetostriction

$$
I=I_{0}+I_{s p}^{f_{\text {mod }}}+I_{\lambda, \text { part }}^{f_{\text {mod }}}+I_{\lambda, \text { sat }}^{2 f_{\text {mod }}}
$$

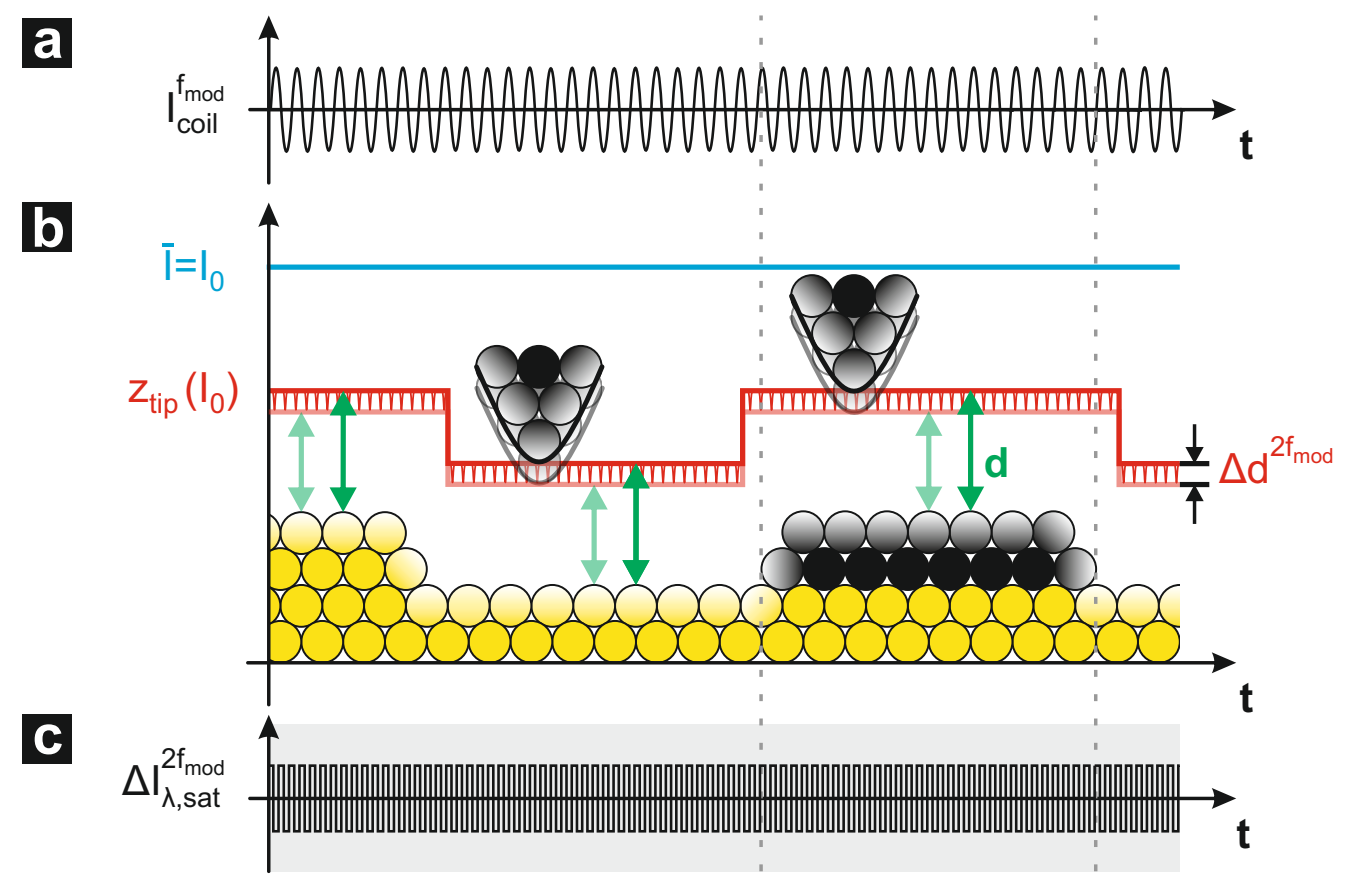

Figure 4.25: During sp-STM measurements the saturated magnetostriction effect of an AC20 tip generates a tunneling current component at $\left(2 f_{\text {mod }}\right)$ caused by the modulated change of the tip-substrate distance $\Delta d^{2 f_{\text {mod }}}$. An offset $\left|I_{\lambda \text {, sat }}^{2 f_{\text {mod }}}\right|$ is observed, since this signal results from the magnetic tip throughout the duration of the modulation independently of the sample.

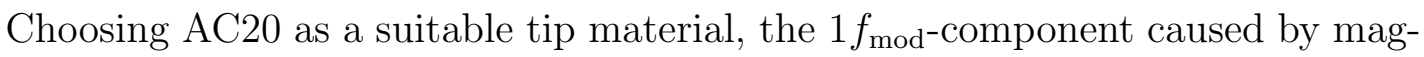
netostriction vanishes, since its magnetostriction occurs at $2 f_{\text {mod }}$ when the magnetic field strength is set to $\left|H_{z}\right|>30$ Oe according to magnetostriction investigations, 


\section{SP-STM}

see Section 4.3.3.

$$
I=I_{0}+I_{s p}^{f_{\text {mod }}}+I_{\lambda, \text { sat }}^{2 f_{\text {mod }}} \text {, for AC20 and }\left|H_{z}\right|>30 \text { Oe }
$$

An illustration of sp-STM measurements via lock-in technique at $\left(2 f_{\text {mod }}\right)$ is illustrated in Figure 4.25. Caused by a modulated magnetic field at $f_{\text {mod }}$, a magnetostrictively modulated difference in tip-surface distance $\Delta d$ occurs at $2 f_{\text {mod }}$, which is measured by lock-in technique. The magnetostrictive effect of the AC20 tip takes place throughout the entire duration of the sp-STM measurement, but regardless of the actual sample surface under investigation.

This effect of magnetostriction is independent of a spin-polarized signal between tip and surface, since it originates from the magnetic tip. Therefore, the same signal is expected whether the sample surface is magnetized in out-of plane direction or not.

However, magnetostriction causes an influence on the averaged tunneling current and thus on the tip-surface distance $d$ adjusted by the STM feedback loop in CCM. Because of this, signals caused by magnetostriction should be as low as possible to enable sp-STM measurements.

\subsubsection{Magnetic Susceptibility}

The magnetic susceptibility $\chi$ describes the magnetization of a sample depending on an external field and is in general given by a tensor

$$
\chi_{i j}=\frac{\delta M_{i}}{\delta H_{j}}, \text { with } i=x, y, z \text { and } j=x, y, z .
$$

Regarding the setup $\boldsymbol{b}$ used to investigate tip magnetostriction, where the coil is positioned around tip apex and sample, the magnetization of magnetic sample would be affected. But also when sp-STM setup $\boldsymbol{a}$ is used, where the coil is installed at the tip holder around the upper part of the tip, an influence of stray magnetic fields from the tip and/or the coil itself on the magnetic sample surface can not be excluded, even if the magnitude of the magnetic field is set as low as possible.

Such an effect on a magnetic sample is illustrated in Figure 4.26. Figure 4.26(a) shows a model of sp-STM of an out-of plane magnetized sample, where the magnetization is constantly tilted by an angle $\alpha$ by switching only the tip magnetization, as discussed in Section 4.1. An externally applied magnetic field leads to a deflection of the magnetization away from $\alpha$. In case of sp-STM, where the magnetic field of the coil is modulated at $f_{\text {mod }}$, the out-of-plane component of sample magnetization is manipulated depending on time as well, see Figure 4.26(b).

According to Equation(4.3), the tunneling current $I$ is given by

$$
I=I_{0}+I_{0} P_{A} P_{B} \cos \left[\theta+\alpha\left(f_{\text {mod }}\right)\right]=I_{0}+I_{s p},
$$


a

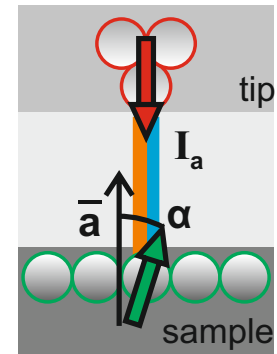

$\theta=180^{\circ}+\alpha$

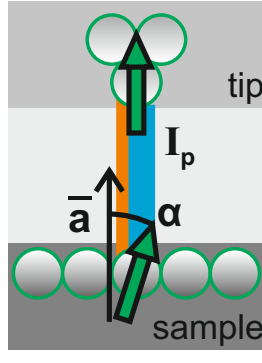

$\theta=0^{\circ}+\alpha$
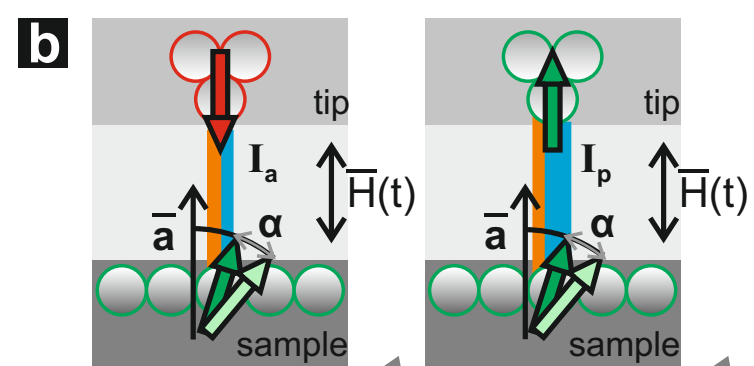

$\theta=0^{\circ}+\alpha(t)$

Figure 4.26: Illustration of anti-/parallel tip-sample orientation, where the sample magnetization is tilted by angle $\alpha$ with respect to the surface normal $\vec{a}$ (a) constant in time. (b) in dependence on an external field $\vec{H}$, which is modulated in time corresponding to $f_{\text {mod }}$.

neglecting components of the tunneling current generated by tip magnetostriction, which would cause an offset. Consequently, a spin-polarized component of the tunneling current can be divided into anti-/parallel orientation parallel and antiparallel orientation $I_{p}$ and $I_{a}$, respectively:

$$
I_{s p}= \begin{cases}I_{a}(t)=I_{0} \cdot\left(1-P_{A} P_{B} \cos \left[\alpha\left(f_{\text {mod }}\right)\right]\right), & \theta=180^{\circ} \\ I_{p}(t)=I_{0} \cdot\left(1+P_{A} P_{B} \cos \left[\alpha\left(f_{\text {mod }}\right)\right]\right), & \theta=0^{\circ}\end{cases}
$$

However, in either case the additionally modulated sample magnetization causes a modulated anti-/parallel orientation. The origin of spin-polarized components of the tunneling current at $f_{\text {mod }}$ as well as $\left(2 f_{\text {mod }}\right)$ is illustrated in Figure 4.27. In this model it is assumed that the tip is a soft ferromagnet (narrow hysteresis) and the magnetic sample under investigation is a hard ferromagnet (wide hysteresis). Thereby, the tip magnetization $\vec{M}_{t i p}$ is switched by the magnetic field between $\theta=0^{\circ}$ and $180^{\circ}$ with respect to the surface normal $\vec{a}$, when sample magnetization $\vec{M}_{\text {sample }}$ is deflected from angle $\alpha$ with respect to the surface normal $\vec{a}$. This provides a realistic estimate, according to SQUID investigations on AC20 (soft ferromagnetic), see Figure 4.14. and compared to magnetization curves of $\mathrm{Co} / \mathrm{Au}(111)$ (hard ferromagnetic) [34, 73].

Therefore, in Figure 4.27 (b) the tip magnetization $\vec{M}_{t i p}$ parallel to the surface normal $\vec{a}$ is idealized by a square wave and the sample magnetization $\vec{M}_{\text {sample }}$ by a shifted sinusoidal modulation, both correlated to a modulation frequency $f_{\text {mod }}$ of the external magnetic field $\vec{H}$, see Figure 4.27(a). The term $\cos (\theta+\alpha)$ of the total angle $\theta+\alpha$ is a projection of both magnetizations $\vec{M}_{t i p}$ and $\vec{M}_{\text {sample }}$ on the surface normal $\vec{a}$, and considering Equation (4.19) this term is proportional to the product of the combined spin-polarization $P_{\text {tip }} P_{\text {sample }}$, exemplary illustrated in Figure 4.27(c). This 

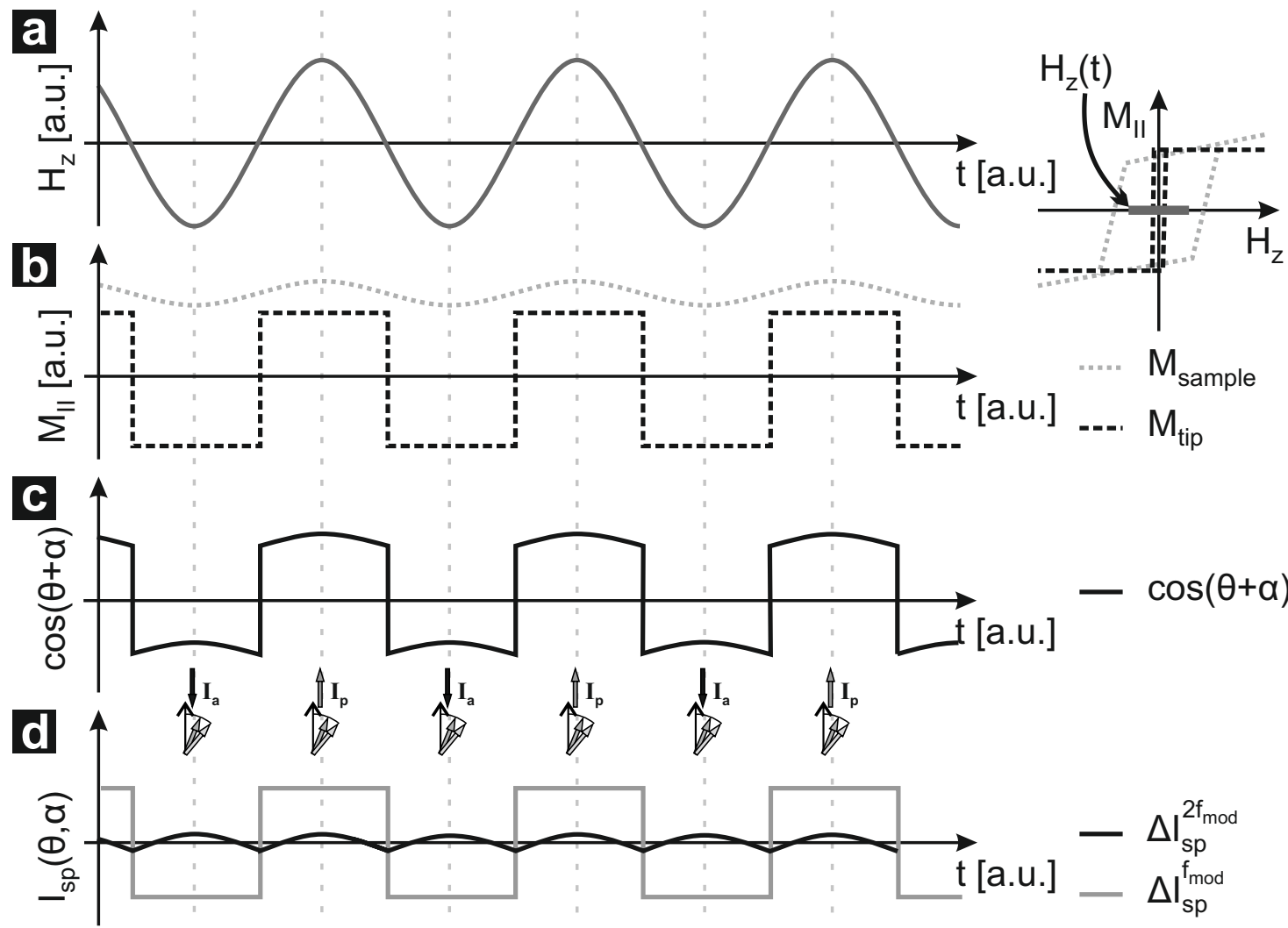

Figure 4.27: Illustrated origin of $\Delta I_{s p}^{2 f_{\text {mod }}}$ using a soft ferromagnetic tip and a hard ferromagnetic sample: (a) Modulation of an external field with $H_{z}\left(f_{\bmod }\right)$. (b) Switching of tip magnetization $\vec{M}_{t i p}$ is approximated by a square wave. Here, sample magnetization $\vec{M}_{\text {sample }}$ is affected in addition, approximated by a sinusoidal modulation, which is related to angle $\alpha\left(f_{\text {mod }}\right)$ with respect to the surface normal $\vec{a}$. (c) Corresponding projection on the $z$-axis by $\cos (\theta+\alpha)$, proportional to $P_{\text {tip }} P_{\text {sample }}$. (d) $\cos (\theta+\alpha)$ can be described by a square wave at $f_{\text {mod }}$ in addition with modulation showing a strong component at $\left(2 f_{\text {mod }}\right)$.

resulting modulated square wave can be expressed by a superposition of a square wave with $f_{\text {mod }}$, representing the spin-polarized component of the tunneling current at $f_{\text {mod }}$, discussed in Section 4.2.1, and an additional modulation showing a strong component at $\left(2 f_{\text {mod }}\right)$. Both originate from spin-polarization and are illustrated in Figure 4.27(d).

A model similar to this one is discussed by Ding [49], where it is assumed that the sample magnetization changes between two states instead of being linearly dependent on an external field $\vec{H}$. Therefore, a modulated sample magnetization $\vec{M}_{\text {sample }}$ is approached by a shifted square wave of low magnitude. A spin-polarized signal component at $\left(2 f_{\text {mod }}\right)$ occurs as well, since the modulated magnetizations of tip and 
sample are phase shifted to each other caused by the differing magnetic properties both.

\subsubsection{Experiment}

In order to prevent magnetization oscillations of the substrate, the externally applied magnetic field to switch the tip magnetization should be as small as possible. Therefore, sp-STM investigations detecting a $f_{\text {mod }}$-signal component of tunneling current are made by sp-STM setup $\boldsymbol{a}$ introduced in Section 4.2 .

The identical setup can be utilized to investigate a component of the tunneling current at the second harmonic, but now the signal is detected by lock-in technique at $\left(2 f_{\text {mod }}\right)$, as illustrated in Figure 4.28 . In this scenario it is assumed, that the stray

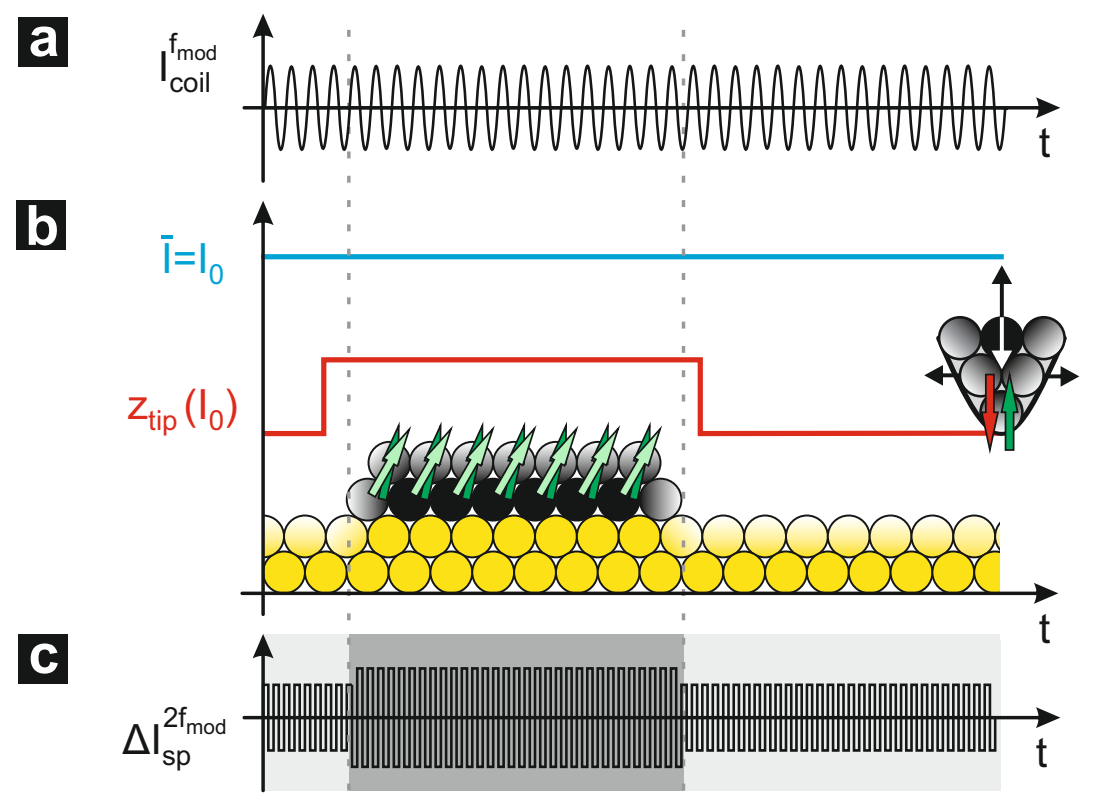

Figure 4.28: Illustrated sp-STM at $\left(2 f_{\text {mod }}\right)$ : (a) Tip magnetization $\vec{M}_{\text {tip }}^{f_{\text {mod }}}$ is switched periodically by the magnetic field of a current modulated coil at $f_{\text {mod }}$. (b) The time averaged tunneling current is equal to the set-point in CCM: $\bar{I}=I_{0}$. (c) Since both the tip magnetization and the sample magnetization are affected, the alternating magnetoresistance causes a modulated spin-polarized component of the tunneling current at $\left(2 f_{\text {mod }}\right)$. The detection of $\Delta I_{s p}^{2 f_{\text {mod }}}$ is accomplished via lock-in technique.

magnetic fields of both tip and/or coil affecting the investigated magnetic sample as well. In contrast, for sp-STM in differential magnetic mode it is assumed that only a ferromagnetic tip is affected by the modulated magnetic field of the coil.

Corresponding to the sp-STM measurement at $f_{\text {mod }}$ presented in Figure 4.22 and 4.23 , a sp-STM measurement of the same sample area detected at $\left(2 f_{\text {mod }}\right)$ is 


\section{SP-STM}

presented in Figure 4.29 (topography) and Figure 4.30 (corresponding lock-in signal). The observed topography of $\mathrm{Co} / \mathrm{Au}(111)$ is similar to previous investigations shown

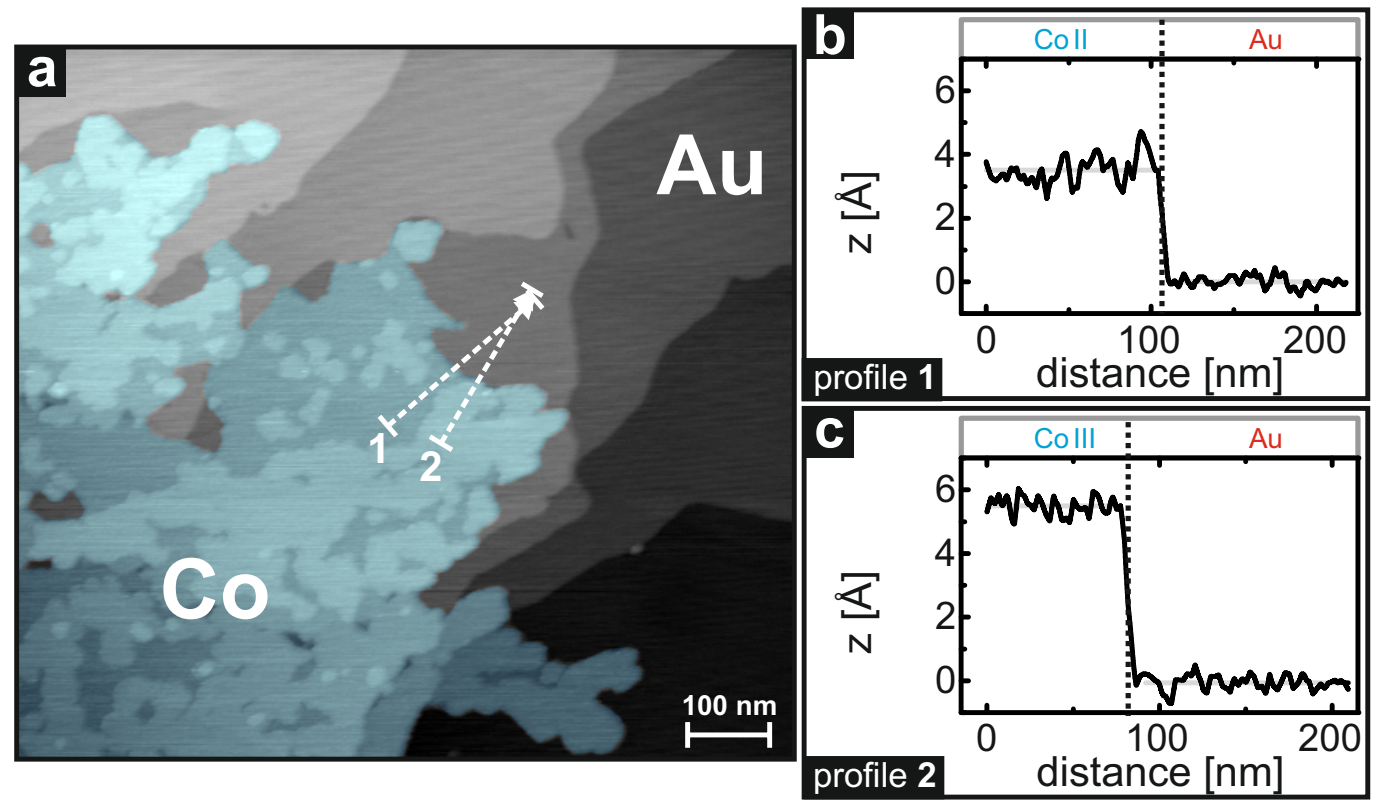

Figure 4.29: Sp-STM at $\left(2 f_{\mathrm{mod}}\right): I_{0}=6 \mathrm{nA} ; E_{\mathrm{WE}}=-351 \mathrm{mV} ; E_{\mathrm{tip}, \mathrm{AC} 20}=$ $-369 \mathrm{mV} ; f_{\text {mod }}=59.7 \mathrm{kHz}$. (a) Topography of deposited Co (blue) on $\mathrm{Au}(111)$. (b \& c) Topographic profiles across Co/Au step edges along the arrows shown in (a) of $2 \mathrm{AL} \widehat{=}(3.5 \pm 0.5) \AA \mathrm{Co}$ and $3 \mathrm{AL} \widehat{=}(5.5 \pm 0.3) \AA$ Co with respect to the $\mathrm{Au}(111)$ terrace. The height of the step edges is similar to previous observations.

in Figure 4.22(a). Two exemplary profiles are selected for comparison, representing Co step edges of $2 \mathrm{AL} \widehat{=}(3.5 \pm 0.5) \AA$ and $3 \mathrm{AL} \widehat{=}(5.5 \pm 0.3) \AA$ with respect to the $\mathrm{Au}(111)$ terrace underneath, plotted in Figure $4.29(\mathrm{~b} \& \mathrm{c})$. The height of these $\mathrm{Co} / \mathrm{Au}$ step edges is similar to previous observations, see Figure 4.22 (b \& c).

Correspondingly, the component of the tunneling current at $\left(2 f_{\text {mod }}\right)$ is mapped in Figure 4.23(a). A difference between extended $\mathrm{Co}$ islands and $\mathrm{Au}(111)$ terraces is observed, even through the signal is much lower. Exemplary profiles at Co step edges with $2 \mathrm{AL}$ and $3 \mathrm{AL}$ height are plotted in Figure $4.30(\mathrm{~b} \& \mathrm{c})$, where the measured values $\Delta I_{s p}^{2 f_{\text {mod }}}$ are normalized to the set-point of the tunneling current $I_{0}$. Additionally, an offset is subtracted for clarity. For both profiles similar differences in signal between $\mathrm{Co} / \mathrm{Au}$ are observed, for $2 \mathrm{AL}(-0.11 \pm 0.07) \cdot 10^{-4}$ and for $3 \mathrm{AL}$ $(-0.12 \pm 0.09) \cdot 10^{-4}$.

While an offset throughout the entire measurements could theoretically originate from magnetostriction, as discussed in Section 4.5.1, a difference signal between Co and $\mathrm{Au}$ can only be caused by spin-polarization. The influence of the stray magnetic fields on the sample magnetization is negligible, since the signal of the tunneling cur- 


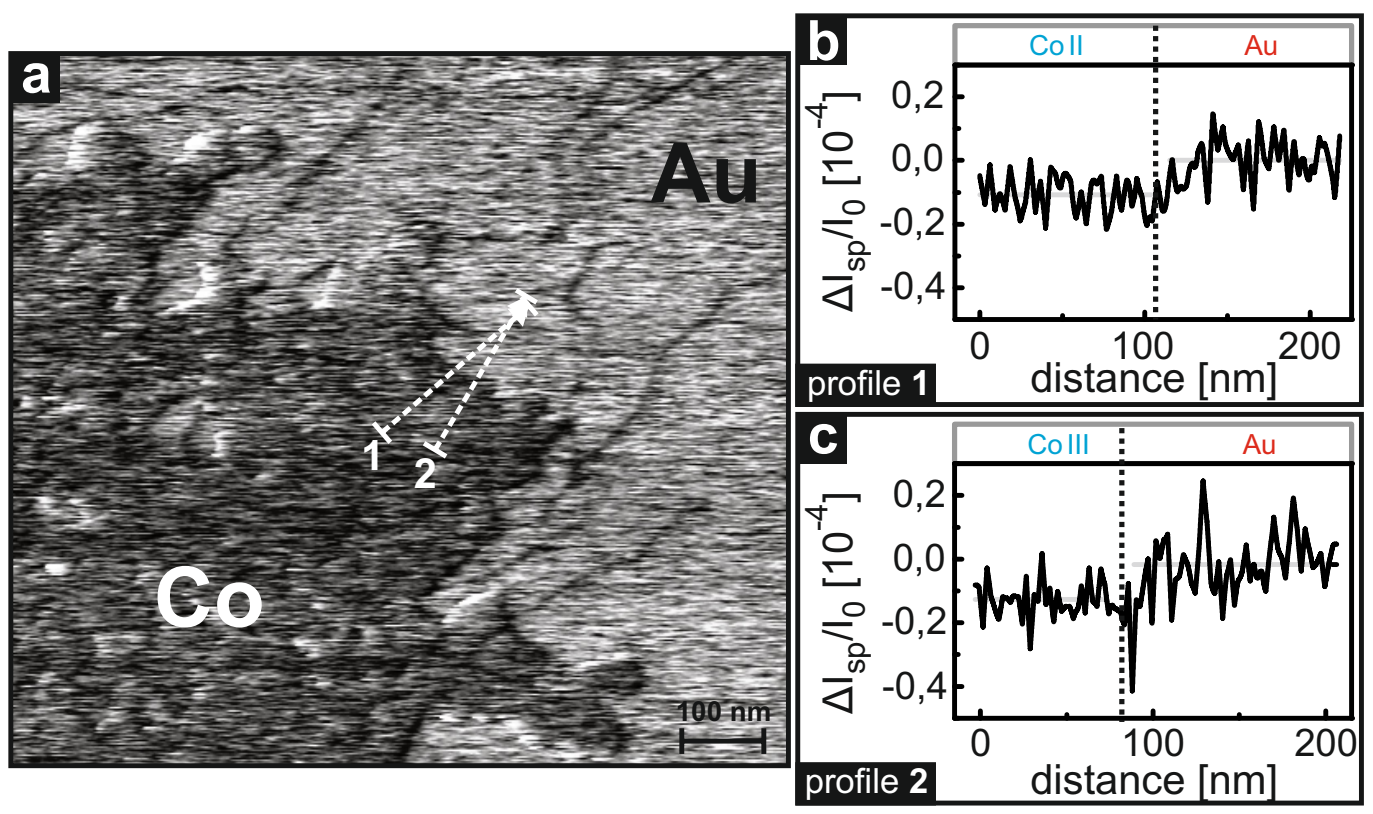

Figure 4.30: Sp-STM at $\left(2 f_{\mathrm{mod}}\right): I_{0}=6 \mathrm{nA} ; E_{\mathrm{WE}}=-351 \mathrm{mV} ; E_{\mathrm{tip}, \mathrm{AC} 20}=$ $-369 \mathrm{mV} ; f_{\text {mod }}=59.7 \mathrm{kHz}$. (a) Simultaneously measured modulated component of the tunneling current at $2 f_{\bmod }=120 \mathrm{kHz}$. The low contrast between Co and $\mathrm{Au}$ at $2 f_{\text {mod }}$ is related to susceptibility. Absence of domain walls within the extended Co island confirms single domain. (b \& c) Corresponding magnetic profiles along the arrows shown in (d). The data is normalized to the setpoint of the tunneling current in constant current mode $I_{0}=6 \mathrm{nA}$ and an offset is subtracted for clarity. No difference is observed between $2 \mathrm{AL}$ and $3 \mathrm{AL}$.

rent component $\Delta I_{s p}^{2 f_{\text {mod }}}$ is $\sim 25 \times$ smaller compared to the spin-polarized tunneling current component $\Delta I_{s p}^{f_{\text {mod }}}$ observed at $f_{\text {mod }}$.

Movements of magnetic domain walls within the deposited Co islands of $2 \mathrm{AL}$ and $3 \mathrm{AL}$ on $\mathrm{Au}(111)$ are not observed, as described elsewhere [49, 201]. This underlines the assumption, that the investigated, extended $\mathrm{Co}$ islands on $\mathrm{Au}(111)$ consist of single domains.

\subsection{Conclusion}

The present electrochemical STM setup was modified to implement sp-STM in differential magnetic mode. Therefore, a magnetically shielded coil, electrically grounded, was constructed and permanently installed at the STM scanner unit positioned around the tip end. An electrical contact between the aluminum coil body and the sp-STM tip in use was avoided by an additionally inserted MACOR tube. This sp-STM is sensitive to magnetized samples in out-of-plane direction due to setup 


\section{SP-STM}

and the tip shape.

For sp-STM a ferromagnetic tip material with small hysteresis loop was desired in order to ensure a switching of the tip magnetization by the small coil around the upper part of the tip. Two different setups were realized to better observe the magnetostriction of different magnetic tip materials by STM. In this way one can make sure, that the magnetostrictive material is affected by the magnetic field. Since the STM setup is highly sensitive to the tip-sample separation $d$, magnetostriction was observed even if the induced length variation is in the angstrom range. Through such STM measurements the magnetostriction of different tip materials was investigated directly. Due to the thin Ni coating all NiAu tips had a significantly smaller magnetostrictive length than $15 \mathrm{~mm}$ long tips of pure Ni.

However, for sp-STM coercivity also plays an important role and especially coated NiAu tips showed a high coercivity, regarding the investigations by SQUID measurements. Therefore the use of pure Ni tips as well as Ni coated Au tips is not recommended for sp-STM, since otherwise high magnetic field strengths are required to switch the tip magnetization during modulation. But the higher the magnetic field strength the more magnetic samples are influenced, and thus the sp-STM measurement will be affected by further effects. AC20 fits all sp-STM requirements low coercivity and vanishing magnetostriction.

As a model system extended Co islands were electrochemically grown on $\mathrm{Au}(111)$, since thin films of Co show an easy-axis of magnetization in out-of plane direction caused by magnetocrystalline anisotropy. These samples showed areas of ferromagnetic Co and diamagnetic $\mathrm{Au}$, which is an ideal surface for investigations by sp-STM at the solid/liquid interface.

A modulated magnetic field, generated by the modulated driven coil with a modulation frequency $f_{\text {mod }} \gg f_{\mathrm{STM}}$, was used to manipulate the tip magnetization periodically in direction of the surface normal $\vec{a}$, the time averaged tunneling current $\bar{I}$ was not affected. Consequently, sp-STM measurements are accomplished using AC20 tips without impact on topography. The corresponding signal of the modulated component of the tunneling current $\Delta I_{s p}^{f_{\text {mod }}}$ displayed a sharp contrast between $\mathrm{Co}$ and $\mathrm{Au}$ areas. This is caused by the different spin-polarizations of the surface $P_{\text {sample }, \text { Co }} \neq P_{\text {sample }, A u}=0$.

Sp-STM control experiments were done using a non-magnetic Au tip instead of a magnetic AC20 tip, thus the tip spin-polarization is $P_{t i p, A C 20} \neq P_{t i p, A u}=0$ and the control measurements showed no contrast between Co and Au.

It was demonstrated, that sp-STM at the solid/liquid interface is accomplished, since the tunneling process occurs across a vacuum-like gap and electron spin is conserved. This way, the magnetization of a sample at the solid/liquid interface can be investigated locally resolved. In addition, hints on a Co spin-reorientation transition are observed depending on the Co layer thickness.

To complement sp-STM investigations on $\mathrm{Co} / \mathrm{Au}(111)$, sp-STM measurements at 
$\left(2 f_{\text {mod }}\right)$ were presented, corresponding to prior $\mathrm{Co} / \mathrm{Au}(111)$ investigations at $f_{\text {mod }}$. There, a much smaller difference in signal above $\mathrm{Co}$ areas and $\mathrm{Au}(111)$ terraces was observed. Two possible sources of origin were discussed. A component of the tunneling current at $\left(2 f_{\text {mod }}\right)$ can originate from magnetostriction. However, this is an effect of the tip, which occurs throughout the entire sp-STM measurement independently of the investigated sample and regardless of sample magnetization. Therefore, it is not expected that a magnetostrictive tip can cause a difference in the lock-in signal between Co and Au areas neither at $\Delta I_{s p}^{f_{\text {mod }}}$ nor $\Delta I_{s p}^{2 f_{\text {mod }}}$. Is much more reasonable that an additional modulation of the sample magnetization affected by magnetic stray fields of the magnetic sp-STM tip or the coil would generate a spin-polarized component of the tunneling current at $\left(2 f_{\bmod }\right)$.

Despite the fact that such a signal was observed, compared to the magnitude of the spin-polarized signal at $\left(1 f_{\mathrm{mod}}\right)$ this one at $\left(2 f_{\mathrm{mod}}\right)$ is $\sim 25$ times smaller. Therefore, an influence of the sp-STM setup $\boldsymbol{a}$ in use on deposited Co on $\mathrm{Au}(111)$ is estimated to be negligible. 
4. SP-STM 


\section{Chapter 5}

\section{Summary \& Outlook}

Electrochemistry offers the possibility of fabricating magnetic structures in fast and low-cost processes. The magnetic properties of an electrochemically deposited material depend on the parameters influencing the growth process, such as the deposition potential and total deposition time. Especially the magnetic properties of ultrathin magnetic films are correlated with their coverage. The magnetic properties of such surfaces can be investigated in-situ at the solid/liquid interface by techniques integrating over a certain surface area such as magneto-optical Kerr effect measurements and alternating gradient field magnetometry. In contrast, spin-polarized scanning tunneling microscopy (sp-STM) at the solid/vacuum interface has already proven its high local resolution of both sample magnetization as well as sample structure. The surface topography in an electrochemical environment can be already investigated by an electrochemical STM (ec-STM) in-situ. This was the point of departure of that thesis with the goal of combining these two techniques for enabling sp-STM at the solid/liquid interface.

One part of this thesis was to modify the present ec-STM setup to enable spSTM in differential magnetic mode sensitive to magnetized samples in out-of-plane direction. For this, a modulated magnetic field with a frequency much higher than the cut-off frequency of the STM setup was required to manipulate the tip magnetization periodically in direction of the surface normal. Such a magnetic field was generated by a self-made small coil which was magnetically shielded, electrically grounded, and permanently installed at the STM scanner unit positioned around the tip end. Special attention was paid to the ferromagnetic tip material which should own a narrow magnetic hysteresis loop as well as vanishing magnetostriction. The magnetic properties of various tip materials were investigated by SQUID and two kinds of modified STM setups to observe the magnetostriction directly. Thereby, magnetostriction could be resolved even if the induced length variation was in the range of an atomic step edge, since a STM setup is highly sensitive to the tip-sample separation. Taking this considerations into account, it could has been 


\section{SUMMARY \& OUTLOOK}

shown that magnetic tips made of $\mathrm{Co}_{68.2} \mathrm{Fe}_{4.3} \mathrm{Si}_{12.5} \mathrm{~B}_{15}$ (AC20) fit best for sp-STM in the differential magnetic mode.

Electrochemically deposited $\mathrm{Co}$ on an $\mathrm{Au}(111)$ single crystal was chosen as a suitable model system for sp-STM at the solid/liquid interface, since ultrathin films of Co deposited on $\mathrm{Au}(111)$ are out-of plane magnetized caused by the magnetocrystalline anisotropy.

The electrochemical deposition of $\mathrm{Co}$ on $\mathrm{Au}(111)$ out of an electrolyte solution composed of sodium sulfate, sulfuric acid, and cobalt sulfate was also an important part of this work, since an electrochemically stable and metallic sample was required. The in-situ deaeration of the electrolyte solution ensured an environment nearly free of dissolved oxygen. Additionally, it was necessary to adjusted the $\mathrm{pH}$-value $\approx 3$ of the supporting electrolyte using sulfuric acid to avoid the formation of Co hydroxides. Although a higher concentration of sulfuric acid would cause a more acidic $\mathrm{pH}$-value, the correlated stronger hydrogen evolution would hinder the Co deposition.

It has been shown by ec-STM investigations, that the observed Co deposition at the solid/liquid interface occurred initially by lateral growth of bilayer islands originating from $\mathrm{Au}(111)$ step edges. Additional Co layers were deposited on top before the initial Co bilayer was coalesced and completed. The deposited Co remained stable on $\mathrm{Au}(111)$ in a potential range from $-450 \mathrm{mV}$ to $-330 \mathrm{mV}$. The high lateral resolution of the STM measurements at the solid/liquid interface was demonstrated by the observed herringbone reconstructed $\mathrm{Au}(111)$ surface and the moiré structure on top of the deposited Co islands. An imported aspect of STM investigations at the solid/liquid interface was the adjustment of a low bias voltage in the range of $20 \mathrm{mV}$ as well as high tunneling currents in the range of $5 \mathrm{nA}$ to ensure a vacuumlike gap between tip and substrate. After the electrochemical sample preparation, extended Co islands up to 4 atomic layers were deposited with bare $\mathrm{Au}(111)$ areas in between. This way, a sample surface was prepared which showed ferromagnetic $\mathrm{Co}$ areas and diamagnetic $\mathrm{Au}$ areas and was therefore an ideal sample for sp-STM at the solid/liquid interface.

The final part of this work were the investigations on $\mathrm{Co} / \mathrm{Au}(111)$ by sp-STM at the solid/liquid interface using AC20 tips. Thereby, the observed sample topography showed a high lateral resolution and was without spurious contributions from the sp-STM setup, since the time averaged tunneling current was not affected. The corresponding spin-polarized component of the tunneling current was recorded by lock-in technique and displayed a sharp contrast between Co and Au areas. This difference in signal was caused by the different spin-polarizations of the sample $P_{\text {sample }, C o} \neq P_{\text {sample }, A u}=0$. This way, it was demonstrated, that sp-STM at the solid/liquid interface was accomplished, since the tunneling process occurred across a vacuum-like gap and the electron spin was conserved. Control experiments were 
performed using a non-ferromagnetic Au tip under similar conditions. Such control measurements showed no contrast between $\mathrm{Co}$ and $\mathrm{Au}$, as it was expected for the tip spin-polarization $P_{t i p, A C 20} \neq P_{t i p, A u}=0$.

The sp-STM investigations on $\mathrm{Co} / \mathrm{Au}(111)$ were completed with sp-STM measurements observed by lock-in technique at the second harmonic. Here, a much smaller difference in signal was observed between $\mathrm{Co}$ and $\mathrm{Au}$ areas. It was assumed that magnetic stray fields of the magnetic tip or the coil of the sp-STM caused an additional modulation of the sample magnetization generating the observed signal at the second harmonic. Since this signal was $\sim 25$ times smaller compared to the magnitude of the spin-polarized signal at the first harmonic, a strong influence of the sp-STM setup on deposited $\mathrm{Co}$ on $\mathrm{Au}(111)$ was negligible.

In summary it was shown, that sp-STM was accomplished in an electrochemical environment. Using $\mathrm{Co} / \mathrm{Au}(111)$ as model system, not only sample topography was investigated but also sample magnetization was observed in-situ.

The slightly different spin-polarized tunneling currents in dependence on the actual Co layer thickness gave a hint to differently aligned Co magnetization $\vec{M}$ with respect to the surface normal. More investigations in detail are required to make a clear statement about the spin reorientation transition from out-of-plane magnetization to in-plane magnetization for ultrathin Co films. Especially, investigations in dependence on the size of the Co islands until the initial Co bilayer is completed as well as in dependence on the Co layer thickness will by of interest.

Based on this work, the magnetization of other magnetic layers or structures prepared in an electrochemical environment can be investigated in-situ with high localized resolution. 
5. SUMMARY \& OUTLOOK 


\section{Appendix A}

\section{Lists of Chemicals, Materials and Devices}

\begin{tabular}{lcccc}
\hline \hline Chemicals & Molecular Formula & Producer & Denotation & Item No. \\
\hline Sodium sulfate & $\mathrm{Na}_{2} \mathrm{SO}_{4}$ & Merck & Suprapur & \\
\hline Cobalt(II) sulfate & $\mathrm{CoSO}_{4}$ & Merck & Suprapur & \\
\hline Sulfuric acid & $\mathrm{H}_{2} \mathrm{SO}_{4}$ & Merck & Suprapur & \\
\hline $\begin{array}{l}\text { Sulfuric acid, 95- } \\
97 \%\end{array}$ & $\mathrm{H}_{2} \mathrm{SO}_{4}$ & Merck & EMPARTA & 1.00731 .2511 \\
Hydrochloric acid & $\mathrm{HCl}$ & Merck & & \\
\hline $\begin{array}{l}\text { Hydrogen perox- } \\
\text { ide, } 35 \%\end{array}$ & $\mathrm{H}_{2} \mathrm{O}_{2}$ & Merck & EMPROVE & 1.08600 .2500 \\
\hline \hline
\end{tabular}

Table A.1: List of chemicals 


\begin{tabular}{|c|c|}
\hline Material & Producer \\
\hline Gold wire $\varnothing 250 \mu \mathrm{m}$ & Merck \\
\hline Nickel bath, Ni Sulfamat HS & Atotech \\
\hline Nickel wire $\varnothing 250 \mu \mathrm{m}$ & Puratronic@99.994\% Alfa Aesar \\
\hline AC20 wire $\varnothing 125 \mu \mathrm{m}$ & $\mathrm{Co}_{68.2} \mathrm{Fe}_{4.3} \mathrm{Si}_{12.5} \mathrm{~B}_{15}$, Unitika LTD. \\
\hline $\begin{array}{l}\mathrm{Au}(111) \text { single crystal } \\
\varnothing 10 \mathrm{~mm} \times 4 \mathrm{~mm}\end{array}$ & MaTecK, ch-Nr.09050614 \\
\hline Gold arrandee $11 \times 11 \mathrm{~mm}$ & arrandee $^{\mathrm{TM}}$, precious metal coated substrates \\
\hline
\end{tabular}

Table A.2: List of materials

\begin{tabular}{ll}
\hline \hline Device & Company, Designation \\
\hline STM & NanoScope E \\
\hline Bipotentiostat & EC-Tec, BP-700 \\
\hline Biscangenerator & EC-Tec \\
\hline Oscilloscope & Tektronix TDS 420A \\
\hline Function/Arbitrary Waveform Generator & Agilent 33220A \\
\hline Lock-in amplifier & EG\&G Princeton Applied Research \\
\hline Teslameter & Model 5209 \\
\hline & Group3 DTM 141 digital teslameter \\
\hline Saturated calomel electrode (SCE) & Group3 Hall Probe MPT-231-2m \\
\hline \hline
\end{tabular}

Table A.3: List of devices 


\section{Appendix B}

\section{Abbreviations}

\begin{tabular}{ll}
\hline \hline Abbreviations & \\
\hline $\mathrm{AL}$ & atomic layer, unit of atomic step height \\
$\AA$ & unit of length $1 \AA=1 \cdot 10^{-10} \mathrm{~m}$ \\
angle $\alpha$ & angle between sample magnetization and surface normal $\vec{a}$ \\
angle $\theta$ & angle between tip magnetization and surface normal $\vec{a}$ \\
$\mathrm{CCM}$ & constant-current-mode, mode of STM operation \\
$C E$ & counter electrode \\
$\mathrm{CHM}$ & constant-height-mode, mode of STM operation \\
$\mathrm{CV}$ & cyclic voltammogram \\
$d$ & tip-substrate distance \\
$E_{0}$ & Nernst potential \\
$E_{00}$ & standard potential \\
ec-STM & scanning tunneling microscopy at the solid/liquid interface \\
$E_{0}\left(M e / M e^{2+}\right)$ & Nernst potential of a metal red/ox couple Me/Me ${ }^{2+}$ \\
$E_{\mathrm{SCE}}$ & potential vs SCE \\
$E_{\mathrm{SHE}}$ & potential vs SHE \\
$E_{\mathrm{tip}}$ & potential of the tip \\
$\mathrm{E}_{\mathrm{WE}}$ & potential of the substrate \\
$f_{\mathrm{mod}}$ & modulation frequency \\
$f_{\mathrm{STM}}$ & scan rate of the STM in $\frac{\text { line }}{\text { second }}$ \\
$f_{\text {cut-off }}$ & cut-off frequency of the STM feedback loop \\
$\hbar$ & Planck constant over $2 \pi$ \\
$I_{0}$ & set point of the tunneling current \\
$I_{s p}$ & spin-polarized tunneling current \\
$I_{a}$ & spin-polarized tunneling current in antiparallel orientation \\
$I_{p}$ & spin-polarized tunneling current in parallel orientation \\
&
\end{tabular}




\begin{tabular}{ll}
$j$ & current density \\
$j_{\text {ano }}$ & anodic current density \\
$j_{\text {cat }}$ & current density \\
$m$ & electron mass \\
ML & monolayer, unit of nominal coverage \\
OPD & over potential deposition, bulk deposition \\
$P_{i}$ & spin polarization of $i=$ tip, sample \\
$R E$ & reference electrode \\
SCE & saturated calomel electrode \\
SHE & standard hydrogen electrode \\
sp-STM & spin-polarized scanning tunneling microscopy \\
STM & scanning tunneling microscopy \\
SQUID & superconducting quantum interference device \\
$\mathrm{UPD}$ & under potential deposition \\
$V_{b}$ & bias voltage \\
$W E$ & working electrode \\
$W E_{\text {sub }}$ & working electrode, substrate \\
$W E_{\text {tip }}$ & working electrode, tip \\
$x$ & x-direction \\
$y$ & y-direction \\
$z$ & z-direction \\
\hline$\kappa$ & decay constant in the barrier region \\
$\phi_{\mathrm{UHV}}$ & work function / barrier height in UHV \\
$\phi_{\mathrm{eff}}$ & effective reduced work function / barrier height \\
\hline \hline
\end{tabular}




\section{List of Publication}

- N. Sircar, S. Ahlers, C. Majer, G. Abstreiter and D. Bougeard Interplay between electrical transport properties of GeMn thin films and Ge substrates. Physical Review B, 83(12):125306-, March 2011.

- C. Majer, W. Schindler. Spin-polarized scanning tunneling microscopy at a solid/liquid interface. in preparation

- C. Majer, W. Schindler. STM for magnetostrictions measurements. in preparation 
B. APPENDIX 


\section{References}

[1] R. Allenspach, M. Stampanoni, and A. Bischof. Magnetic domains in thin epitaxial $\mathrm{Co} / \mathrm{Au}(111)$ films. Phys. Rev. Lett., 65(26):3344-3347, December 1990. URL http://link.aps.org/doi/ 10.1103/PhysRevLett.65.3344 78

[2] P. Allongue and F. Maroun. Electrodeposition of Two-dimensional Magnetic Nanostructures on Single Crystal Electrode Surfaces. In G. Staikov, editor, Electrocrystallization in Nanotechnology, pages 217-241. John Wiley and Sons, 2006. 1 $35,36,45,71,72$

[3] P. Allongue and F. Maroun. Metal electrodeposition on single crystal metal surfaces mechanisms, structure and applications. Curr. Opin. Solid State Mater. Sci., 10(3-4):173-181, June 2006. ISSN 13590286. URL http://www.sciencedirect. com/science/article/B6VS5-4NNPB6V-1/2/ ab641e75637557ea4f47946d1de8e30b 35

[4] P. Allongue, L. Cagnon, C. Gomes, A. Gündel and V. Costa. Electrodeposition of $\mathrm{Co}$ and $\mathrm{Ni} / \mathrm{Au}(111)$ ultrathin layers. Part I: nucleation and growth mechanisms from in situ STM. Surf. Sci., 557(1-3):41-56, May 2004. ISSN 0039-6028. URL http://www.sciencedirect. com/science/article/B6TVX-4C1CBX5-3/2/ 6e06dbc3a0b1cfee892b1353a32db7c4 35, 36, 39 41

[5] P. Allongue, F. Maroun, H. F. Jurca, N. Tournerie, G. Savidand, and R. Cortès. Magnetism of electrodeposited ultrathin layers: Challenges and opportunities. Surf. Sci., 603(10-12):1831-1840, June 2009. ISSN 0039-6028. URL http://www.sciencedirect . com/science/article/B6TVX-4VB5JY5-5/2/ c8ced02c06e3b291caf4aace0fe9d5c5 $1,45,72$

[6] H. Angus and E. Neppiras. Nickel-based magnetostrictive alloys for electromechanical transducers. Ultrasonics, 7(3):182-187, July 1969. ISSN 0041-624X. doi: 10.1016/0041-624X(69)90664-7. URL http://www.sciencedirect.com/ science/article/B6TW2-46X8VJR-DW/2/ 6 fa5bf 4c49f 0535591be19e44b9eb98a
[7] N. W. Ashcroft and N. D. Mermin. Solid State Physics. Philadelphia, Saunders College, 1987. ISBN: 0-03-049346-3. 353941

[8] A. Bard, R. Parsons, and J. Jordan. Standard potentials in aqueous solution. Monographs in electroanalytical chemistry and electrochemistry. Marcel Dekker, New York, 1985. URL http: //books .google.de/books?id=fuJV1H18KtEC 7 14 21]

[9] A. J. Bard and L. R. Faulkner. Electrochemical Methods: Fundamentals and Applications. John Wiley and Sons, second edition, 2001. ISBN: 0471-04372-9. 8, 9 11,33

[10] J. Bardeen. Tunnelling from a Many-Particle Point of View. Phys. Rev. Lett., 6(2):57-59, January 1961. URL http://link.aps.org/doi/10. 1103/PhysRevLett.6.57 25$] 26$

[11] J. V. Barth, H. Brune, G. Ertl, and R. J. Behm. Scanning tunneling microscopy observations on the reconstructed $\mathrm{Au}(111)$ surface: Atomic structure, long-range superstructure, rotational domains, and surface defects. Phys. Rev. B, 42(15): 9307-9318, Nov 1990. doi: 10.1103/PhysRevB. 42.9307. 33,38

[12] N. Batina, T. Will, and D. M. Kolb. Study of the initial stages of copper deposition by in situ scanning tunnelling microscopy. Faraday Discuss., 94:-, 1992. ISSN 1359-6640. URL http://dx.doi.org/10.1039/FD9929400093 12

[13] I. Benjamin, D. Evans, and A. Nitzan. Electron tunneling through water layers: Effect of layer structure and thickness. J. Chem. Phys., 106(16):6647-6654, 1997. doi: 10.1063/1. 474079. URL http://link .aip.org/link/?JCP/ 106/6647/1. 30

[14] M. Binggeli, D. Carnal, R. Nyffenegger, H. Siegenthaler, R. Christoph, and H. Rohrer. Electrolytic scanning tunneling microscopy and point contact studies at electrochemically polished $\mathrm{Au}(111)$ substrates with and without $\mathrm{Pb}$ adsorbates. J. Vac. Sci. Technol., B, 9(4):19851992, 1991. doi: 10.1116/1.585393. URL http: //link.aip.org/link/?JVB/9/1985/1 30

[15] G. Binnig and H. Rohrer. Scanning tunneling microscopy. Surf. Sci., 126(13):236-244, 1983. ISSN 0039-6028. doi: 10.1016/0039-6028(83)90716-1. URL http://www.sciencedirect.com/science/ article/pii/0039602883907161 [2] 25

[16] G. Binnig, H. Rohrer, C. Gerber, and E. Weibel. Tunneling through a controllable vacuum gap. Appl. Phys. Lett., 40(2):178-180, 1982. doi: 10.1063/1.92999. URL http://link.aip.org/ link/?APL/40/178/1 25 
[17] G. Binnig, H. Rohrer, C. Gerber, and E. Weibel. Surface Studies by Scanning Tunneling Microscopy. Phys. Rev. Lett., 49(1):57-61, July 1982. URL http://link. aps.org/doi/10.1103/ PhysRevLett.49.57 2] 25

[18] G. Binnig, H. Rohrer, C. Gerber, and E. Weibel. 77 Reconstruction on $\mathrm{Si}(111)$ Resolved in Real Space. Phys. Rev. Lett., 50(2):120-123, January 1983. URL http://link.aps.org/doi/10.1103/ PhysRevLett.50.120 2025

[19] G. Binnig, H. Rohrer, C. Gerber, and E. Weibel. (111) facets as the origin of reconstructed $\mathrm{Au}(110)$ surfaces. Surf. Sci., 131(1):L379-L384, $1983 . \quad$ ISSN 00396028. doi: 10.1016/0039-6028(83)90112-7. URL http://www.sciencedirect.com/science/ article/pii/0039602883901127 2[25

[20] G. Binnig, H. Rohrer, C. Gerber, and E. Weibel. Vacuum tunneling. Physica $B+C$, 109-110(0):2075-2077, 1982. ISSN 03784363. doi: 10.1016/0378-4363(82)90241-8. URL http://www.sciencedirect.com/science/ article/pii/0378436382902418 30

[21] S. Blügel, D. Pescia, and P. H. Dederichs. Ferromagnetism versus antiferromagnetism of the Cr(001) surface. Phys. Rev. B, 39(2):1392-1394, January 1989. URL http://link.aps.org/doi/ 10.1103/PhysRevB.39.1392 49

[22] J. O. Bockris, M. A. V. Devanathan, and K. Muller. On the Structure of Charged Interfaces. Proc. R. Soc. Lond. A, 274(1356): 55-79, 1963. doi: 10.1098/rspa.1963.0114. URL http://rspa.royalsocietypublishing.org/ content/274/1356/55. abstract 8

[23] M. Bode. Spin-polarized scanning tunnelling microscopy. Rep. Prog. Phys., 66(4):523-582, 2003. ISSN 0034-4885. doi: 10.1088/0034-4885/ 66/4/203. URL http://iopscience.iop.org/ $0034-4885 / 66 / 4 / 203,24548$

[24] N. Bohr. I. On the constitution of atoms and molecules. Philosophical Magazine Series 6, 26(151):1-25, 1913. doi: $\quad 10.1080 / 14786441308634955 . \quad$ URL http://www.tandfonline.com/doi/abs/10. 1080/14786441308634955 1

[25] S. G. Bratsch. Standard Electrode Potentials and Temperature Coefficients in Water at $298.15 \mathrm{~K}$. J. Phys. Chem. Ref. Data, 18(1):1-21, 1989. doi: 10.1063/1.555839. URL http://link.aip.org/ link/?JPR/18/1/1 7

[26] R. A. Brizzolara and R. J. Colton. Magnetostriction measurements using a tunneling-tip strain detector. J. Magn. Magn. Mater.,
88(3):343-350, $1990 . \quad$ ISSN $\quad 0304-8853$. doi: $\quad 10.1016 / 0304-8853(90) 90657-C . \quad$ URL http://www.sciencedirect.com/science/ article/pii/030488539090657C 58

[27] P. J. Bryant, H. S. Kim, Y. C. Zheng, and R. Yang. Technique for shaping scanning tunneling microscope tips. Rev. Sci. Instrum., 58(6): 1115-1115, 1987. doi: 10.1063/1.1139618. URL http://link.aip.org/link/?RSI/58/1115/1 31

[28] J. L. Bubendorff, E. Beaurepaire, C. Mény, P. Panissod, and J. P. Bucher. Perpendicular magnetization in ultrathin electrodeposited cobalt films. Phys. Rev. B, 56(12):R7120-R7128, September 1997. URL http://link.aps.org/ doi/10.1103/PhysRevB.56.R7120 1 , 72

[29] J. L. Bubendorff, L. Cagnon, V. Costa-Kieling, J. P. Bucher, and P. Allongue. Anion promoted Ni-underpotential deposition on $\mathrm{Au}(111)$. Surf. Sci., 384(1-3):L836-L843, July 1997. ISSN 0039-6028. URL http://www.sciencedirect. com/science/article/B6TVX-3WOJXCS-4K/2/ e150fe459dd4d59e8ebd467a52054c8d. 72

[30] J. L. Bubendorff, E. Beaurepaire, C. Mény, and J. P. Bucher. Overpotential driven perpendicular magnetization of electrodeposited ultrathin cobalt films. J. Appl. Phys., 83(11):7043-7045, June 1998. URL http://dx.doi.org/10.1063/ 1.367820 . 1

[31] J.-P. Bucher, L. Santesson, and K. Kern. Thermal Healing of Self-Assembled Organic Monolayers: Hexane- and Octadecanethiol on $\mathrm{Au}(111)$ and $\mathrm{Ag}(111)$. Langmuir, 10(4):979-983, 1994. doi: 10.1021/la00016a001. URL http://pubs. acs.org/doi/abs/10.1021/la00016a001.33

[32] E. Budevski, G. Staikov, and W. J. Lorenz. Electrochemical Phase Formation and Growth: An Introduction to the Initial Stages of Metal Deposition. VCH Verlagsgesellschaft mbH, Weinheim, 1996. doi: DOI:10.1002/ 9783527614936. URL http://onlinelibrary. wiley.com/book/10.1002/9783527614936 8

[33] L. Cagnon, A. Gundel, T. Devolder, A. Morrone, C. Chappert, J. Schmidt, and P. Allongue. Anion effect in $\mathrm{Co} / \mathrm{Au}(111)$ electrodeposition: structure and magnetic behavior. Appl. Surf. Sci., 164(1-4):22-28, $2000 . \quad$ ISSN 01694332. doi: 10.1016/S0169-4332(00)00330-5. URL http://www.sciencedirect.com/science/

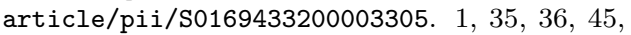
72

[34] L. Cagnon, T. Devolder, R. Cortes, A. Morrone, J. E. Schmidt, C. Chappert, and P. Allongue. Enhanced interface perpendicular magnetic anisotropy in electrodeposited $\mathrm{Co} / \mathrm{Au}(111)$ 
layers. Phys. Rev. B, 63(10):104419, February 2001. URL http://link.aps.org/doi/10.1103/ \begin{tabular}{ll|l|l|l|l|l|} 
PhysRevB.63.104419 & 1 & 35 & 36 & 41 & 72 & 81 \\
\hline
\end{tabular}

[35] P. F. Carcia, A. D. Meinhaldt, and A. Suna. Perpendicular magnetic anisotropy in $\mathrm{Pd} / \mathrm{Co}$ thin film layered structures. Appl. Phys. Lett., 47 (2):178-180, 1985. doi: 10.1063/1.96254. URL http://link.aip.org/link/?APL/47/178/1. 71

[36] M. Cavallini and F. Biscarini. Electrochemically etched nickel tips for spin polarized scanning tunneling microscopy. Rev. Sci. Instrum., 71(12): 4457-4460, 2000. doi: 10.1063/1.1311936. URL http://link. aip.org/link/?RSI/71/4457/1 31

[37] D. L. Chapman. A Contribution to the Theory of Electrocapillarity. The London, Edinburgh, and Dublin Philosophical Magazine and Journal of Science, Ser. 6, Vol. 25:475481, 1913. URL http://electrochem. cwru.edu/ estir/hist/hist-25-Chapman.pdf 8,9

[38] C. Chappert, D. Renard, P. Beauvillain, J. Renard, and J. Seiden. Ferromagnetism of very thin films of nickel and cobalt. J. Magn. Magn. Mater., 54-57(Part 2):795-796, February 1986. ISSN 03048853. URL http://www.sciencedirect. com/science/article/B6TJJ-46JGNSR-FT/2/ c77be09b7fdb22da1a3db268f506fd2e [1]

[39] C. J. Chen. Introduction to Scanning Tunneling Microscopy. Oxford Science Publications, second edition edition, 2008. ISBN: 978-0-19-921150-0. 2533

[40] D.-X. Chen, L. Pascual, F. J. Castaño, A. Hernando, and M. Vazquez. Anomalous asymmetric magneto-inductance in amorphous $\mathrm{Co}_{68.2} \mathrm{Fe}_{4.3} \mathrm{Si}_{12.5} \mathrm{~B}_{15}$ wire with shifted hysteresis loop. J. Phys. D: Appl. Phys., 33(2):111-114, 2000. ISSN 0022-3727. URL http://stacks . iop. org $/ 0022-3727 / 33 / i=2 / a=303$. 68

[41] R. Christoph, H. Siegenthaler, H. Rohrer, and $\mathrm{H}$. Wiese. In situ scanning tunneling microscopy at potential controlled $\mathrm{Ag}(100)$ substrates. Electrochim. Acta, 34(8):1011-1022, 1989. ISSN 0013-4686. doi: 10.1016/0013-4686(89)87134-8. URL http://www.sciencedirect.com/science/ article/pii/0013468689871348 25$] 29$

[42] J. L. Costa, J. Nogues, and K. V. Rao. Direct measurements of magnetostrictive process in amorphous wires using scanning tunneling microscopy. Appl. Phys. Lett., 66(24):3374-3376, 1995. doi: 10.1063/1.113762. URL http://link. aip.org/link/?APL/66/3374/1 [58
[43] A. Cuesta, M. Kleinert, and D. M. Kolb. The adsorption of sulfate and phosphate on $\mathrm{Au}(111)$ and $\mathrm{Au}(100)$ electrodes: an in situ STM study. Phys. Chem. Chem. Phys., 2(24):5684-5690, 2000. URL http://dx.doi.org/10.1039/b006464p 15 $1 6 \longdiv { 2 5 } 3 3$

[44] A. S. Dakkouri. Reconstruction phenomena at gold/electrolyte interfaces: an in-situ STM study of $\mathrm{Au}(100)$. Solid State Ionics, 94(1-4):99-114, February 1997. ISSN 0167-2738. URL http://www.sciencedirect. com/science/article/B6TY4-3VSNK93-68/2/ 1c76aee4ba805a2ef7727e0cac5b9abd 25

[45] L. Diekhöner, M. A. Schneider, A. N. Baranov, V. S. Stepanyuk, P. Bruno, and K. Kern. Surface States of Cobalt Nanoislands on $\mathrm{Cu}(111)$. Phys. Rev. Lett., 90(23):236801, June 2003. URL http://link.aps.org/doi/10.1103/ PhysRevLett.90.236801 45

[46] H. F. Ding, W. Wulfhekel, C. Chen, J. Barthel, and J. Kirschner. A combined magnetic force and spin-polarized scanning tunneling microscopy study of the closure domain pattern of $\mathrm{Co}(0001)$ Mater. Sci. Eng. $B$, 84(1-2):96-101, July 2001. ISSN 09215107. URL http://www. sciencedirect . com/science/article/B6TXF-435MFHT-K/2/ bf57aaaa457e87c81def75f1bf0b38af $45,69,71$

[47] H. F. Ding, W. Wulfhekel, and J. Kirschner. Ultra sharp domain walls in the closure domain pattern of $\mathrm{Co}(0001)$. EPL (Europhysics Letters), 57 (1):100-106, 2002. ISSN 0295-5075. URL http: //stacks. iop.org/0295-5075/57/i=1/a=100. 78

[48] H. F. Ding, W. Wulfhekel, J. Henk, P. Bruno, and J. Kirschner. Absence of Zero-Bias Anomaly in Spin-Polarized Vacuum Tunneling in $\mathrm{Co}(0001)$. Phys. Rev. Lett., 90(11):116603, March 2003. doi: 10.1103/PhysRevLett.90. 116603. URL http://link.aps.org/doi/10. 1103/PhysRevLett.90.116603 78

[49] H. Ding. Spin-polarized scanning tunneling microscopy. $\quad \mathrm{PhD}$ thesis, MathematischNaturwissenschaftlich-Technischen Fakultät (mathematisch-naturwissenschaftlicher Bereich) der Martin-Luther-Universität HalleWittenberg, 2001. URL http://sundoc. bibliothek.uni-halle.de/diss-online/01/ 01H155/prom.pdf 558285

[50] B. Drake, R. Sonnenfeld, J. Schneir, and P. Hansma. Scanning tunneling microscopy of processes at liquid-solid interfaces. Surf. Sci., 181(12):9-97, 1987. ISSN 0039-6028. doi: 10.1016/0039-6028(87)90144-0. URL http://www.sciencedirect.com/science/ article/pii/0039602887901440 29 
[51] G. J. Edens, X. Gao, and M. J. Weaver. The adsorption of sulfate on gold(111) in acidic aqueous media: adlayer structural inferences from infrared spectroscopy and scanning tunneling microscope. J. Electroanal. Chem., 375(1-2):357-366, September 1994. ISSN 0022-0728. URL http://www.sciencedirect. com/science/article/B6TGB-44DTH1B-DR/2/ 45cfa5d9bdd86c946485846df06a5936. 20, 15,16 33

[52] N. Ekreem, A. Olabi, T. Prescott, A. Rafferty, and M. Hashmi. An overview of magnetostriction, its use and methods to measure these properties. J. Mater. Process. Tech., 191(1-3):96-101, August 2007. ISSN 09240136. URL http://www.sciencedirect. com/science/article/B6TGJ-4N7SBMV-3/2/ 5898f6eb1e422f5426b5462d7a205778 5458

[53] G. E. Engelmann, J. C. Ziegler, and D. M. Kolb. Electrochemical fabrication of large arrays of metal nanoclusters. Surf. Sci., 401(2):L420-L424, April 1998. ISSN 00396028. URL http://www.sciencedirect. com/science/article/B6TVX-3V6X695-3/2/ 3fa4716a35804ff090e763d552de8d4d, 30

[54] Faraday. Ueber electrisch-magnetische Bewegungen, und die Theorie des Magnetismus. Annalen der Physik, 71(6): $124-171, \quad 1822 . \quad$ ISSN $1521-3889$. doi: 10.1002/andp.18220710603. URL http: //dx.doi.org/10.1002/andp.18220710603 1

[55] J. Ferré, G. Pénissard, C. Marlière, D. Renard, P. Beauvillain, and J. P. Renard. Magnetooptical studies of $\mathrm{Co} / \mathrm{Au}$ ultrathin metallic films. Applied Physics Letters, 56(16):1588-1590, 1990. doi: 10.1063/1.103136. URL http://link.aip. org/link/?APL/56/1588/1 1

[56] H. Fujimori, K. I. Arai, H. Shirae, H. Saito, T. Masumoto, and N. Tsuya. Magnetostriction of Fe-Co Amorphous Alloys. Jpn. J. Appl. Phys., 15(4):705-706, 1976. doi: 10.1143/JJAP.15.705. URL http://jjap.jsap.jp/link?JJAP/15/705/ 54

[57] W. Gale, S. Newcomb, T. Totemeier, and G. V. Voort. Metallography. In W. F. Gale and T. C. Totemeier, editors, Smithells Metals Reference Book, pages 10-1 to 10-87. Elsevier Science \& Technology, eighth edition edition, 2003. ISBN: 978-0-7506-7509-3. 31

[58] M. Galperin, A. Nitzan, and I. Benjamin. Numerical Simulations of Electron Tunneling Currents in Water. J. Phys. Chem. A, 106(45):10790-10796, 2002. doi: 10.1021/ jp025813j. URL http://pubs.acs.org/doi/abs/ 10.1021/jp025813j 30

[59] E. Garcia, J. Santos, E. Pereira, and M. Freitas. Electrodeposition of cobalt from spent Li-ion battery cathodes by the electrochemistry quartz crystal microbalance technique. $J$. Power Sources, 185(1):549-553, 2008. ISSN 0378-7753. doi: 10.1016/j.jpowsour.2008.07.011. URL http://www.sciencedirect.com/science/ article/pii/S0378775308013864 17 |21

[60] N. Garcia. Theory of scanning tunneling microscopy and spectroscopy: Resolution, image and field states, and thin oxide layers. IBM J. Res. Dev., 30(5):533-542, 1986. ISSN 0018-8646. doi: 10.1147/rd.305. 0533. URL http://ieeexplore.ieee.org/xpl/ articleDetails .jsp?arnumber $=5390154,[30$

[61] C. F. Gauss. Die Intensität der erdmagnetischen Kraft zurückgeführt auf absolutes Maass. Annalen der Physik, 104(8):591-615, 1833. ISSN 1521-3889. doi: 10.1002/andp. 18331040808. URL http://dx.doi.org/10. 1002/andp.18331040808. 1

[62] H. Gerischer, D. Kolb, and J. Sass. The study of solid surfaces by electrochemical methods. Adv. Phys., 27(3):437-498, 1978. doi: $\quad 10.1080 / 00018737800101424$. URL http://www.tandfonline.com/doi/abs/10. 1080/00018737800101424 2 [5

[63] A. A. Gewirth and B. K. Niece. Electrochemical Applications of in Situ Scanning Probe Microscopy. Chem. Rev., 97(4):1129-1162, 1997. doi: 10.1021/cr960067y. URL http://pubs. acs.org/doi/abs/10.1021/cr960067y PMID: 11851445. 2, 25

[64] A. A. Gewirth, D. H. Craston, and A. J. Bard. Fabrication and characterization of microtips for in situ scanning tunneling microscopy. J. Electroanal. Chem., 261(2, Part 2):477-482, 1989. ISSN 0022-0728. doi: 10.1016/0022-0728(89) 85018-1. URL http://www. sciencedirect.com/ science/article/pii/0022072889850181] 33

[65] J. K. Gimzewski and R. Möller. Transition from the tunneling regime to point contact studied using scanning tunneling microscopy. Phys. Rev. B, 36:1284-1287, Jul 1987. doi: 10.1103/ PhysRevB.36.1284. URL http://link.aps.org/ doi/10.1103/PhysRevB.36.1284]30

[66] J. Gimzewski, R. Möller, D. Pohl, and R. Schlittler. Transition from tunneling to point contact investigated by scanning tunneling microscopy and spectroscopy. Surf. Sci., 189-190:15-23, October 1987. ISSN 00396028. URL http://www.sciencedirect. 
com/science/article/B6TVX-4KKKS3T-9/2/ a78634b9bb79b57bb7341c41a2ca3b05 PH: 169. 30

[67] L. G. Gouy. Sur la constitution de la charge électrique à la surface d'un électrolyte. Comptes rendus hebdomadaires des séances de l'Académie des sciences, 149:654-657, 1909. URL http://gallica.bnf.fr/ark: /12148/bpt6k3103r/f654.image [8, 9

[68] L. G. Gouy. Sur la constitution de la charge électrique à la surface d'un électrolyte. Journal de Physique Théorique et Appliquée, Ser. 4, Vol. 9:457-468, 1910. URL http://electrochem. cwru.edu/estir/hist/hist-21-Gouy-1.pdf. 8

[69] U. Gradmann and J. Mller. Flat Ferromagnetic, Epitaxial $48 \mathrm{Ni} / 52 \mathrm{Fe}(111)$ Films of few Atomic Layers. Phys. Status Solidi B, 27(1): 313-324, 1968. ISSN 1521-3951. doi: 10.1002/ pssb.19680270133. URL/http://dx.doi.org/10. 1002/pssb.19680270133 71

[70] D. C. Grahame. The Electrical Double Layer and the Theory of Electrocapillarity. Chem. Rev., 41(3):441-501, 1947. doi: 10.1021/ cr60130a002. URL http://pubs.acs.org/doi/ abs/10.1021/cr60130a002 8

[71] M. P. Green, K. J. Hanson, D. A. Scherson, X. Xing, M. Richter, P. N. Ross, R. Carr, and I. Lindau. In situ scanning tunneling microscopy studies of the underpotential deposition of lead on gold (111). J. Phys. Chem., 93(6):2181-2184, 1989 . doi: 10.1021/ j100343a001. URL http://pubs.acs.org/doi/ abs/10.1021/j100343a001]29

[72] D. Grujicic and B. Pesic. Electrochemical and AFM study of cobalt nucleation mechanisms on glassy carbon from ammonium sulfate solutions. Electrochim. Acta, 49(26):4719-4732, 2004. ISSN 0013-4686. doi: 10.1016/j.electacta.2004.05.028. URL http://www.sciencedirect.com/science/ article/pii/S0013468604005390 21

[73] A. Gündel, L. Cagnon, C. Gomes, A. Morrone, J. Schmidt, and P. Allongue. In-situ magnetic measurements of electrodeposited ultrathin Co, $\mathrm{Ni}$ and $\mathrm{Fe} / \mathrm{Au}(111)$ layers. Phys. Chem. Chem. Phys., 3(16):3330-3335, 2001. URL http://dx. doi.org/10.1039/b100547m. 1, 457281

[74] T. Hachiya, H. Honbo, and K. Itaya. Detailed underpotential deposition of copper on gold(111) in aqueous solutions. J. Electroanal. Chem., 315(1-2):275-291, 1991. ISSN 0022-0728. doi: 10.1016/0022-0728(91)80076-3. URL http://wWw.sciencedirect.com/science/ article/pii/0022072891800763. 12
[75] J. Hahn, Y. Hong, and H. Kang. Electron tunneling across an interfacial water layer inside an STM junction: tunneling distance, barrier height and water polarization effect. Appl. Phys. A, 66:S467-S472, 1998. ISSN 0947-8396. URL http://dx.doi.org/10.1007/ s003390051184 10.1007/s003390051184. 30

[76] J. Halbritter. Tunnel channels, spectroscopy and imaging in STM. Appl. Phys. A, 66: S181-S186, 1998. ISSN 0947-8396. URL http://dx.doi.org/10.1007/s003390051126 10.1007/s003390051126. 30

[77] J. Halbritter, G. Repphun, S. Vinzelberg, G. Staikov, and W. Lorenz. Tunneling mechanisms in electrochemical STM - distance and voltage tunneling spectroscopy. Electrochim. Acta, 40(10):1385-1394, $1995 . \quad$ ISSN 00134686. doi: 10.1016/0013-4686(95)00038-G. URL http://www.sciencedirect.com/science/ article/pii/001346869500038G Electeochemistry. 30

[78] C. H. Hamann and W. Vielstich. Elektrochemie. WILEY-VCH Verlag GmbH \& Co. KGaA, weinhaim, 4. edition, 2005. 6, 7, 8

[79] J. R. Hampton, J.-L. Martinez-Albertos, and H. D. Abruna. Development of a versatile SMOKE system with electrochemical applications. Rev. Sci. Instrum., 73(8):3018-3021, 2002. doi: 10.1063/1.1490416. URL http://link .aip. org/link/?RSI/73/3018/1 72

[80] J. R. Hampton, J.-L. Martnez-Albertos, and H. D. Abrua. SMOKE Studies of Electrodeposited Mono- and Multilayers. Langmuir, 19(10):4309-4315, 2003 . doi: 10.1021/ la020761a. URL/http://pubs.acs.org/doi/abs/ 10.1021/1a020761a 72

[81] W. Heisenberg. Zur Theorie des Ferromagnetismus. Zeitschrift fr Physik, 49:619-636, 1928. ISSN 0044-3328. doi: 10.1007/BF01328601. URL http://dx.doi.org/10.1007/BF01328601 1

[82] H. Helmholtz. Ueber einige Gesetze der Vertheilung elektrischer Ströme in körperlichen Leitern mit Anwendung auf die thierischelektrischen Versuche. Ann. Phys., 165(6):211233, 1853. ISSN 1521-3889. doi: 10.1002/ andp.18531650603. URL http://dx.doi.org/ 10.1002/andp. 18531650603 . 8

[83] H. Helmholtz. Ueber einige Gesetze der Vertheilung elektrischer Ströme in körperlichen Leitern, mit Anwendung auf die thierischelektrischen Versuche (Schluss.). Ann. Phys., 165 (7):353-377, 1853. ISSN 1521-3889. doi: 10. 1002/andp.18531650702. URL http://dx.doi. org/10.1002/andp.18531650702 8 
[84] H. Helmholtz. Studien über electrische Grenzschichten. Ann. Phys., 243(7):337-382, 1879. ISSN 1521-3889. doi: 10.1002/andp. 18792430702. URL http://dx.doi.org/10. 1002/andp.18792430702 8

[85] M. A. Henderson. The interaction of water with solid surfaces: fundamental aspects revisited. Surf. Sci. Rep., 46(18):1-308, 2002. ISSN 0167-5729. doi: 10.1016/S0167-5729(01)00020-6. URL http://www.sciencedirect.com/science/ article/pii/S0167572901000206 [30]

[86] A. Hickling. Studies in electrode polarisation. Part IV.-The automatic control of the potential of a working electrode. Trans. Faraday Soc., 38:27-33, 1942. ISSN 0014-7672. URL http: //dx.doi.org/10.1039/TF9423800027 10

[87] D. Hillel. Water, properties. In E. in Chief: Daniel Hillel, editor, Encyclopedia of Soils in the Environment, pages 290 - 300. Elsevier, Oxford, 2005. ISBN 978-0-12-3485304. doi: 10.1016/B0-12-348530-4/00442-2. URL http://www.sciencedirect.com/science/ article/pii/B0123485304004422. 9

[88] M. H. Hölzle, V. Zwing, and D. M. Kolb. The influence of steps on the deposition of $\mathrm{Cu}$ onto $\mathrm{Au}(111)$ Electrochim. Acta, 40(10):1237-1247, July 1995. ISSN 00134686. URL http://www.sciencedirect. com/science/article/B6TG0-3YS8B4S-6C/2/ 3f885987a89ca01be953787a8ece8116 2]

[89] M. Hölzle, U. Retter, and D. Kolb. The kinetics of structural changes in $\mathrm{Cu}$ adlayers on $\mathrm{Au}(111)$. J. Electroanal. Chem., 371(1-2):101-109, June 1994. ISSN 00220728. URL http://www.sciencedirect. com/science/article/B6TGB-44XDG34-H4/2/ 92334a4ce2aa18f 1359fee6f814bdb2c. 12

[90] Y. A. Hong, J. R. Hahn, and H. Kang. Electron transfer through interfacial water layer studied by scanning tunneling microscopy. J. Chem. Phys., 108(11):4367-4370, 1998. doi: 10.1063/1. 475847. URL/http://link.aip.org/link/?JCP/ 108/4367/1 30

[91] H. Hopster and H. P. Oepen, editors. Magnetic Microscopy of Nanostructures. NanoScience and Technology. Springer Berlin, Heidelberg, 2005. ISBN 978-3-540-40186-5. 48

[92] M. Hugelmann. Erzeugung und Charakterisierung metallischer Nanostrukturen auf nSi(111):H Einkristalloberflächen. PhD thesis, Fakultät für Elektrotechnik und Informationstechnik der Universität Fridericiana Karlsruhe, 2004. URL http://digbib.ubka. uni-karlsruhe.de/volltexte/1000001173 [11 30,78
[93] M. Hugelmann and W. Schindler. Tunnel barrier height oscillations at the solid/liquid interface. Surf. Sci., 541(1-3): L643-L648, September 2003. ISSN 00396028. URL http://www.sciencedirect. com/science/article/B6TVX-4950PPB-1/2/ 39a7d2d2f0f c58265814dee849a55b14 PH: 088. $3 0 \longdiv { 3 8 } 7 8$

[94] P. Hugelmann. Elektrochemische Herstellung und Charakterisierung von Nanostrukturen - Entwicklung ultrascharfer STM-Spitzen als Nanoelektroden. $\mathrm{PhD}$ thesis, Fakultät für Elektrotechnik und Informationstechnik der Universität Fridericiana Karlsruhe, 2004. URL http://digbib.ubka.uni-karlsruhe.de/ volltexte/1000001689 11]31

[95] K. Itaya and E. Tomita. Scanning tunneling microscope for electrochemistry - a new concept for the in situ scanning tunneling microscope in electrolyte solutions. Surf. Sci., 201(3):L507-L512, $1988 . \quad$ ISSN 00396028. doi: 10.1016/0039-6028(88)90489-X. URL http://www.sciencedirect.com/science/ article/pii/003960288890489X 29

[96] S. Jakob and W. Schindler. Electric field assisted electrochemical writing of Co nanostructures onto n-Si(111):H surfaces. Surface Science, (0):-, 2013. ISSN 0039-6028. doi: 10.1016/j.susc.2013. 02.002. URL http://www.sciencedirect.com/ science/article/pii/S0039602813000411 2

[97] R. Jansen, M. Speckmann, H. Oepen, and H. van Kempen. Morphology and magnetism of thin Co films on textured Au surfaces. J. Magn. Magn. Mater., 165(13):258-261, 1997. ISSN 03048853. doi: 10.1016/S0304-8853(96)00523-9. URL http://www.sciencedirect.com/ science/article/pii/S0304885396005239 ¡ce:title¿Symposium E: Magnetic Ultrathin Films, Multilayers and Surfacesi/ce:titlej. 1, 71

[98] M. Johnson and J. Clarke. Spin-polarized scanning tunneling microscope: Concept, design, and preliminary results from a prototype operated in air. J. Appl. Phys., 67(10):6141-6152, May 1990. URL http://link.aip.org/link/?JAP/ 67/6141/1 49

[99] S. Jomni, N. Mliki, R. Belhi, K. Abdelmoula, M. Ayadi, and G. Nihoul. Face centred cubic cobalt ultrathin-layers in $\mathrm{Au} / \mathrm{Co}(111)$ multilayers: a study by electron diffraction and by HREM. Thin Solid Films, $370(12): 186-191, \quad 2000 . \quad$ ISSN 0040-6090. doi: $10.1016 /$ S0040-6090(00)00916-0. URL http://www.sciencedirect.com/science/ article/pii/S0040609000009160] [35 
[100] M. Julliere. Tunneling between ferromagnetic films. Phys. Lett. A, 54(3): 225-226, September 1975. ISSN 03759601. URL http://www.sciencedirect. com/science/article/B6TVM-46R3N46-10D/2/ 90703cfc684b0679356dce9a76b2e942 46

[101] K. Jüttner. Oxygen reduction electrocatalysis by underpotential deposited metal atoms at different single crystal faces of gold and silver. Electrochim. Acta, 29(11): 1597-1604, November 1984. ISSN 00134686. URL http://www.sciencedirect. com/science/article/B6TG0-44CYN76-7X/2/ 70de18fde1ca229a6a837d19cf6499bf PH: 173. 13

[102] M. Kleinert, H. F. Waibel, G. E. Engelmann, H. Martin, and D. M. Kolb. Co deposition on $\mathrm{Au}(111)$ and $\mathrm{Au}(100)$ electrodes: an in situ STM study. Electrochim. Acta, 46 (20-21):3129-3136, July 2001. ISSN 00134686. URL http://wWw.sciencedirect. com/science/article/B6TG0-43RJWW4-C/2/ d8ce14524f82d645fd4bafda757f0f30 $2 2 \longdiv { 3 5 }$

[103] O. Kohmoto, N. Yamaguchi, K. Ohya, and H. Fujishima. Change of the Magnetic Property in Some Amorphous Alloys by Low Temperature Annealing. Jpn. J. Appl. Phys., 17(1):257-258, 1978. doi: 10.1143/JJAP.17.257. URL http: //jjap.jsap.jp/link?JJAP/17/257/ 54

[104] D. M. Kolb. Reconstruction phenomena at metal-electrolyte interfaces. Prog. Surf. Sci., 51(2):109-173, February 1996. ISSN 0079-6816. URL http://www.sciencedirect. com/science/article/B6TJF-3VTC33K-1/2/ 498485efa44107106b449e9000449af3 [1],15, 16 33

[105] D. M. Kolb and J. Schneider. Surface reconstruction in electrochemistry: $\mathrm{Au}(100)-(5 \times 20)$, $\mathrm{Au}(111)-(1 \times 23)$ and $\mathrm{Au}(110)-(1 \times 2)$. Electrochim. Acta, 31(8):929-936, $1986 . \quad$ ISSN 0013-4686. doi: $\quad$ DOI:10.1016/0013-4686(86)80005-6. URL http://www.sciencedirect.com/ science/article/B6TG0-44XVY18-8S/2/ 90c79faedf86a4b15facc92d9beb0ba3 33

[106] D. M. Kolb. Electrochemical Surface Science. Angew. Chem., Int. Ed., 40(7): $1162-1181,2001 . \quad$ ISSN 1521-3773. doi: 10.1002/1521-3773(20010401)40:7〈1162:: AID-ANIE1162 3.0.CO;2-F. URL http:// dx.doi.org/10.1002/1521-3773(20010401)40: 7<1162: :AID-ANIE1162>3.0.CO;2-F. 8,9$] 29$

[107] D. M. Kolb. Elektrochemische Oberflächenphysik. Angew. Chem., 113(7):1198$1220,2001 . \quad$ ISSN 1521-3757. doi:
10.1002/1521-3757(20010401)113:7〈1198: AID-ANGE1198>3.0.CO;2-N. URL http:// dx.doi.org/10.1002/1521-3757(20010401)113: 7<1198: :AID-ANGE1198>3.0.CD;2-N 29

[108] D. Kolb. An atomistic view of electrochemistry. Surf. Sci., 500(1-3):722-740, 2002. ISSN 00396028. doi: DOI:10.1016/S0039-6028(01)01583-7. URL http://www.sciencedirect.com/ science/article/B6TVX-446V8P9-4/2/ 9841007267682a5fb58ccfabfdf4ec11] 2] 13

[109] O. Kongstein, G. Haarberg, and J. Thonstad. Current efficiency and kinetics of cobalt electrodeposition in acid chloride solutions. Part I: The influence of current density, $\mathrm{pH}$ and temperature. J. Appl. Electrochem., 37:669-674, 2007. ISSN 0021-891X. URL http://dx.doi.org/10. 1007/s10800-007-9299-z $10.1007 / \mathrm{s} 10800-007-$ 9299-z. 1] 21

[110] T. Koop, W. Schindler, A. Kazimirov, G. Scherb, J. Zegenhagen, T. Schulz, R. Feidenhans'l, and J. Kirschner. Electrochemical cell for in situ xray diffraction under ultrapure conditions. Rev. Sci. Instrum., 69(4):1840-1843, 1998. doi: 10. 1063/1.1148852. URL http://link.aip.org/ link/?RSI/69/1840/1 PH: 174. 13

[111] Y. Kuk and P. J. Silverman. Scanning tunneling spectroscopy of metal surfaces. J. Vac. Sci. Technol., A, 8(1):289-292, 1990. doi: 10.1116/1. 577087. URL http://link . aip.org/link/?JVA/ 8/289/1. 30

[112] E. W. Lee. Magnetostriction and Magnetomechanical Effects. Rep. Prog. Phys., 18(1):184229,1955 . ISSN 0034-4885. doi: 10.1088/ 0034-4885/18/1/305. URL http://www.iop. org/EJ/abstract/0034-4885/18/1/305/ 54

[113] D. R. Lide, editor. CRC Handbook of Chemistry and Physics. Crc Pr Inc, 87th edition, 2006. 55

[114] S. Losse, J. G. Vos, and S. Rau. Catalytic hydrogen production at cobalt centres. $\mathrm{Co}$ ord. Chem. Rev., 254(21-22):2492-2504, 2010. ISSN 0010-8545. doi: 10.1016/j.ccr.2010.06.004. URL http://www.sciencedirect.com/science/ article/pii/S001085451000158X 19 41

[115] P. Lustenberger, H. Rohrer, R. Christoph, and H. Siegenthaler. Scanning tunneling microscopy at potential controlled electrode surfaces in electrolytic environment. J. Electroanal. Chem., 243(1):225-235, March 1988. ISSN 0022-0728. doi: 10.1016/0022-0728(88)85043-5. URL http://www.sciencedirect.com/science/ article/pii/0022072888850435[5]29] 


\section{REFERENCES}

[116] J. V. Macpherson and P. R. Unwin. Determination of the Diffusion Coefficient of Hydrogen in Aqueous Solution Using Single and Double Potential Step Chronoamperometry at a Disk Ultramicroelectrode. Anal. Chem., 69(11):2063-2069, 1997. doi: 10.1021/ac961211i. URL http:// pubs.acs.org/doi/abs/10.1021/ac961211i 20

[117] O. M. Magnussen. Ordered Anion Adlayers on Metal Electrode Surfaces. Chem. Rev., 102(3): 679-726, 2002. URL http://pubs.acs.org/doi/ abs/10.1021/cr000069p 15, 16 [3]

[118] O. M. Magnussen, J. Hotlos, R. J. Nichols, D. M. Kolb, and R. J. Behm. Atomic structure of $\mathrm{Cu}$ adlayers on $\mathrm{Au}(100)$ and $\mathrm{Au}(111)$ electrodes observed by in situ scanning tunneling microscopy. Phys. Rev. Lett., 64:29292932, Jun 1990. doi: 10.1103/PhysRevLett.64. 2929. URL http://link.aps.org/doi/10.1103/ PhysRevLett.64.2929.25

[119] O. M. Magnussen, J. Hotlos, G. Beitel, D. M. Kolb, and R. J. Behm. Atomic structure of ordered copper adlayers on single-crystalline gold electrodes. J. Vac. Sci. Technol., B, 9(2):969975, March 1991. URL http://link.aip.org/ link/?JVB/9/969/1 12

[120] O. M. Magnussen, J. Hageb?ck, J. Hotlos, and R. J. Behm. In situ scanning tunnelling microscopy observations of a disorderorder phase transition in hydrogensulfate adlayers on $\mathrm{Au}(111)$. Faraday Discuss., 94:329338, 1992. URL http://dx.doi.org/10.1039/ FD9929400329 2, $15,16,33$

[121] N. Marsot, R. Belkhou, F. Scheurer, B. Bartenlian, N. Barrett, M. Delaunay, and C. Guillot. Characterization of the $\mathrm{Co} / \mathrm{Au}(111)$ interface by core-level photoemission electron spectroscopy. Surf. Sci., 377379(0):225-228, 1997. ISSN 0039-6028. doi: 10.1016/S0039-6028(96) 01360-X. URL http://www.sciencedirect.com/ science/article/pii/S003960289601360X 39 41

[122] J. Matsushima, F. Trivinho-Strixino, and E. Pereira. Investigation of cobalt deposition using the electrochemical quartz crystal microbalance. Electrochim. Acta, 51(10):1960-1966, 2006. ISSN 0013-4686. doi: 10.1016/j.electacta.2005. 07.003. URL http://www.sciencedirect.com/ science/article/pii/S0013468605007097 [17 21

[123] J. C. Maxwell. A Dynamical Theory of the Electromagnetic Field. Philosophical Transactions of the Royal Society of London, 155: 459-512, 1865. doi: 10.1098/rstl.1865.0008. URL http://rstl.royalsocietypublishing. org/content/155/459.short [1]
[124] A. J. Melmed. The art and science and other aspects of making sharp tips. Fifth international conference on scanning tunneling microscopy/spectroscopy, 9(2):601-608, 1991. doi: 10.1116/1.585467. URL http://link.aip.org/ link/?JVB/9/601/1 31

[125] L. Mendoza-Huizar, J. Robles, and M. PalomarPardavé. Nucleation and growth of cobalt onto different substrates: Part II. The updopd transition onto a gold electrode. J. Electroanal. Chem., 545(0):39-45, 2003. ISSN 15726657. doi: 10.1016/S0022-0728(03)00087-1. URL http://www.sciencedirect.com/science/ article/pii/S0022072803000871 [21]

[126] H. B. Michaelson. The work function of the elements and its periodicity. J. Appl. Phys., 48(11): 4729-4733, 1977. doi: 10.1063/1.323539. URL http://link.aip.org/link/?JAP/48/4729/1 30

[127] T. Miyazaki and N. Tezuka. Giant magnetic tunneling effect in $\mathrm{Fe} / \mathrm{Al} 2 \mathrm{O} 3 / \mathrm{Fe}$ junction. J. Magn. Magn. Mater., 139(3):L231-L234, 1995. ISSN 0304-8853. doi: 10.1016/0304-8853(95)90001-2. URL http://www.sciencedirect.com/science/ article/pii/0304885395900012 46

[128] J. S. Moodera and G. Mathon. Spin polarized tunneling in ferromagnetic junctions. J. Magn. Magn. Mater., 200(1-3):248-273, 1999. ISSN 0304-8853. doi: 10.1016/S0304-8853(99)00515-6. URL http://www.sciencedirect.com/science/ article/pii/S0304885399005156 47

[129] K. Morgenstern, J. Kibsgaard, J. V. Lauritsen, E. Lgsgaard, and F. Besenbacher. Cobalt growth on two related close-packed noble metal surfaces. Surf. Sci., 601(9):1967-1972, 2007. ISSN 0039-6028. doi: 10.1016/j.susc.2007.02.018. URL http://www.sciencedirect.com/science/ article/pii/S0039602807001446 [35] 36 [39

[130] K. Mukasa, K. Sueoka, H. Hasegawa, Y. Tazuke, and K. Hayakawa. Spin-polarized STM and its family. Mater. Sci. Eng., B, 31(1-2):69-76, April 1995. ISSN 09215107. URL http://www.sciencedirect. com/science/article/B6TXF-404RNX5-11/2/ f4eeab0eb4da4baeb0976ea6f10ec83a. 45

[131] L. A. Nagahara, T. Thundat, and S. M. Lindsay. Preparation and characterization of STM tips for electrochemical studies. Rev. Sci. Instrum., 60(10):3128-3130, 1989. doi: 10.1063/ 1.1140590. URL http://link.aip.org/link/ ?RSI/60/3128/1 33

[132] G. Nagy. Water structure at the graphite(0001) surface by STM measurements. J. Electroanal. Chem., 409(12):19-23, 1996. ISSN 1572-6657. doi: 10.1016/0022-0728(95)04409-4. 
URL http://www.sciencedirect.com/science/ article/pii/0022072895044094. 30

[133] G. Nagy and T. Wandlowski. Double Layer Properties of $\mathrm{Au}(111) / \mathrm{H}_{2} \mathrm{SO}_{4}(\mathrm{Cl})+\mathrm{Cu}^{2+}$ from Distance Tunneling Spectroscopy. Langmuir, 19(24):10271-10280, 2003. doi: 10.1021/ la034950k. URL http://pubs.acs.org/doi/abs/ 10.1021/la034950k 30

[134] W. H. Nernst. Die elektromotorische Wirksamkeit der Ionen. Zeitschrift für physikalische Chemie, Stöchiometrie und Verwandtschaftslehre, 4:129-181, 1889. URL http://electrochem.cwru.edu/estir/hist/ hist-13-Nernst-1.pdf 7

[135] H. P. Oepen, Y. T. Millev, and J. Kirschner. The reorientation transition in $\mathrm{Co} / \mathrm{Au}(111)$. J. Appl. Phys., 81(8):5044-5046, 1997. doi: 10.1063/1. 364502. URL http://link .aip.org/link/?JAP/ 81/5044/1 71

[136] Oersted. Ein electrisch-magnetischer versuch. Annalen der Physik, 73(3):278-278, 1823. ISSN 1521-3889. doi: 10.1002/andp. 18230730307. URL http://dx.doi.org/10. 1002/andp.18230730307. 1

[137] I. Ohnaka. Melt Spinning into a Liquid Cooling Medium. Int. J. Rapid Solidif., 1:219-236, 19841985. 3153

[138] S. Ohnuma and T. Masumoto. Zero magnetostriction and low field magnetic properties of $\mathrm{Co}-\mathrm{Fe}$ base amorphous alloys containing $\mathrm{V}, \mathrm{Nb}, \mathrm{Ta}, \mathrm{Cr}, \mathrm{Mo}, \mathrm{W}, \mathrm{Mn}$ or Ni. J. Appl. Phys., 50(B11):7597-7599, 1979. doi: 10.1063/1. 326856. URL http://link . aip.org/link/?JAP/ 50/7597/1 54

[139] S. Padovani, P. Molinàs-Mata, F. Scheurer, and J. Bucher. Variable temperature STM and Kerr studies of ultrathin films of $\mathrm{Co}$ on $\mathrm{Au}(111)$ : from self-organized clusters to continuous films. Appl. Phys. A, 66:1199-1203, 1998. ISSN 0947-8396. URL http://dx.doi.org/10.1007/ s003390051326 10.1007/s003390051326. 36 [71

[140] S. Padovani, I. Chado, F. Scheurer, and J. P. Bucher. Transition from zero-dimensional superparamagnetism to two-dimensional ferromagnetism of Co clusters on $\mathrm{Au}(111)$. Phys. Rev. B, 59(18):11887-11891, May 1999. URL http://link.aps.org/doi/10.1103/PhysRevB. 59.11887, 36 72

[141] M. Palomar-Pardavé, B. Scharifker, E. Arce, and M. Romero-Romo. Nucleation and diffusioncontrolled growth of electroactive centers: Reduction of protons during cobalt electrodeposition. Electrochim. Acta, 50(24):4736-4745, 2005.
ISSN 0013-4686. doi: 10.1016/j.electacta.2005. 03.004. URL http://www.sciencedirect.com/ science/article/pii/S0013468605002045 [19 41

[142] J. Pan, T. W. Jing, and S. M. Lindsay. Tunneling Barriers in Electrochemical Scanning Tunneling Microscopy. J. Phys. Chem., 98(16):4205-4208, 1994. doi: 10.1021/ j100067a001. URL http://pubs.acs.org/doi/ abs/10.1021/j100067a001]30

[143] J. Perez, E. R. Gonzalez, and H. M. Villullas. Hydrogen Evolution Reaction on Gold SingleCrystal Electrodes in Acid Solutions. J. Phys. Chem. B, 102(52):10931-10935, December 1998. ISSN 1520-6106. URL http://dx.doi.org/10. 1021/jp9831987. PH: 170. 13]

[144] A. R. Piercy. The changing shape of magnetostriction. Phys. Educ., 32(3):160-160, 1997. URL http://stacks.iop.org/0031-9120/32/i= $3 / \mathrm{a}=014,53,54,56$

[145] N. V. Plyasunova, Y. Zhang, and M. Muhammed. Critical evaluation of thermodynamics of complex formation of metal ions in aqueous solutions. $\mathrm{V}$. hydrolysis and hydroxo-complexes of $\mathrm{Co}^{2+}$ at 298.15 K. Hydrometallurgy, 48(2):153-169, 1998. ISSN 0304-386X. doi: 10.1016/S0304-386X(97) 00078-9. URL http://www.sciencedirect.com/ science/article/pii/S0304386X97000789, 21]

[146] K. M. Poduska and S. Morin. Electrochemical cell for in situ magneto-optic Kerr effect measurements. Rev. Sci. Instrum., 74(11):4723-4727, November 2003. URL http://dx.doi.org/10. 1063/1.1619583, $1,45,72$

[147] D. Powell, J. Cortez, and E. K. Mellon. A laboratory exercise introducing students to the Pourbaix for cobalt. Journal of Chemical Education, 64(2):165, 1987 . doi: 10.1021/ ed064p165. URL http://pubs.acs.org/doi/ abs/10.1021/ed064p165 17

[148] M. G. D. Ppolo, E. P. M. Leiva, M. Mariscal, and W. Schmickler. The basis for the formation of stable metal clusters on an electrode surface. Nanotechnology, 14(9):1009-1013, 2003. URL http://stacks.iop.org/0957-4484/14/1009. 39

[149] P. Prod'homme, F. Maroun, R. Corts, P. Allongue, J. Hamrle, J. Ferr, J.-P. Jamet, and N. Vernier. Preparation, characterization and magneto-optical investigations of electrodeposited $\mathrm{Co} / \mathrm{Au}$ films. J. Magn. Magn. Mater., 315(1):26-38, August 2007. ISSN 0304-8853. URL http://www.sciencedirect. com/science/article/B6TJJ-4N6NK1M-2/2/ c6b3e17e5df1956f6364ec80907d9954 72 


\section{REFERENCES}

[150] S. Pütter, H. F. Ding, Y. T. Millev, H. P. Oepen, and J. Kirschner. Magnetic susceptibility: An easy approach to the spin-reorientation transition. Phys. Rev. B, 64(9):092409, August 2001. URL http://link. aps.org/doi/10.1103/ PhysRevB.64.092409 36. 71]

[151] M. V. Rastei and J. P. Bucher. Spin polarized tunnelling investigation of nanometre Co clusters by means of a Ni bulk tip. J. Phys.: Condens. Matter, 18(50):L619-L624, 2006. URL http://stacks.iop.org/0953-8984/18/L619 45

[152] V. Repain, J. Berroir, S. Rousset, and J. Lecoeur. Growth of self-organized cobalt nanostructures on $\mathrm{Au}(111)$ vicinal surfaces. Surf. Sci., 447(13):L152-L156, 2000. ISSN 00396028. doi: 10.1016/S0039-6028(99)01203-0. URL http://www.sciencedirect.com/science/ article/pii/S0039602899012030 36 38

[153] G. Repphun and J. Halbritter. Tunnel channels, charge transfer, and imaging mechanisms in scanning tunneling microscopy. J. Vac. Sci. Technol., A, 13(3):1693-1698, May 1995. URL http:// link.aip.org/link/?JVA/13/1693/1. PH: 166. 30

[154] E. Ruska. The emergence of the electron microscope: Connection between Realization and First Patent Application, Documents of an Invention. J. Ultrastruct. Mol. Struct. Res., 95(1-3):3-28, April 1986. ISSN 0889-1605. URL http://www.sciencedirect.com/science/ article/pii/088916058690025X [2

[155] E. Ruska. Das Entstehen des Elektronenmikroskops und der Elektronenmikroskopie (Nobel-Vortrag). Angew. Chem., 99(7):611621 1987. ISSN 1521-3757. doi: 10.1002/ ange.19870990704. URL http://dx.doi.org/10. 1002/ange.19870990704 2

[156] E. Ruska. The Development of the Electron Microscope and of Electron Microscopy (Nobel Lecture). Angew. Chem., Int. Ed., 26(7):595605, 1987. ISSN 1521-3773. doi: 10.1002/anie. 198705953. URL http://dx.doi.org/10.1002/ anie.198705953 2

[157] S. Saito, K. Takeda, T. Soumura, M. Ohki, T. Tani, and T. Maeda. Hysteresis of the work function of $\mathrm{Co}(0001)$ surface resulting from an allotropic transformation. J. Appl. Phys., 71(11): 5500-5503, 1992. doi: 10.1063/1.350523. URL http://link.aip.org/link/?JAP/71/5500/1 30

[158] S. Saito, K. Takeda, T. Soumura, T. Tani, and T. Maeda. Effects of surface roughness and patches on the work function of cobalt. Phys. Status Solidi A, 142(1):K29-K32, 1994. ISSN 1521-396X. doi: 10.1002/pssa.
2211420140. URL http://dx.doi.org/10.1002/ pssa.2211420140 30

[159] J. Sass and J. Gimzewski. Solvent dynamical effects in scanning tunneling microscopy with a polar liquid in the gap. J. Electroanal. Chem., 308(1-2):333-337, June 1991. ISSN 0022-0728. URL http://www.sciencedirect. com/science/article/B6TGB-44X7511-5S/2/ eb04f765e83140b8e0ecc3a5227ca92a PH: 167. 30

[160] W. Schindler and J. Kirschner. New electrochemical cell for in situ tunneling microscopy, cyclovoltammetry, and optical measurements. Rev. Sci. Instrum., 67(10):3578-3582, October 1996. URL http://link.aip.org/link/?RSI/ 67/3578/1 511

[161] W. Schindler and J. Kirschner. Ultrathin magnetic films: Electrochemistry versus molecularbeam epitaxy. Phys. Rev. B, 55(4):R1989-R1992, January 1997. URL http://link.aps.org/doi/ 10.1103/PhysRevB.55.R1989, 1, 5 , 45,72

[162] W. Schindler, T. Koop, D. Hofmann, and J. Kirschner. Reversible electrodeposition of ultrathin magnetic Co films. IEEE T. Magn., 34 (4):963-967, 1998. ISSN 0018-9464. doi: 10. 1109/20.706327. 1 , $5,45,72$

[163] W. Schindler, T. Koop, and J. Kirschner. Growth and magnetism of ultrathin electrodeposited Co films. Z. Physik. Chem., 208(1-2):93-106, 1999. doi: 10.1524/zpch.1999.208.Part_1_2.093. URL http://www.oldenbourg-link.com/doi/abs/10. 1524/zpch.1999.208.Part_1_2.093]1]

[164] W. Schindler, D. Hofmann, and J. Kirschner. Localized Electrodeposition Using a Scanning Tunneling Microscope Tip as a Nanoelectrode. $J$. Electrochem. Soc., 148(2):C124-C130, 2001. doi: 10.1149/1.1343107. URL http://link.aip.org/ link/?JES/148/C124/1 233

[165] W. Schindler, P. Hugelmann, M. Hugelmann, and F. Kärtner. Localized electrochemical nucleation and growth of low-dimensional metal structures. J. Electroanal. Chem., 522(1):49-57, March 2002. ISSN 15726657. URL http://www.sciencedirect. com/science/article/B6TGB-44RNJHV-5/2/ b2213d748be132f $46 \mathrm{ccdb} 1 \mathrm{e} 2 \mathrm{c} 32 \mathrm{~d} 680 \mathrm{a}$. 2

[166] W. Schindler, M. Hugelmann, and P. Hugelmann. In situ scanning probe spectroscopy at nanoscale solid/liquid interfaces. Electrochim. Acta, 50(15):3077-3083, May 2005. ISSN 0013-4686. URL http://www.sciencedirect. com/science/article/B6TG0-4G1R3YP-3/2/ 48ebace8f9986a4984c36af4bb38f636. 2] 
[167] W. Schindler and P. Hugelmann. Localized Electrocrystallization of Metals by STM Tip Nanoelectrodes. In D. G. Staikov, editor, Electrocrystallization in Nanotechnology, pages 115137. John Wiley and Sons, 2007. URL http: //dx.doi.org/10.1002/9783527610198.ch6 [2

[168] U. Schlickum, W. Wulfhekel, and J. Kirschner. Spin-polarized scanning tunneling microscope for imaging the in-plane magnetization. Appl. Phys. Lett., 83(10):2016-2018, 2003. doi: 10.1063/ 1.1606867. URL http://link.aip.org/link/ ?APL/83/2016/1. 4969

[169] W. Schmickler and D. Henderson. A model for the scanning tunneling microscope operating in an electrolyte solution. J. Electroanal. Chem., 290(12):283-291, 1990. ISSN 0022-0728. doi: 10.1016/0022-0728(90)87439-Q. URL http://wWw.sciencedirect.com/science/ article/pii/002207289087439Q 30

[170] W. Schmickler. Tunneling of electrons through thin layers of water. Surf. Sci., $335(0): 416-421, \quad 1995 . \quad$ ISSN $0039-6028$. doi: 10.1016/0039-6028(95)00451-3. URL http://www.sciencedirect.com/science/ article/pii/0039602895004513 30

[171] W. Schmickler. Grundlagen der Elektrochemie. Fried. Vieweg \& Sohn Verlagsgesellschaft mbH, Braunschweig/Wiesbaden, 1996. URL http://www.springer.com/chemistry/ physical+chemistry/book/978-3-540-67045-2.

[172] K. Schouteden, K. Lauwaet, D. A. Muzychenko, P. Lievens, and C. V. Haesendonck. Spindependent electronic structure of self-organized Co nanomagnets. New J. Phys., 13(3):033030, 2011. URL http://stacks .iop.org/1367-2630/ $13 / i=3 / a=03303045$

[173] I. Sebastian, M. Heiler, K. Meinel, and H. Neddermeyer. Growth of epitaxial layers of Co and $\mathrm{CoO}$ on $\mathrm{Au}(111)$. Appl. Phys. A, 66:525-528, 1998. ISSN 0947-8396. URL http://dx.doi. org/10.1007/s003390051195 36

[174] R. Sellmann, H. Fritzsche, and H. Maletta. Growth of thin Co films on W(110) and $\mathrm{Au}(111)$ layers prepared on $\mathrm{Al} 2 \mathrm{O} 3(11 \overline{2} 0)$. Surf. Sci., 495(1-2):185-194, 2001. ISSN 00396028. doi: 10.1016/S0039-6028(01)01569-2. URL http://www.sciencedirect.com/science/ article/pii/S0039602801015692 35

[175] J. C. Slonczewski. Conductance and exchange coupling of two ferromagnets separated by a tunneling barrier. Phys. Rev. B, 39(10):6995-7002, April 1989. doi: 10.1103/PhysRevB.39.6995. URL http://link.aps.org/abstract/PRB/v39/ p6995 4647
[176] M.-B. Song, J.-M. Jang, S.-E. Bae, and C.W. Lee. Charge Transfer through Thin Layers of Water Investigated by STM, AFM, and QCM. Langmuir, 18(7):2780-2784, 2002. doi: 10.1021/la011189a. URL http://pubs .acs.org/ doi/abs/10.1021/la011189a 30

[177] R. Sonnenfeld and P. K. Hansma. AtomicResolution Microscopy in Water. Science, 232 (4747):211-213, 1986. doi: 10.1126/science.232. 4747.211. URL http://www.sciencemag.org/ content/232/4747/211. abstract $5[29$

[178] R. Sonnenfeld, J. Schneir, B. Drake, P. K. Hansma, and D. E. Aspnes. Semiconductor topography in aqueous environments: Tunneling microscopy of chemomechanically polished (001) GaAs. Appl. Phys. Lett., 50(24):1742-1744, 1987. doi: 10.1063/1.97734. URL http://link.aip. org/link/?APL/50/1742/1 29

[179] B. Speiser. Elektroanalytische Methoden I: Elektrodenreaktionen und Chronoamperometrie. Chem. Unserer Zeit, 15(1):21-26, 1981. ISSN 1521-3781. doi: 10.1002/ciuz. 19810150105. URL http://dx.doi.org/10. 1002/ciuz.19810150105 2

[180] B. Speiser. Elektroanalytische Methoden II: Cyclische Voltammetrie. Chem. Unserer Zeit, 15 (2):62-67, 1981. ISSN 1521-3781. doi: 10.1002/ ciuz.19810150206. URL http://dx.doi.org/10. 1002/ciuz.19810150206 11

[181] M. Stecher. The Moiré Phenomenon. Am. J. Phys., 32(4):247-257, 1964 . doi: 10.1119/ 1.1970239. URL http://link.aip.org/link/ ?AJP/32/247/1 35

[182] O. Stern. Zur theorie der elektrolytischen doppelschicht. Zeitschrift für Elektrochemie und angewandte physikalische Chemie, 30:508516, 1924. URL http://electrochem.cwru.edu/ estir/hist/hist-81-0Stern.pdf 8

[183] U. Stimming, R. Vogel, D. Kolb, and T. Will. Metal deposition and dissolution monitored by In situ scanning tunneling microscopy. $J$. Power Sources, 43(13):169-180, $1993 . \quad$ ISSN 0378-7753. doi: 10.1016/0378-7753(93)80113-4. URL http://www.sciencedirect.com/science/ article/pii/0378775393801134 [35]

[184] J. Stöhr. Exploring the microscopic origin of magnetic anisotropies with X-ray magnetic circular dichroism (XMCD) spectroscopy. J. Magn. Magn. Mater., 200(13):470-497, 1999. ISSN 0304-8853. doi: 10.1016/S0304-8853(99)00407-2. URL http://www.sciencedirect.com/science/ article/pii/S0304885399004072 1 [71 


\section{REFERENCES}

[185] J. Stöhr and H. C. Siegmann. Magnetism, From Fundamentals to Nanoscale Dynamics. Number 152 in Solid-State Science. Springer-Verlag Berlin Heidel, 2006. 1, 47,71

[186] V. Sudha and M. Sangaranarayanan. Underpotential deposition of metals Progress and prospects in modelling. J. Chem. Sci., 117: 207-218, 2005. ISSN 0253-4134. doi: 10. 1007/BF02709289. URL http://dx.doi.org/ 10.1007/BF02709289 8

[187] H. Takeshita, Y. Suzuki, H. Akinaga, W. Mizutani, K. Ando, T. Katayama, A. Itoh, and K. Tanaka. Magnetization process of a nanometer-scale cobalt dots array formed on a reconstructed $\mathrm{Au}(111)$ surface. J. Magn. Magn. Mater., 165(13):38-41, 1997. ISSN 03048853. doi: 10.1016/S0304-8853(96)00468-4. URL http://www.sciencedirect.com/science/ article/pii/S0304885396004684. 72

[188] J. Tersoff. Role of tip electronic structure in scanning tunneling microscope images. Phys. Rev. B, 41(2):1235-1238, January 1990. URL http://link.aps.org/doi/10.1103/ PhysRevB.41.1235 26]

[189] J. Tersoff and D. R. Hamann. Theory and Application for the Scanning Tunneling Microscope. Phys. Rev. Lett., 50(25):1998-2001, June 1983. URL http://link.aps.org/doi/10.1103/ PhysRevLett.50.1998 $25 \mid 26$

[190] J. Tersoff and D. R. Hamann. Theory of the scanning tunneling microscope. Phys. Rev. B, 31: 805-813, Jan 1985. doi: 10.1103/PhysRevB.31. 805. URL http://link.aps.org/doi/10.1103/ PhysRevB.31.805. 25].26

[191] C. Tölkes, P. Zeppenfeld, M. A. Krzyzowski, R. David, and G. Comsa. Growth and stability of cobalt nanostructures on gold (111). Surf. Sci., 394(13):170-184, 1997. ISSN 00396028. doi: 10.1016/S0039-6028(97)00605-5. URL http://www.sciencedirect.com/science/ article/pii/S0039602897006055, 36 41

[192] J. Unguris, M. R. Scheinfein, R. J. Celotta, and D. T. Pierce. Magnetic microstructure of the (0001) surface of hcp cobalt. Appl. Phys. Lett., 55(24):2553-2555, 1989. doi: 10.1063/1. 101979. URL http://link.aip.org/link/?APL/ 55/2553/1 71

[193] A. Vaught, T. Jing, and S. Lindsay. Nonexponential tunneling in water near an electrode. Chem. Phys. Lett., 236(3):306-310, 1995. ISSN 0009-2614. doi: 10.1016/0009-2614(95)00223-Q. URL http://www.sciencedirect.com/science/ article/pii/000926149500223Q 30
[194] B. Voigtländer, G. Meyer, and N. M. Amer. Epitaxial growth of thin magnetic cobalt films on $\mathrm{Au}(111)$ studied by scanning tunneling microscopy. Phys. Rev. B, 44(18):10354-10357, November 1991. URL http://link.aps.org/

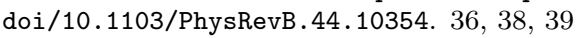

[195] D. Weller, J. Stöhr, R. Nakajima, A. Carl, M. G. Samant, C. Chappert, R. Mégy, P. Beauvillain, P. Veillet, and G. A. Held. Microscopic Origin of Magnetic Anisotropy in $\mathrm{Au} / \mathrm{Co} / \mathrm{Au}$ Probed with X-Ray Magnetic Circular Dichroism. Phys. Rev. Lett., 75:3752-3755, Nov 1995. doi: 10.1103/ PhysRevLett.75.3752. URL http://link.aps . org/doi/10.1103/PhysRevLett.75.3752 77172

[196] J. Wiechers, T. Twomey, D. Kolb, and R. Behm. An in-situ scanning tunneling microscopy study of $\mathrm{Au}(111)$ with atomic scale resolution. $J$. Electroanal. Chem., 248(2):451-460, 1988. ISSN 0022-0728. doi: 10.1016/0022-0728(88)85106-4. URL http://www.sciencedirect.com/science/ article/pii/0022072888851064, 5, 29

[197] R. Wiesendanger. Scanning Probe Microscopy and Spectroscopy: Methods and Applications. Cambridge University Press, 1994. URL http: //dx.doi.org/10.1017/CB09780511524356 25]

[198] R. Wiesendanger, H.-J. Güntherodt, G. Güntherodt, R. J. Gambino, and R. Ruf. Observation of vacuum tunneling of spinpolarized electrons with the scanning tunneling microscope. Phys. Rev. Lett., 65(2):247-250, Jul 1990. doi: 10.1103/PhysRevLett.65.247. URL http://link.aps.org/doi/10.1103/ PhysRevLett.65.247. 45, 49

[199] Wiesendanger, R. Spin mapping at the nanoscale and atomic scale. Rev. Mod. Phys., 81(4): 1495-1550, November 2009. doi: 10.1103/ RevModPhys.81.1495. URL http://link.aps. org/abstract/RMP/v81/p1495 $2,45,48,49$

[200] D. Wortmann, S. Heinze, P. Kurz, G. Bihlmayer, and S. Blügel. Resolving Complex Atomic-Scale Spin Structures by Spin-Polarized Scanning Tunneling Microscopy. Phys. Rev. Lett., 86(18):41324135, April 2001. URL http://link.aps.org/ doi/10.1103/PhysRevLett.86.4132 48

[201] W. Wulfhekel. High Resolution Magnetic Imaging by Local Tunneling Magnetoresistance. In H. Hopster and H. P. Oepen, editors, Magnetic Microscopy of Nanostructures, chapter 9, pages 181-202. Springer Berlin Heidelberg, 2005. doi: 10.1007/3-540-26641-0_9. 85

[202] W. Wulfhekel and J. Kirschner. Spin-polarized scanning tunneling microscopy on ferromagnets. Appl. Phys. Lett., 75(13):1944-1946, September 1999. URL http://link.aip.org/link/?APL/ 75/1944/1 III IV] 3 [5, 49 69 
[203] W. Wulfhekel and J. Kirschner. Spin-Polarized Scanning Tunneling Microscopy of Magnetic Structures and Antiferromagnetic Thin Films. Annu. Rev. Mater. Res., 37(1):6991, August 2007. ISSN 1531-7331. URL http://dx.doi.org/10.1146/annurev.matsci. $37.052506 .084342[269$

[204] W. Wulfhekel, H. Ding, W. Lutzke, G. Steierl, M. Vázquez, P. Marín, A. Hernando, and J. Kirschner. High-resolution magnetic imaging by local tunneling magnetoresistance. Appl. Phys. A, 72(4): 463-470, 2001. ISSN 0947-8396. URL http://dx.doi.org/10.1007/s003390100579 78

[205] W. Wulfhekel, R. Hertel, H. F. Ding, G. Steierl, and J. Kirschner. Amorphous, low magnetostriction tips for spin-polarized scanning tunneling microscopy. J. Magn. Magn. Mater., 249(1-2):368-374, August 2002. ISSN 03048853. URL http://www.sciencedirect. com/science/article/B6TJJ-46HG22S-6/2/ b244159e9ffb121e5c63214eaa1a0339 60

[206] J. Xu, M. A. Howson, B. J. Hickey, D. Greig, E. Kolb, P. Veillet, and N. Wiser. Superparamagnetism and different growth mechanisms of $\mathrm{Co} / \mathrm{Au}(111)$ and $\mathrm{Co} / \mathrm{Cu}(111)$ multilayers grown by molecular-beam epitaxy. Phys. Rev. B, 55: 416-422, Jan 1997. doi: 10.1103/PhysRevB.55. 416. URL http://link.aps.org/doi/10.1103/ PhysRevB.55.416 72

[207] R. Young, J. Ward, and F. Scire. Observation of Metal-Vacuum-Metal Tunneling, Field Emission, and the Transition Region. Phys. Rev. Lett., 27 (14):922-924, October 1971. URL http://link. aps.org/doi/10.1103/PhysRevLett.27.922 [30

[208] J. Zhang and J. Ulstrup. Oxygen-free in situ scanning tunnelling microscopy. J. Electroanal. Chem., 599(2):213-220, 2007. ISSN 1572-6657. doi: 10.1016/j.jelechem.2006.02.011. URL http://www.sciencedirect.com/science/ article/pii/S0022072806000787 Special Issue In Honour of David Schiffrin. 14

[209] J. Ziegler, R. Wielgosz, and D. Kolb. Pb deposition on $\mathrm{n}-\mathrm{Si}(111)$ electrodes. Electrochim. Acta, 45(45):827-833, 1999. ISSN 0013-4686. doi: 10.1016/S0013-4686(99)00261-3. URL http://www.sciencedirect.com/science/ article/pii/S0013468699002613 35

[210] C. G. Zoski. Handbook of Electrochemistry. Elsevier Science \& Technology, 2007. URL http: //lib.myilibrary.com?ID=75178 $16,17,20$ 
REFERENCES 


\section{Acknowledgments}

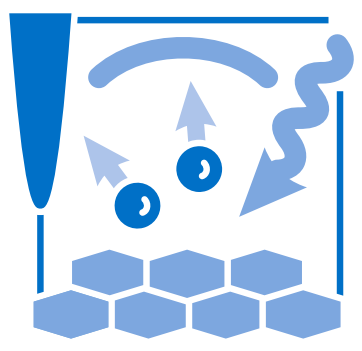

To this work presented here a lot of people contributed directly or indirectly and I would like to thank everybody making this thesis possible. It is not that easy to find the right words since this is the last page and shouldn't be one more chapter. I would like to give my sincere thanks to

- Prof. Dr. Johannes V. Barth for giving my the opportunity to do my thesis at E20. It was great a time being part of this work group.

- PD Dr. Werner Schindler for supervising my and giving me the opportunity to work in this great field of physics.

- Dr. Matthias Opel at the Walther-Meißner-Institut for the opportunity and his friendly support to do the SQUID measurements presented here.

- Prof. Dr. Peter Feulner for the fruitful discussions and his help whenever I was asking.

- Mr. Ken-ichi Bushida at UNITIKA for the AC20 wire he sent me from Japan.

- all the members of the electronics workshop as well as of the crystal and material laboratory for their support in preparation of coils and tips.

- the working group E10 for the temporary provisioning of purified water and support at the SEM.

- Karl Kölbl, Reinhold Schneider, Max Glanz, Viktoria Blaschek, Karl Eberle, and Kamila Wilson for supporting me in technical questions and fights against paperwork.

- Knud Seufert, Matthias Marschall, Daniel Gerster, Wolfgang Krenner, Dirk Kühne, Sebastian Jakob, Richard Steinacher, Felix Bischoff, and Alissa Wiengarten, as well as our visitors Florian Blobner and Tobias Kaposi - the best 
office in town and friends. You all created this great environment for working and having fun at the same time.

- Wolfgang Krenner, Tobias Kaposi, Anthoula Papageorgiou, and Florian Blobner for their great support.

- Katharina Diller and Sybille Fischer for all the fruitful discussions during lunch time.

- all at E20 and all the others I unintentionally forgot to name.

I would quite particularly like to thank my parents, Sybille and Klaus, for all their love and support. (Ich möchte mich an dieser Stelle bei euch beiden bedanken für eure unermüdliche Unterstützung mit Rat und Tat auch aus der Ferne, für all euer Verständins und eure Liebe. Ich hätte es nicht besser treffen können.)

Special thanks to my sister Ariane, for her wisdom and support. (Ich danke dir für deine moralische Unterstützung und Liebe. Du bist mir eine großartige Hilfe und die größte kleine Schwester, die man sich vorstellen kann.)

Last but not least, I would like to thank Tjark for being with me all the time. (Danke, dass es dich für mich und mich für dich gibt.) 

